University of Louisville

ThinkIR: The University of Louisville's Institutional Repository

8-2015

\title{
Application of surface chemistry at the interface of mesoporous TiO2 films for stable and high efficiency dye-sensitized solar cells.
}

Tulashi Luitel

University of Louisville

Follow this and additional works at: https://ir.library.louisville.edu/etd

Part of the Chemistry Commons

\section{Recommended Citation}

Luitel, Tulashi, "Application of surface chemistry at the interface of mesoporous TiO2 films for stable and high efficiency dye-sensitized solar cells." (2015). Electronic Theses and Dissertations. Paper 2252.

https://doi.org/10.18297/etd/2252

This Doctoral Dissertation is brought to you for free and open access by ThinkIR: The University of Louisville's Institutional Repository. It has been accepted for inclusion in Electronic Theses and Dissertations by an authorized administrator of ThinkIR: The University of Louisville's Institutional Repository. This title appears here courtesy of the author, who has retained all other copyrights. For more information, please contact thinkir@louisville.edu. 


\title{
APPLICATION OF SURFACE CHEMISTRY AT THE INTERFACE OF MESOPOROUS $\mathrm{TiO}_{2}$ FILMS FOR STABLE AND HIGH EFFICIENCY DYE-SENSITIZED SOLAR CELLS By
}

Tulashi Luitel

Master of Science in Physics, University of Louisville, 2008

Master of Science in Chemistry, University of Louisville, 2012

\author{
A Dissertation \\ Submitted to the Faculty of the \\ College of Arts and Sciences of the University of Louisville \\ in Partial Fulfillment of the Requirements \\ for the Degree of \\ Doctor of Philosophy \\ in Chemistry \\ Department of Chemistry \\ University of Louisville \\ Louisville, Kentucky
}

August 2015 
Copyright 2015 by Tulashi Luitel All rights reserved 



\title{
APPLICATION OF SURFACE CHEMISTRY AT THE INTERFACE OF MESOPOROUS $\mathrm{TIO}_{2}$ FILMS FOR STABLE AND HIGH EFFICIENCY DYE-SENSITIZED SOLAR CELLS
}

\author{
By \\ Tulashi Luitel \\ A Dissertation Approved on \\ July $27^{\text {th }}, 2015$ \\ by the following Dissertation Committee:
}

Dr. Francis P. Zamborini

Dissertation Director

Dr. Bruce W. Alphenaar

Dr. Richard P. Baldwin

Dr. Jinjun Liu 


\section{ACKNOWLEDGEMENTS}

This Ph.D. dissertation was developed in the Department of Chemistry at the University of Louisville and would not have been in this shape without the unconditional support and advice of department personnel and other researchers outside the department.

First of all, I would like to express my sincere acknowledgement to Professor Francis P. Zamborini for believing and giving me the opportunity to work on this fascinating project. I have tried my best to materialize his invaluable ideas and guidance in the work. I appreciate that I was provided with the autonomy of my work and decision during my Ph.D. research. I would like to extend my sincere acknowledgement to Professor Bruce W. Alphenaar, because of whom, I got expert knowledge in the measurement and the calibration of solar cells fabricated in the laboratory. I would like to express my genuine acknowledgement to Professor Richard P. Baldwin for help and encouragement not only for my Ph.D. work but also for his kindness to include me in teaching of his undergraduate laboratory classes. I am grateful to Dr. Jinjun Liu for his invaluable guidance and encouragement. This dissertation

would not have this form without his help in ultrafast laser spectroscopy. I am thankful to Tereza Paronyan for her help in teaching me how to take SEM images; also I am very grateful to Bill Richmond for his assistance in learning ATR-FTIR spectroscopy. I want to express my sincere thanks to all the members of the Department of Chemistry, Conn Center for Renewable Energy Research, Shumaker 
Research Building, Electrical Engineering and Computer science, and Department of Physics.

I am grateful to Professor Michael Grätzel for encouragement and help on how to make good electrical contact to my dye-sensitized solar cell (DSSC) devices during his 2013 visit in Louisville to receive the Leigh Ann Conn Prize. I am also grateful to Professor Carole Grätzel for suggesting to use the different sizes of semiconductor nanoparticles to improve the efficiency of DSSCs.

I cannot complete this acknowledgement without expressing my sincere thanks to the Department Chair and Professor Dr. Michael H. Nantz for his valuable suggestions about research careers. I would like to extend my sincere thanks to Professors Craig Grapperhaus for his guidance in understanding gold chemistry and Professor Marta Cecilia Yappert for her valuable help with interpreting infrared spectra. I am very grateful to the office staff of the Department of Chemistry: Sabrina Haug, Sherry Nalley, Aaron Howell, Renu Kakar, and Steve Riley. Riley's help in making fine holes on glass is appreciated. I am very grateful to former member of the office, Syble Bullock for her help in preparing travel documents. Many thanks also to all my lab mates (past and present): Olga S. Ivanova, Grzegorz W. Slawinski, Radhika Dasari, Srinivas Beeram, Monica Moreno, Rafael Masitas, Nidhi Shah, Aiqin Fang, Luther B. Hutcherson, Stacy Lynn Allen, Shekhar Bhama, Jay Narayan Sharma, Dhruba Pattadar, Pom Kharel, Brandon S. Tatum, and all the others with whom I spent very good moments. I also want to give thanks to Maria Celeste Dalfov, visiting student from Argentina (Inicio - Universidad Nacional de La Plata), who spent 3 months in our laboratory learning and understanding the basics of dye-sensitized solar cells based on $\mathrm{Ag}$ dendrimer structure. 
Last but not least, a special thanks to my family, who unconditionally supported me through the different stages of my life, wife Pramila, daughter Shreeyanka, and son Bibhus, who constantly helped me and did not lose their patience although I could not impart my time when they indeed needed me. 


\section{DEDICATION}

"Although nature commences with reason and ends in experience, it is necessary for us to do the opposite that is to commence with experience, and from this to proceed to investigate the reason."

-Leonardo da Vinci

This Dissertation is dedicated to my parents, and wife

Pandit Loknath, Radhadevi, and Pramila 


\begin{abstract}
APPLICATION OF SURFACE CHEMISTRY AT THE INTERFACE OF MESOPOROUS $\mathrm{TiO}_{2}$ FILMS FOR STABLE AND HIGH EFFICIENCY DYE-SENSITIZED SOLAR CELLS
\end{abstract}

\author{
Tulashi Luitel
}

July 27,2015

Solar energy is widely believed to be the most promising renewable energy source to fulfill the ever-increasing energy demand from human society now and into the future. Dye-sensitized solar cells (DSSCs) have been explored as a potentially low-cost alternative to silicon solar cell technology due to their lower fabrication costs compared to crystalline semiconductor photovoltaics. However, the optimized efficiency for DSSCs has not been achieved yet, and the chemical stability between dye and semiconductor has also not been addressed completely. In this research, we applied the following four main strategies to prepare photoanodes used in DSSCs with the aim of improving stability and efficiency: 1) utilizing surface chemistry to modify the porous semiconductor films, 2) linking covalently [Di-tetrabutylammoniumcis-bis (isothiocyanato) bis (2, 2'-bipyridyl-4, 4'dicarboxy-lato) ruthenium (II), N719] dye on the surface of semiconductor films, and study the charge injection dynamics by ultrafast transient absorption spectroscopy, 3) doping the semiconductor films with micro-sized neodymium oxide $\left(\mathrm{Nd}_{2} \mathrm{O}_{3}\right)$ particles, and (4) incorporating metal nanoparticles through molecular linkers to the semiconductor films. The objectives of this work are to address the limitations of chemical stability existing between $\mathrm{TiO}_{2} / \mathrm{N} 719$ dye systems, to explore the charge dynamics in $\mathrm{TiO}_{2} / \mathrm{N} 719$ system for a better 
understanding of the fundamental mechanisms of DSSCs, and to prepare high efficiency DSSCs.

We for the first time created a strong covalent amide bond between $\mathrm{TiO}_{2}$ mesoporous films and $\mathrm{N} 719$ by chemically modifying $\mathrm{TiO}_{2}$ with 3-aminopropyltrimethoxysilane. The results show the dye is air stable for more than 60 days and more resistant to UV light, thermal stress, acid, and water when compared to traditional PAs. The experiments led to another unexpected result, which was the dramatic preservation of the SCN ligand of N719 on the $\mathrm{TiO}_{2}$ surface. In most cases, there is a loss of the ligand which causes the instability of $\mathrm{N} 719$ on $\mathrm{TiO}_{2}$. This is clearly observed in the ATR-FTIR data, where the CN stretch of the SCN ligand remained present for covalently-linked dye for more than 6 months, while the $\mathrm{CN}$ stretch disappeared completely after 17-20 days from the surface of directly adsorbed N719 on $\mathrm{TiO}_{2}$. Similar results showing no degradation of the CN stretch in the ATR-FTIR data were obtained when $\mathrm{TiO}_{2}$ was chemically modified with an aromatic linker, paminophenyltrimethoxysilane (APhS), and covalently-linked with N719. The efficiency of these devices was low initially. When the charge injection dynamic at the interface of dye and $\mathrm{TiO}_{2}$ was investigated by ultrafast transient absorption spectroscopy for covalentlylinked dye, the injection rate was slower than that of traditionally-linked dye, which occurs because the linkers (APTES or APhS) increase the distance between the dye and $\mathrm{TiO}_{2}$ surfaces. However, a large amount of dye injecting very slowly was observed in the case of covalently-linked dye, which results in a higher baseline in the time region of ps. The larger baseline base clearly suggests that there were multilayers of dye on the covalently-linked dye. When these multilayers of dye were removed by dipping in acid (or water), the efficiency of the device went back to values similar to traditional photoanodes, but with an 
improved fill factor. This is an important advance in DSSC technology, allowing us to prepare more stable devices while maintaining the same efficiency.

To study the effect of plasmonic Au NPs on the surface of mesoporous $\mathrm{TiO}_{2}$, we synthesized $4 \mathrm{~nm}$ diameter Au nanoparticles (NPs) and electrostatically attached them to the mesoporous $\mathrm{TiO}_{2}$ film through APTES prior to sensitization with $\mathrm{N} 719$ dye. Results showed an overall improvement of all photoelectrochemical parameters (PEC): short-circuit current density $\left(\mathrm{J}_{\mathrm{sc}}\right)$, open circuit voltage $\left(\mathrm{V}_{\mathrm{oc}}\right)$, fill factor $(\mathrm{FF})$, and percentage efficiency $(\% \eta)$. Injection dynamics performed by UTAS clearly showed the lowest baseline in the optical density and time plot suggesting that Au facilitates the monolayer dye coverage with an increased amount of adsorbed dye and assists all dye molecules to contribute to the injection dynamics. This is in agreement with the results that both $\mathrm{J}_{\mathrm{sc}}$ and $\mathrm{V}_{\mathrm{oc}}$ were increased in these Au NP-modified photoanodes, leading to overall better PEC performance than conventional photoanodes.

We fabricated $\mathrm{TiO}_{2}-\mathrm{Nd}_{2} \mathrm{O}_{3}$ nano-micro $\left(20 \mathrm{~nm} \mathrm{TiO} 2,400 \mu \mathrm{m} \mathrm{Nd} \mathrm{N}_{3}\right.$ ) composite films to make PAs for DSSCs. This unique combination showed a 10-30\% improvement compared to traditional $\mathrm{TiO}_{2}$ films only. The Nd-doping led to a high dye-loading capacity on the photoanodes that helped to increase the short circuit current and efficiency of the devices. Electrochemical impedance spectroscopy revealed that the impedance for charge transport through the composite anode is substantially reduced compared to $\mathrm{TiO}_{2}$ alone. This decrease in resistance is possibly due to the filling of trap states in $\mathrm{TiO}_{2}$ by the $\mathrm{Nd}_{2} \mathrm{O}_{3} \mathrm{f}$ states. 
TABLE OF CONTENTS

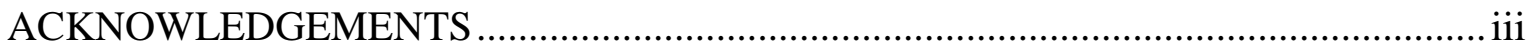

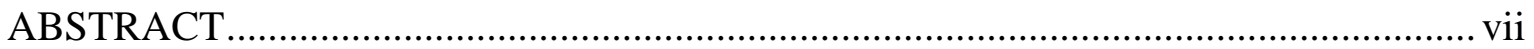

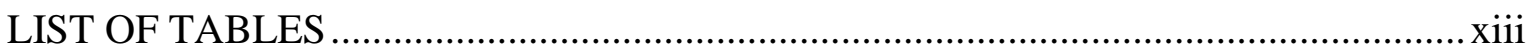

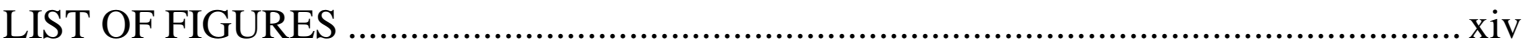

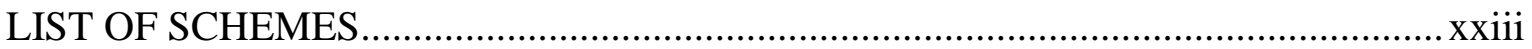

CHAPTER 1

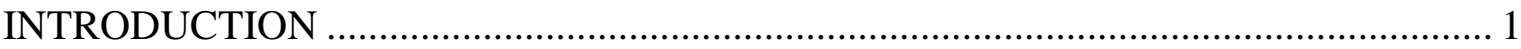

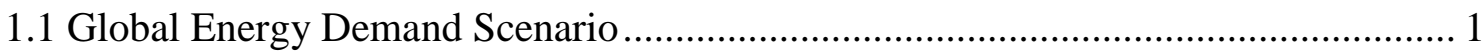

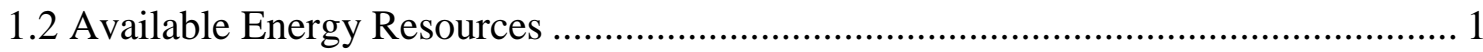

1.3 Dye-sensitized solar cells ................................................................................... 3

1.3.1 General set-up and Electrochemistry ……………........................................... 3

1.3.2 Fluorine doped tin-oxide substrate and mesoporous $\mathrm{TiO}_{2}$ semiconductor............ 8

1.3.3 Dye

1.3.4 Electrolyte ........................................................................................... 11

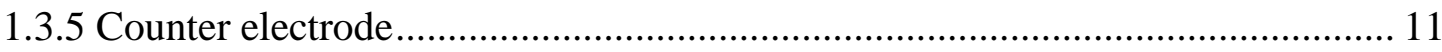

1.4 Photoelectrochemical Parameters .......................................................................... 12

1.4.1 Photocurrent Density ................................................................................ 12

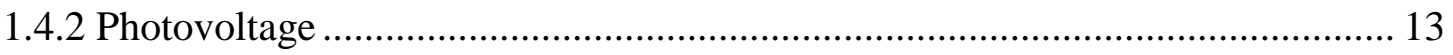

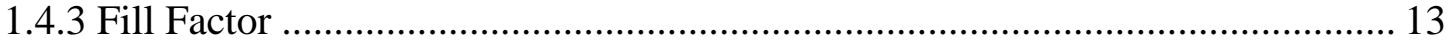

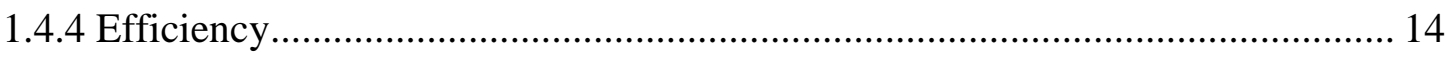

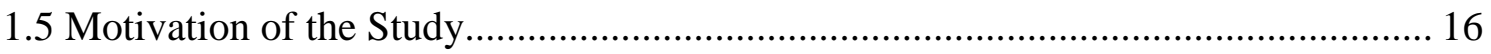

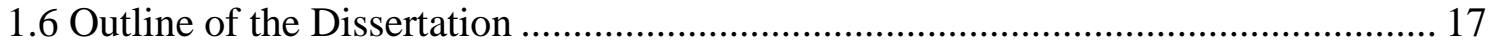

CHAPTER 2

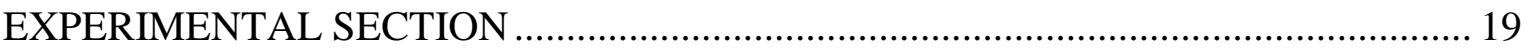

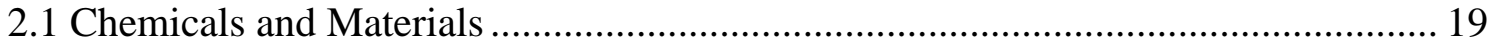


2.2 Preparation of Titanium Oxide Photoanodes ..................................................... 20

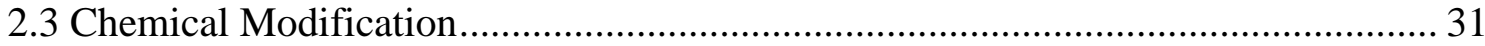

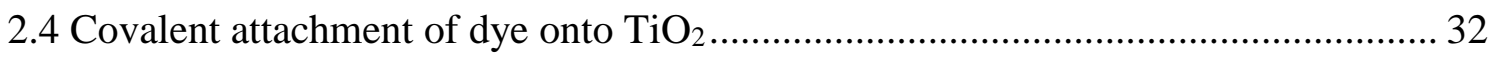

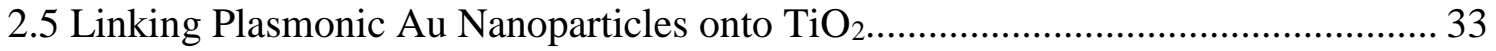

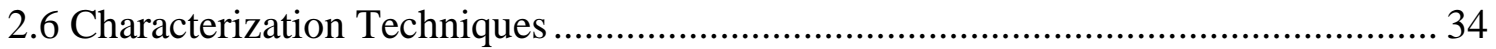

2.6.1 Steady-state UV-vis Spectroscopy (UV-vis spectroscopy) ............................. 34

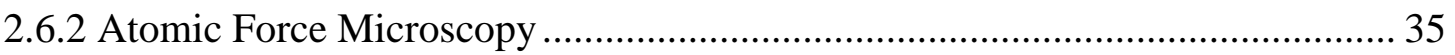

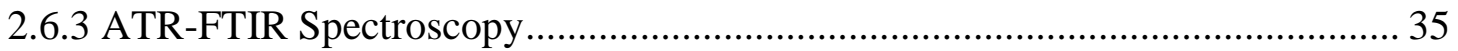

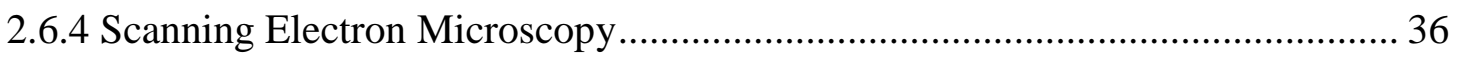

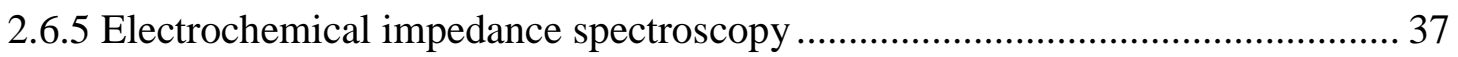

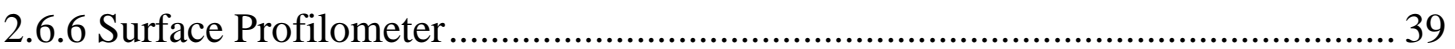

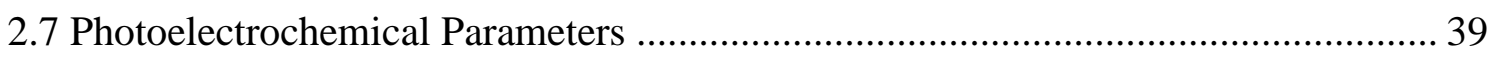

2.7.1 Photocurrent- Photovoltage Measurement ................................................... 39

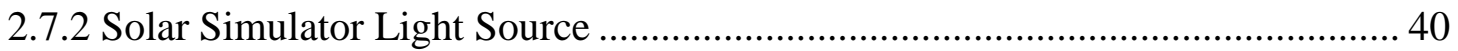

2.8 Ultrafast Transient Absorption Spectroscopy (UTAS) ...................................... 43

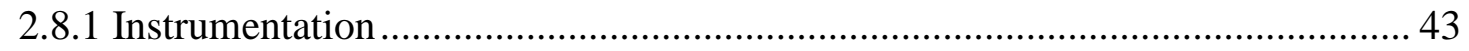

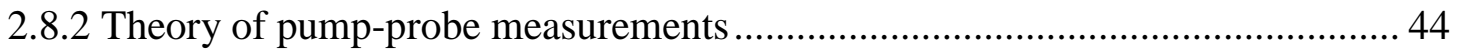

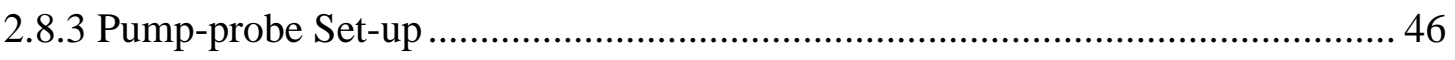

2.8.4 $860 \mathrm{~nm}$ Probe for Decay Measurements.......................................................... 48

\section{CHAPTER 3}

COVALENT MODIFICATION OF PHOTOANODES FOR STABLE

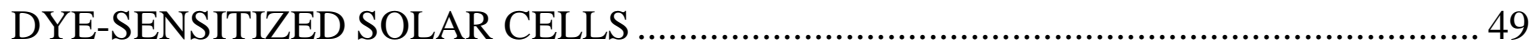

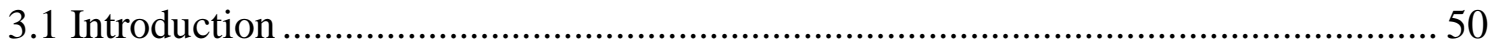

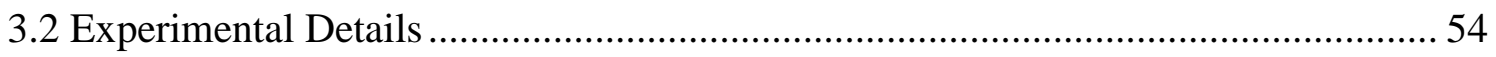

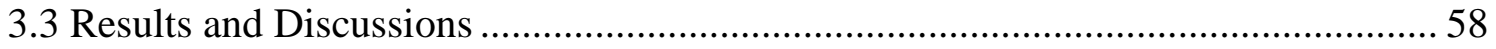

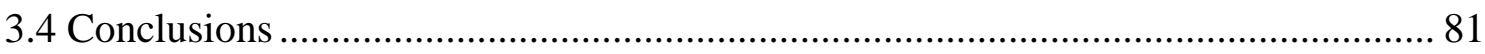

\section{CHAPTER 4}

SPECTROSCOPIC INVESTIGATION OF PHOTOINDUCED CHARGE-TRANSFER PROCESSES IN FTO/TiO $/$ /N719 PHOTOANODES WITH AND WITHOUT COVALENT ATTACHMENT THROUGH SILANE-BASED LINKERS ..................... 85

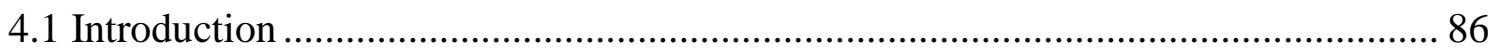




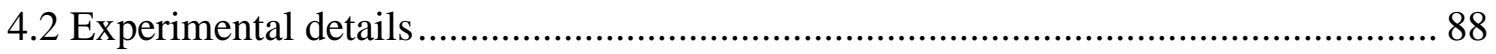

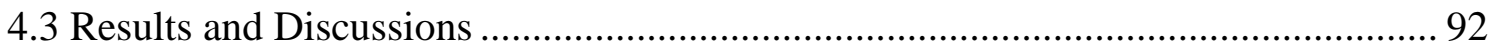

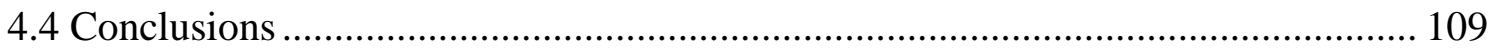

\section{CHAPTER 5}

INCREASED EFFICIENCY OF DYE-SENSITIZED SOLAR CELLS USING A RARE EARTH OXIDE/TITANIA COMPOSITE ACCEPTOR …………………………........ 112

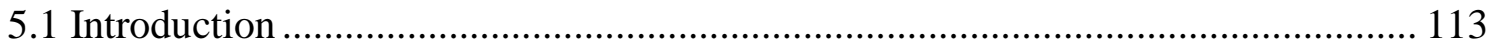

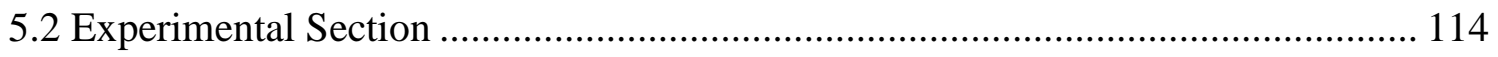

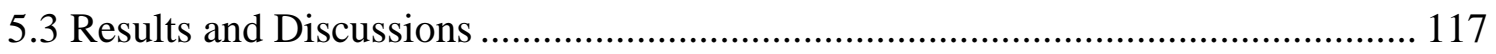

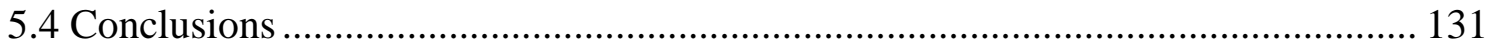

CHAPTER 6

IMPROVED PHOTOVOLTAIC PERFORMANCE OF DYE-SENSITIZED SOLAR CELLS BY USING CHEMICALLY-LINKED GOLD NANOPARTICLES ................. 132

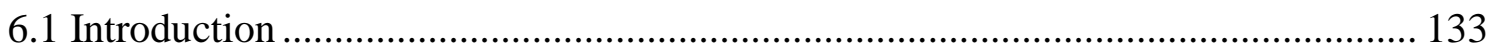

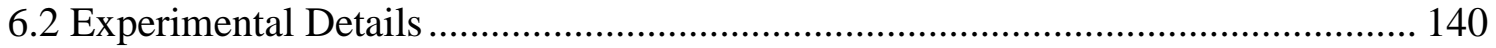

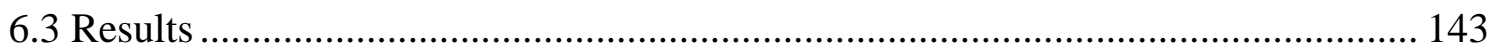

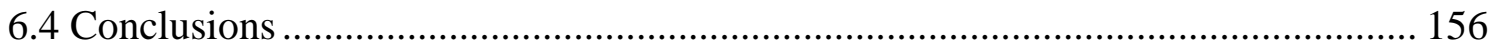

CHAPTER 7

GLOBAL CONCLUSIONS AND FUTURE DIRECTIONS ….................................... 158

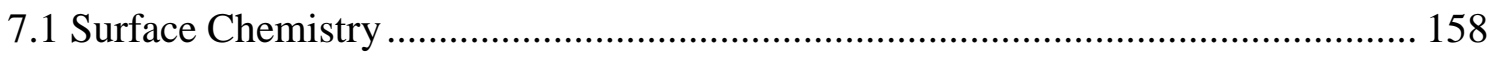

7.2 Combination of Bulk and Nanoparticles .............................................................. 159

7.3 Nanostructured Materials ................................................................................. 160

7.4 Plasmon Enhanced Charge Transfer Dynamics at Nanoscale ................................ 160

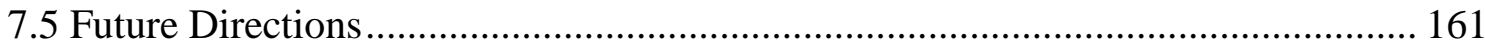

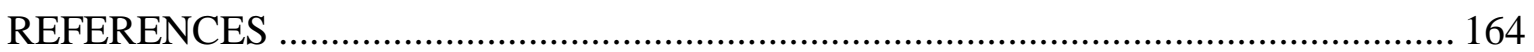

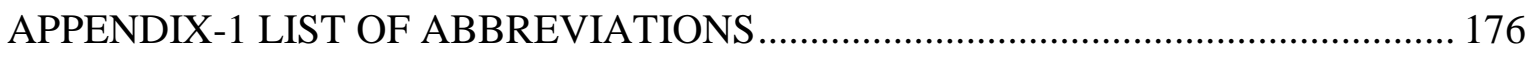

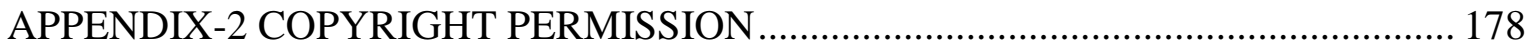

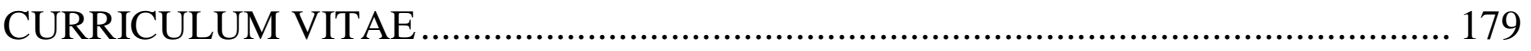




\section{LIST OF TABLES}

Table 1.1. Renewable energy sources and their availability.

Table 3.1. ATR-FTIR and UV-vis absorbance values of the various photoanodes prepared

in this study. The absorbance values are related to the dye coverage............................. 66

Table 4.1. Fit parameters for TAAPS kinetics............................................................ 96

Table 5.1. Summary of total number of photoanodes fabricated in the laboratory. ........ 121

Table 6.1. General survey of literature for the study of PEC parameters of a DSSC..... 138

Table 6.2. General survey of literature for the study of PEC parameters of a DSSC continue from table one. These are reported for low efficiency................................. 139

Table 6.3. PEC parameters for screen printed PAs................................................. 148

Table 6.4. PEC parameters for all AuNP-modified and conventional PAs.................... 150 


\section{LIST OF FIGURES}

Figure 1.1. A typical dye-sensitized solar cell (A) and path of electrons at the molecular level (B). Two electrons are involved in the $\mathrm{I}^{-} / \mathrm{I}_{3}{ }^{-}$electrolyte system............................. 4

Figure 1.2. Basic operation of a DSSC with energy level diagram and temporal

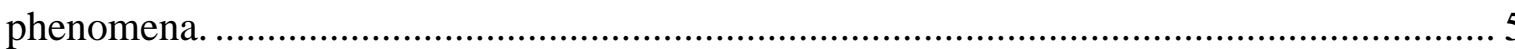

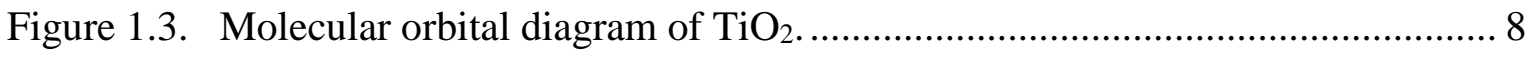

Figure 1.4. Some common dyes used in DSSCSs. A detail list can be found in

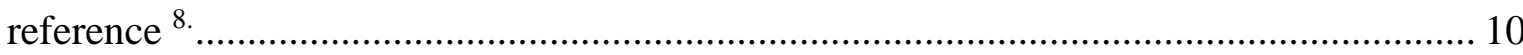

Figure 1.5. Molecular sketch of N719 and metal to ligand charge transfer (MLCT) mechanism. Real molecular sketch is on the left...................................................... 11

Figure 1.6 A solar cell battery (A), J-V curve produced by a device at operating condition (B). 15

Figure 2.1. Deposition of a $\mathrm{TiO}_{2}$ blocking layer on FTO slides starting from the very beginning stage of preparation, including dicing, cleaning, and $\mathrm{TiCl}_{4}$ treatment.............. 23

Figure 2.2. Preparation of the $\mathrm{TiO}_{2}$ active layer on FTO by the doctor blade method..... 24

Figure 2.3. Single layer deposition of the scattering layer on top of the active layer....... 25

Figure 2.4. Sintering of $\mathrm{TiO}_{2}$ on $\mathrm{FTO}$ at $500^{\circ} \mathrm{C}$. The top figure depicts the inside of the oven containing $\mathrm{FTO} / \mathrm{TiO}_{2}$ electrodes on a crystalizing dish. 26 
Figure 2.5. Post treatment of $\mathrm{FTO} / \mathrm{TiO}_{2}$ samples Figure (A) and sintering at $500{ }^{\circ} \mathrm{C}$ Figure (B). The top figure on Figure (A) is crystallizing dish with samples dipped into $40 \mathrm{mM}$ $\mathrm{TiCl}_{4}$ aqueous solution. This is actually inside the water bath. The Figure (B) shows the samples sintered at $500{ }^{\circ} \mathrm{C}$ inside the oven. The top part of the figure shows inside of the oven where samples were sintered.

Figure 2.6. Sensitization of $\mathrm{FTO} / \mathrm{TiO}_{2}$ electrodes with N719. Red picture is from nonconductive part. The scattering layer does not absorb dye. For simplicity dye solution in a vial is shown here

Figure 2.7. Design of the device with a hole on the CE (A), and photograph of the hole and sealing performed on the glass slides (B). 28

Figure 2.8. Sealing by thermal press (A), electrolyte filling by vacuum (B), and real final device $(\mathrm{C})$. 30

Figure 2.9. Sandwich cell (A) and Beaker cell (B). 31

Figure 2.10. Equivalent circuit for EIS used in dye-sensitized solar cells. Real device (A), equivalent circuit (B), and Nyquist diagram for the device (C). 38

Figure 2.11. General sketch of a surface profilometer. 39

Figure 2.12. Calculation of PEC parameters. Device connected to a potentiostat (A) and I$\mathrm{V}$ curve of the device after illumination with light (B). 41

Figure 2.13. Homemade arrangement of sample and silicon reference for I-V measurements

Figure 2.14. Measurement of I-V curves using solar simulated light 43

Figure 2.15. Pump-probe experimental procedure for the measurement of charge injection dynamics. 
Figure 3.1 ATR-FTIR spectra for $\mathrm{FTO} / \mathrm{TiO}_{2}$ (red) and $\mathrm{FTO} / \mathrm{TiO}_{2} / \mathrm{APTES}$ (green)

Figure 3.2. XPS spectra of $\mathrm{FTO} / \mathrm{TiO}_{2}$ (red) and $\mathrm{FTO} / \mathrm{TiO}_{2} / \mathrm{APTES}$ (green). Other colors are the fitted curves. (A) General survey, (B) carbon peak (C1S, $284.1 \mathrm{eV}),(\mathrm{C})$ oxygen (O1S, 529-531 eV), (D) nitrogen (N1S, free $401 \mathrm{eV}, \mathrm{H}$-bonded $398 \mathrm{eV})$, (E) silicon (2P, $110 \mathrm{eV})$, and titanium $\left(\mathrm{TiP}_{1 / 2}, \mathrm{TiP}_{3 / 2} 467\right.$ and $462 \mathrm{eV}$, respectively). .............................. 56

Figure 3.3 Comparison of I-V curves for all six types of photoanodes in (A) sandwich cells and (B) glass cells using $\mathrm{P} 25$ as the $\mathrm{TiO}_{2}$ paste for both types of cells. The average efficiency values and standard deviations are from three samples for each types of photoanodes.

Figure 3.4. The efficiency of DSSCs prepared with the indicated photoanodes using the glass cell set-up as a function of photoanode exposure time in air. The $\mathrm{TiO}_{2}$ paste was $\mathrm{P} 25$.

Figure 3.5. ATR-FTIR spectroscopy of the six different photoanodes prepared in this study using Dyesol $\mathrm{TiO}_{2}$ paste.

Figure 3.6. ATR-FTIR spectrum of benzoic acid (BA) attached to FTO/TiO $2 / \mathrm{APTES}$ from a dichloromethane solution containing DCC and DMAP. The peak at $1646 \mathrm{~cm}^{-1}$ is consistent with an amide I stretch, confirming covalent amide bond attachment. This peak is similar to the covalent amide bond stretch observed for N719 dye attached to $\mathrm{FTO} / \mathrm{TiO}_{2} / \mathrm{APTES}$ in a similar manner.

Figure 3.7. ATR-FTIR spectrum of N719 dye attached to $\mathrm{TiO}_{2}$ from a dichloromethane solution containing DMAP only (no DCC). The peak at $1648 \mathrm{~cm}^{-1}$ is still present, showing that DMAP alone can lead to the $\mathrm{TiO}_{2} /$ Dye interaction responsible for this peak.

Figure 3.8. UV-vis spectroscopy of $\mathrm{N} 719$ dye adsorbed on Dyesol $\mathrm{TiO}_{2}$ paste for the six photoanodes.

Figure 3.9. Air stability test. (A) Top: ATR-FTIR spectra of conventional FTO/TiO $2 /$ Dye $^{2}$ (NC-1), red 1st day and green after 20 days. Below: ATR-FTIR of covalent 
$\mathrm{FTO} / \mathrm{TiO}_{2} / \mathrm{APTES} / \mathrm{Dye}(\mathrm{C}-3)$, blue $1^{\text {st }}$ day and cyan after 20 days. (B) UV-vis spectra of conventional $\mathrm{FTO} / \mathrm{TiO}_{2} / \mathrm{Dye}$ (NC-1), red 1st day and green after 20 days. (C) UV-vis of covalent FTO/TiO $2 /$ APTES/Dye (C-3), blue $1^{\text {st }}$ day and cyan after 20 days. Photoanodes were stored in a closed box filled with air.

Figure 3.10. Photograph (A) FTO/TiO $/$ Dye(NC-1), (B) FTO/TiO $/$ /Dye (NC-2), (C) $\mathrm{FTO} / \mathrm{TiO}_{2} /$ Dye (NC-3), and (D) FTO/TiO $2 / \mathrm{APTES}_{\text {Dye }}$ (C-3) before (0 days) and after 20 days of storage in open air. The plot below shows a graph of the blue shift of the MLCT band around $525-545 \mathrm{~nm}$ for $\mathrm{N} 719$ dye adsorbed on $\mathrm{TiO}_{2}$ as a function of storage time in air for the $\mathrm{FTO} / \mathrm{TiO}_{2} / \mathrm{Dye}(\mathrm{NC}-1)$ "red" and $\mathrm{FTO} / \mathrm{TiO}_{2} / \mathrm{APTES} /$ Dye (C-3) "blue" photoanodes. 73

Figure 3.11. (A) ATR-FTIR spectra of conventional $\mathrm{FTO} / \mathrm{TiO}_{2} / \mathrm{Dye} /(\mathrm{NC}-1)$ and covalent $\mathrm{FTO} / \mathrm{TiO}_{2} / \mathrm{APTES} / \mathrm{Dye}(\mathrm{C}-3)$ photoanodes before and after heating in open air at $140{ }^{\circ} \mathrm{C}$ for $3 \mathrm{~h}$ and simultaneously illuminating with UV light. (B) and (C) are UV-vis spectra of conventional (FTO/TiO $/$ /Dye/(NC-1)) and covalent (FTO/TiO $/ 2$ APTES/Dye(C-3)) photoanodes before and after heating in open air at $140{ }^{\circ} \mathrm{C}$ for $3 \mathrm{~h}$ and simulataneouly exposing to UV light. The samples were exposed to both $254 \mathrm{~nm}$ and $364 \mathrm{~nm}$ UV-light.

Figure 3.12. ATR-FTIR spectra (above) of non-covalent $\mathrm{FTO} / \mathrm{TiO}_{2} / \mathrm{Dye}^{\mathrm{NC}-3}$ ) and covalent $\mathrm{FTO} / \mathrm{TiO}_{2} / \mathrm{APTES} / \mathrm{Dye}$ (C-3) photoanodes before and after soaking in $28 \mathrm{mM}$ solution of acetic acid in acetonitrile for $12 \mathrm{~h}$. The photographs show a non-covalent photoanode $\mathrm{FTO} / \mathrm{TiO}_{2} / \mathrm{Dye}$ (NC-3) (upper) and a covalent photoanode FTO/TiO $/$ APTES/Dye (C-3) (lower) before and after same acetic acid treatment. While the ATR-FTIR spectra are initially very similar, the chemistry of these two photoanodes is

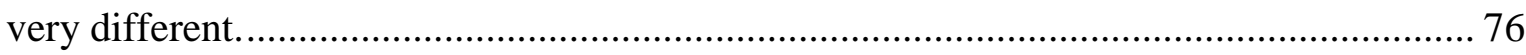

Figure 3.13. (A) Photocurrent increased in covalent PAs after treating with acetic acid. (B)Both types of PAs, covalent and non-covalent did not show stability towards base. (C) Sketch of monolayer and multilayer on $\mathrm{TiO}_{2}$ after sensitization with $\mathrm{N} 719$ dye. In ACN:tbutanol solution, N719 is supposed to make a monolayer. (D) After multilayer was removed, covalent PAs showed an increase in efficiency from 3\% to $4 \%$, a $33 \%$ increase. 77

Figure 3.14. (A) ATR-FTIR spectra of conventional FTO/TiO $2 /$ Dye (NC-1) and covalent FTO/TiO $/$ /APTES/Dye (C-3) photoanodes before and after soaking in water for $1 \mathrm{~h}$. UV- 
vis spectra of (B) conventional $\mathrm{FTO} / \mathrm{TiO}_{2} /$ Dye (NC-3) photoanodes before and after soaking in water for $1 \mathrm{~h}$.

Figure 3.15. I-V curves and dark currents for covalent photoanodes (blue), non-covalent photoanodes (red) (A), UV-vis of the same photoanodes (B) and ATR-FTIR spectroscopy of the same photoanodes (C). 80

Figure 3.16. (A) ATR-FTIR data of $\mathrm{FTO} / \mathrm{TiO}_{2} /$ Dye functionalized with octylamine covalently through an amide bond on the first day of preparation "Day 1" and the third day of exposure to air "Day 3". The dye is not stable based on the many changes in the IR

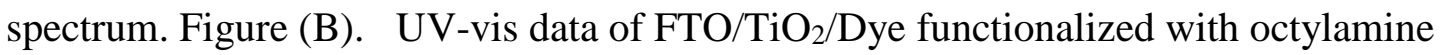
covalently through an amide bond on the first day of preparation "Day 1" and the third day of exposure to air "Day 3". Figure (C). I-V curves of FTO/TiO $2 /$ Dye functionalized with octylamine covalently through an amide bond on the first day of preparation "Day 1" and the third day of exposure to air "Day 3".

Figure 3.17. ATR-FTIR spectra of (A) $\mathrm{FTO} / \mathrm{TiO}_{2} / \mathrm{Dye}(\mathrm{NC}-1)$ and (B)

FTO/TiO 2 /APTES/Dye (C-3) on the initial day of preparation and after 4 months and 6 months of storage in air.

Figure 4.1. Schemes of the $\mathrm{N} 719$ dye attached to $\mathrm{TiO}_{2}$ films by (a) the conventional direct method, (b) a covalent APTES linker, and (c) a covalent aromatic APhS linker, and the corresponding ATR-FTIR spectra of the photoanodes measured before (red lines) and after (blue lines) exposure to air for 20 days (d-f). In (a)-(c), only the single attachment situation is shown (see text for more details). Spectra are offset for clarification. Note the loss in the $\mathrm{CN}$ stretch peak at $2103 \mathrm{~cm}^{-1}$ in the conventional photoanode spectra after air exposure. ${ }^{110}$

Figure 4.2. Steady-state UV/visible absorption spectra of (a) N719 dye in ACN:t-butanol (1:1) solution and (b) FTO/TiO $/ \mathrm{N}_{2} 79$, (c) FTO/TiO $/ \mathrm{APTES}_{2}$ N719, and (d) $\mathrm{FTO} / \mathrm{TiO}_{2} / \mathrm{APhS} / \mathrm{N} 719$ photoanodes. 94

Figure 4.3. (a) Normalized transient absorption kinetics observed with the $530 \mathrm{~nm}$ pump wavelength and $860 \mathrm{~nm}$ probe wavelength of $\mathrm{FTO} / \mathrm{TiO}_{2} / \mathrm{N} 719, \mathrm{FTO} / \mathrm{TiO}_{2} / \mathrm{APTES} / \mathrm{N} 719$, and $\mathrm{FTO} / \mathrm{TiO}_{2} / \mathrm{APhS} / \mathrm{N} 719$ photoanodes. (b)-(d) illustrate the kinetics up to $100 \mathrm{ps}$ and the biexponential fits. 
Figure 4.4. Transient absorption 2D time-wavelength contour plots of (a) N719 dye in $\mathrm{ACN}$ and tert-butanol (1:1) solution and (b) $\mathrm{FTO} / \mathrm{TiO}_{2} / \mathrm{N} 719$, (c) $\mathrm{FTO} / \mathrm{TiO}_{2} / \mathrm{APTES}_{\mathrm{N}} 719$, and (d) $\mathrm{FTO} / \mathrm{TiO}_{2} / \mathrm{APhS} / \mathrm{N} 719$ photoanodes.

Figure 4.5. Transient absorption spectra at $\Delta \mathrm{t}=0.5 \mathrm{ps}, 10 \mathrm{ps}, 100 \mathrm{ps}, 500 \mathrm{ps}$, and $1 \mathrm{~ns}$ of (a) N719 dye in ACN and tert-butanol (1:1) solution and (b) FTO/TiO $/ 2719$, (c) $\mathrm{FTO} / \mathrm{TiO}_{2} / \mathrm{APTES}_{\mathrm{N} 719}$, and (d) $\mathrm{FTO} / \mathrm{TiO}_{2} / \mathrm{APhS} / \mathrm{N} 719$ photoanodes. Two broad spectral regions, ground-state bleach (GSB) and excited-state absorption (ESA), are indicated. . 100

Figure 4.6. Normalized transient kinetics observed with the $530 \mathrm{~nm}$ pump wavelength and (a) $470 \mathrm{~nm}$, (b) $650 \mathrm{~nm}$, and (c) $690 \mathrm{~nm}$ probe wavelengths of N719 dye in ACN and

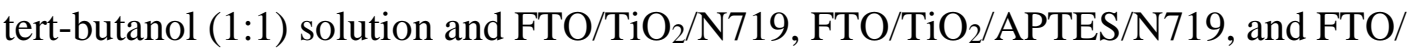
$\mathrm{TiO}_{2} / \mathrm{APhS} / \mathrm{N} 719$ photoanodes. The delay time step size is $3 \mathrm{ps}$.

Figure 4.7. Nrobe wavelength of (a) $\mathrm{FTO} / \mathrm{TiO}_{2} / \mathrm{N} 719$, (b) $\mathrm{FTO} / \mathrm{TiO}_{2} / \mathrm{APTES} / \mathrm{N} 719$, and (c) $\mathrm{FTO} / \mathrm{TiO}_{2} / \mathrm{APhS} / \mathrm{N} 719$ photoanodes. The delay time step size is $20 \mathrm{fs}$. 104

Figure 5.1. Images of the surface of $\mathrm{TiO}_{2}(\mathrm{~A}, \mathrm{C}$, and $\mathrm{E})$ and $5 \% \mathrm{Nd}_{2} \mathrm{O}_{3} / \mathrm{TiO}_{2}(\mathrm{~B}, \mathrm{D}$ and $\mathrm{F})$ photoanodes. A-B show optical microscope images while C-F show scanning electron microscope images

Figure 5.2. Optical reflectance measurements of a $\mathrm{TiO}_{2}$ film (red) and a $\mathrm{TiO}_{2}$ film doped with $5 \% \mathrm{Nd}_{2} \mathrm{O}_{3}$ microparticles (blue). The band edge is similar for both films and the transitions for the $\mathrm{Nd}_{2} \mathrm{O}_{3}$ are clearly observed in the visible range for the hybrid film as noted by the arrows.

Figure 5.3. Current-density versus bias for (A) $\mathrm{Nd}_{2} \mathrm{O}_{3}$ doped and (B) undoped photoanodes measured under 1.5 AM illumination. Different batches are shown as different colored lines: Batch 1(yellow), 2 (not shown), 3 (black), 4 (blue), 5 (green) and 6 (red). (C-F) Box plots show (C) short circuit current, (D) efficiency, (E) fill factor, and (F) open circuit voltage for each of the six batches. Solid blocks show $\mathrm{Nd}_{2} \mathrm{O}_{3}$ doped samples, and hashed blocks show undoped samples. 120

Figure 5.4. Cyclic voltammetry of (A) $\mathrm{FTO} / \mathrm{TiO}_{2}-5 \% \mathrm{Nd}_{2} \mathrm{O}_{3} / \mathrm{Dye}$, and (B) $\mathrm{FTO} / \mathrm{TiO}_{2} /$ Dye. The devices were illuminated by $35 \mathrm{~mW} / \mathrm{cm} 2$ optical fiber light. The 50 scans at $20 \mathrm{mV} / \mathrm{s}$ 
were performed by using two terminal contacts. The scan window of the voltage was from 0 to $-1 \mathrm{~V}$. The sign of the photocurrent and photovoltage are reversed in the plot. 122

Figure 5.5. Photoelectrochemical parameters measured over a time period of 40 days of a well-sealed dye-sensitized solar cell device with a $\mathrm{FTO} / \mathrm{TiO}_{2}-5 \% \mathrm{Nd}_{2} \mathrm{O}_{3} /$ Dye photoanode.

Figure 5.6. Current voltage characteristics for DSSCs made with anodes containing varying percent weight concentrations of $\mathrm{Nd}_{2} \mathrm{O}_{3}$. Inset: Efficiency as a function of $\mathrm{Nd}_{2} \mathrm{O}_{3}$ percent weight concentration.

Figure 5.7. A plot of dye absorbance as a function of $\mathrm{Nd}_{2} \mathrm{O}_{3} \%$ concentration for $\mathrm{TiO}_{2}$ films doped with $\mathrm{Nd}_{2} \mathrm{O}_{3}$ microparticles. The dye absorbance, or coverage, reaches a maximum at $5 \%$ doping by weight (before calcination). The efficiency of the photoanode is largest at the same percentage.

Figure 5.8. Optical images of (A) nanoscale $\mathrm{TiO}_{2}$ only and (B) nanoscale $\mathrm{TiO}_{2}$ containing $5 \%$ microscale $\mathrm{TiO}_{2}$ particles. (C) Dye absorbance on nanoscale $\mathrm{TiO}_{2}$ only (red) and nano $\mathrm{TiO}_{2}+5 \%$ micro $\mathrm{TiO}_{2}$ (blue) films. (D) J-V curves under $1.5 \mathrm{AM}$ solar light showing the efficiency of the dye-coated nano $\mathrm{TiO}_{2}$ films (solid red) and nano $\mathrm{TiO}_{2}+5 \%$ micro $\mathrm{TiO}_{2}$ (solid blue) films with the dark $\mathrm{J}-\mathrm{V}$ curves shown as the corresponding dashed lines. ... 125

Figure 5.9. (A) UV-vis absorbance spectra for anodes made with $5 \% \mathrm{Nd}_{2} \mathrm{O}_{3} / \mathrm{TiO}_{2}$ composite anodes (green solid line) and undoped $\mathrm{TiO}_{2}$ anodes (red dashed line). (B) Absorbance as a function of dye soaking time. (C) Absorbance for reduced dye soaking time. (D) Light current voltage characteristics for the dye coverage corresponding to $(\mathrm{C})$.

Figure 5.10. Electron impedance spectra for photoanodes containing different concentrations of $\mathrm{Nd}_{2} \mathrm{O}_{3}$ in the photoanodes.

Figure 5.11. ATR-FTIR spectra of $\mathrm{FTO} / \mathrm{TiO}_{2}-5 \% \mathrm{Nd}_{2} \mathrm{O}_{3}$ (dark cyan) and $\mathrm{FTO} / \mathrm{TiO}_{2}$ (red) photoanodes. $\mathrm{OH}$ site peak at $3695 \mathrm{~cm}^{-1}$ disappeared and $\mathrm{H}_{2} \mathrm{O}$ adsorbed mode is diminished in $\mathrm{FTO} / \mathrm{TiO}_{2}-5 \% \mathrm{Nd}_{2} \mathrm{O}_{3}$ photoanodes. 129 
Figure 5.12. (A) Cyclic Voltammograms (CV) of ITO, ITO/TiO 2 , and $\mathrm{ITO} / \mathrm{TiO}_{2}$ $5 \% \mathrm{Nd}_{2} \mathrm{O}_{3}$ in $0.1 \mathrm{M} \mathrm{KCl}$ solution at $\mathrm{pH}$. (B) Normalized photovoltage $\left(\mathrm{V}_{\mathrm{ph}}\right)$ decay curve of $\mathrm{FTO} / \mathrm{TiO}_{2}$ (red), and $\mathrm{FTO} / \mathrm{TiO}_{2}-5 \% \mathrm{Nd}_{2} \mathrm{O}_{3}$ (dark cyan) anodes in a beaker cell with three terminals in $0.5 \mathrm{M} \mathrm{KSCN}$ aqueous solution.

Figure 5.13. UV-vis absorption of $\mathrm{FTO} / \mathrm{TiO}_{2} / \mathrm{Dye}$ and $\mathrm{TiO}_{2}-\mathrm{Nd}_{2} \mathrm{O}_{3}(20: 1)$ nano-mesh composite (A). Incident photon-to-current conversion efficiency (B). I-V curve for the same photoanodes used to measure UV-vis and IPCE (C). Dye-soaking time period was 24 h. $\mathrm{TiO}_{2}$ and $\mathrm{Nd}_{2} \mathrm{O}_{3}$ modified paste had double layers made by the doctor blade method. The thickness was $11 \mu \mathrm{m}$.

Figure 6.1. SEM images of (A) $\mathrm{TiO}_{2}$ and (B) $\mathrm{TiO}_{2} / \mathrm{APTES} / \mathrm{AuNPs}$ .145

Figure 6.2. AFM images of (A) $\mathrm{TiO}_{2}$ and (B) $\mathrm{TiO}_{2} / \mathrm{APTES} / \mathrm{AuNPs}$ 145

Figure 6.3. ATR-FTIR spectroscopy of $\mathrm{FTO} / \mathrm{TiO}_{2}$ (red), $\mathrm{FTO} / \mathrm{TiO}_{2} / \mathrm{APTES}$ (green), and FTO/TiO 2 /APTES/AuNPs (blue). 146

Figure 6.4. ATR-FTIR spectroscopy of conventional FTO/TiO $/$ Dye (red) FTO/TiO $/$ APTES /Dye (green) and FTO/TiO $2 /$ APTES/AuNPs/Dye (blue)

Figure 6.5. UV-vis spectra (A) and $\mathrm{I}-\mathrm{V}$ curves (B) of $\mathrm{FTO} / \mathrm{TiO}_{2} / \mathrm{Dye}$ (red) and FTO/TiO $2 /$ APTES/AuNPs/Dye (blue) photoanodes. The $\mathrm{TiO}_{2}$ paste was made from DSL $18 \mathrm{NR}-\mathrm{T}$ by screen printing, the area was $0.25 \mathrm{~cm}^{2}$, and there was no scattering layer to allow UV-vis analysis. The efficiency of the DSSC modified with Au NPs showed a 35\% increase in efficiency by increasing both photocurrent and photovoltage.

Figure 6.6. (A) Photocurrent-photovoltage plots by batch number for AuNP-modified photoanodes and (B) $\mathrm{TiO}_{2}$ photoanodes. Each batch contains two or three devices and the plots contain average values of I-V curve from each batch, Blue, green, red, pink, and cyan colors denoted batch numbers $1,2,3,4$, and 5 , respectively. 150

Figure 6.7. Bar plot of current density (A), photovoltage (B), efficiency in percentage (C), and fill factor (Figure D) of linker assisted AuNP-modified photoanodes (blue) and $\mathrm{TiO}_{2}$ only photoanodes (red). Increase in efficiency of the devices is not only due to the 
enhancement of current but also due to the increase of photovoltage. A few devices have lower current densities, but, the other parameters $\left(\mathrm{V}_{\mathrm{oc}}, \eta\right.$, and $\left.\mathrm{FF}\right)$ remained the same... 151

Figure 6.8. Figure (A) UV-vis spectra of $\mathrm{FTO} / \mathrm{TiO}_{2} / \mathrm{Dye}$ (red), $\mathrm{FTO} / \mathrm{TiO}_{2} / \mathrm{APTES} / \mathrm{Dye}$ (green), and $\mathrm{FTO} / \mathrm{TiO}_{2} / \mathrm{APTES} / \mathrm{AuNP}$ /Dye (blue) PAs, (B) IPCE of the corresponding photoanodes, and (C) I-V curves of the same devices. Dye-loading was the same for all PAs, and the area of all PAs was $0.30 \mathrm{~cm}^{2}$. The dash lines are dark I-V curves.

Figure 6.9. (A) UV-vis of aliquots of $\mathrm{N} 719$ dye desorbed from $\mathrm{FTO} / \mathrm{TiO}_{2} / \mathrm{Dye}$ (red), $\mathrm{FTO} / \mathrm{TiO}_{2} \mathrm{APTES} / \mathrm{Dye}$ (green), and $\mathrm{FTO} / \mathrm{TiO}_{2} / \mathrm{APTES} / \mathrm{Au} / \mathrm{Dye}$ (blue). (B) I-V curves $\mathrm{FTO} / \mathrm{TiO}_{2} /$ Dye (red), FTO/TiO ${ }_{2} \mathrm{APTES} /$ Dye (green), and FTO/TiO $2 / \mathrm{APTES} / \mathrm{Au} / \mathrm{Dye}$ (blue). The I-V curves were measured from beaker cells.

Figure 6.10. EIS plot for FTO/TiO 2 /Dye (red solid square), FTO/TiO $2 /$ APTES /Dye (green solid circle, and FTO/TiO $/$ APTES/AuNPs/Dye (blue solid sphere) photoanodes having similar dye coverage.

Figure 6.11. Comparisons of injection time scales for $\mathrm{FTO} / \mathrm{TiO}_{2} /$ Dye (red, traditional), $\mathrm{FTO} / \mathrm{TiO}_{2} / \mathrm{APTES} / \mathrm{Dye}$ (green), and $\mathrm{FTO} / \mathrm{TiO}_{2} / \mathrm{APTES} / \mathrm{AuNPs} / \mathrm{Dye}$ (blue). T denotes the ultrafast time scale for excited electrons to go from LUMO to conduction band of $\mathrm{TiO}_{2}$. The ultrafast injection dynamics are the fastest in Au NPs modified photoanodes. 155 


\section{LIST OF SCHEMES}

Scheme 2.1. Preparation of photoanodes for dye-sensitized solar cells.

Scheme 2.2. Fabrication of dye-sensitized solar cells. 22

Scheme 3.1. Different possible interactions between $\mathrm{TiO}_{2}$ and $\mathrm{N} 719$ dye molecule........ 52

Scheme 3.2. Preparation of different types of photoanodes and their nomenclature. ....... 59

Scheme 3.3. Possible non-covalent interactions between dye and FTO/TiO $2 / A P T E S . . . .60$

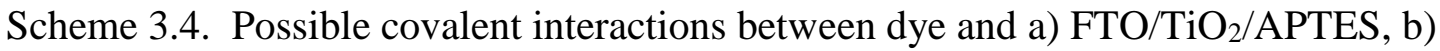
$\mathrm{FTO} / \mathrm{TiO}_{2}$ when dye molecules are attached from a solution containing DCC and DMAP. 60

Scheme 5.1. Preparation of $\mathrm{Nd}_{2} \mathrm{O}_{3}$ doped photoanodes. 115

Scheme 6.1. Illustration of potential dye location on $\mathrm{FTO} / \mathrm{TiO}_{2}, \mathrm{FTO} / \mathrm{TiO}_{2} / \mathrm{APTES}$, and $\mathrm{FTO} / \mathrm{TiO}_{2} / \mathrm{APTES} / \mathrm{Au} \mathrm{NPs}$ 144

Scheme 6.2. Illustration of potential interactions of dye in $\mathrm{FTO} / \mathrm{TiO}_{2}, \mathrm{FTO} / \mathrm{TiO}_{2} / \mathrm{APTES}$, and $\mathrm{FTO} / \mathrm{TiO}_{2} / \mathrm{APTES} / \mathrm{Au} \mathrm{NPs}$. 


\section{CHAPTER 1}

\section{INTRODUCTION}

\subsection{Global Energy Demand Scenario}

The present global population has reached over 7.2 billion and the total population on earth will exceed 9.6 billion by $2050 .{ }^{1}$ The total energy consumption by human society was 13 TW in 2005. The annual growth of energy consumption by human society is currently $1.5 \%$. Considering the present status of population growth and energy consumption, the global demand is expected to double ( 30 TW) by 2050 and quadruple ( 56 TW) by the end of the century. ${ }^{2}$ Economically strong countries like China, India, and Brazil are producing more energy-hungry populations than other countries, and they have already started making additional power plants every week to satisfy the energy needed for such huge populations in the future. ${ }^{3}$

\subsection{Available Energy Resources}

There are two types of energy resources available for humans to use: non-renewable and renewable. Almost $93 \%$ of the total energy demand, which has exponentially increased after the industrial revolution, comes from non-renewable energy resources such as fossil fuels (oil), natural gas, coal, and nuclear energy. Only a tiny fraction (7\%) of total energy 
demand is supplied by renewable energy sources such as solar, wind, bio-fuels, hydropower, geothermal, and ocean energy. ${ }^{2,4}$ The current estimation of global reserves for fossil fuels, natural gas, and coal suggest that fossil fuel has the potential to provide continuous energy for up to 54 years, natural gas up to 63 years, and coal up to 107 years. ${ }^{5,6}$ There is still room for continuing production of liquid fuels like fossil fuel from coal even after the complete consumption of fossil fuels. Nevertheless, carbon-free energy resources are crucial for sustainable development of the global society without degradation of the environment.

Table 1.1 shows the various forms of renewable energy and their availability. ${ }^{2}$

The sun is delivering $1.2 \times 10^{5} \mathrm{TW}$ of solar energy annually to the earth. Due to the physical structure of the earth and the availability of sun light during the day, only $0.5 \%(\sim 600 \mathrm{TW})$ can be extracted practically for electrical energy conversion. Even $10 \%$ of this $600 \mathrm{TW}$,

Table 1.1. Renewable energy sources and their availability.

\begin{tabular}{|c|c|c|c|}
\hline & $\begin{array}{c}\text { Current } \\
\text { Use (TW) }\end{array}$ & $\begin{array}{c}\text { Current } \\
\text { Use (\%) }\end{array}$ & $\begin{array}{c}\text { Theoretical } \\
\text { Potential (TW) }\end{array}$ \\
\hline Hydropower & 0.0792 & 36 & 0.50 \\
\hline Biomass energy & 0.1166 & 53 & 91.96 \\
\hline Wind energy & 0.011 & 5 & 4.00 \\
\hline Solar energy & 0.0022 & 1 & 123668.19 \\
\hline Geothermal energy & 0.011 & 5 & 12.00 \\
\hline
\end{tabular}

which is $60 \mathrm{TW}$, is clearly enough to produce the energy necessary for future generations. ${ }^{7,8}$ Currently, silicon crystalline solar cells represent $90 \%$ of the market share in the renewable energy sector. However, their production cost is still not suitable for mass production to be usable for everyone and everywhere. ${ }^{9}$ There is a clear necessity for an eco-friendly solar cell, which is cheap, easily available, and applicable anywhere to keep the world free of carbon emissions. 
The theoretical photovoltage that can be obtained from a dye-sensitized solar cell is around $1.1 \mathrm{eV}$ for the most common $\mathrm{TiO}_{2}$ system. ${ }^{10}$ With a possible maximum fill factor of 0.85 and theoretical solar spectrum usable up to $940 \mathrm{~nm}$, a dye-sensitized solar can have the capacity to produce a power conversion efficiency (PCE) above $20 \% .{ }^{11}$ In a short span of time, dye-sensitized solar cell technology has shown a sharp increase in device efficiency from 0.5 to $13.2 \%$; and based on its technology, perovskite solar cells are offering efficiency above $20 \%$, although they have the serious issues of toxicity and long-term stability. ${ }^{12}$ Even after a century of research, silicon solar cells are still struggling to produce energy at a reasonable cost. $20 \%$ efficient solar cells with a life time of 20 years, which can offer energy with a price similar to fossil fuels, can revolutionize the energy sector in filling the huge gap existing between energy demand and renewable energy production for sustainable development of human society globally. Dye-sensitized solar cells can be a potential candidate because of their capacity to produce high efficiency at low fabrication cost.

\subsection{Dye-sensitized Solar Cells}

\subsubsection{General Set-up and Electrochemistry}

A dye-sensitized solar cell (DSSC) is an electrochemical photovoltaic cell that mimics the principle of photosynthesis. Like chlorophyll in green plants and algae, the DSSC uses a molecular absorber, the dye, which may be an organic or organometallic chromophore to harvest sunlight and generate electricity. Figure 1.1 shows a typical dyesensitized solar cell which can act as a battery upon illumination. The dye (red) absorbs light, leading to a higher energy excited electron. The excited electron can be injected into the conduction band of the film of $\mathrm{TiO}_{2}$ nanoparticles (NPs). The electron diffuses through 
the $\mathrm{TiO}_{2}$ film to the transparent conductive substrate, fluorine doped tin-oxide (FTO), and is available for external work. The electron can also recombine with dye or directly reduce

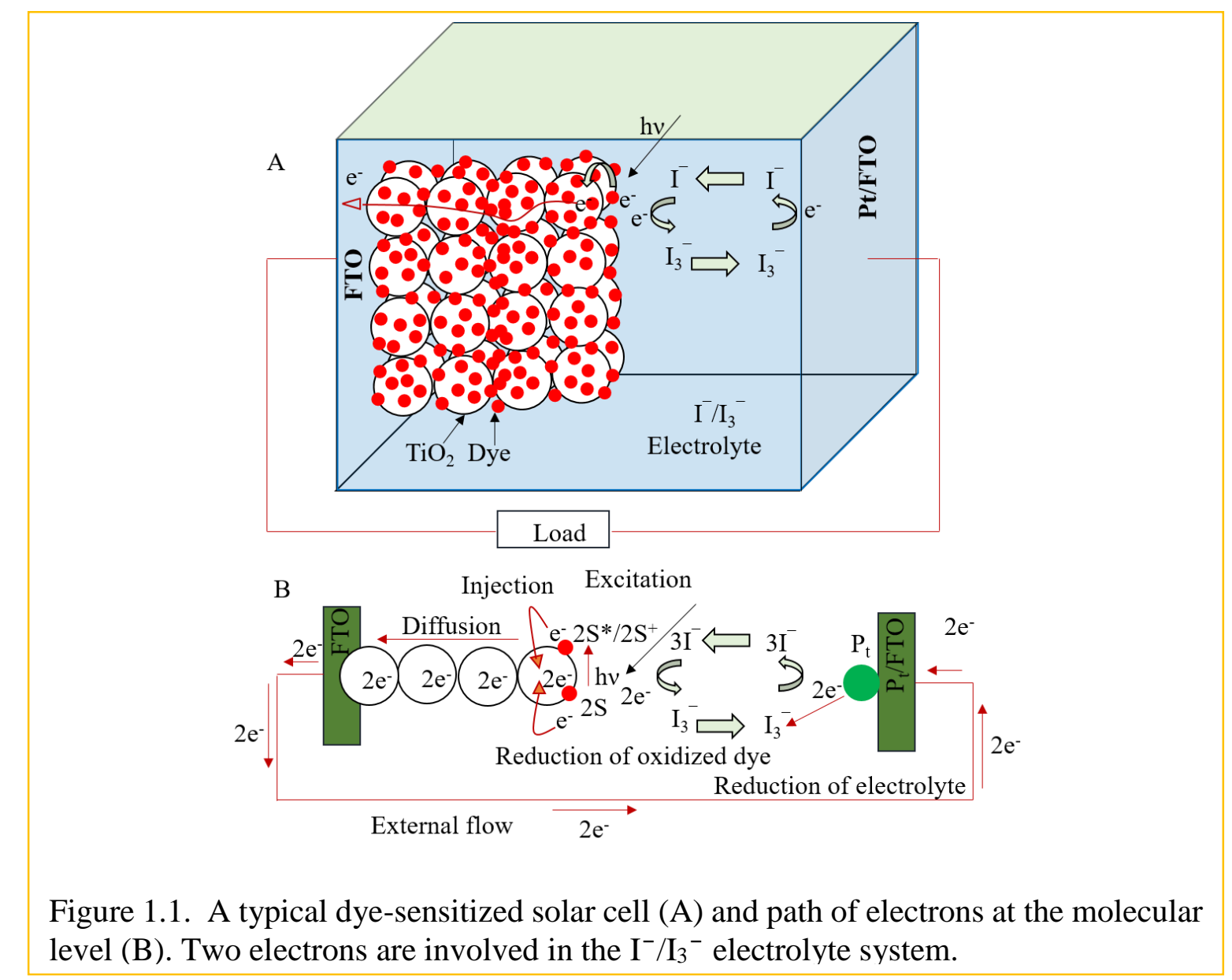

$\mathrm{I}_{3}{ }^{-}$in the electrolyte, which are both undesirable and reduce efficiency.

The detailed process is as follows: A typical DSSC consists of three components: a photoanode (PA), a redox electrolyte (usually iodide/triiodide, $\mathrm{I}^{-} / \mathrm{I}_{3}{ }^{-}$), and a counter electrode (CE, Pt coated FTO). When sunlight is absorbed by the dye, electrons are excited from the HOMO to the LUMO energy levels in the dye. Because of proximity to the conduction band (CB) of $\mathrm{TiO}_{2}$ with the LUMO of the dye, ultrafast electron transfer $(<30$ fs) occurs from the dye LUMO to the $\mathrm{TiO}_{2} \mathrm{CB}$. These injected electrons flow through the external circuit, after diffusing through mesoporous $\mathrm{TiO}_{2} \mathrm{NPs}$ and reduce $\mathrm{I}_{3}{ }^{-}$to $3 \mathrm{I}^{-}$at the 
counter electrode (CE). The reaction path is shown in Figure 1.1 and Figure 1.2. $3 \mathrm{I}^{-}$ions diffuse through the electrolyte to reach the oxidized dye molecules and reduce them back to the neutral ground state. This process repeats, and is self-sustained for the conversion of solar energy into electrical energy without any net chemical or physical changes. The maximum efficiency reported for such cells is around $11-12.3 \% .^{13,14}$

The sequence of electron-transfer events in a DSSC can be summarized as follows. ${ }^{15}$ When light strikes on the dye molecules, electronically excited $\mathrm{S} *$ dye are created, called

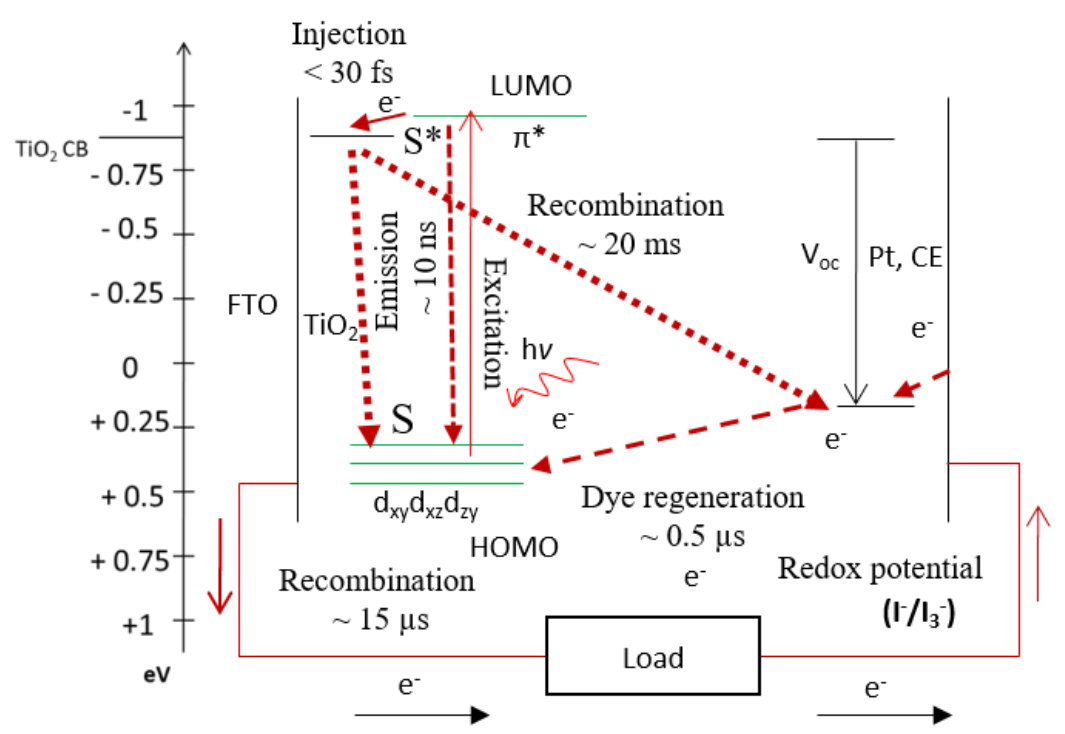

Figure 1.2. Basic operation of a DSSC with energy level diagram and temporal phenomena.

photoexcitation:

$$
2 \mathrm{~S}+\mathrm{hv} \longrightarrow 2 \mathrm{~S}^{*}
$$

(Photoexcitation).

The molecules in the excited state can decay back to the ground state by emission or undergo oxidative quenching, injecting electrons into the conduction band of $\mathrm{TiO}_{2}$. We want the latter to occur as follows.

$$
\left.2 \mathrm{~S}^{*}+\mathrm{TiO}_{2}(\mathrm{cb}) \longrightarrow 2 \mathrm{~S}^{+}+\mathrm{TiO}_{2}\left(2 \mathrm{e}^{-} \mathrm{cb}\right) \quad \text { (Photoinjection }<30 \mathrm{fs}\right) .
$$


The injected electrons travel through the mesoporous network of $\mathrm{TiO}_{2}$ particles to reach the FTO collector electrode and flow into the external circuit. The electrons reach the counterelectrode through the external circuit and reduce the triiodide $\left(\mathrm{I}_{3}^{-}\right)$in the solution.

$$
\mathrm{I}_{3}^{-}+2 \mathrm{e}^{-} \longrightarrow 3 \mathrm{I}^{-} \quad \text { (Electrochemical reduction of } \mathrm{I}_{3}^{-} \text {at } \mathrm{CE} \text { ). }
$$

The oxidized dye is then chemically reduced by $\mathrm{I}^{-}$in the electrolyte, which is known as regeneration:

$$
2 \mathrm{~S}^{+}+3 \mathrm{I}^{-} \longrightarrow 2 \mathrm{~S}+\mathrm{I}_{3}^{-} \quad \text { (Regeneration of oxidized dye, } \sim 0.5 \mu \mathrm{s} \text { ) }
$$

These processes repeat to convert sunlight into electrical energy. There are several competing undesirable pathways the electrons can take after excitation from the light.

First, the electron can simple go back to the ground state of the dye, leading the light emission as follows.

$$
\mathrm{S}^{*} \quad \longrightarrow \mathrm{S}+\mathrm{hv} \quad \text { (Emission) }
$$

There is also the possibility that after excitation and injection into $\mathrm{TiO}_{2}$, the electrons can recombine with dye or directly reduce $\mathrm{I}_{3}{ }^{-}$in the electrolyte as follows instead of going through the external circuit.

$$
\begin{array}{lll}
2 \mathrm{~S}^{+}+2 \mathrm{e}^{-}\left(\mathrm{TiO}_{2}, \mathrm{cb}\right) & 2 \mathrm{~S} \quad \text { (Recombination, } 15 \mu \mathrm{s}) \\
\mathrm{I}_{3}^{-}+2 \mathrm{e}^{-}\left(\mathrm{TiO}_{2}, \mathrm{cb}\right) & \left.\longrightarrow 3 \mathrm{I}^{-} \quad \text { (Recombination, } 20 \mathrm{~ms}\right)
\end{array}
$$

The other possible process is the transport of electrons from the back contact (FTO) to the electrolyte, which also degrades the performance of the device.

$$
\mathrm{I}_{3}^{-}+2 \mathrm{e}^{-}(\mathrm{FTO}) \longrightarrow 3 \mathrm{I}^{-} \quad(\text { Recombination })
$$

The overall effect of irradiation with sunlight is to drive the electrons through the external circuit and reduce $\mathrm{I}_{3}{ }^{-}$at the $\mathrm{CE}$ thus converting sunlight into electricity without any net 
chemical and physical changes. Any other electron transfer process lowers the solar cell efficiency.

Dye-sensitized solar technology evolved from the technology of photographic sensitization from metal halide, when Becquerel first observed electricity in two platinum electrodes by dipping them in silver halide solution and exposing to sunlight. ${ }^{16}$ Moser reported that higher currents were produced in silver halide plates when put into a solution containing erythrosine dye. ${ }^{17}$ In 1873 Vogel accidentally discovered that contamination of a silver halide photographic emulsion by a green dye made the film much more sensitive to red light. ${ }^{18}$ Later, Abney succeeded in producing electrical energy using the entire optical solar spectrum. ${ }^{18} \mathrm{~A}$ century later, after the discovery of water splitting by Honda using a wide band gap semiconductor $\left(\mathrm{TiO}_{2}\right)$, scientists started to use the sensitization of semiconductors to convert solar energy into electricity. ${ }^{19}$ However, the efficiency was very low $(<0.5 \%)$ with poor long-term stability. The planer bulk surfaces of $\mathrm{TiO}_{2}$ had very low dye-loading with weak interactions, leading to the low efficiency and weak stability. In 1976 Tsubomura published a paper in Nature reporting a DSSC with an efficiency of about $1.5 \%$ using highly porous multi-crystalline $\mathrm{ZnO}$ powder, $\mathrm{I}^{-} / \mathrm{I}_{3}{ }^{-}$was the redox shuttle and rose Bengal was the dye molecule used to sensitize $\mathrm{ZnO}{ }^{20}$ Fifteen years later, a major breakthrough in DSSC technology was realized when O'Regan and Grätzel published a seminal paper showing a large improvement in efficiency $(>7.00 \%)$ by changing to a $\mathrm{TiO}_{2}$ nanoparticle film platform instead of planar $\mathrm{TiO}_{2}$ and used a Ru-based organometallic dye with the same $\mathrm{I}^{-} / \mathrm{I}_{3}{ }^{-}$redox shuttle electrolyte. To acknowledge this major contribution, DSSCs are now also called Grätzel cells. ${ }^{21}$ 
1.3.2 Fluorine Doped Tin-oxide Substrate and Mesoporous $\mathrm{TiO}_{2} \mathrm{Semiconductor}$

Fluorine doped tin-oxide glass substrates (FTO) are used worldwide due to their low sheet resistance $\left(8-10 \Omega / \mathrm{cm}^{2}\right)$, stability towards humidity, and high temperature stability (> $\left.500{ }^{\circ} \mathrm{C}\right)$. Indium tin-oxide and polymer based conductive plastics showed weak thermal stability. ${ }^{22}$ In low temperature process DSSCs, a polymer substrate can be used as a flexible substrate, but the efficiency is usually very low. Several wide-bandgap oxide semiconductors have been used as photoanodes, such as $\mathrm{ZnO}, \mathrm{SnO}_{2}, \mathrm{SrO}_{3}$, and $\mathrm{Nb}_{2} \mathrm{O}_{5}{ }^{23}$ $\mathrm{TiO}_{2}$ is so far the most desirable for use in DSSCs due to its chemical stability, strong electronic coupling with dye, good optical transmittance in the visible region, resistance to ambient contaminants, abundance in nature, and low cost. Figure 1.3 shows the molecular

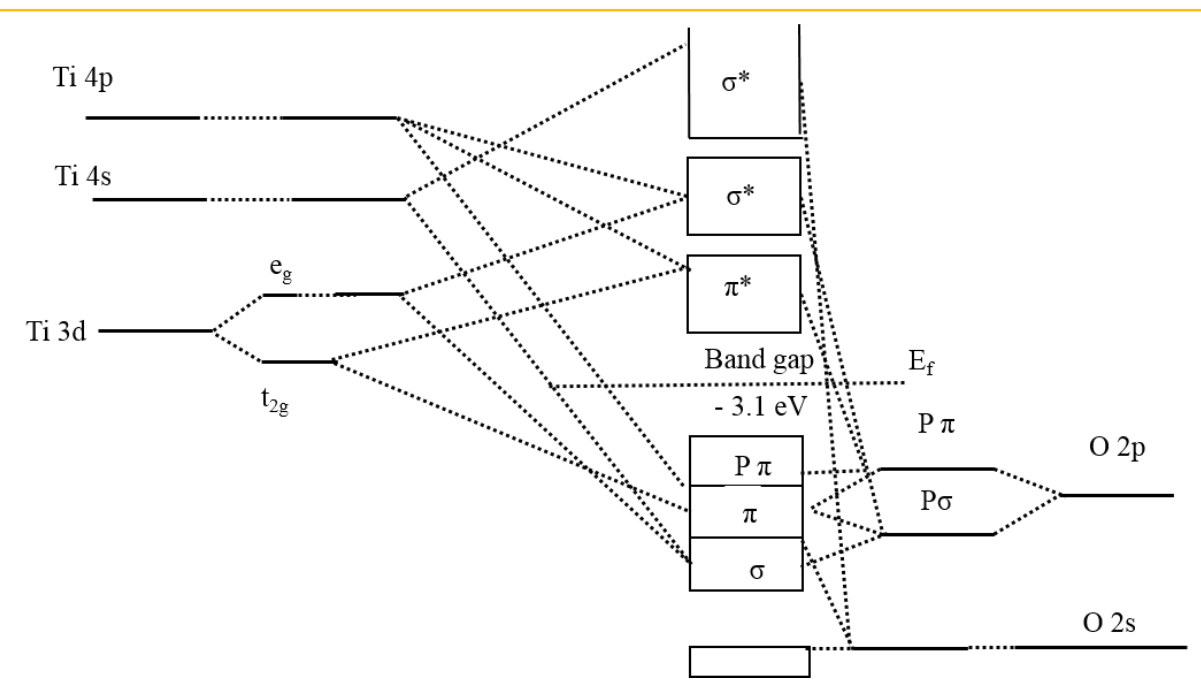

Figure 1.3. Molecular orbital diagram of $\mathrm{TiO}_{2}$.

orbital diagram and band gap of $\mathrm{TiO}_{2}$.

\subsubsection{Dye}

Dye is a key component of a DSSC that can harvest solar light and help convert it into electrical energy. The dye/semiconductor offers three main advantages over pn junction 
diode solar cells. ${ }^{22}$ First, light harvesting can bring charge separation in the DSSC and the separated charges are transported through a semiconductor that hosts the dye. In pn junction solar cells, both processes, charge separation and charge transport, occur in the same medium. Second, there is no electric field setup in a DSSC (transport of charge spontaneously occurs through diffusion), whereas in pn junction solar cells, a built-in electric field is necessary for charge separation and transport. Third, since separated charges in a DSSC move in different media, a temporal phenomenon can exist that controls the charge recombination and regeneration. Excited electrons from a dye choose $\mathrm{TiO}_{2}$ for transportation to the external circuit, and holes choose electrolyte for regeneration of dye. This makes loss of charge generation due to recombination less sensitive to the purity of the substance. However, in a pn junction solar cell where minority and majority charges coexist, even a trace of impurities in the junction can affect the charge transport phenomena. Therefore, high purity substances are absolute requirement to make pn junction solar cells. ${ }^{24}$ This is one of the reasons why DSSC technology has the potential to trim down the cost of fabrication in solar cell technology.

A dye used in DSSC technology should have the following properties. It should have a wide panchromatic capacity to harvest light to produce a large current, high molar extinction coefficient to excite as many electrons as possible, molecular orbitals that match well with the host material conduction band for efficient charge injection, good chemical properties to make a stable monolayer on the host material, and a low HOMO energy level that can be regenerated with electrolyte and produce a large driving force for electron flow through the external circuit. ${ }^{8}$ Inorganic and organic dyes have been developed for increasing panchromatic capacity. Organic dyes have large absorption coefficients due to 
intramolecular $\pi-\pi^{*}$ transitions. ${ }^{25}$ In addition, organic dyes have a wide variety of structures which can provide greater flexibility to modify structures to increase the absorption coefficients. However, the performance of DSSCs based on organic dyes have not yet exceeded those based on inorganic dyes. The other main drawback of organic dyes is their low-term stability. ${ }^{26}$ Inorganic dyes, mainly Ru based metal complexes, have shown the best photovoltaic properties including a high panchromatic capacity, suitable excited and ground state energy levels, relatively long excited-state life-time, and good electrochemical stability. ${ }^{27}$ Among Ru based dyes, N3 and N719 are considered as reference dyes for DSSCs. N719 has greater chemical stability than that of N3. Porphyrin dyes also produce high efficiency DSSCs (12-13\%), but the stability of those dyes is lower than Ru dyes. ${ }^{14}$ Figure 1.4 shows some of the current dyes used in DSSC technology. Figure 1.5 shows how metal

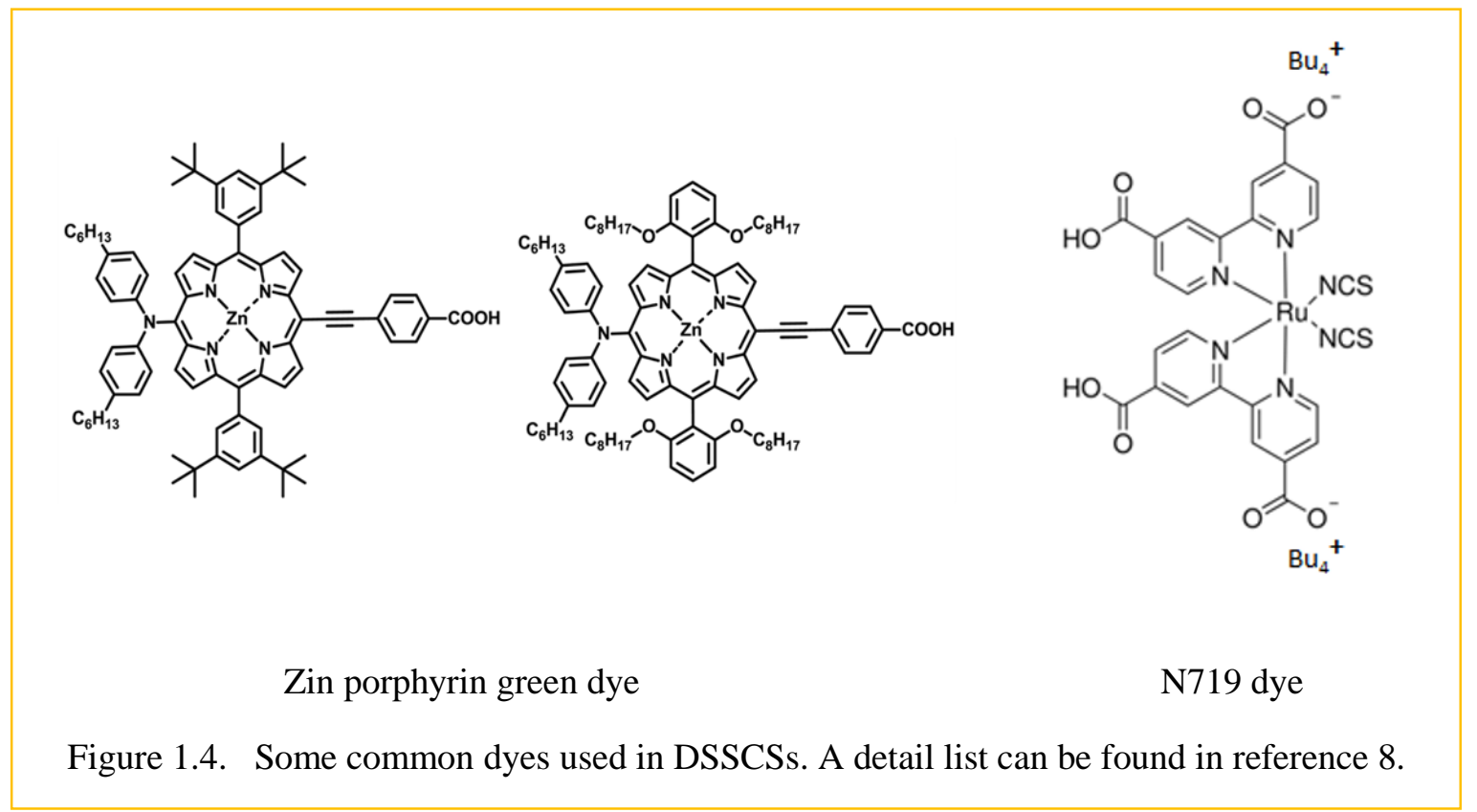

to ligand charge transfer (MLCT) is possible in the N719 dye. In this research, N719 dye was used to make various photoanodes to study the stability and efficiency of our DSSC devices. 


\subsubsection{Electrolyte}

An electrolyte can transport holes in a DSSC to regenerate the dye. Redox couples as an electrolyte used in DSSCs have been copper complexes, pseudohalogen redox couples $\left(\mathrm{SeCN}^{-} /(\mathrm{SeCN})_{3}{ }^{-},\left(\mathrm{SCN}^{-} / \mathrm{SCN}\right)_{3}{ }^{-}\right), \mathrm{Br}^{-} / \mathrm{Br}_{3},{ }^{-}$Cobalt (II, III), and ferrocene-based electrolyte

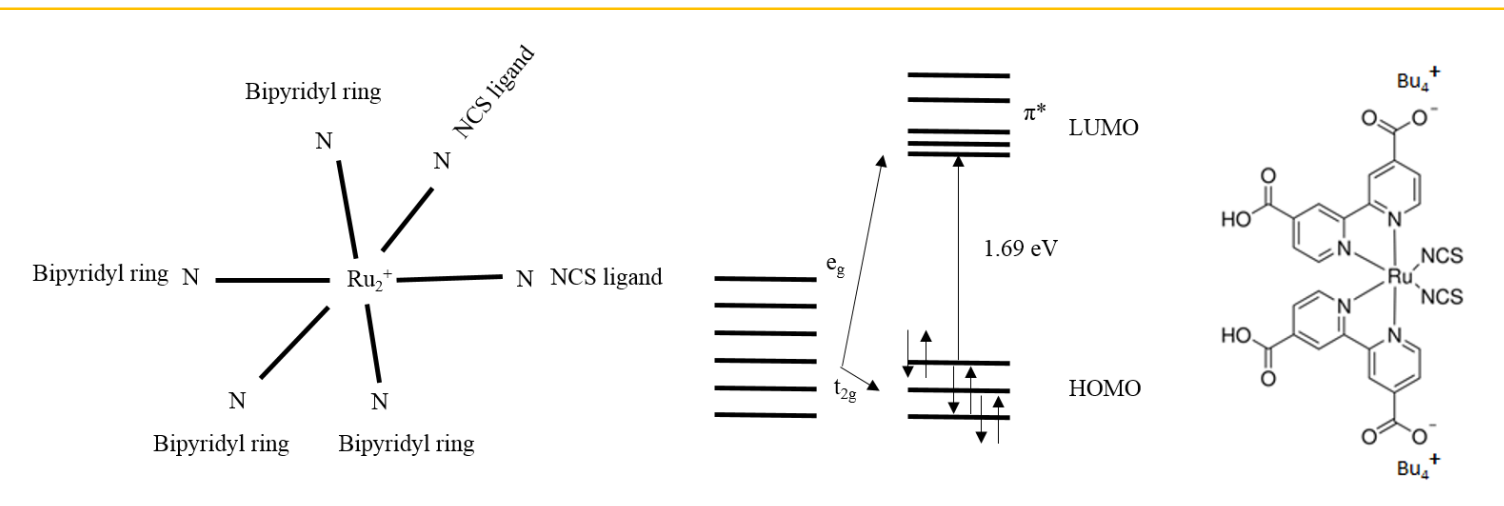

Figure 1.5. Molecular sketch of N719 and metal to ligand charge transfer (MLCT) mechanism. Real molecular sketch is on the left.

$\left(\mathrm{Fc} / \mathrm{Fc}^{+}\right) .{ }^{22}$ Cobalt based electrolytes and porphyrin dyes gave $13.2 \%$ efficient DSSCs. To date, the best electrolyte is the organic solvent-based liquid containing $\mathrm{I}^{-} / \mathrm{I}_{3}{ }^{-}$as a redox couple for the $\mathrm{N} 719 / \mathrm{TiO}_{2}$ combination. In this research, $\mathrm{I}^{-} / \mathrm{I}_{3}{ }^{-}$electrolyte was used to make our DSSC devices. $\mathrm{A} \mathrm{K}^{+} / \mathrm{SCN}^{-}$electrolyte was used to study charge transport dynamics in various electrodes without dye.

\subsubsection{Counter Electrode}

Platinum has been used as a counter electrode material in addition to carbon ${ }^{28}$ (carbon powder and carbon nanotube), cobalt sulfide and conductive polymers. An FTO electrode coated with Pt NPs showed weak long-term stability due to degradation of Pt NPs and diffusion into the $\mathrm{TiO}_{2}$ photoanode, however $\mathrm{CoS}^{26}$ has been identified as a suitable catalyst for the iodide/triiodide redox couple, appropriate for flexible substrates, and eco- 
friendly in comparison to Pt coated FTO, but the stability of the CoS has not been well studied yet. ${ }^{29}$ In this study, Pt coated FTO was used as a counter electrode in all cases.

\subsection{Photoelectrochemical Parameters}

After construction of a solar cell, it is necessary to measure the photoelectrochemical (PEC) parameters to characterize the cell performance. The following sections define the different PEC parameters, including short-circuit photocurrent density $\left(\mathrm{J}_{\mathrm{sc}}\right)$, open circuit photovoltage $\left(\mathrm{V}_{\mathrm{oc}}\right)$, fill factor $(\mathrm{FF})$, and efficiency in percentage $(\eta \%)$, also called power conversion efficiency (PCE).

\subsubsection{Photocurrent Density}

The total current produced by a solar cell per photoanode area upon illumination is called the photocurrent density. In the case of DSSCs, the photocurrent density is governed by the total charge injection from dye to $\mathrm{TiO}_{2}$ and dark current of the devices. A photocurrent in a device can be expressed as:

$$
\begin{gathered}
\mathrm{J}=\mathrm{J}_{\text {inj }}+\mathrm{J}_{\text {dark }} \\
\mathrm{J}_{\text {dark }}=\mathrm{J}_{\text {int }}+\mathrm{J}_{\text {rec }}
\end{gathered}
$$

where $\mathrm{J}_{\mathrm{inj}}$ is the total current due to the injected of electrons from dye to $\mathrm{TiO}_{2}$ and $\mathrm{J}_{\text {dark }}$ is the opposite current developed in a device by the recapture of electrons by electrolyte and dye. $^{30}$ The recapture occurs by two processes, interaction with sensitizer $\left(\mathbf{J}_{\text {int }}\right)$ and recombination of $\mathrm{TiO}_{2}$ electrons $\left(\mathrm{J}_{\text {rec }}\right)$. Short-circuit current density $\left(\mathrm{J}_{\mathrm{sc}}\right)$ is the total 
photocurrent produced by a solar cell of illuminated area of $1 \mathrm{~cm}^{2}$ under sun light at a bias of $0 \mathrm{~V}$.

\subsubsection{Photovoltage}

Photovoltage is the driving force that exists between the quasi-Fermi level $\left(\mathrm{qE}_{\mathrm{F}}\right)$ of the semiconductor and the Nernst potential of the redox electrolyte. The maximum voltage produced from a device at open circuit, where the current is zero, is called the open-circuit voltage $\left(\mathrm{V}_{\mathrm{oc}}\right)$.

\subsubsection{Fill Factor}

FF is defined as the ratio of the maximum electrical power $\left(\mathrm{P}_{\max }\right)$ that can be drawn from the device to the product of $\mathrm{J}_{\mathrm{sc}}$ and $\mathrm{V}_{\mathrm{oc}}$. $\mathrm{P}_{\max }$ is a point in a $\mathrm{J}-\mathrm{V}$ curve where the product of photocurrent $(\mathrm{J})$ and photovoltage $(\mathrm{V})$ is maximum. Mathematically, FF can be expressed as shown below,

$$
\mathrm{FF}=\frac{\mathrm{J}_{\max } \mathrm{V}_{\text {max }}}{\mathrm{J}_{\mathrm{sc}} \mathrm{V}_{\mathrm{oc}}}=\frac{\mathrm{P}_{\text {max }}}{\mathrm{P}_{\mathrm{T}}}
$$

where $\mathrm{J}_{\max }, \mathrm{V}_{\max }$ are the values of $\mathrm{J}$ and $\mathrm{V}$ at the point in the $\mathrm{J}-\mathrm{V}$ curve where their product becomes maximum, and $\mathrm{P}_{\mathrm{T}}$ is the theoretical power of a device. The FF determines the quality of a device. Theoretically, a maximum of $85 \%$ or $0.85 \mathrm{FF}$ can be obtained from a DSSC, and 0.75 is typical for the $\mathrm{TiO}_{2} / \mathrm{N} 719$ dye system. ${ }^{10}$ 


\subsubsection{Efficiency}

The efficiency of a solar cell device is defined as the ratio of the maximum power drawn from the device to the incident power of 1 sun $\left(100 \mathrm{~mW} / \mathrm{cm}^{2}\right.$, solar intensity) illuminated on it. It can be expressed as

$$
\% \eta=\frac{J_{\max } V_{\text {max }}}{P_{\text {in }}}
$$

where $J_{\max }$ and $V_{\max }$ are the values where product of $J \times V$ is maximum in a device, and $P_{\text {in }}$ is the incident solar light of 1 sun. In terms of FF, the efficiency can be expressed as:

$$
\% \eta=\frac{\mathrm{J}_{\mathrm{sc}} \mathrm{V}_{\mathrm{oc}} \mathrm{FF}}{\mathrm{P}_{\text {in }}} \times 100 \%
$$

Although the maximum projected efficiency for DSSCs is $>20 \%$, a maximum efficiency of $13.2 \%^{14}$ has been reported for a porphyrin dye. For $\mathrm{TiO}_{2} / \mathrm{N} 719$ dye, an efficiency of $11.04 \%^{31}$ has been reported. Based on these facts, it can be said that the efficiency of a DSSC can still be improved. Figure 1.6A shows a solar battery that can produce a current upon illumination with sun light. Figure 1.6B describes an I-V curve produced by a solar cell. In general, the current produced by a solar device per square area $(\mathrm{J})$ is plotted against the voltage applied to the device in opposite direction. When the applied voltage is zero, the total current will be due to the solar light only, and is denoted by a short circuit current $\left(\mathrm{J}_{\mathrm{sc}}\right)$. This is the maximum possible current that a solar device can produce per unit area. When the applied voltage is equal to a device potential, photocurrent stops flowing and the voltage developed in the device is known as open circuit voltage $\left(\mathrm{V}_{\mathrm{oc}}\right)$. $\mathrm{V}_{\mathrm{oc}}$ is the maximum possible voltage that a solar device can produce. A product of $\mathrm{J}_{\mathrm{sc}}$ and $\mathrm{V}_{\mathrm{oc}}$ is the total power developed 
in a solar cell due to sun light, and it is the theoretical maximum power $\left(\mathrm{P}_{\mathrm{T}}\right)$ that a solar cell possesses. However, there is always a loss in power because of internal resistance due to electrical contact. A maximum power that a solar device can produce for external work is known as $\mathrm{P}_{\max }$ or $\mathrm{P}_{\text {out }}$ and is equal to the product of maximum current $\left(\mathrm{J}_{\max }\right)$ and maximum voltage $\left(\mathrm{V}_{\max }\right)$. Total solar light intensity falling on an active area of a solar device is input

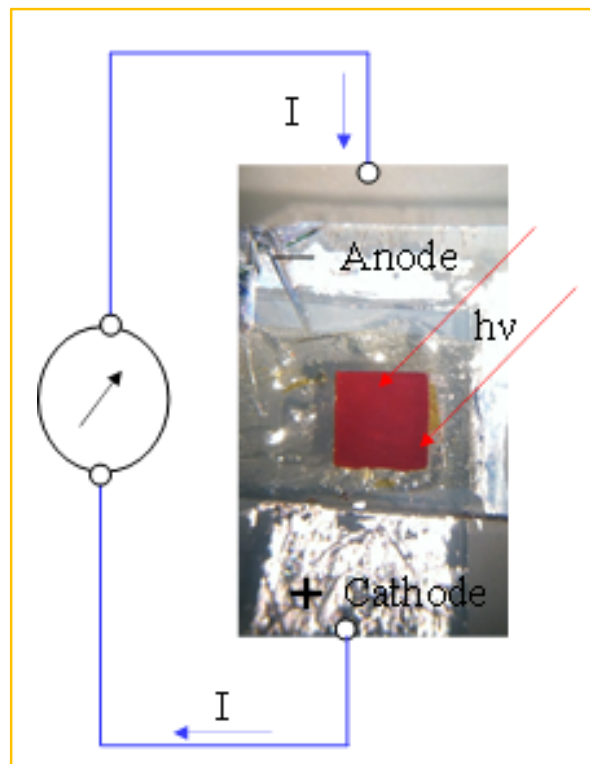

Figure 1.6A

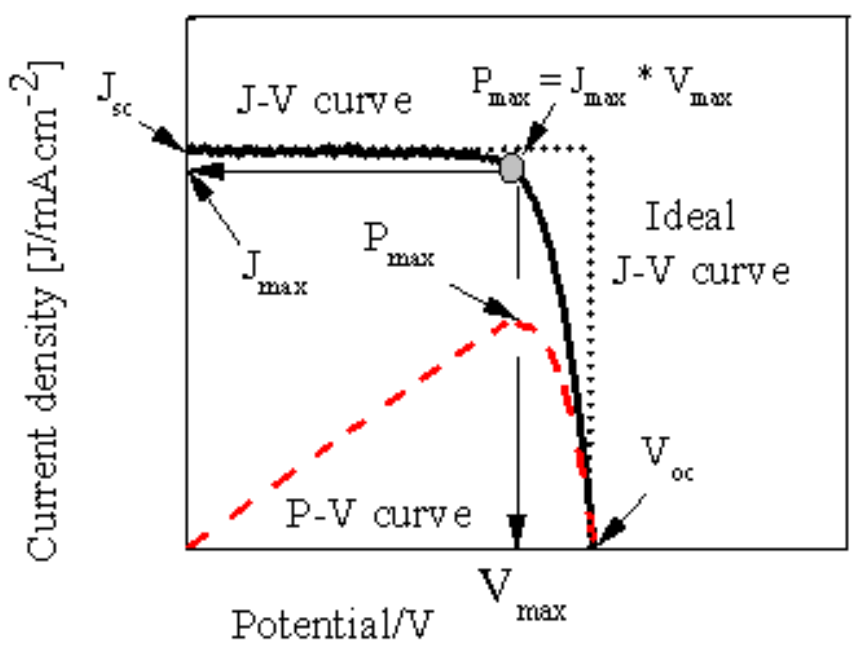

Figure 1.6B

Figure 1.6 A solar cell battery (A), J-V curve produced by a device at operating condition (B).

energy, $P_{\text {in }}$ in $\mathrm{mW} / \mathrm{cm}^{2}$. Solar intensity falling on the earth varies from place to place, morning to evening, and throughout a year. In addition, earth's atmosphere absorbs solar radiation. Considering all these factors, a standard of solar intensity is calculated for measuring an efficiency of a solar cell, which is the average amount of solar intensity arrived on the sea surface per unit area after traveling the mass of air equal to 1.5 times the mass of air measured directly overhead. The value 1.5 A.M. solar intensity is $100 \mathrm{~mW} / \mathrm{cm}^{2}$ and is also known as 1 sun. Efficiency in percentage of a device is calculated by multiplying a 
ratio of $\mathrm{P}_{\max }$ and $\mathrm{P}_{\text {in }}$ by 100 . When $\mathrm{J}_{\max }$ is expressed in $\mathrm{mA}, \mathrm{V}_{\max }$ in $\mathrm{V}$, and one sun in $\mathrm{mW}$, efficiency $\%$ of a device will be simply $\mathrm{P}_{\max }$.

\subsection{Motivation of the Study}

The current state of technology, which is being enjoyed by human society, is not possible without the consumption of easily available energy resources such as fossil fuels, hydroelectricity, and chemical energy from batteries. A huge demand and consumption of energy occurred after the industrial revolution. This not only led to the 1972 energy crisis, but also severe environmental issues, such as global warming, resulting from the large production of greenhouse gases as the products of fossil fuels. As a result, eco-friendly renewable energy resources are needed as a practical solution to address the future energy needs and environmental concerns. Among the many forms of renewable energy, the most practical solution is solar energy. Robust silicon solar technology is still not feasible mainly because of its high cost of fabrication. Dye-sensitized solar cell technology has emerged as a robust technology with the potential to replace silicon technology due to its low fabrication cost, ability to work in diffuse light, and its ability to produce flexible devices that can be applicable everywhere. However, it has several challenges that have still not been addressed completely. The main challenges are optimization of efficiency to beyond $20 \%$ for commercialization, production of chemically stable photoanodes for up to 20 years, and the more complete understanding of charge transfer dynamics within DSSCs, especially at the dye/semiconductor interfaces. The motivation of this work was to focus on the dye/semiconductor interface in order to solve these challenges. Specially, we applied surface chemistry to functionalize the semiconductor surface for the production of more stable devices and create materials with improved efficiency. We addressed the limitations of 
chemical stability existing between the $\mathrm{TiO}_{2} / \mathrm{N} 719$ dye system by preparing more stable structures through covalent attachment of dye to the semiconductor surface. We provided a strong attachment of dye on the semiconductor surface and studied the charge transfer dynamics using ultrafast laser technology. We also incorporated $\mathrm{Nd}_{2} \mathrm{O}_{3}$ into the $\mathrm{TiO}_{2}$ films, which resulted in enhanced efficiency. Finally, we improved the efficiency of DSSCs by chemical attachment of plasmonic nanostructures to the photoanodes.

\subsection{Outline of the Dissertation}

Chapter 2 of this dissertation discusses the methodological approaches for preparing high efficiency DSSC devices. A new approach for sealing devices using a thermal press along with a full discussion of the measuring system will be discussed in Chapter 2. Chapter 3 describes how to prepare stable devices while maintaining high efficiency by covalent modification of N719 dye to the photoanodes. An amide bond created between $\mathrm{N} 719$ and chemically modified $\mathrm{TiO}_{2}$ with APTES led to more stable dye. Possible mechanisms of degradation of the devices with and without modification when stored in air are described. The SCN ligand on the dye is preserved in the stable devices, which is also discussed. Chapter 4 describes the charge transfer dynamics between $\mathrm{N} 719$ dye and $\mathrm{TiO}_{2}$ prepared by various methods, including two different covalent linkers and direct adsorption. The effect of multilayer adsorption of dye and the effect of linear and aromatic linkers on the charge transfer dynamics is described. These findings may lead to improvements in the PCE of devices. A dramatic impact of doping rare earth oxides, especially micro-sized $\mathrm{Nd}_{2} \mathrm{O}_{3}$ particles, into $\mathrm{TiO}_{2}$ NPs to enhance the PCE is described in Chapter 5. Finally, the

performance of DSSCs employing surface modification of $\mathrm{TiO}_{2}$ with plasmonic Au NPs by 
electrostatic attachment is described in Chapter 6. Reasons for improvement in the PEC parameters will be discussed as determined by electrochemical and spectroscopic measurements. 


\section{CHAPTER 2}

\section{EXPERIMENTAL SECTION}

The preparation of DSSCs is crucial for the advancement of DSSC technology. Every component of DSSCs can influence the PEC parameters. Even a little variation in electrolyte, for example, can reduce photocurrent. The quality of materials, variation of spacer distance between the photoanode and the counter electrode, and quality of electrical contacts on the electrodes can result in different values of short-circuit photocurrents and

photovoltages. Both physical and chemical engineering should be optimized to get high efficiency devices. This chapter describes the methods used to prepare DSSCs in the work later described in this dissertation. It also describes the measurement system adopted and a short description of the pump-probe instrument used for ultrafast transient absorption spectroscopy.

\subsection{Chemicals and Materials}

General cleaning solvents such as ethanol, 2-propanol, and acetone were bought from Sigma-Aldrich, USA. Nanoparticle binder (ethyl cellulose) and nanoparticle separator (terpineol) were used to prevent aggregation of nanoparticles. Degussa (P25), DSL-18 nm, DSL-30NRD, Solaronix T/SP-20 nm, and R/SP (scattering particles of titania, 200-400 nm) were used to conduct this research. Degussa $\mathrm{TiO}_{2}$ powder was obtained from Sigma-Aldrich. 
DSL-18 nm and DSL-30NRD were purchased from Dyesol, Australia. T/SP was purchased from Solaronix, Switzerland. Scattering layer particles (WRS-200 and R/SP) were obtained from Dyesol and Solaronix, respectively. The aromatic molecular linker, paminophenyltrimethoxysilane was purchased from Gelest, USA. The alkane molecular linker, 3-aminopropyltriethoxysilane was purchased from VWR, USA. Organo-metallic ruthenium dye [(Cis-diisothiocyanato-bis (2, 2'-bipyridyl-4, 4'-dicarboxylato Ru (II) bis (tetrabutylammonium) N719] was purchased from both Dyesol and Solaronix. Fluorine doped tin-oxide transparent glass (FTO) electrodes were purchased from Hartford Glass, Indiana (USA). Pt in solution (platisol) used to prepare the counter electrodes was obtained from Solaronix. For molecular deposition of $\mathrm{TiO}_{2}$ on $\mathrm{FTO}, \mathrm{TiCl}_{4}$ was purchased from both VWR and Sigma-Aldrich. Vacuum sealant (Epoxy) was purchased from Ideal Vacuum Products, USA. For hermetically sealing of devices, $25 \mu \mathrm{m}$ and $60 \mu \mathrm{m}$ thick Surlyn plastic ionomer was obtained from both Solaronix and Dyesol. A thermal press was used in order to properly sandwich the photoanode and counter electrode together with sealant. It was obtained from HeatPress Nation (model number MPPRESS912), USA. In order to obtain images of $\mathrm{TiO}_{2}$ films on the nanoscale, silicon AFM tips were used and were purchased from Nanoscience Instruments (USA) and used with atomic force microscopy (AFM). Hydrogen tetrachloroaurate was synthesized in the laboratory according to literature, ${ }^{32}$ stored in the freezer, and used to synthesis Au NPs. Neodymium oxide mesh powder used to dope $\mathrm{TiO}_{2}$ was purchased from Sigma-Aldrich.

\subsection{Preparation of Titanium Oxide Photoanodes}

P25 powder was used in this research for preliminary studies. The following steps 
as shown in the Scheme 2.1 were used to prepare photoanodes for DSSC samples using titanium oxide nanoparticles. Scheme 2.2 was used to assemble DSSC samples. Five different types of $\mathrm{TiO}_{2}$ nano-paste were used in this study: transparent $\mathrm{TiO}_{2}$ nano-particle,

Scheme 2.1 Preparation of photoanodes for dye-sensitized solar cells.

Preparation of photoanode (PA)

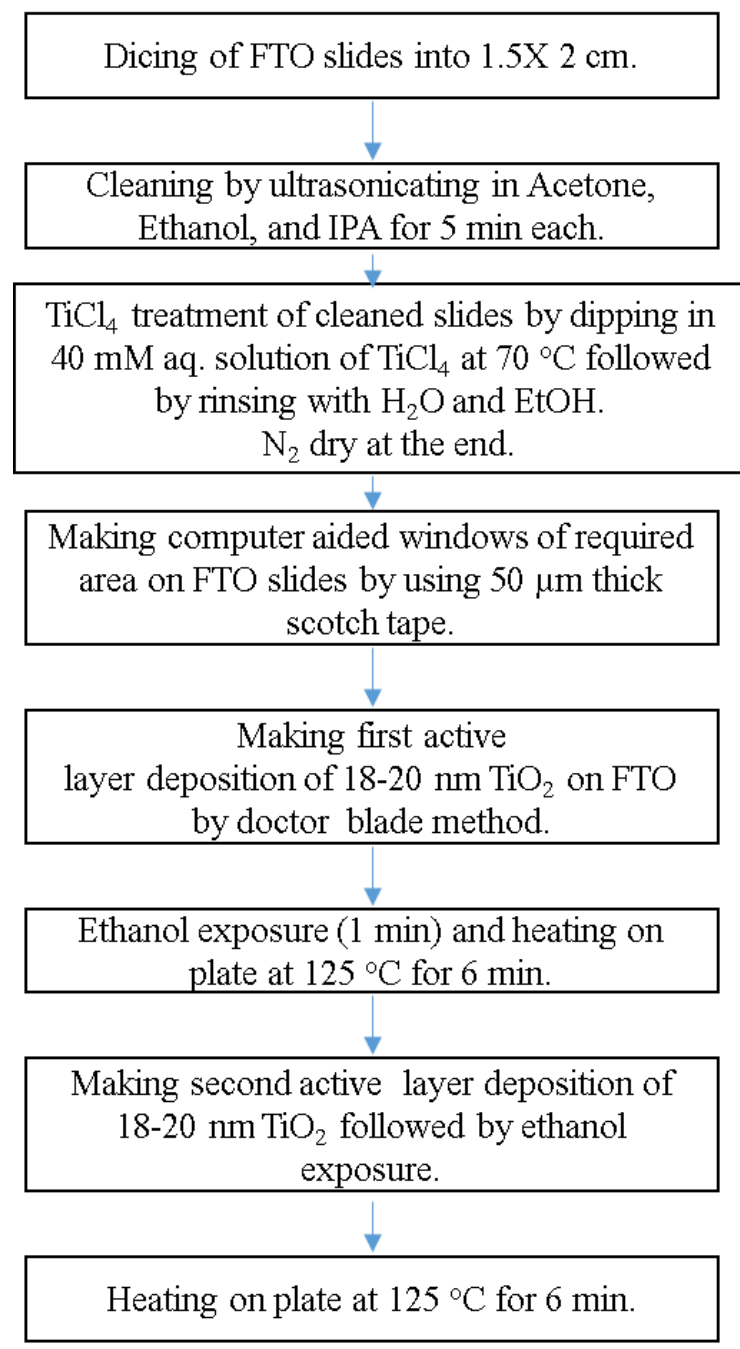

Making single scattering layer deposition of 200-400 $\mathrm{nm} \mathrm{TiO} 2$ on FTO by doctor blade method followed by EtOH exposure.

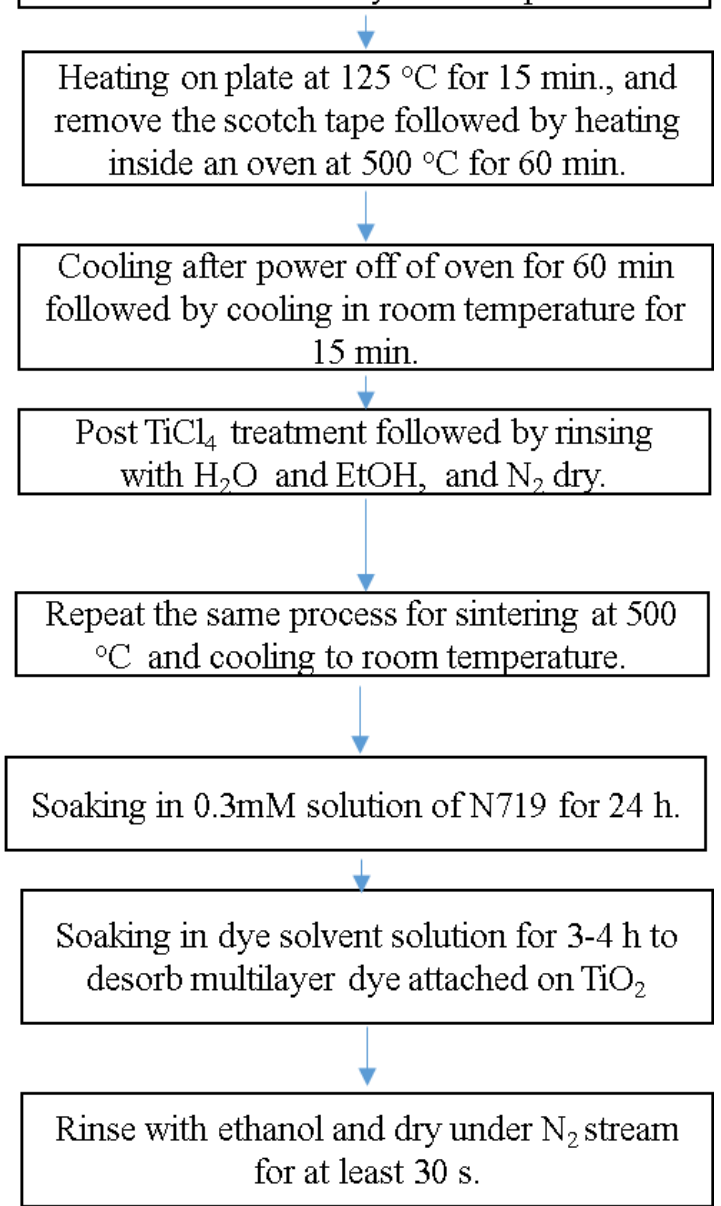

18-20 nm from Dyesol (DSL-18), transparent $\mathrm{TiO}_{2}$ nanoparticles $30 \mathrm{~nm}$ from Dyesol, DSL30NRD, transparent $\mathrm{TiO}_{2}$ nanoparticles 20-25 nm (T/SP) from Solaronix, degussa $\mathrm{P} 25 \mathrm{TiO}_{2}$ (20\%) nanoparticle modified by mixing t-terpineol (70\%), ethyl cellulose (10\%), and degussa $\mathrm{P} 25 \mathrm{TiO}_{2}(20 \%)$ nanoparticle modified by mixing t-terpineol (70\%), ethyl cellulose $(10 \%)$, and acetic acid in ethanol using the reference. ${ }^{33}$ 
Various steps involved in the preparation of photoanodes in the laboratory can be described in Scheme 2.1 and can be described in the pictorial form as follows:

Scheme 2.2. Fabrication of dye-sensitized solar cells.

Preparation of counter electrode (CE)

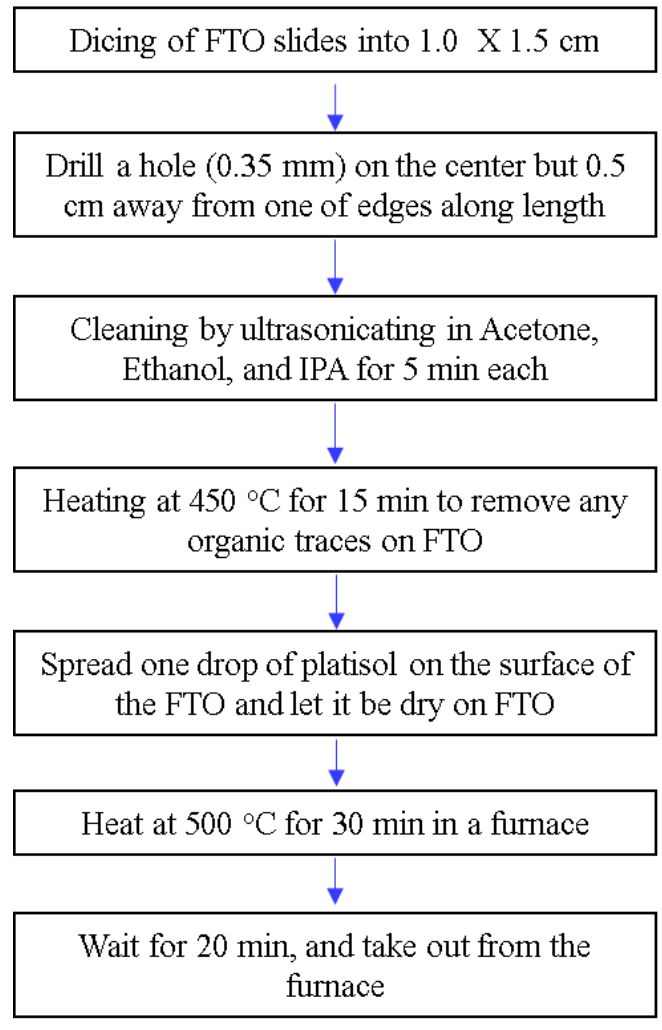

Fabrication of a dye-sensitized solar cells

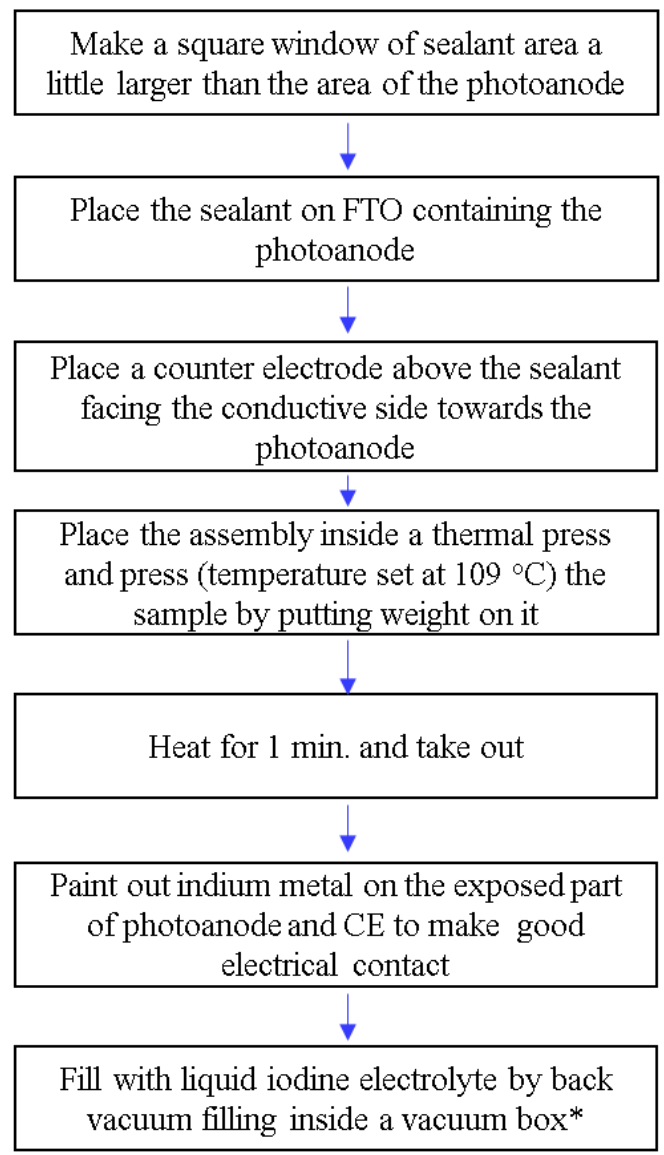

*In back vacuum filling, a drop of electrolyte is cast on top of the hole of the counter electrodes and air is pumped-out. Bubbles of air comes out through the drop. When the drop become free of bubbles, air is pumped-in. Air pushes the electrolyte, and fills up the porous part of the photoanode.

Step 1. Bare FTO can trigger recombination of electrons with electrolyte when electrons are making route to external circuit from $\mathrm{TiO}_{2}$ nanoparticles. To prevent this recombination, a molecular layer of $\mathrm{TiO}_{2}$ itself is deposited on FTO. The molecular layer does not inhibit the conductivity of FTO. FTO slides were cleaned by dipping in detergent solution for 15 min and then sonicated 5 min each time in acetone, ethanol, and IPA. The last solvent IPA 
was used to rinse FTOs and dried under stream of $\mathrm{N}_{2}$ gas. The deposition of the blocking layer formed on FTO occurred by dipping the FTO in a $40 \mathrm{mM}$ aqueous solution of $\mathrm{TiCl}_{4}$ at
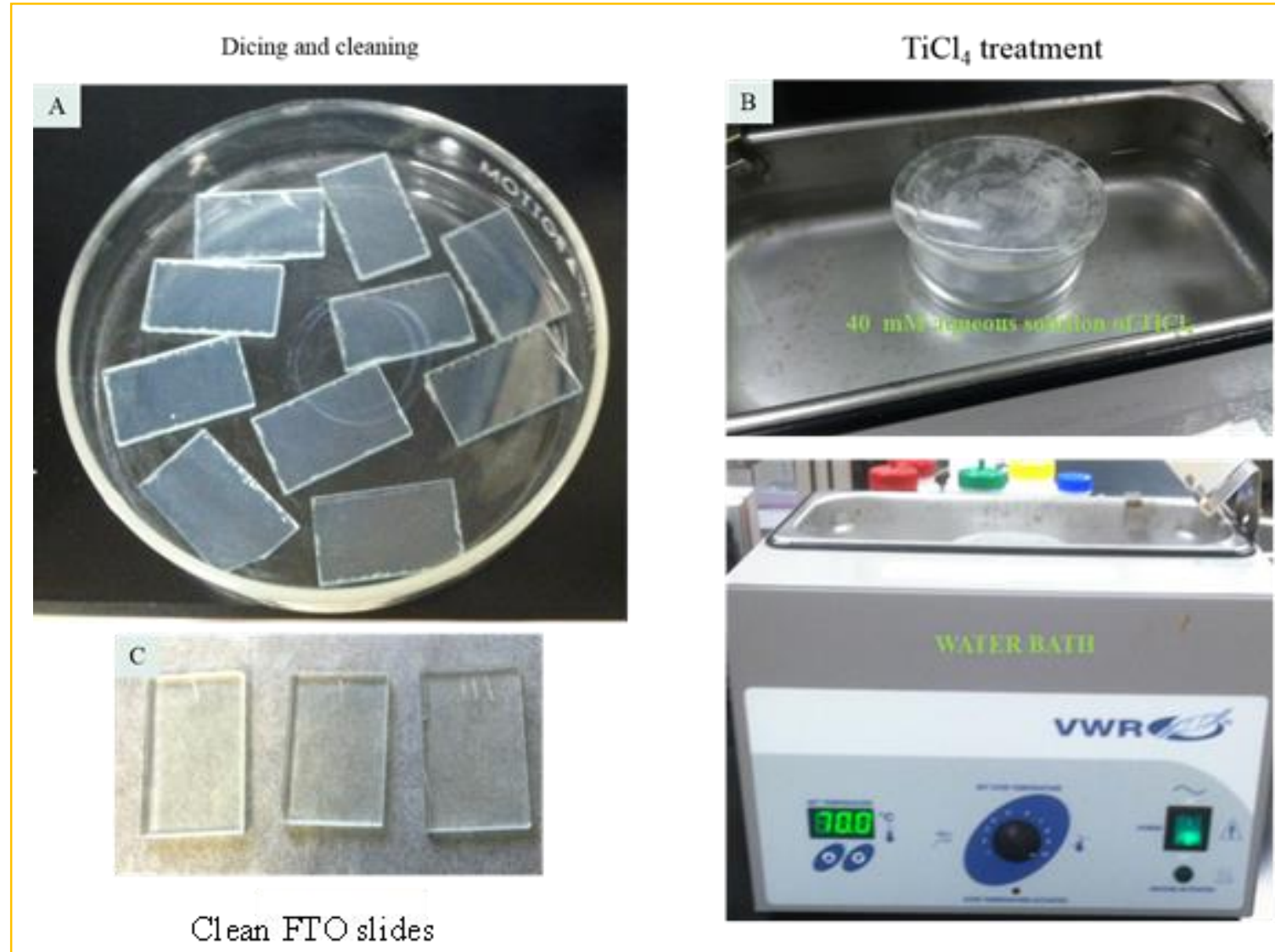

Figure 2.1. Deposition of a $\mathrm{TiO}_{2}$ blocking layer on FTO slides starting from the very beginning stage of preparation, including dicing, cleaning, and $\mathrm{TiCl}_{4}$ treatment.

$70{ }^{\circ} \mathrm{C}$ for $30 \mathrm{~min}$. in a water bath, followed by rinsing with nanopure water first and then pure ethanol. The rinsed samples were dried under a stream of dry $\mathrm{N}_{2}$ for $1 \mathrm{~min}$. The process is shown in Figure 2.1.

Step 2. Next we deposited the active layer $\left(\mathrm{TiO}_{2}\right.$ nanoparticles, $\left.20-35 \mathrm{~nm}\right)$ by the doctor blade method. Scotch tape was used to make a window of desired areas such as $1 \mathrm{~cm}^{2}$ (for UV-vis, and ATR study), $0.3 \mathrm{~cm}^{2}, 0.2 \mathrm{~cm}^{2}(0.4 \mathrm{x} 0.5 \mathrm{~cm})$, and commonly $0.16 \mathrm{~cm}^{2}(0.4 \mathrm{x} 0.4$ $\mathrm{cm})$. Clean and smooth glass slides were employed to make paste on these areas. A small amount of $\mathrm{TiO}_{2}$ paste was placed on one edge of the area on scotch tape as shown in Figure 
2.2. A glass slide slanting at $45^{\circ}$ was used to deposit the paste by pressing a little hard and sliding the paste over the exposed area on the FTO only one time. The deposited paste was

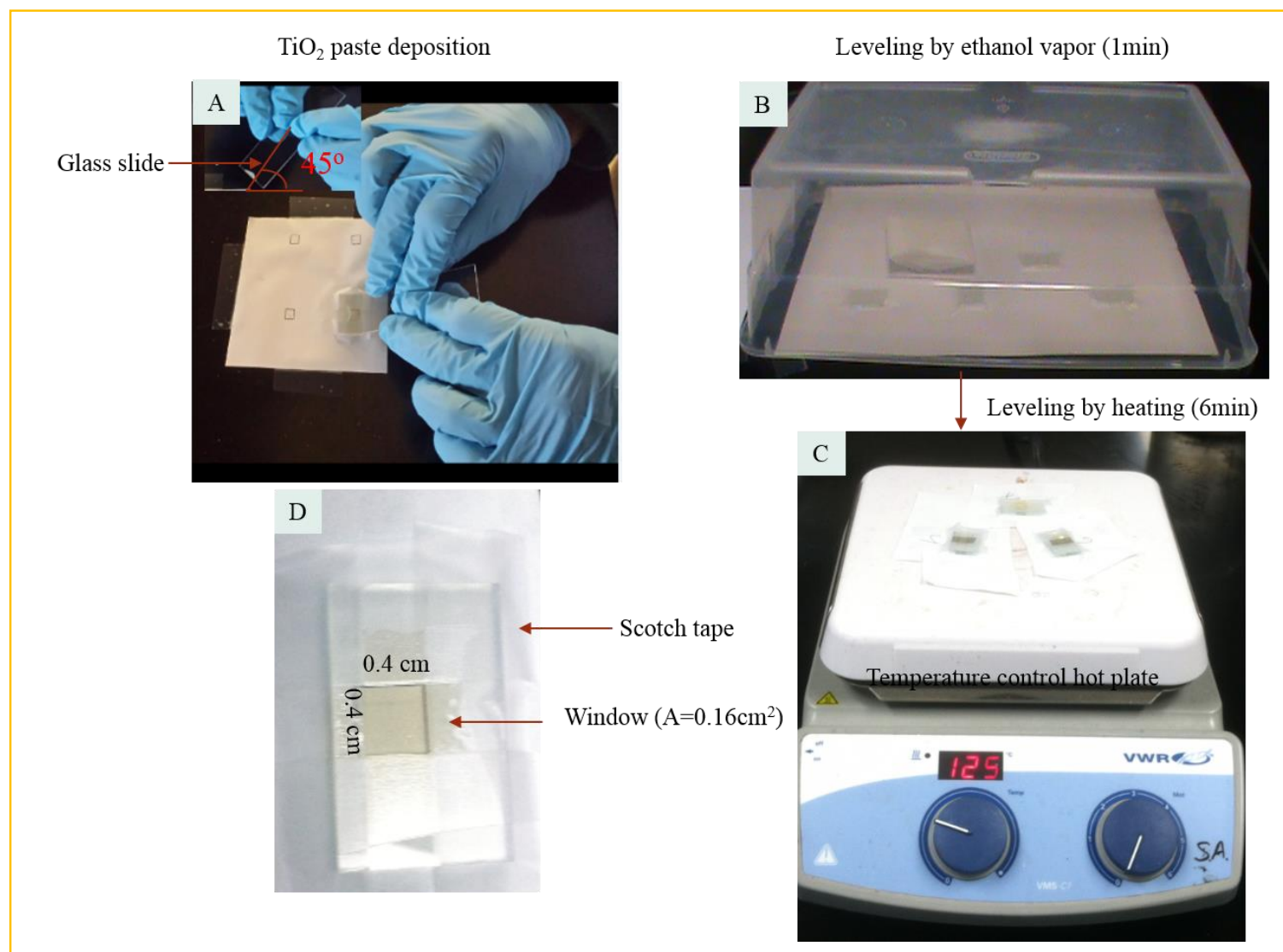

Figure 2.2. Preparation of the $\mathrm{TiO}_{2}$ active layer on FTO by the doctor blade method.

exposed to an environment filled with saturated ethanol vapor inside a closed box for one minute. The ethanol treated paste was placed immediately on a hot plate at $125^{\circ} \mathrm{C}$ for 6 min without peeling off the scotch tape. A second layer of $\mathrm{TiO}_{2}$ paste was deposited following the same method.

Step 3. A single scattering layer of 200-400 $\mathrm{nm} \mathrm{TiO}_{2} \mathrm{NPs}_{\text {sas }}$ deposited on top of the active layer following a similar process, except we used 15 min for leveling time after ethanol exposure, instead of $6 \mathrm{~min}$. Therefore, the process is not repeated here, however, Figure 2.3 shows pictures of the process. The scattering nanoparticles come as a white paste. 
Step 4. The scotch tape was carefully peeled off and the samples were heated in a closed oven at a temperature of $500{ }^{\circ} \mathrm{C}$. The temperature ramp in the oven was $2{ }^{\circ} \mathrm{C}$ per second. After $1 \mathrm{~h}$, the power supply to the oven was turned off. Samples were allowed to cool for 1 h. The door of the oven was then opened. After $15 \mathrm{~min}$, samples were completely taken out
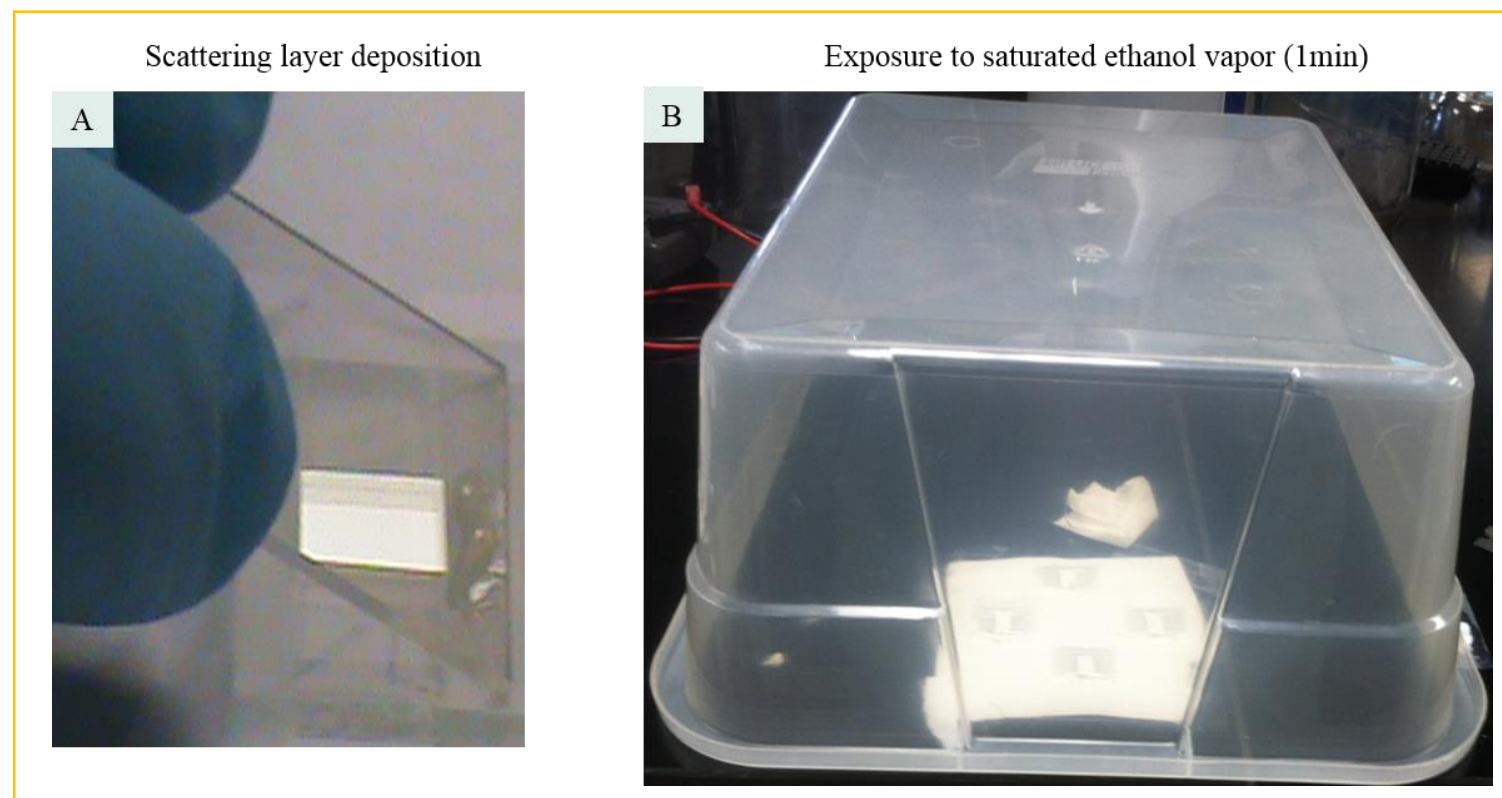

Figure 2.3. Single layer deposition of the scattering layer on top of the active layer.

from the oven and cooled to room temperature. Figure 2.4 shows the sintering process. The top figure shows the PAs placed inside the oven.

Step 5. The sintered samples were post treated with $40 \mathrm{mM} \mathrm{TiCl}_{4}$ again at $70{ }^{\circ} \mathrm{C}$ for 30 min in a water bath followed by rinsing with nanopure water first and then pure ethanol. The rinsed samples were dried under $\mathrm{N}_{2}$ for $1 \mathrm{~min}$. $\mathrm{TiCl}_{4}$ helped to connect $\mathrm{TiO}_{2}$ nanoparticles for better charge transfer. The post treated samples were sintered at $500{ }^{\circ} \mathrm{C}$ for $1 \mathrm{~h}$ and allowed to cool inside the oven for $1 \mathrm{~h}$ with the oven off, followed by $15 \mathrm{~min}$ at room temperature after opening the door of the oven. These samples are known as $\mathrm{FTO} / \mathrm{TiO}_{2}$ electrodes. Figure 2.5 shows the pictures of post-modification and sintering. 
Step 6. A stock solution of dye should be prepared at least 3-4 hours before using in order to dissolve all solid dye into the solvent. The concentration of the dye solution was $0.3 \mathrm{mM}$

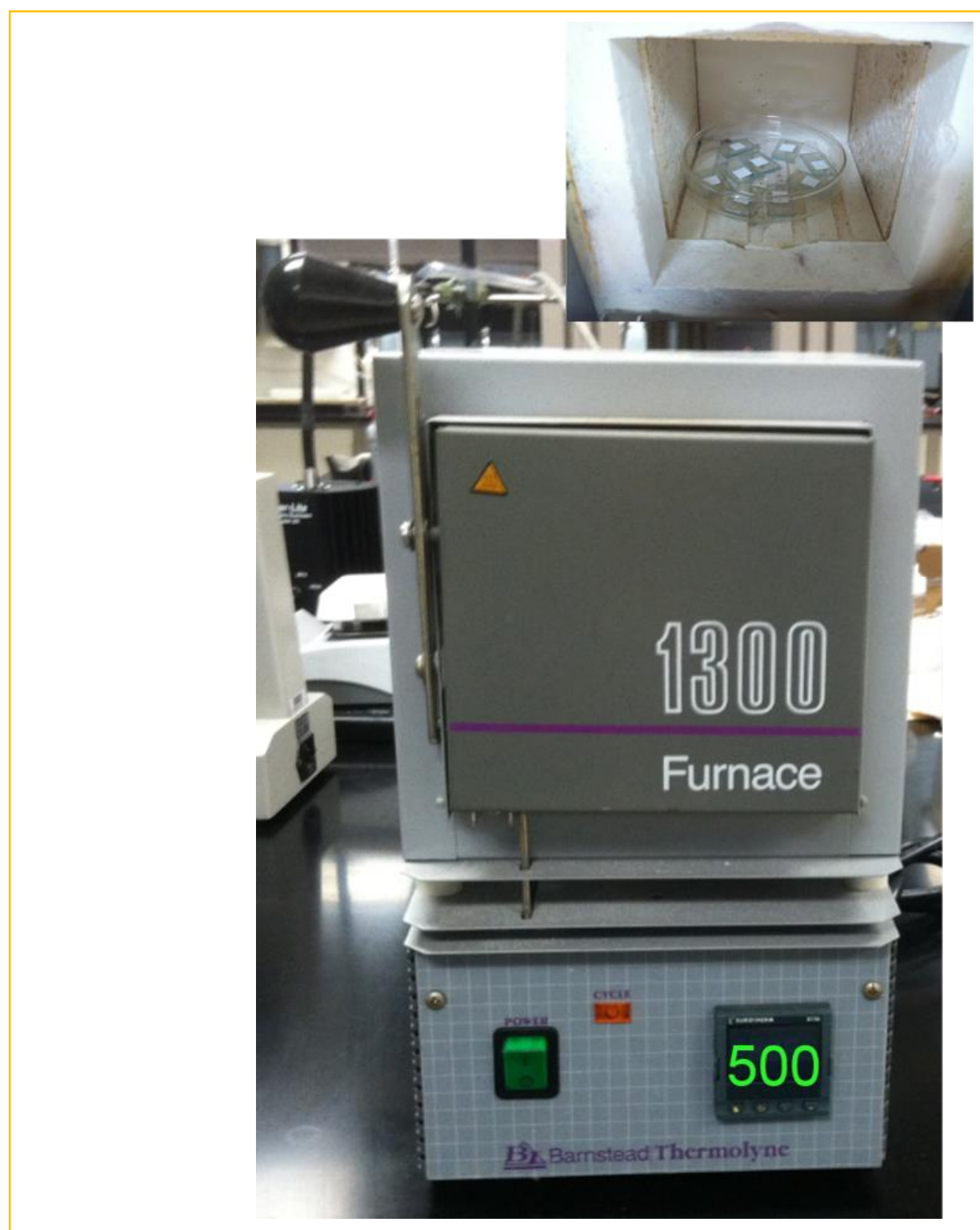

Figure 2.4. Sintering of $\mathrm{TiO}_{2}$ on $\mathrm{FTO}$ at $500{ }^{\circ} \mathrm{C}$. The top figure depicts the inside of the oven containing $\mathrm{FTO} / \mathrm{TiO}_{2}$ electrodes on a crystalizing dish.

usually in a 50:50 mixture of acetonitrile and t-butanol in this case. Different solvents were chosen for covalent bonding and various control experiments. The solution vessel should be wrapped by light insensitive material to store the dye solution for future use. Freshly prepared samples were dipped into the dye solution in a clean crystallizing dish, covered by 
a watch glass, and wrapped with aluminum foil to prevent any light triggered reaction during the sensitization process. Photoanodes remained in the solution for $24 \mathrm{~h}$. After $24 \mathrm{~h}$ the samples were removed and soaked into the same solvent without dye for 3-4 h. This later
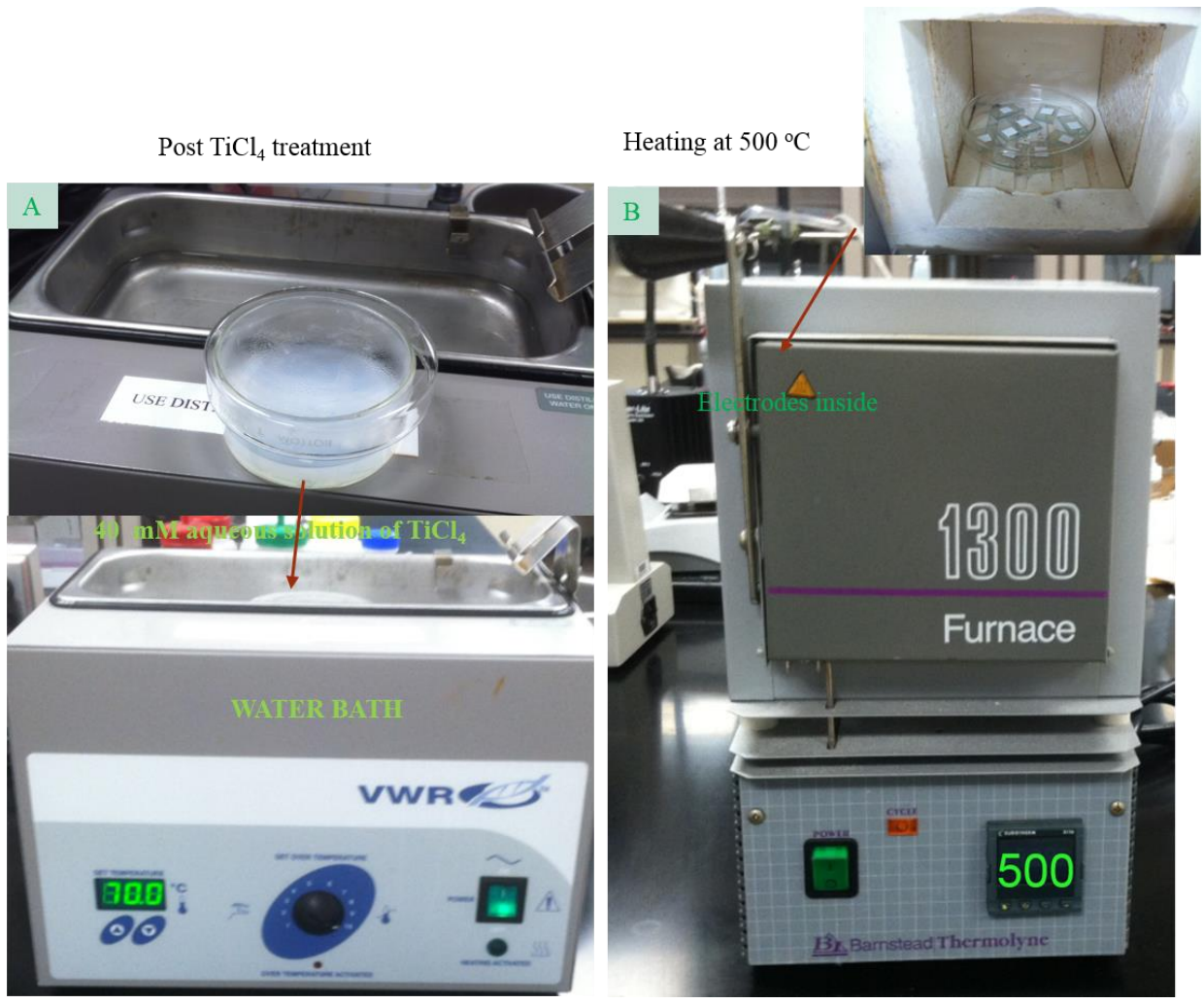

Figure 2.5. Post treatment of $\mathrm{FTO} / \mathrm{TiO}_{2}$ samples Figure (A) and sintering at $500{ }^{\circ} \mathrm{C}$ Figure (B). The top figure on Figure (A) is crystallizing dish with samples dipped into $40 \mathrm{mM} \mathrm{TiCl}_{4}$ aqueous solution. This is actually inside the water bath. The Figure (B) shows the samples sintered at 500 ${ }^{\circ} \mathrm{C}$ inside the oven. The top part of the figure shows inside of the oven where samples were sintered.

process is to desorb any multilayer dye adsorbed on the samples. The samples were rinsed with a gentle current of ethanol for at least 1 min continuously and dried under a gentle stream of $\mathrm{N}_{2}$ gas. This is known as an $\mathrm{FTO} / \mathrm{TiO}_{2} /$ Dye photoanode. The color of the photoanode should be deep-red as shown in Figure 2.6 when observed from the nonconductive side. These samples were sealed immediately with a CE by using surlyn sealant (DuPont) as described in Step 7. 


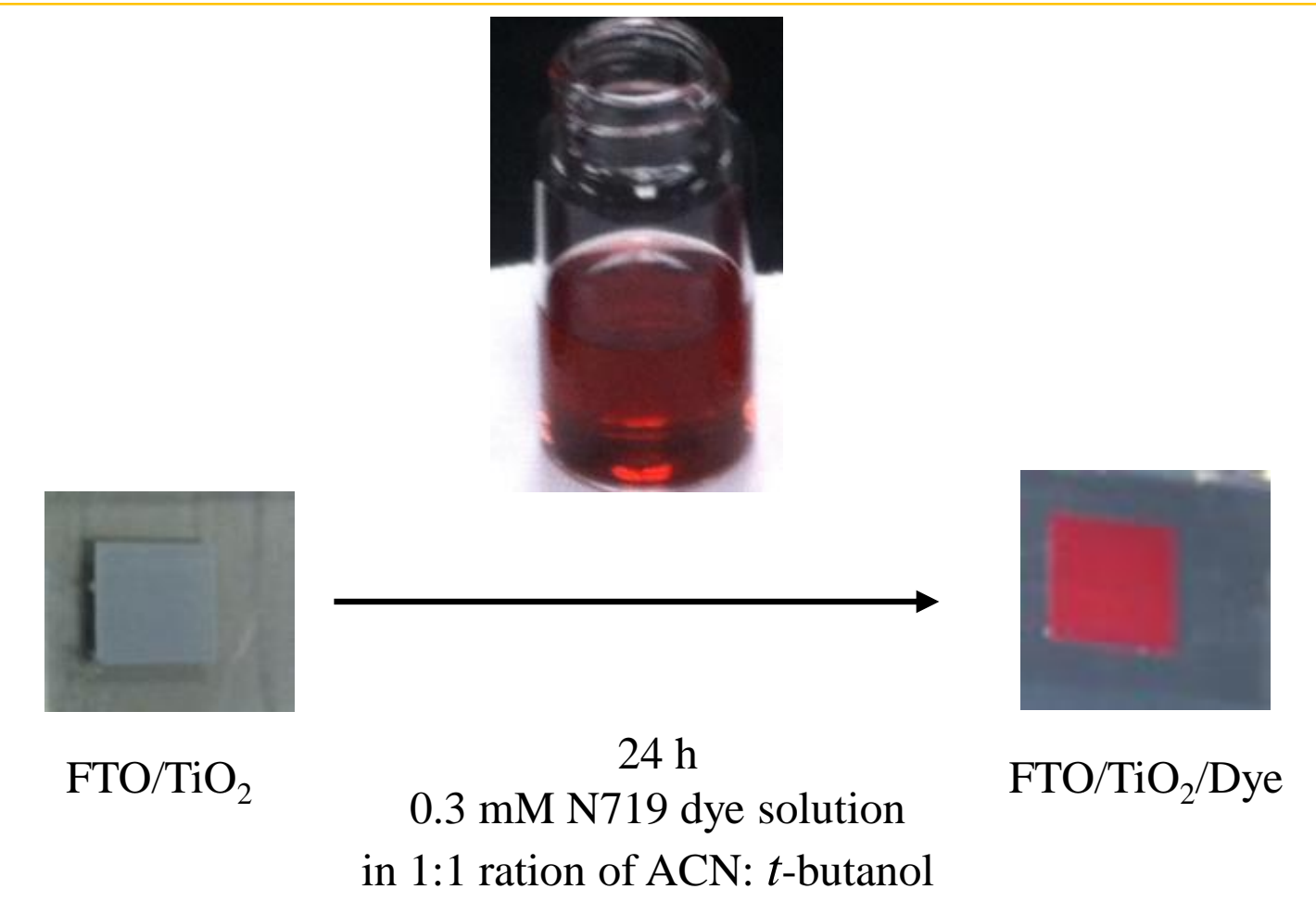

Figure 2.6. Sensitization of $\mathrm{FTO} / \mathrm{TiO}_{2}$ electrodes with N719. Red picture is from non-conductive part. The scattering layer does not absorb dye. For simplicity dye solution in a vial is shown here.

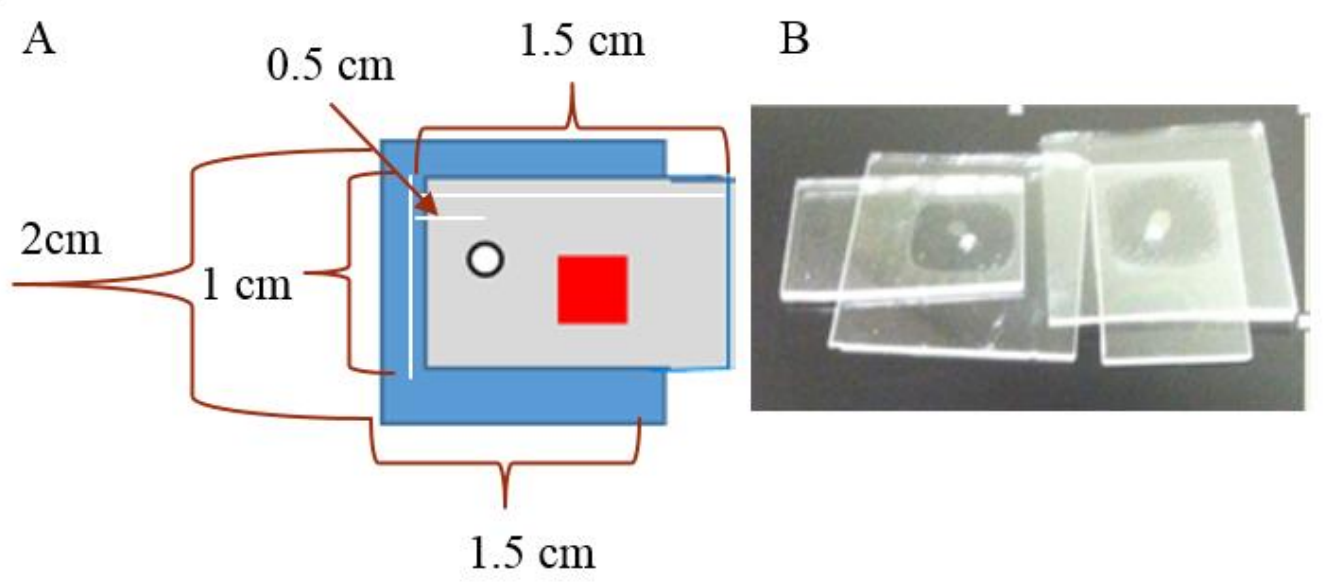

Figure 2.7. Design of the device with a hole on the CE (A), and photograph of the hole and sealing performed on the glass slides (B). 
Step 7. Counter electrodes were prepared during the dye sensitization. A small hole was drilled on an FTO slide from the non-conductive side. A special drilling tip $(0.8 \mathrm{~mm})$ (diamond tip from Ukam, USA) was used. On a 1.0 x $1.5 \mathrm{~cm}$ slide, a hole was made on a spot $0.5 \mathrm{~cm}$ from one of the edges of the lateral side and on the middle of transverse side as illustrated in Figure 2.7. Figure 2.7A shows the device sealed with a counter electrode with a drilled hole. Figure 2.7B is a photograph after the sealing process was performed on glass (not FTOs) slides. The drilled FTO was cleaned in a way as described before in step 1. Before coating with platisol, the clean FTO was heated on a hot plate at $450{ }^{\circ} \mathrm{C}$ for $15 \mathrm{~min}$ to remove any organic residue. A drop of platisol was cast onto the clean FTO and heated inside an oven at $500{ }^{\circ} \mathrm{C}$ for $30 \mathrm{~min}$. The heated counter electrodes were cooled inside an oven for $30 \mathrm{~min}$ and subsequently at room temperature for $30 \mathrm{~min}$.

Step 8. Sandwich cells were prepared by inserting plastic ionomer (Surlyn) between the working electrode and counter electrode and heating at $118{ }^{\circ} \mathrm{C}$ for one minute in a thermal press (model number, MPRESS912, HeatPress Nation, USA). The sample with surlyn was placed at the center of the floor of the thermal press as shown in Figure $\mathbf{2 . 8}$ and pressed by hand for 1 minute (caution: the FTO glass should not break). For more improvement in hermetical sealing of devices, epoxy was placed all around the contact joining the photoanode and the counter electrode and heated at $60{ }^{\circ} \mathrm{C}$ for $1 \mathrm{~h}$ to harden the epoxy. A drop of electrolyte was placed on top of the hole made in the counter electrode. If sealing was good, the drop would not go into the device because of the air pressure produced by the trapped air in between the counter electrode and photoanode. The whole device was kept in a small vacuum box (Figure 2.8B) and air was pumped out for 15s. When air was pumped 
in again, the electrolyte filled the vacuum space and the porous part of the dye-coated photoanode. The drilled hole was then filled with liquid Surlyn sealant again, and on the top of it, a drop of melted Crystalbond adhesive wax (Ted Pella, USA) was applied to seal the hole. Epoxy was placed on top of the cooled wax. Steps 7 and 8 are summarized in Scheme 2.1. Two approaches were adapted to measure I-V curves of samples: sandwich set-up and beaker set-up. Both schemes are shown in Figure 2.9. The beaker set-up is inexpensive, less time consuming, and can measure a large number of samples in a day that is necessary for statistical analysis of physical and chemical properties associated with a device. However, this set-up cannot provide the optimal efficiency because of the large distance between a PA and a CE to regenerate dye. Therefore, the sandwich cell set-up was used for optimization

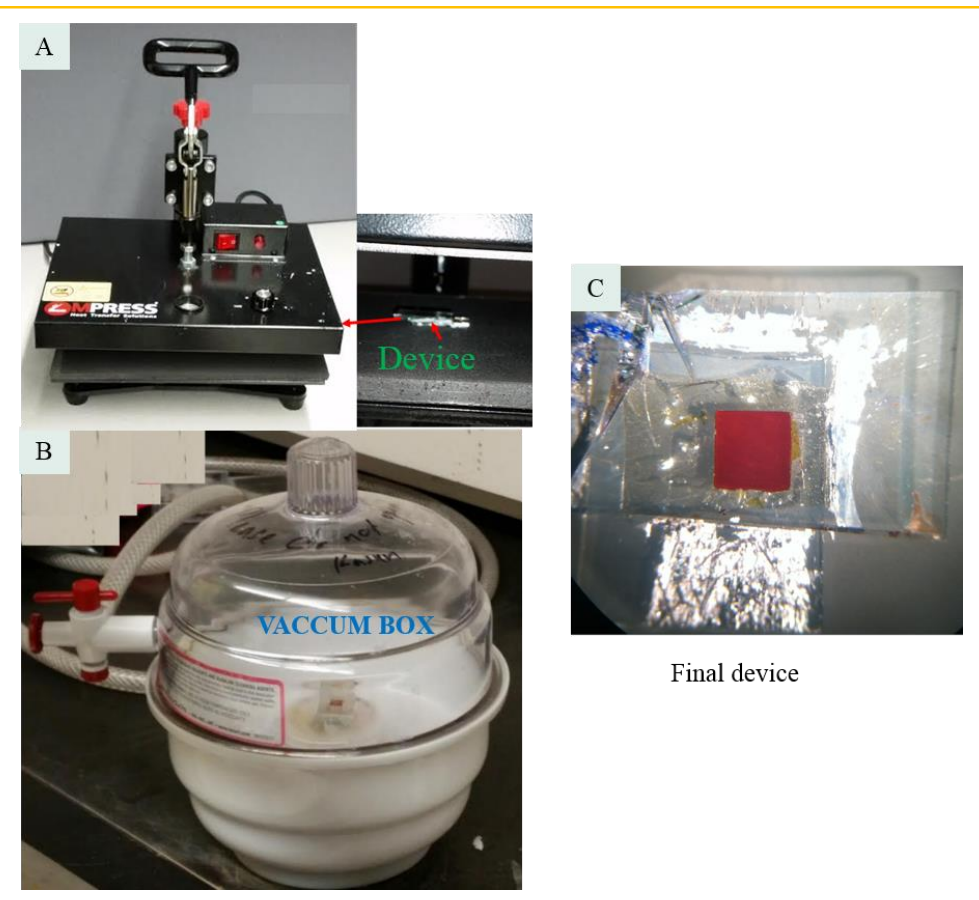

Figure 2.8. Sealing by thermal press (A), electrolyte filling by vacuum (B), and real final device (C).

of device performance. A similar approach was adopted to make PAs (Figure 2.9B) for the 
beaker set-up. The size of PAs in beaker set-up was $1 \mathrm{~cm} \times 5 \mathrm{~cm}$. since this size can well fit into a $1 \mathrm{~cm}$ x $5 \mathrm{~cm}$ glass cuvette.

\subsection{Chemical Modification}
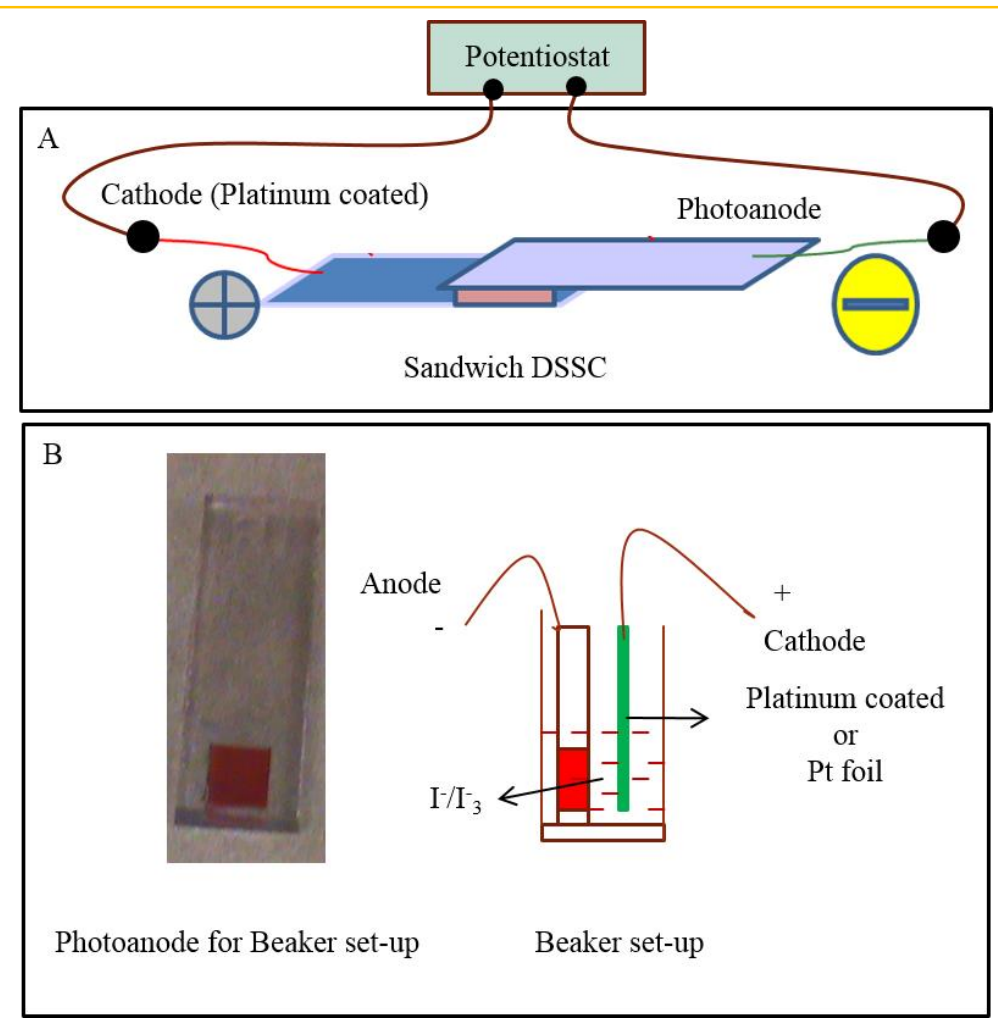

Figure 2.9. Sandwich cell (A) and Beaker cell (B).

Surface chemistry is a branch of chemistry that deals with the processes occurring at the interface between different phases of matter. Surface chemistry is applicable everywhere. Sir James Dewar ${ }^{34}$ found that charcoal can absorb very large quantities of gases such as oxygen and nitrogen, which is possible because bulk charcoal has fine reactive divisions to absorb. The necessity of soap/serf to lower surface tension and permeate into every corner of cloth and remove dirt from clothes is an obvious phenomenon of surface chemistry. The phenomena occurring at the interface may be physical (such as lowering 
surface tension or absorption of gases by charcoal), or chemical (such as removing pollutant from waste by titania surface, immobilization of nanoparticles or proteins on glass slides, etc.). ${ }^{35}$ When the chemical structure of macromolecules, such as proteins and nanoparticles, are modified by chemical attachment of other molecules or substances for different purposes, it is known as chemical modification. $\mathrm{TiO}_{2}$ anatase is an active catalyst and has been used for many purposes, such as water-splitting processes to produce storable energy sources (hydrogen), making artificial teeth, removing pollutants from the environment, and cleaning glass windows in a moisture environment. ${ }^{36}$

In this work, $\mathrm{TiO}_{2}$ is chemically modified to prepare stable and efficient dye-sensitized solar cells. The molecular linkers used for modifications were 3-aminopropyltriethoxysilane (APTES) and p-aminophenyltrimethoxysilane silane (APhS).

\subsection{Covalent Attachment of Dye onto $\mathrm{TiO}_{2}$}

Chemical bonds that involve sharing of electrons between atoms are called covalent bonds and are considered as a stable bond structure in chemistry. Carbon atoms have the excellent capacity to make covalent bond (s) with other atoms due to its half-filled valence orbital in addition to its capacity to form sp hybridized orbitals. Amide bonds are a family of covalent bonds formed from amines $\left(\mathrm{NH}_{2}\right)$ and $\mathrm{COOH} / \mathrm{COO}^{-}$groups. Amide bonds are strong enough to resist water due to hydrophobicity of the amine functional group. Direct formation of an amide bond is catalyzed by the addition of stoichiometric amounts of coupling reagents, such as dicyclohexylcarbodiimide, DCC in the presence of a base like $\mathrm{N}^{\prime} \mathrm{N}^{\prime}$-dimethylaminopyridine (DMAP) ${ }^{37}$ Glass $\left(\mathrm{SiO}_{2}\right)$ or n-type semiconductor oxides

contain native $\mathrm{OH}$ sites. If they lack $\mathrm{OH}$ sites, $\mathrm{OH}$ sites can be created by treating with 
Piranha $\left(\mathrm{H}_{2} \mathrm{O}_{2}: 3 \mathrm{H}_{2} \mathrm{SO}_{4}\right)$ solution. (Caution: Piranha is very corrosive and experiments should be done inside a hood). Free hydrogen from Piranha can react with oxygen to make $\mathrm{OH}$ sites. ${ }^{38}$ The $\mathrm{OH}$ sites can be functionalized with 3-aminopropyltriethoxysilane (APTES) to create a free standing amine $\left(\mathrm{NH}_{2}\right)$ layer on oxides, which can provide a platform for molecular engineering applicable to various purposes. Originally, APTES-functionalized glass slides were used to immobilize protein molecules for medical purposes. ${ }^{39}$ APTES can remain on the glass surface for a long period of time. When APTES-functionalized glass slides or semiconductor oxide mesoporous films are dipped into a solution containing stoichiometric amounts of DCC and DMAP and a carboxylic acid, amide bonds are formed. The amide bond can be observed in ATR-FTIR spectroscopy as a sharp peak around 1648 $\mathrm{cm}^{-1}$.

In this work, covalent modification of various electrodes with organic modifiers was performed in order to improve stability, dye-coverage, and efficiency of DSSCs.

\subsection{Linking Plasmonic Au Nanoparticles onto $\mathrm{TiO}_{2}$}

Interdisciplinary areas of research are crucial for the advancement of science. Interdisciplinary areas can create diverse areas of research directions. In metal nanoparticles, such as $\mathrm{Au}$ and $\mathrm{Ag}$, conducting electrons can be excited by photons to create surface plasmons. ${ }^{40}$ This is a good example of an interdisciplinary area of physics and chemistry. Collective oscillations of electrons in a plasmonic material in tune with electromagnetic waves can produce a resonance phenomenon called surface plasmon resonance, which offers tremendous applications in chemistry, (to produce hues of rose widow), medicine (to 
produce color change in biosensors such as home pregnancy tests), physics (to enhance power conversion efficiency in photovoltaics), and nanoscale lasers. ${ }^{35,40}$

In this work, surface chemistry was used to link $\mathrm{Au}$ NPs to semiconductor $\mathrm{TiO}_{2} \mathrm{NPs}_{\text {. }}$ This combination can alter the electrical, optical, thermal and chemical properties of both materials. Au NPs also offer very high reactive surface area for dye adsorption, fast electron transfer between molecules and nanoparticles, electron transport within mesoporous NPs in the thin film, and improved dye regeneration rate. ${ }^{41}$

\subsection{Characterization Techniques}

\subsubsection{Steady-state UV-vis Spectroscopy (UV-vis spectroscopy)}

UV-vis spectroscopy can measure electronic transitions in molecular species produced by absorbed photons from samples. In this work, UV-vis spectroscopy was used to study the amount of N719 dye adsorbed on different semiconductor and metal nanoparticles, such as $\mathrm{TiO}_{2}, \mathrm{Nd}_{2} \mathrm{O}_{3}$, and $\mathrm{Au}$ NPs. Cary win Bio-50 UV-vis spectrometer (Australia) was used to obtain absorption spectra. Diffuse reflectance/transmittance near IR spectroscopy (DR/TS near IR), was used to study the optical behavior of micronanocomposites. In DRS, samples may be thicker and light may get scattered /reflected multiple times through the sample before entering a detector. ${ }^{42}$ This light can carry a lot of information about the sample. In this study, DRS was used to calculate the band gap ( $\left.E_{g}\right)$ of a semiconductor nano-micro composite film. Since the incident light undergoes many processes inside samples, we cannot use the Beer-Lambert law. We used the Kubelka-Munk 
function to calculate Eg. A Perkin Elmer Lambda $950 \mathrm{UV}$-vis spectrometer was used for the measurements.

\subsubsection{Atomic Force Microscopy}

Soon after the discovery of atomic force microscopy (AFM) by Binnig in the early 1980s, AFM became very popular among scientists to study the physical properties of mater at the nanometer scale such as surface morphology, electrical, mechanical, magnetic, electrochemical, chemical binding interactions, and protein folding. ${ }^{43}$ The tip of an AFM can detect very small force down to the piconewton range in nanometer distances between the tip and the substrate where the matter to be analyzed is placed. This makes it possible for AFM to produce images at atomic resolution, which is not possible by SEM. In addition, AFM can equally be applied to conductive or non-conductive substrates unlike STM that is applicable to only conductive substrates.

In this research, AFM images were taken with the help of a Veeco Digital Instruments Nanoscope Illa Multimode scanning probe microscope (SPM) using a silicon tip from Nanoandmore, USA. The tip radius of curvature was $10 \mathrm{~nm}$ and all images were collected in non-contact mode.

\subsubsection{ATR-FTIR Spectroscopy}

Attenuated total reflectance-Fourier transform infrared spectroscopy [ATR-FTIR] works on the principle of total internal reflection of light. When the incident angle is greater than the critical angle for the interface of a sample and the transparent crystal on which the sample is mounted, laser light passed through the sample can make a series of multiple total internal reflections, which finally emerge from the sample. ${ }^{44}$ At each point of the total 
internal reflection sites on the interface, an evanescence wave is produced. This evanescence wave carries a lot of information about the sample, and is detected. ATR-FTIR is a powerful technique to obtain various interaction between species, similar to FTIR, however, it can reduce the time of sample preparation and data collection in comparison to transmission FTIR.

ATR-FTIR spectroscopy was used to detect various interaction modes between $\mathrm{TiO}_{2}$ and N719 dye, and to determine the quantity of N719 dye, focusing on the interaction between NCS ligand of $\mathrm{N} 719$ dye and $\mathrm{TiO}_{2}$. In addition, this technique is useful to find the interaction between $\mathrm{TiO}_{2}$ and APTES, $\mathrm{TiO}_{2} / \mathrm{APTES}$ and $\mathrm{Au}$ nano-particles, and $\mathrm{TiO}_{2}-\mathrm{Nd}_{2} \mathrm{O}_{3}$ nan-micro composition. An ATR-FTIR from Perkin Elmer Spectrum series 100, USA, was used to take the ATR-FTIR spectra of the samples.

\subsubsection{Scanning Electron Microscopy}

High energy electrons are being used to produce an image at the nanoscale, because of the wave nature of electrons. When high energetic electrons knock off inner core atomic orbitals electrons from the outer core go into vacant orbits releasing electromagnetic waves, usually in the form of characteristic x-rays. Production of x-rays depends on the composition of the matter, therefore, these electrons can be used to study the composition of matter. This technique is called energy dispersive analysis of $\mathrm{x}$-ray (EDAX). Sometimes these $\mathrm{x}$-rays, instead coming out of sample, knock off outer electrons. These electrons produced by $\mathrm{x}$-ray are Auger electrons, and the nature of the ejected electrons depends on the composition of matter. ${ }^{45}$ Therefore the dual nature of energetic electrons (particle and wave) can be use make images at nanoscale and study the composition of matter. In this work, SEM was used 
to study the morphological structure of nan-micro composite of $\mathrm{TiO}_{2}-\mathrm{Nd}_{2} \mathrm{O}_{3}$, and nanocomposite of smaller $\mathrm{Au}$ nanoparticles attached to bigger $\mathrm{TiO}_{2}$ nanoparticles through APTES. A Zeiss Supra 35 equipped with in-lens ion annular detector operating at an accelerating voltage $0.5-20,000 \mathrm{~V}$ was used to obtain the images.

\subsubsection{Electrochemical Impedance Spectroscopy}

In any photoelectrochemical cell (PEC), light can trigger a reaction and maintain a direct current in the device because of charge separation and transportation. When a small perturbation is produced in the electrochemical system, the excited electrons will see many options to move which depend on the PEC environment. ${ }^{46}$ When an alternate current (AC) with a certain frequency is passed through a system with a very small amplitude on the device biased with light at its $\mathrm{V}_{\mathrm{oc}}$, the motion of charge in different parts of the cell is determined by $\mathrm{AC}$ resistance (impedance), which depends on the frequency. This carries a lot of information about the system. ${ }^{47}$ The technique is called electrochemical impedance spectroscopy (EIS). A general sketch of EIS and the equivalent circuit used in this study is shown in Figure 2.10. The equivalent circuit is based on a transmission line of propagation, which states that there should be some entity (say voltage or current or frequency) which can smoothly flow through all components of a circuit. Figure 2.10A illustrates a DSSC.

Figure 2.10B describes the electrical circuit elements showing resistances (R) and capacitances $(\mathrm{C})$ that relevant in a DSSC according to the different parts of a cell. Figure 2.10C illustrates a Nyquist diagram, where an imaginary impedance is plotted against the real impedance developed in the device while an AC current with varied frequency flows through the device. 
In this work, EIS was deployed to explore charge transport in the interfacial region of $\mathrm{TiO}_{2}, \mathrm{TiO}_{2}-\mathrm{Au}$, and $\mathrm{TiO}_{2}-\mathrm{Nd}_{2} \mathrm{O}_{3}$ photoanodes. Electrochemical Impedance Spectroscopic

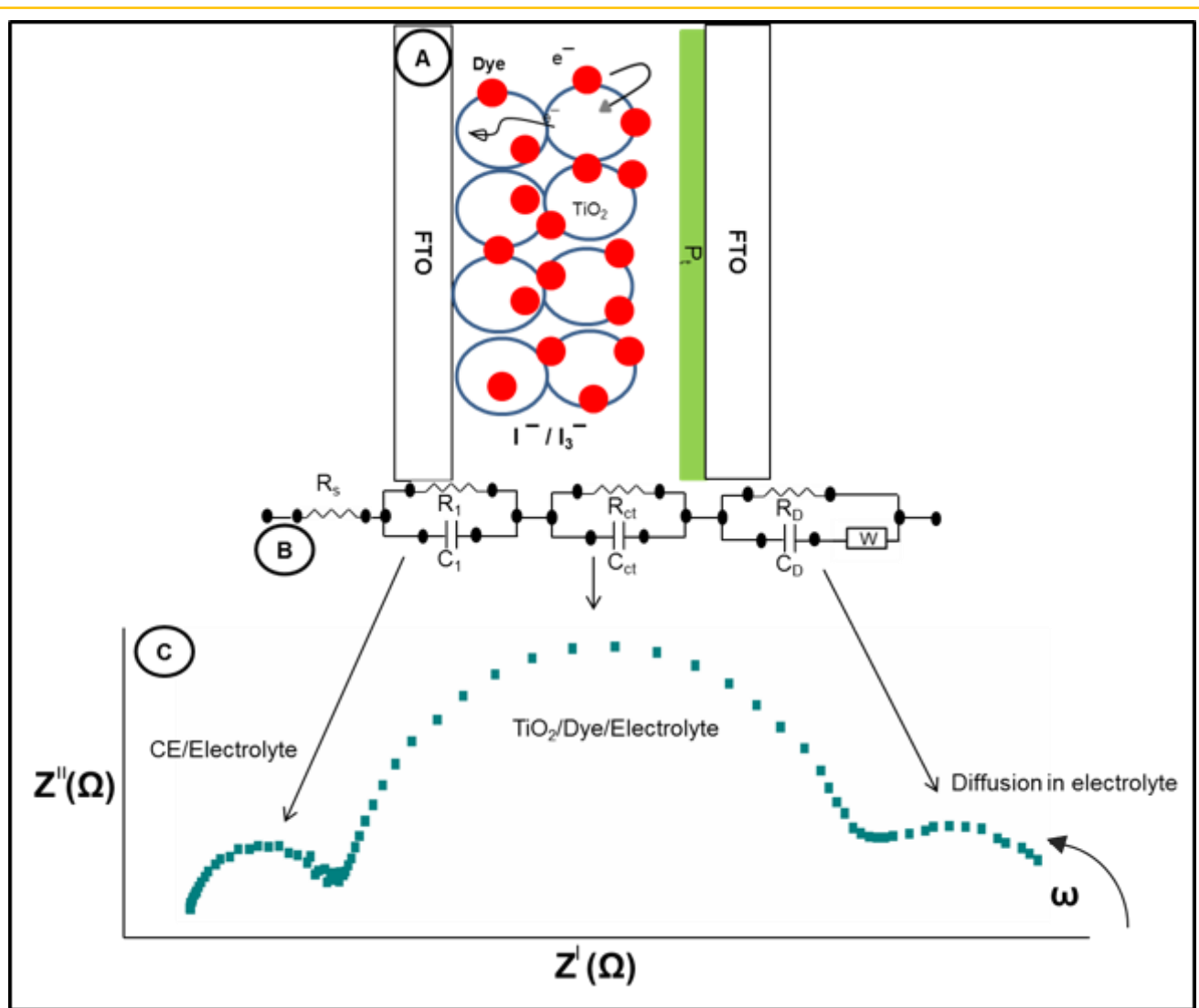

Figure 2.10. Equivalent circuit for EIS used in dye-sensitized solar cells. Real device (A), equivalent circuit (B), and Nyquist diagram for the device (C).

measurements were performed using a CHI 660a electrochemical workstation. A $10 \mathrm{mV}$ $\mathrm{AC}$ perturbation at an open circuit voltage was applied with a frequency range of $100 \mathrm{kHz}$ to $10 \mathrm{MHz}$. The photoanodes were maintained at an open circuit voltage by illuminating with white light produced from an optical fiber (Fiber Optic Illuminator Model 190, DolanJenner, USA). The DSSCs were connected in a two-terminal configuration. The photoanode was connected as the working electrode while the platinum counter electrode was connected to the reference and counter electrode leads. 


\subsubsection{Surface Profilometer}

A surface profilometer was used to measure the thickness of mesoporous films. The operating principle of a surface profilometer is similar to an atomic force microscope. A

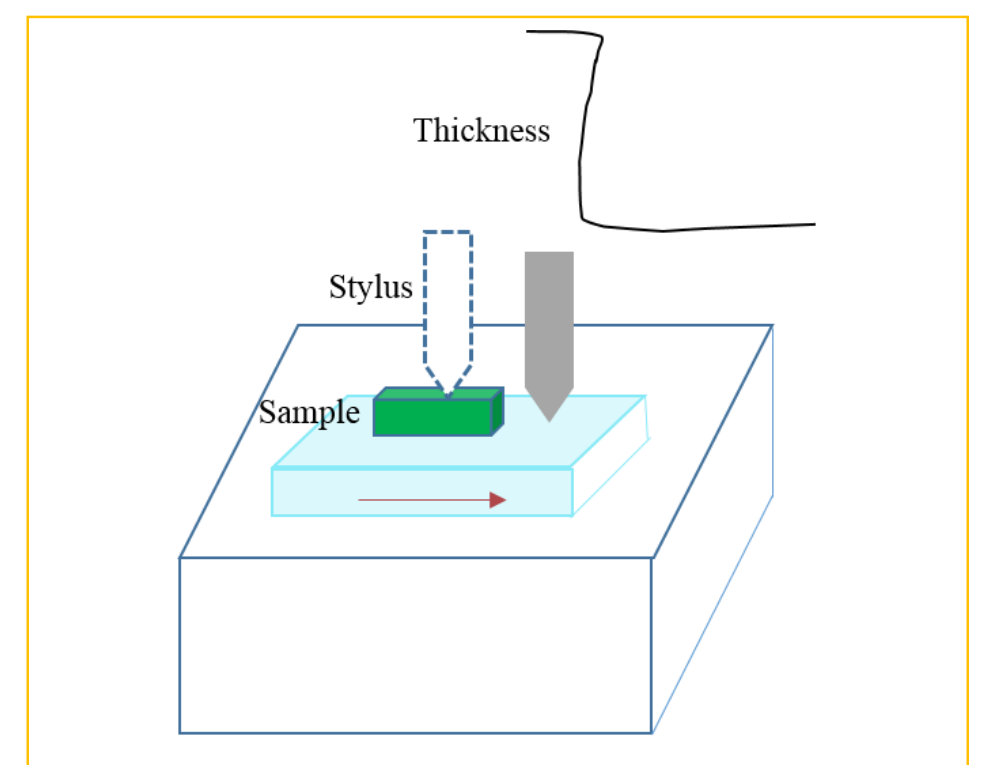

Figure 2.11. General sketch of a surface profilometer.

stylus is scanned across the edge of a sample horizontally from the sample surface to the substrate as shown in Figure 2.11. An electronic transducer attached to the stylus can convert each change in vertical distances on the sample into an electrical signal, and the electronic system on the machine can convert the analog signal to a digital signal to measure the thickness of the sample. ${ }^{48}$ Figure 2.11 shows a profilometer measuring the thickness of a sample.

\subsection{Photoelectrochemical Parameters}

\subsubsection{Photocurrent- Photovoltage Measurement}

Photoelectrochemical (PEC) parameters are the electrical behaviors developed in a solar cell device under illumination. By measuring a photocurrent-photovoltage (I-V) curve, 
all PEC parameters such as short circuit photocurrent density $\left(\mathrm{J}_{\mathrm{sc}}\right)$, open circuit photovoltage $\left(\mathrm{V}_{\mathrm{oc}}\right)$, power conversion efficiency $(\% \eta)$, and fill factor $(\mathrm{FF})$, can be obtained. Accurate measurement systems are crucial for reporting the PEC parameters. The global procedure for measuring the I-V curve comprises of illuminating the device with a simulated light source with less than $2 \%$ spectral mismatch factor and intensity equal to one sun and exposing the light only in a specific designated area of the photoanode (PA) by covering all other parts of the PA with light absorbing material (such as black tape). This avoids light piping effects when the photoanode is made on a glass substrate. ${ }^{49}$ Factors that can affect the I-V measurement are voltage sweep rate, direction of scans, electrical contacts made on the photoanode and counter electrode to draw current from the device, light source, junction temperature, instrumentation, and intensity. Specific care was taken to minimize any errors, and are discussed in each section below. I-V curves were generated by sweeping the potential negative at a constant sweep rate and measuring the current vs applied potential. A typical I-V curve is shown in Figure 2.12B.

\subsubsection{Solar Simulator Light Source}

The light source used in this work was produced from a Xenon-lamp from Newport, (Oriel, USA). The lamp was placed in a universal arc lamp housing which can work up to $500 \mathrm{~W}$ of power. The power supply to the lamp was separated from the rest of the electrical system to provide an uninterrupted power supply and to remove electrical noise that can arise from the contacts from the lamp panel to the power supply. The lamp cooling system 
was controlled by another power supply containing temperature sensors to control the mechanisms of the cooling fan, which can run even after the power supply to the lamp is turned off. The information about uniformity and mismatch factor of the light source is not
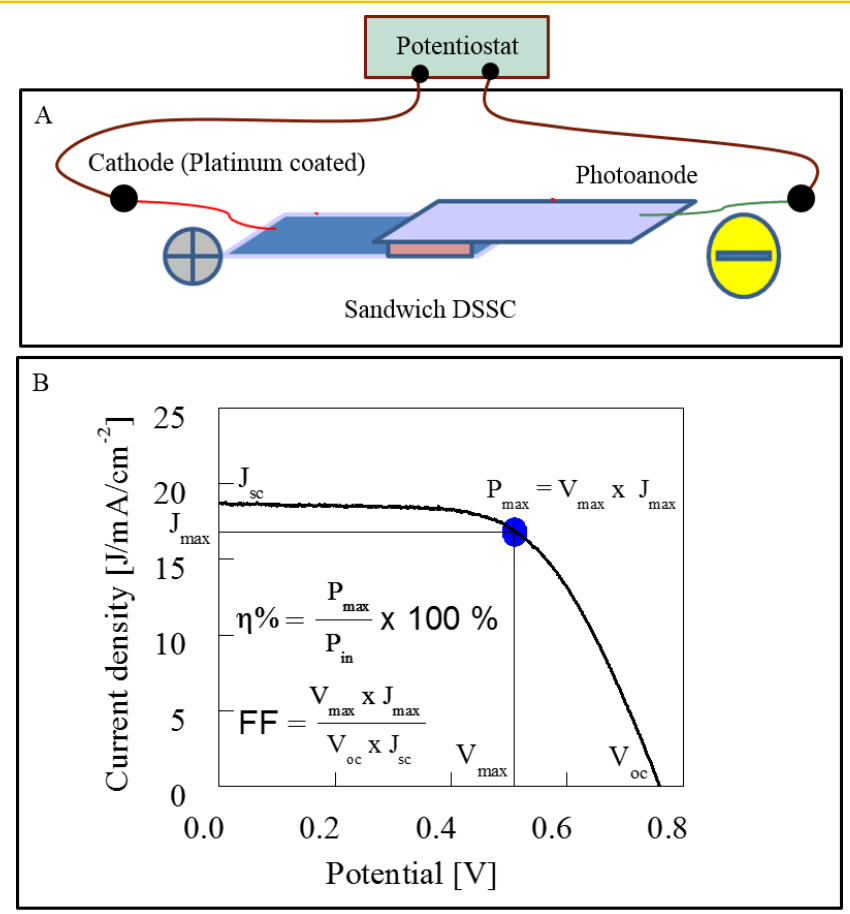

Figure 2.12. Calculation of PEC parameters. Device connected to a potentiostat (A) and I-V curve of the device after illumination with light (B).

provided. The light source was highly stable within $1 \%$ ripple factor. Light produced from the Xe lamp (Oriel, 300W) was filtered through AM 1.5 to produce a solar simulated light source. A calibrated silicon solar cell with uncertainty of $8 \%$ from Abet Technologies, Inc., (USA) with KG5 filter was used as a reference solar cell. The light source power was set to 1 Sun by making sure the reference cell measured $100 \mathrm{mV}$ according to a voltmeter when it was exposed to the simulated light. I-V curves of the devices were recorded using a Model 273A EG \& G Princeton Applied Research Potentiostat. An external bias voltage was scanned negative at a $20 \mathrm{mV} / \mathrm{s}$ scan rate. The voltage window used was from 0 to $-1 \mathrm{~V}$ and in some cases from +0.2 to $-1 \mathrm{~V}$. A homemade optical sample holder was used to mount the 
reference and solar cell devices. The solar cell devices were positioned on the center position where the reference cell was placed to calibrate the intensity. Figure 2.13 shows picture of the mounting of the solar cell devices and the reference silicon solar cell. The devices were masked by black tape, making the window area $0.16 \mathrm{~cm}^{2}$. The tape was used to seal the edges of the solar cell samples to prevent light piping effect. Figure $\mathbf{2 . 1 4}$ shows the arrangement of a device and solar simulated light source for appropriate measurement.

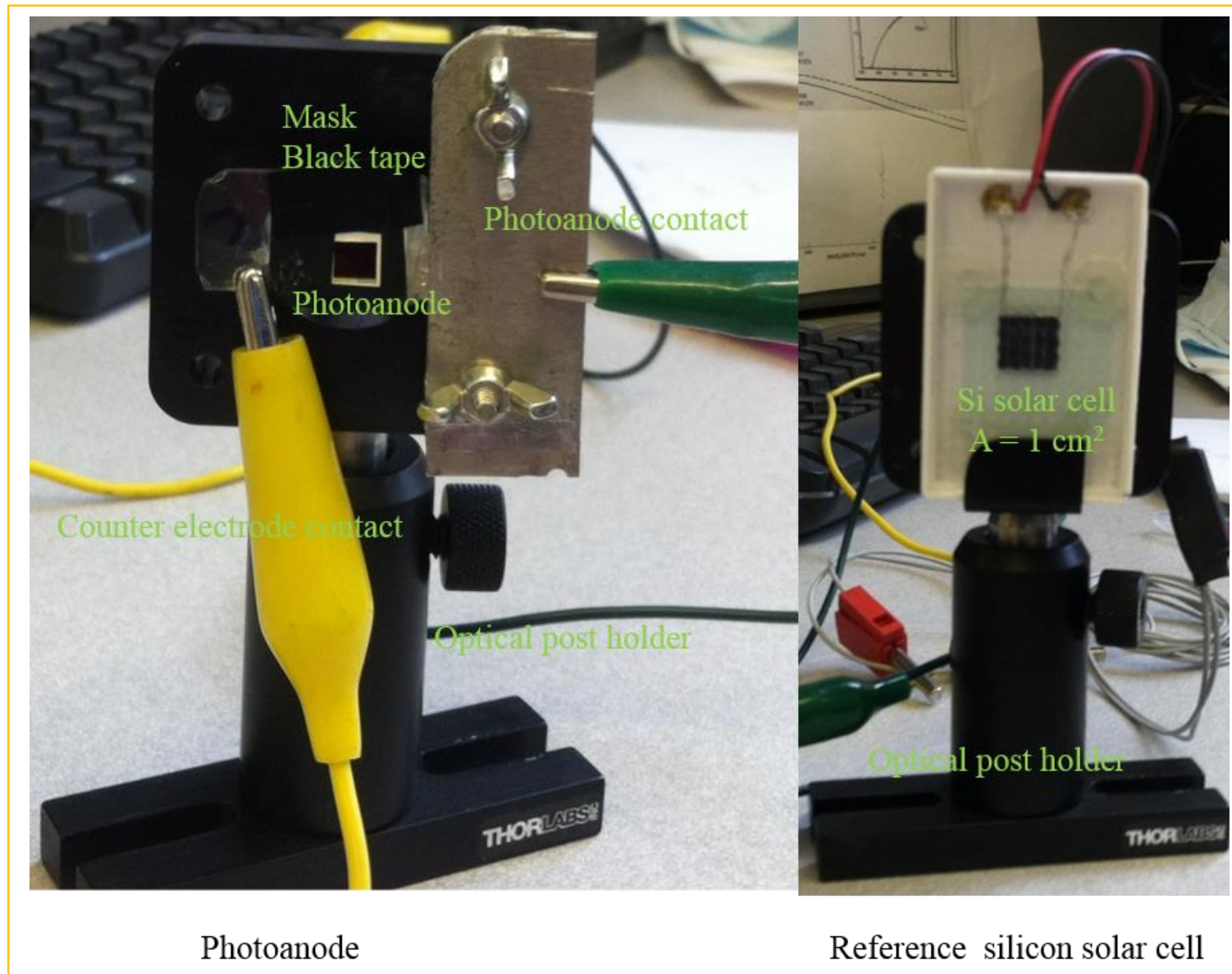

Figure 2.13. Homemade arrangement of sample and silicon reference for I-V measurements. 


\subsection{Ultrafast Transient Absorption Spectroscopy (UTAS)}

UTAS is a spectroscopic technique that employs ultrashort laser pulses to excite electrons to various excited states and then later the motion of these electrons are observed

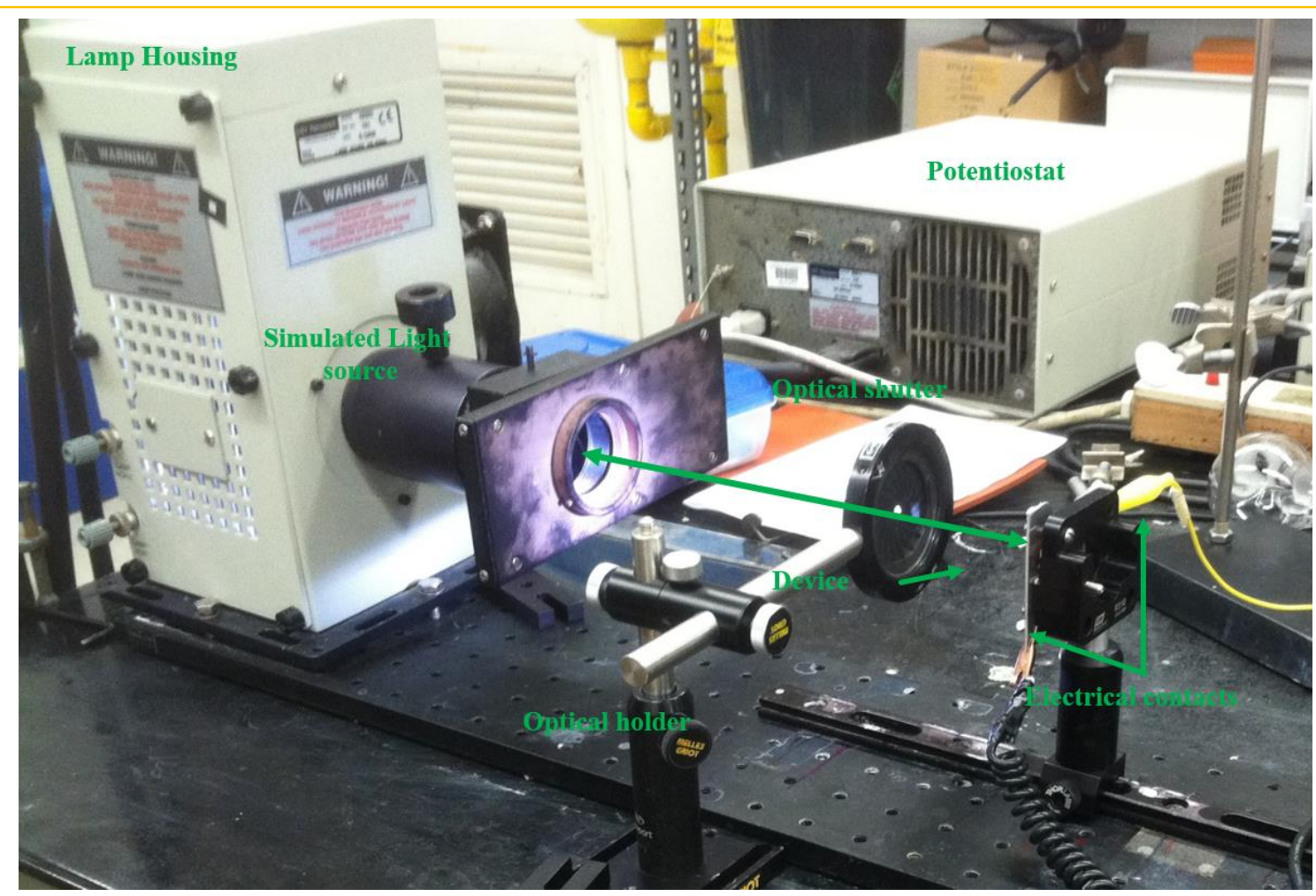

Figure 2.14. Measurement of I-V curves using solar simulated light

by an ultrashort laser pulse again. It is very a powerful technique to study the charge dynamics in various systems.

\subsubsection{Instrumentation}

Since the introduction of femtochemistry by Zewail in 1990, a lot of research related to the understanding of chemical reaction dynamics in real time frame (femto second) has been conducted. ${ }^{50}$ Injection of excited electrons from dye molecules to $\mathrm{TiO}_{2}$ semiconductor is in the femtosecond timescale. The injection kinetics is, therefore, crucial to understand the fundamental principle of DSSC technology, because power conversion efficiency 
depends on the temporal competiveness of injection, regeneration of dye, recombination of charge, charge transport in an electrolyte as well as in the mesoporous semiconductor film. The technique of ultrafast transient absorption spectroscopy (UTAS) is used in this case to study the charge injection dynamics of various devices fabricated in the laboratory.

\subsubsection{Theory of Pump-probe Measurements}

When a fraction of molecules of a substance is triggered to the excited state by a suitable wavelength of a laser light, it is known as a pump. The fate of excited electrons depend on the surroundings; they see various options before making any transition to other equilibrium states. They can go to the ground state by emission of electromagnetic waves (fluorescence), they can jump onto other materials in the interface (non-radiative, injection), or they can recombine with positive ions if the material is in contact with a liquid electrolyte. The average time during which a molecule can remain in an excited state is called fluorescence lifetime. The fluorescence lifetime is $\sim 1 \mathrm{~ns}$ in the case of N719 dye. If another laser light, usually with a very weak intensity, is sent through the molecules to examine the behavior of excited electrons, it is known as a probe. The probe pulse is deliberately set to be delayed from the pump laser pulse. The delay is made to be within the fluorescence lifetime of the excited electrons. In this way, the probe laser pulse can monitor the time evolution of excited state electrons and carry information about charge dynamics occurring at the interface.

A probe pulse can make different interactions with excited and ground state molecules. The difference between absorption of probe laser pulse by excited molecules and the absorption by ground state molecules is denoted by $\Delta \mathrm{A}(\lambda, \mathrm{t})$, where $\Delta \mathrm{A}(\lambda, \mathrm{t})$ is a function of wavelength and time. ${ }^{51}$ In general, $\Delta \mathrm{A}(\lambda, \mathrm{t})$ can carry four different processes: 
1) The first process is ground-state bleaching where the probe laser measures absorption without noticing the excited electrons. In this case, $\Delta \mathrm{A}(\lambda, \mathrm{t})$ is negative. No significant results can be obtained about the charge separation and charge dynamics.

2) In some cases, because of orbital structures, probe lasers may tune with stimulated emission of excited electrons, and there is always a population inversion in the system. The population inversion is not affected by the probe laser because the intensity of the probe laser is very weak. The ground state molecules are always less in number, therefore the value of $\Delta \mathrm{A}(\lambda, \mathrm{t})$ is also negative.

3) If the probe laser is able to absorb excited electrons to higher states, the absorption spectrum is greater in excited molecules than the absorption spectrum of ground-state. In this case, $\Delta \mathrm{A}(\lambda, \mathrm{t})$ is positive.

4) In some phenomena, excited states are long lived due to transition into a triple state, long-lived charge state, or isomerization, where their absorption spectrum is greater than the absorption spectrum when they are at the ground state. $\Delta \mathrm{A}(\lambda$, t) is also positive in this case. In $\mathrm{TiO}_{2} / \mathrm{N} 719$ system, there exists long-lived charge states in excited $\mathrm{N} 719$ dye and the conduction band of $\mathrm{TiO}_{2}$ is very near to the excited state. There is a possibility of injecting excited electrons to the conduction band. If the excited molecules react with other substances and make products, or excited molecules undergo various changes in orientations, the probe laser will be able to monitor the product and orientation of the molecules. 
This makes UTAS an attractive tool to understand charge transfer dynamics and chemical reactions kinetics.

In this study, process (3) is studied elaborately for various photoanodes made in the lab using $\mathrm{TiO}_{2}$, APTES, APhS, Au NPs, and N719 dye.

\subsubsection{Pump-probe Set-up}

All of the UTAS measurements were performed by using a Clark, MXR. Inc, USA. The experiment set up is shown in Figure 2.15. The TAPPS system uses a Clark-MXR CPA Ti:sapphire ultrafast laser (wavelength $=775 \mathrm{~nm}$, pulse duration $150 \mathrm{fs}$, pulse energy $1 \mathrm{~mJ}$ at $1 \mathrm{kHz}$ repetition rate). Output from the CPA is split and used to pump two noncollinear optical parametric amplifiers (NOPAs), which in total are able to cover a wavelength region from 450-1600 $\mathrm{nm}$. Part of the CPA output is also separated to pump a $3 \mathrm{~mm}$ thick sapphire plate to generate supercontinuum white-light (420-1600 nm). Output from the first NOPA at $530 \mathrm{~nm}$ wavelength was pulse-compressed to $30 \pm 5 \mathrm{fs}$ determined by an autocorrelation measurement. It was used as the pump light source. The pulse energy of the pump light is attenuated to below $0.2 \mu \mathrm{J}$ to avoid unwanted multiphoton excitation and thermal degradation of the samples. Both the white-light and the $860 \mathrm{~nm}$ output from the second NOPA were used as the probe light. In all measurements reported here, polarization of the pump and probe beams are parallel. The probe beam is focused onto the sample while the Pump beam is slightly defocused to ensure coverage of the probe beam. After the sample, the pump beam is blocked by a notch filter. When the $860 \mathrm{~nm}$ probe beam was used, its transmission sinnal was detected with a Si-photoanode (Thorlab DET10A) and amplified by 
a lock-in amplifier (SRS SR810), whereas with the white-light probe beam, the transmission is dispersed by a curved grating and detected by a linear array CMOS detector in the wavelength range $430-730 \mathrm{~nm}$. The time delay between pump and probe pulses $(\Delta \mathrm{t})$ is variable between 0 and $1.5 \mathrm{~ns}$ by moving a retroreflector on a computer-controlled translation stage that reflects the pump beam. An optical chopper revolving at $500 \mathrm{~Hz}$, half

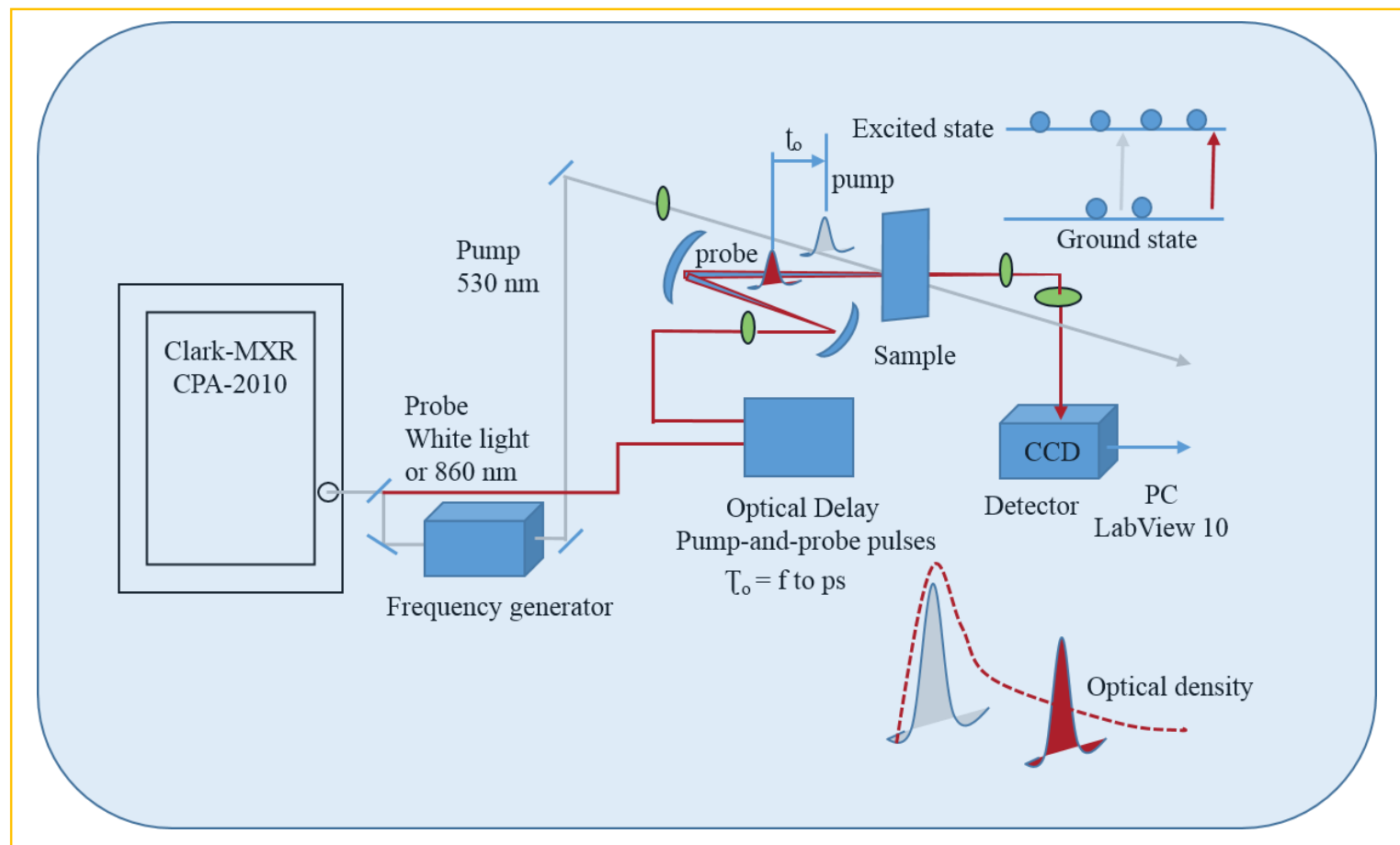

Figure 2.15. Pump-probe experimental procedure for the measurement of charge injection dynamics.

the repetition rate of the femtosecond laser, is used to modulate the pump beam and the TAPP signal is recorded as the change in optical density $(\Delta \mathrm{OD})$ with pump beam blocked

$$
\Delta \mathrm{OD}(\Delta t)=-\log \left[\frac{I^{*}(\Delta t)}{I_{0}}\right]
$$

and unblocked: where $\mathrm{I}_{0}$ and $\mathrm{I}^{*}$ are the probe beam transmission with the preceding pump beam blocked and unblocked, respectively. Time zero was determined by the transient absorption signal of R6G dye solution, and the group velocity dispersion was corrected. The TAPPS system is controlled by a LabVIEW (National Instruments) program. 


\subsection{4 $860 \mathrm{~nm}$ Probe for Decay Measurements}

To study the charge transfer dynamics in the $\mathrm{TiO}_{2} / \mathrm{N} 719$ system, processes (1) and (2) mentioned in section 2.7.2 should be avoided. Furthermore, the probe laser pulse should not excite any ground state molecules during the probing. The UV-vis absorption spectrum for the $\mathrm{FTO} / \mathrm{TiO}_{2} / \mathrm{N} 719$ shows that the absorption starts at $750 \mathrm{~nm}$ and lower wavelengths. Therefore, the choice of $860 \mathrm{~nm}$ laser pulse as a probe pulse is appropriate to observe charge transfer dynamics from $\mathrm{N} 719$ dye to $\mathrm{TiO}_{2}$. In addition, $\mathrm{TiO}_{2}-\mathrm{Au}$ photoanodes do not have optical activities beyond $700 \mathrm{~nm}$.

In this work, $860 \mathrm{~nm}$ probe laser was used to monitor the fate of excited electrons from $\mathrm{N} 719$ onto $\mathrm{TiO}_{2}$ on $\mathrm{FTO} / \mathrm{TiO}_{2} / \mathrm{N} 719$ photoanodes when the latter is illuminated with a pump laser at $530 \mathrm{~nm}$. The decay curve will solely due to charge transfer from $\mathrm{N}^{2} 19$ to $\mathrm{TiO}_{2}$, since the probe is measuring electrons in the excited state of N719 dye. Decay in the signal from those electrons at times shorter that emission indicate electron injection into the $\mathrm{TiO}_{2}$ conduction band. 


\section{CHAPTER 3}

\section{COVALENT MODIFICATION OF PHOTOANODES FOR STABLE DYE-SENSITIZED SOLAR CELLS}

This is the first research project during my Ph.D. study. The research has been published in Langmuir. The motivation behind this research was that N719 dye, which contains $\left(\mathrm{COOH} / \mathrm{COO}^{-}\right)$anchoring ligand groups, has several binding modes when it makes monolayer adsorption on $\mathrm{TiO}_{2}$. Some of these modes are physisorption and some are chemisorption. Chemisorption modes are essential for the stable interactive structure between $\mathrm{TiO}_{2}$ and $\mathrm{N} 719$. In this context, we describe the surface modification of $\mathrm{TiO}_{2}$ with 3-aminopropyltriethoxysilane (APTES) followed by covalent attachment of Ru-based N719 dye molecules to $\mathrm{TiO}_{2}$ through an amide linkage for use as photoanodes (PAs) in dyesensitized solar cells (DSSCs). Attenuated total reflectance-Fourier transform infrared spectroscopy (ATR-FTIR) confirms the surface chemistry between the $\mathrm{TiO}_{2}$ and dye. The photovoltaic efficiency of DSSCs with covalently-linked dye is $2.8 \pm 0.4 \%$ as compared to $5.8 \pm 0.6 \%$ for traditionally prepared DSSCs, where dye is adsorbed to $\mathrm{TiO}_{2}$ by direct immersion. While lower, the efficiency of PAs with covalently silane-linked dye did not change after storage for more than 60 days in air, whereas the traditionally-prepared DSSCs

decreased to less than $2 \%$ in one week and to $0 \%$ after twenty days. FTIR and UV-vis characterization of the traditional and covalently-linked dye suggests that covalent linkage improves stability by preventing the loss of thiocyanato ligands on the dye, which 
presumably occurs by exchange with atmospheric water. PAs with covalently-linked dye are also more stable towards water, acid, heat, and UV light compared to traditionallyprepared PAs as shown by UV-vis and FTIR spectroscopy measurements before and after the various treatments. The covalently-attached dye was also more stable compared to other modified PAs with dye attached non-covalently through electrostatic or hydrogen bonding interactions. The dramatic enhancement in stability with covalently-bound dye could be beneficial for future practical DSSC applications.

\subsection{Introduction}

Due to the tremendous need for renewable sources to fulfill the ever-increasing energy demand from human society, dye-sensitized solar cells (DSSCs) $)^{20,21}$ have been widely explored as a potentially low-cost alternative to silicon solar cell technology.$^{30} \mathrm{An}$ increase in efficiency to $15 \%{ }^{11}$ and production of cells that are stable for 20 years ${ }^{31}$ would be a great step toward the commercialization of DSSCs.

There are many factors that affect the efficiency of DSSCs, especially the electron transport processes occurring at the semiconductor/dye, dye/electrolyte (redox couple), and semiconductor/electrolyte interfaces, because these are where electron injection, ${ }^{52}$ dye regeneration,$^{53}$ and the competing processes of electron recombination occur. ${ }^{54}$ All of these processes and the stability ${ }^{55}$ of the semiconductor/dye/electrolyte junction rely heavily on the nature of the dye, the dye functionality, the environment, and the dye/semiconductor interaction. ${ }^{56,57}$

It is generally desirable to prepare a porous, high surface area semiconductor film with monolayer coverage of dye that is attached through a strong anchoring group for high 
stability and strong coupling for fast electron injection..$^{58}$ The dye coverage is important because low coverage may reduce the amount of light collected (lower efficiency), whereas a coverage too high could cause aggregation or multilayers of dye that may reduce the efficiency due to poor coupling between the dye and semiconductor or poor regeneration kinetics. ${ }^{59-61}$ The optical properties and the binding characteristics of the dye have been altered by varying the level of protonation, ${ }^{62}$ adding donor substituents, ${ }^{63}$ and varying the number of binding groups ${ }^{64}$ which are important for stability and controlling the photoelectrochemical properties. The distance between the dye and semiconductor is another important variable controlled by the chemistry at the interface. ${ }^{65-67}$ It is generally thought that the excited electron should be localized on the anchoring group to maximize dye-semiconductor coupling, ${ }^{68}$ but it is also important to reduce kinetic redundancy. ${ }^{69}$ Usually a larger distance hinders the rate of electron injection, which is not desirable, but it can also decrease recombination, which is a benefit. ${ }^{54,56,69}$ Interestingly, Galoppini et al. observed electron injection on the nanosecond time scale over long distances of about 18 $\mathrm{nm}$ using a carboxylate anchor group and a spacer group, suggesting that direct coupling is not always necessary. ${ }^{65}$

There are several different types of metal complex based dyes and different methods for attaching them to semiconductor surfaces. ${ }^{58}$ The most common interaction reported is through the carboxylate/carboxylic acid group $\left(\mathrm{COOH} / \mathrm{COO}^{-}\right)$, where there are five possible modes of interaction as shown in Scheme 3.1 for N719 dye with $\mathrm{TiO}_{2}{ }^{70}$ The attachment is usually described as a combination of bidentate bridging and hydrogen-bonding modes, based on the location of the carboxylate bands in an infrared spectrum. ${ }^{62,64,70,71}$ Other potential anchoring groups include $\mathrm{B}(\mathrm{OH})_{2}, \mathrm{PO}(\mathrm{OH})_{2}, \mathrm{SO}_{2}(\mathrm{OH}), \mathrm{OH}, \mathrm{NO}_{2}$, and $\mathrm{SiCl}_{3}$ as 
recently described computationally for a functionalized $\mathrm{N} 3$ dye on anatase $\mathrm{TiO}_{2}(101) .^{72}$ Meyer and co-workers used an acetylacetonate group for linking $\mathrm{Ru}$ polypyridyl dye to Scheme 3.1. Different possible interactions between $\mathrm{TiO}_{2}$ and $\mathrm{N} 719$ dye molecule.

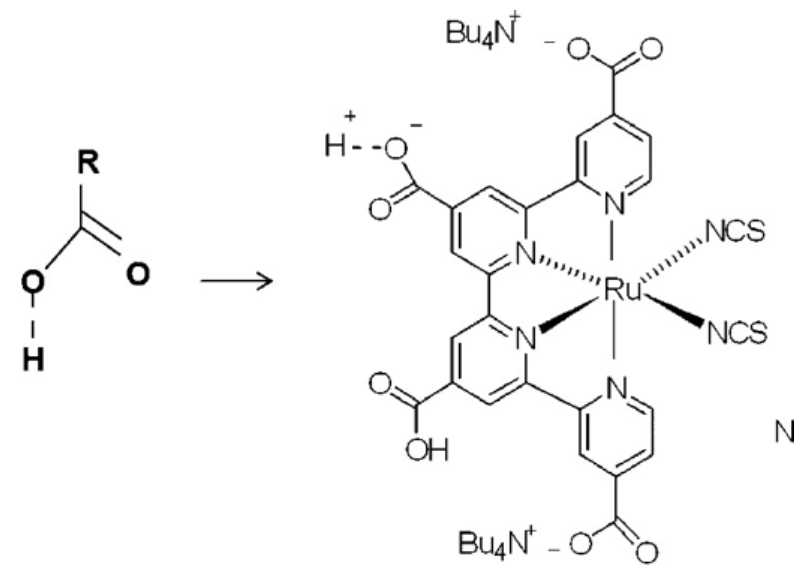

N719 Dye
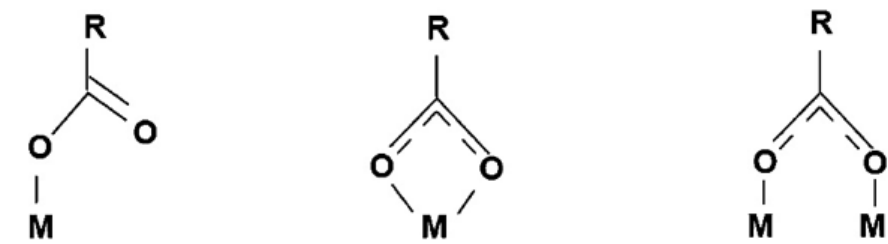

\section{Unidentate}

Bidentate chelating

Bidentate bridging
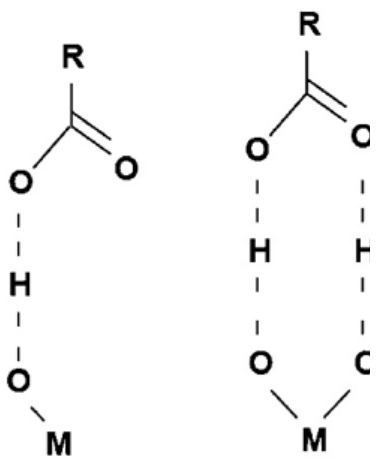

$\mathrm{TiO}_{2}$, but with lower coverage compared to $\mathrm{COOH}$ groups. ${ }^{73}$ The phosphonate group has also been widely used for the adsorption of various sensitizers because it binds more strongly and with greater stability compared to $\mathrm{COOH} / \mathrm{COO}^{-},{ }^{74,75}$ although it exhibits slower electron injection compared to N3 or N719 with carboxylate groups. ${ }^{74}$ Recently, both groups on the same dye were used to exploit the benefits of both, especially for use in aqueous solution. ${ }^{76}$

Covalent bonding of dye to various metal oxide surfaces is another attachment strategy that has been used on electrode surfaces since the 1970s and is different from the chemisorption that occurs with the common carboxylate and phosphonate anchor groups. The most common occurs by either (1) silanization of dye molecules and direct attachment to the metal oxide through a $\mathrm{M}-\mathrm{O}-\mathrm{Si}-\mathrm{D}$ bond, where $\mathrm{D}$ is the dye and $\mathrm{M}-\mathrm{O}$ is the metal 
oxide, ${ }^{77-80}$ or (2) silanization of the metal oxide followed by attachment of the dye through organic coupling, such as amide bond formation ${ }^{81-83}$ or a replacement reaction.$^{84}$ Recently, Brennan et al. used silatranes ${ }^{85}$ and Hamers and co-workers used photoelectrochemical grafting ${ }^{86}$ and click chemistry ${ }^{87}$ to attach sensitizers covalently to metal oxide semiconductors.

The $\mathrm{TiO}_{2} /$ dye interface is critically important for good long-term DSSC stability. ${ }^{31,55,88-90}$ Grätzel and co-workers demonstrated excellent stability for two sealed DSSC cells that were irradiated continuously for $7000 \mathrm{~h}$ at $100 \mathrm{~mW} / \mathrm{cm}^{2}$, even when raising the temperature to $75{ }^{\circ} \mathrm{C}$ for $700 \mathrm{~h} .{ }^{91}$ Although these results are promising, there are conditions when DSSC stability is a major issue, and several publications express serious concern about the long-term stability for 15-20 years. ${ }^{92,93}$ There are many potential stability issues, but degradation ${ }^{55,94,95}$ and desorption ${ }^{76,90,96}$ of dye are among the biggest concerns. It is well-known that Ru-based dye is not stable in air when adsorbed to $\mathrm{TiO}_{2}$, making it necessary to seal the cell immediately. ${ }^{91,92,94,97}$ Hagfeldt and co-workers showed that ligand exchange between atmospheric $\mathrm{H}_{2} \mathrm{O} / \mathrm{OH}^{-}$and NCS occurs on $\mathrm{TiO}_{2}$ adsorbed dye in air, which is accelerated by visible light, heat, and possibly UV light. ${ }^{94}$ This can occur in electrolytes as well, especially if water is present, leading to desorption of dye or optical changes. Poor air and water stability can be issues in the processing of DSSCs. ${ }^{94}$ Dye can undergo photo-oxidation, but the presence of $\mathrm{I}^{-}$in the electrolyte is thought to prevent that from occurring appreciably during operation. ${ }^{91}$ Under illumination, the N719 dye undergoes a chemical reaction with iodine to form $\mathrm{I}_{2} \mathrm{SCN}^{-55}$ and can decompose into fragments, including $\mathrm{Ru}(\mathrm{bpy})_{2}(\mathrm{CN})_{2} .{ }^{92}$ The $\mathrm{TiO}_{2}-\mathrm{COOH}$ bond can cleave in acidic solution ${ }^{55}$ Images 
of photocurrent and photovoltage showed obvious degradation of dye within $24 \mathrm{~h}$ after the preparation of DSSCs. ${ }^{95}$

It is clear that further improvements in DSSC efficiency and stability are still needed to realize commercial applications in the future. Here we describe DSSCs composed of PAs with N719 dye covalently attached by silanization of $\mathrm{TiO}_{2}$ with 3aminopropyltriethoxysilane (APTES) and then attachment of dye through a covalent amide bond between the $\mathrm{COOH} / \mathrm{COO}^{-}$groups of the dye and the $\mathrm{NH}_{2}$ group of $\mathrm{APTES}^{81-83,98}$ We compare the efficiency and stability of these PAs to those of the traditional $\mathrm{TiO}_{2} / \mathrm{N} 719$ PAs and to those with $\mathrm{TiO}_{2} / \mathrm{APTES} / \mathrm{N} 719$ PAs without covalent amide attachment. It is important to note the pre-modification ${ }^{99}$ and post-modification ${ }^{100}$ of PAs by APTES have been studied previously to improve the efficiency of DSSCs using Ru-based dye, but these did not involve subsequent covalent amide bond formation as shown in this work. This is the first study of silanization and amide bond formation with the N719 dye to our knowledge. We show that the covalent bonding strategy dramatically improves the stability under a variety of conditions important for DSSC operation and processing with very little decrease in the efficiency.

\subsection{Experimental Details}

Preparation of $\mathrm{TiO}_{2}$ film on FTO: Dyesol $\mathrm{TiO}_{2}$ paste was used as received without further treatment. For $\mathrm{P} 25 \mathrm{TiO}_{2}$ powder, a mixture of $280 \mathrm{mg}$ of ethyl cellulose, $1500 \mathrm{mg}$ of terpineol and $430 \mathrm{mg}$ of $\mathrm{TiO}_{2}$ nano powder were placed in a clean porcelain mortar and ground with a pestle for at least $1 \mathrm{~h}$ until it became a sticky fine paste. The paste (Dyesol or P25) was deposited with an area of $0.25 \mathrm{~cm}^{2}$ on FTO slides by the doctor blade 
method. This was used for all UV-vis, ATR-FTIR, and glass cell data in the paper. A 0.25 $\mathrm{cm}^{2}$ area was made on a $90 \mathrm{~T}$ screen printer and $\mathrm{TiO}_{2}$ was deposited on the FTO slide two times to obtain a thickness of about $7 \mu \mathrm{m}$ for photovoltaic measurements made on sandwich cells. The deposited paste was sintered at $500{ }^{\circ} \mathrm{C}$ for at least $1 \mathrm{~h}$ in a $1300 \mathrm{BL}$ Barnstead Thermolyne Furnace. This is referred to as an $\mathrm{FTO} / \mathrm{TiO}_{2}$ electrode.

Functionalization of $\mathrm{TiO}_{2}$ film: The $\mathrm{TiO}_{2}$ film on FTO $\left(\mathrm{FTO} / \mathrm{TiO}_{2}\right)$ was placed inside a solution containing $10 \mathrm{~mL}$ of IPA, $100 \mu \mathrm{L}$ of APTES, and 3-4 drops of nanopure water. The solution was placed in a water bath at $70{ }^{\circ} \mathrm{C}$ for $30 \min ^{101}$ The electrode was removed and rinsed thoroughly with IPA and dried under a $\mathrm{N}_{2}$ stream for $1 \mathrm{~min}$. This is referred to as an $\mathrm{FTO} / \mathrm{TiO}_{2} / \mathrm{APTES}$ electrode. Figure 3.1 and Figure 3.2 confirm that APTES was well attached on $\mathrm{TiO}_{2}$ by ATR-FTIR and XPS data, respectively. The

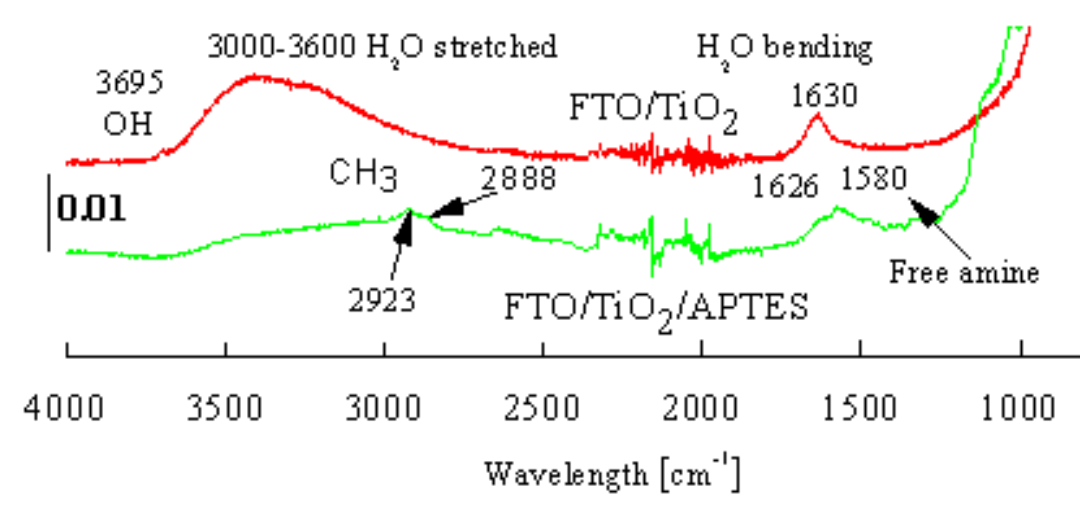

Figure 3.1 ATR-FTIR spectra for $\mathrm{FTO} / \mathrm{TiO}_{2}$ (red) and $\mathrm{FTO} / \mathrm{TiO}_{2} / \mathrm{APTES}$ (green).

disappearance of bending mode $\left(1630 \mathrm{~cm}^{-1}\right)$ and stretched mode $\left(3000-3600 \mathrm{~cm}^{-1}\right)$ along with $\mathrm{OH}$ site $\left(3695 \mathrm{~cm}^{-1}\right)$ of adsorbed water from $\mathrm{TiO}_{2}$ spectrum after functionalization with APTES confirms the chemical modification of $\mathrm{TiO}_{2}$. The $1580 \mathrm{~cm}^{-1}$ peak correlates to a free amine. Appearance of Si 2P peak and two nitrogen peaks in the XPS data shows the presence of APTES on $\mathrm{TiO}_{2}$. 
Dye sensitization of $\mathrm{TiO}_{2}$ film: Sensitization of the $\mathrm{FTO} / \mathrm{TiO}_{2}$ or $\mathrm{FTO} / \mathrm{TiO}_{2} / \mathrm{APTES}$ electrodes was achieved by immersing into a (1) $0.3 \mathrm{mM}$ solution of N719 dye in a 1:1 mixture of acetonitrile and $t$-butanol, (2) $0.3 \mathrm{mM}$ solution of N719 dye in dichloromethane, or (3) solution of $0.3 \mathrm{mM} \mathrm{N719}$ dye, $20 \mathrm{mM}$ DCC, and $20 \mathrm{mM}$ DMAP in

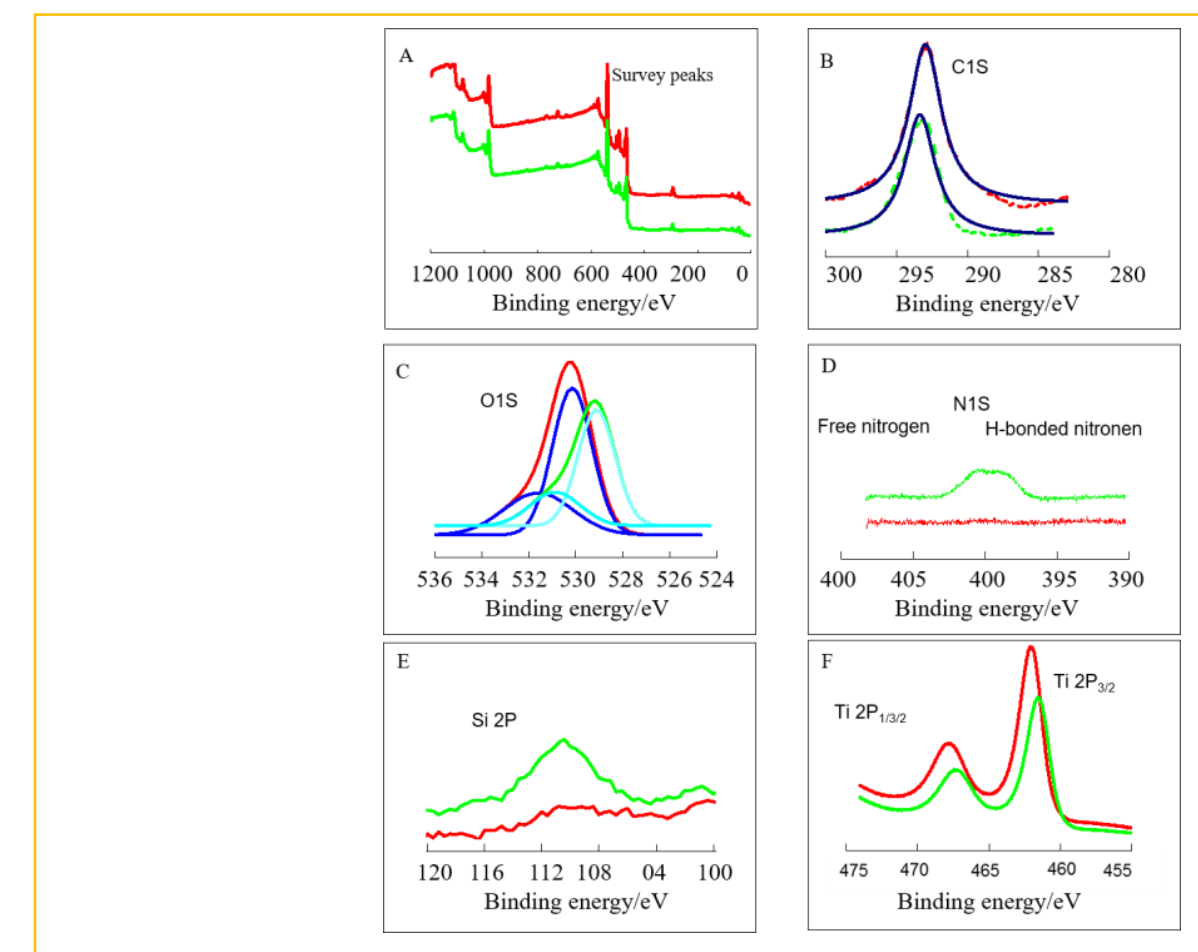

Figure 3.2. XPS spectra of $\mathrm{FTO} / \mathrm{TiO}_{2}$ (red) and FTO/TiO $/$ /APTES (green). Other colors are the fitted curves. (A) General survey, (B) carbon peak (C1S, 284.1 eV), (C) oxygen (O1S, 529$531 \mathrm{eV}$ ), (D) nitrogen (N1S, free $401 \mathrm{eV}, \mathrm{H}$-bonded $398 \mathrm{eV})$, (E) silicon (2P, $110 \mathrm{eV})$, and titanium ( $\mathrm{TiP}_{1 / 2}, \mathrm{TiP}_{3 / 2} 467$ and $462 \mathrm{eV}$, respectively).

dichloromethane. ${ }^{101}$ The electrodes were soaked in one of these three different dye solutions for $24 \mathrm{~h}$. Solution (3) should lead to covalent attachment of dye through an amide bond with the TO/TiO $/$ APTES electrodes with DCC as a catalyst and DMAP acting as a base to deprotonate the carboxylic acid groups of the N719 dye. We name the electrodes not functionalized with APTES as $\mathrm{FTO} / \mathrm{TiO}_{2} /$ Dye (NC-1), FTO/TiO $/$ /Dye $(\mathrm{NC}-2)$, and $\mathrm{FTO} / \mathrm{TiO}_{2} /$ Dye (NC-3), where $\mathrm{NC}$ stands for "non-covalent" and the number refers to the solution used for dye attachment. The APTES-functionalized electrodes are termed as 
FTO/TiO $/$ /APTES/Dye (NC-1) FTO/TiO $_{2} / \mathrm{APTES}_{\text {D }}$ ye (NC-2), and FTO/TiO $/$ /APTES/Dye (C-1), where NC is as before and C stands for "covalent". The number again refers to the solution used for dye attachment.

Photoactivity measurements: The N719 dye-sensitized photoanode (PA) acted as the working electrode. A counter electrode was produced by coating an FTO slide with a drop of Platisol (Solaronix) and heating to $500{ }^{\circ} \mathrm{C}$ for $20 \mathrm{~min}$. Both the working electrode and counter electrode were silver painted on the edges of the conductive sides to produce good electrical contact. Photoactivity was measured using two different set-ups; a sealed sandwich cell and open glass cell. Sealed sandwich cells were prepared by placing Surlyn (plastic polymer) between the dye-coated working electrode and $\mathrm{Pt}$ - coated counter electrode with a spacer and heating at $125{ }^{\circ} \mathrm{C}$ for ten minutes to seal them together. After injecting a drop of electrolyte in between the two cells, the cell was further sealed with wax. For the open glass cell, the working electrode and the counter electrode were kept in a $1 \mathrm{~cm}$ glass cuvette connecting each electrode with copper alligator clips. $3 \mathrm{~mL}$ of Iodolyte-50 was placed into the cuvette. Either cell was illuminated with simulated 1.5 AM solar light (Newport Oriel, USA) at an intensity of $100 \mathrm{~mW} / \mathrm{cm}^{2}$. Current-voltage curves were obtained by applying an external bias between the two electrodes of the cell and measuring the current with a Model 273A EG \& G Princeton Applied Research Potentiostat. The photon flux was measured by a power meter (Thorlab, USA). All photocurrent and photovoltage curves were measured using PAs with the P25 Degussa $\mathrm{TiO}_{2}$ paste.

Spectroscopic characterization: To characterize the surface attachment of N719 dye to the $\mathrm{TiO}_{2}$ films using the different attachment functionalization and different solvents, we measured the absorbance spectrum of PAs with a Varian Cary 50 Win UV-vis spectrophotometer (Australia) and a Perkin Elmer Spectrum series 100 attenuated total 
reflectance Fourier transform infrared (ATR-FTIR) spectrometer. The UV-vis measurements were performed by placing PAs prepared from Dyesol $\mathrm{TiO}_{2}$ paste in the light path of the instrument in air and referencing the absorbance to an $\mathrm{FTO} / \mathrm{TiO}_{2}$ slide with no dye. For ATR-FTIR measurements, PAs made from P25 degussa $\mathrm{TiO}_{2}$ or Dyesol $\mathrm{TiO}_{2}$ paste were placed upside down onto the ZnSe crystal in the ATR-FTIR spectrometer and held at a pressure of 80 psi. The samples were scanned from 400 to $4000 \mathrm{~cm}^{-1}$ with 20 scans averaged per sample with a resolution of $4 \mathrm{~cm}^{-1}$. We compared spectra of the PAs before and after they were 1) exposed to open air for 20 days, 2) placed in water, 3) placed in a solution of $28 \mathrm{mM}$ acetic acid, 4) heated to $140{ }^{\circ} \mathrm{C}$ in open air and in the dark, 5) heated to $140{ }^{\circ} \mathrm{C}$ along with exposure to a 4 watt 254/364 nm UVGL-15 Compact UV Lamp (Upland, CA, USA). These studies were performed to test the stability of the dye attached by the different methods.

\subsection{Results and Discussions}

Photoanode Preparation: For this project we prepared six different types of photoanodes (PAs) as shown in Scheme 3.2. Step A for all PAs was the deposition of $\mathrm{TiO}_{2}$ on the FTO (P25 degussa or Dyesol). The next step for the top three anodes was the adsorption of dye in the three different solutions shown, labeled as steps A1, A2, and A3. The PAs are named $\mathrm{FTO} / \mathrm{TiO}_{2} /$ Dye (NC-1), FTO/TiO 2 Dye (NC-2), and FTO/TiO $2 / \mathrm{Dye}$ (NC-3), respectively, where NC indicates "non-covalent" attachment and the number represents the solution $(1,2$, or 3$)$. The three electrodes on the bottom in Scheme 3.2 underwent a second step $\mathrm{B}$ involving functionalization of $\mathrm{TiO}_{2}$ with APTES before steps 
B1, B2, or B3, which exposed them to dye in the same three solutions. These PAs are named FTO/TiO $2 /$ APTES/Dye (NC-1, FTO/TiO $2 /$ APTES/Dye (NC-2), and FTO/TiO $2 /$ APTES/Dye $^{2}$

Scheme 3.2. Preparation of different types of photoanodes and their nomenclature.

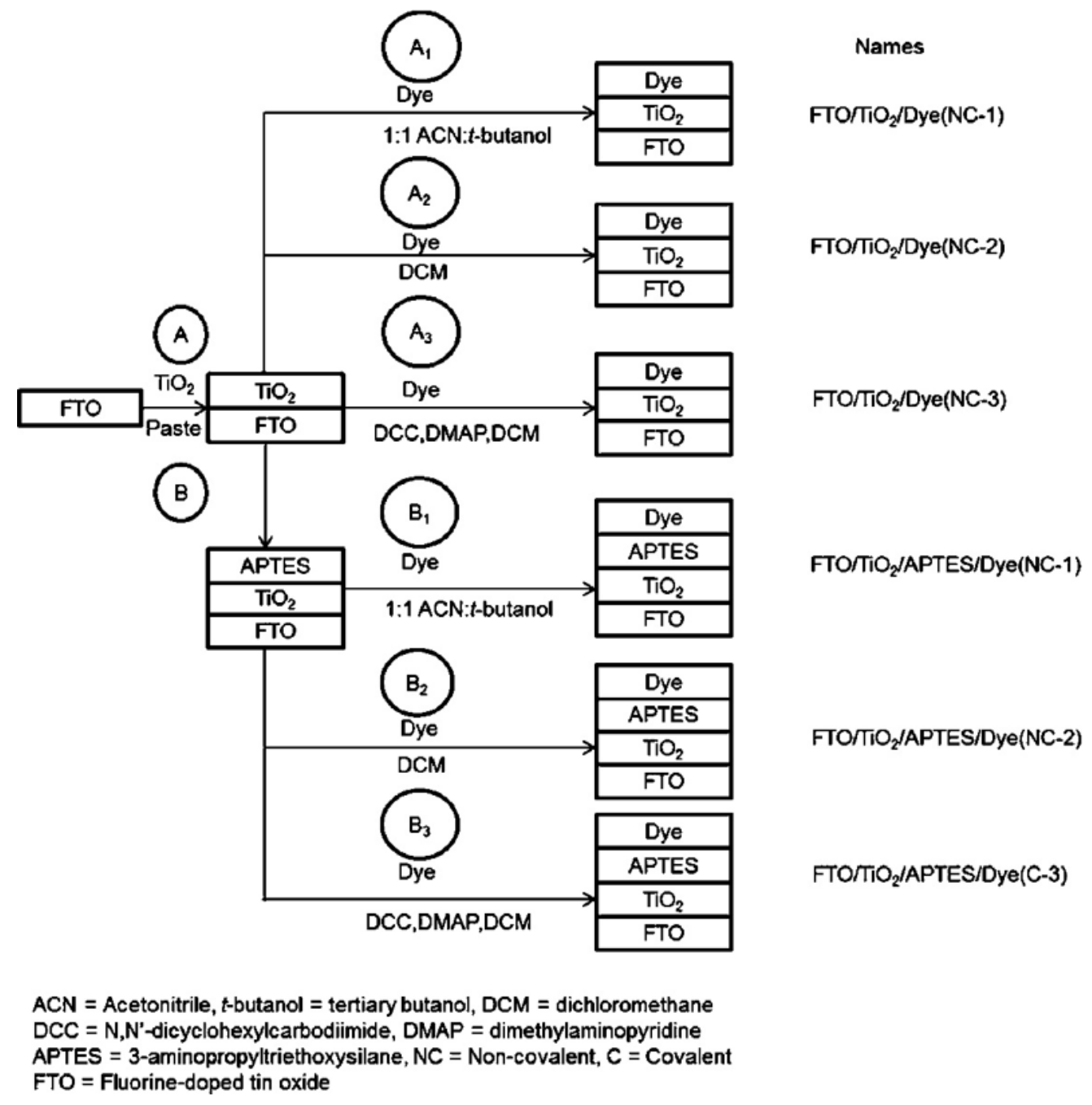

(C-3), where NC indicates "non-covalent" attachment, C indicates "covalent" attachment, and the number represents the solution used. We expected covalent attachment to occur in solution 3 by DCC and DMAP catalyzed amide bond formation between the amine groups of APTES and the carboxylate groups of N719 dye. 
While Scheme 3.1 illustrates the possible interactions between dye and $\mathrm{FTO} / \mathrm{TiO}_{2}$, Scheme 3.3 illustrates the different possible non-covalent interactions between APTESfunctionalized $\mathrm{TiO}_{2}\left(\mathrm{FTO} / \mathrm{TiO}_{2} /\right.$ APTES$)$ and $\mathrm{N} 719$ dye molecules, which includes $\mathrm{H}-$ bonding and electrostatic forces. We expected these types of interactions for

Scheme 3.3. Possible non-covalent interactions between dye and $\mathrm{FTO} / \mathrm{TiO}_{2} / \mathrm{APTES}$.
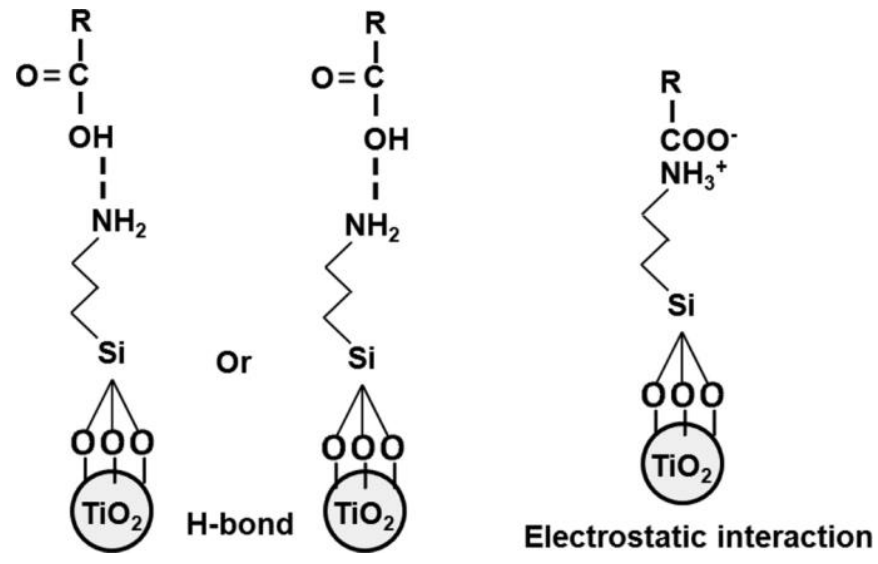

Scheme 3.4. Possible covalent interactions between dye and a) $\mathrm{FTO} / \mathrm{TiO}_{2} / \mathrm{APTES}$, b) $\mathrm{FTO} / \mathrm{TiO}_{2}$ when dye molecules are attached from a solution containing DCC and DMAP.

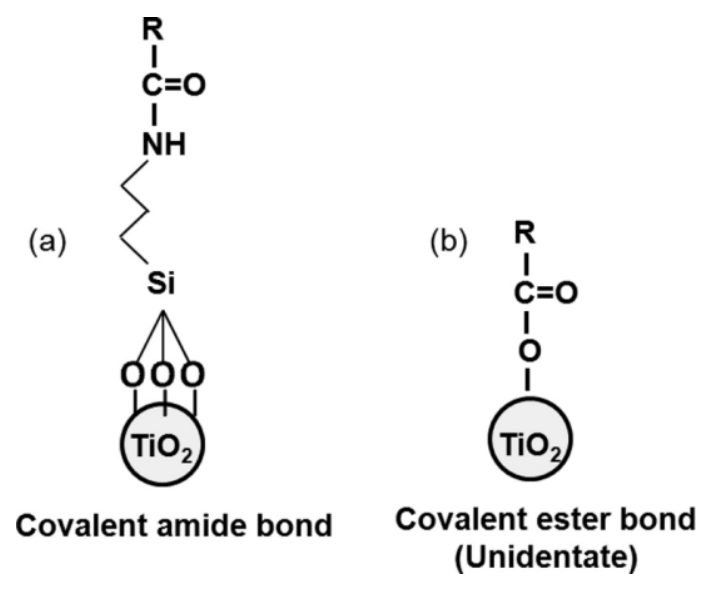

FTO/TiO 2 /APTES (NC-1) and FTO/TiO 2 /APTES (NC-2). Scheme 3.4 illustrates the possible covalent interactions between $\mathrm{FTO} / \mathrm{TiO}_{2} / \mathrm{APTES}$ and $\mathrm{FTO} / \mathrm{TiO}_{2}$ and dye in solution 3 to form covalent amide and ester bonds, respectively (steps A3 and B3). A covalent type of ester bond could form in step A3, which is also known as the unidentate bonding 
described in Scheme 3.1. Since we could not confirm this and the behavior is very different from the covalent $\mathrm{FTO} / \mathrm{TiO}_{2} / \mathrm{APTES}$ (C-3) electrode as shown later, we label this PA as NC.

Photoactivity Measurements: All photoactivity measurements were from PAs prepared from $\mathrm{P} 25$ Degussa $\mathrm{TiO}_{2}$ nano-powder. Figure 3.3A shows current-voltage curves in the presence of solar simulated light of the six different DSSCs with the PAs

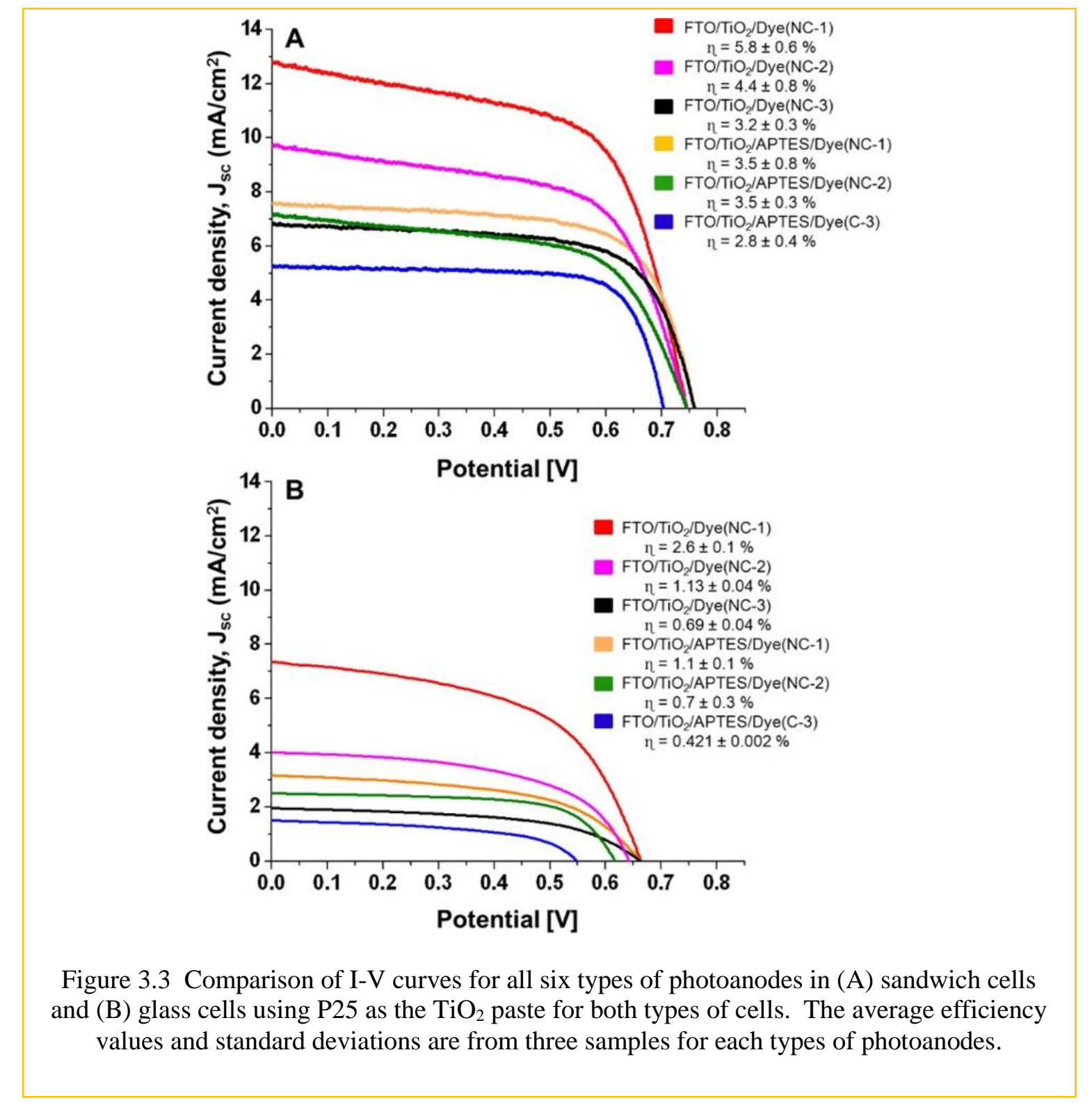

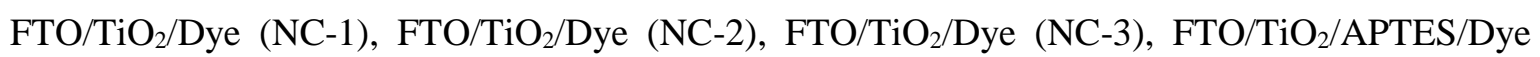

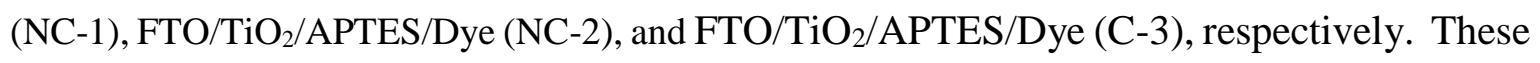


were measured in sandwich cells using Iodolyte 50 electrolyte (with $\mathrm{I}^{-} / \mathrm{I}_{3}{ }^{-}$) redox couple and an FTO/Pt counter electrode. PAs prepared by the traditional method of direct immersion in N719 dye using 1:1 acetonitrile:t-butanol as the solvent had an efficiency of $5.8 \pm 0.6 \%$. This is consistent with other reports using $\mathrm{P}_{25} \mathrm{TiO}_{2}$ and serves as our baseline value for comparison between the functionalized PAs and PAs prepared by the traditional approach. Attachment of $\mathrm{N} 719$ to $\mathrm{FTO} / \mathrm{TiO}_{2}$ by direct immersion from a dichloromethane solution without or with DCC and DMAP led to a decrease in efficiency to $4.4 \pm 0.8 \%$ and $3.2 \pm 0.3 \%$, respectively. This shows that the solvent can play a role in the efficiency and that the presence of DCC and DMAP (base) somehow altered the attachment chemistry or coverage of $\mathrm{N} 719$ to the $\mathrm{TiO}_{2}$ surface, which lowered the efficiency.

Surface modification of the $\mathrm{TiO}_{2}$ with APTES prior to dye adsorption from a 1:1 acetonitrile:t-butanol solution led to no significant change in the open circuit voltage (OCV) compared to the traditional approach, but resulted in a lower photocurrent and lower efficiency. The efficiency was $3.5 \pm 0.8 \%$ and $3.5 \pm 0.3 \%$, respectively for N719 attachment in 1:1 acetonitrile:t-butanol and dichloromethane, respectively. In this case, the solvent did not make a difference, but both showed lower efficiency compared to N719 attached to bare $\mathrm{FTO} / \mathrm{TiO}_{2}$. The efficiency of the $\mathrm{FTO} / \mathrm{TiO}_{2} / \mathrm{APTES} / \mathrm{Dye}(\mathrm{C}-3)$ was the lowest at $2.8 \pm 0.4 \%$. In this case, dye is expected to be attached through a covalent amide bond.

Clearly both solvent and functionalization (surface chemistry) can affect the efficiency of the PAs in the DSSCs. The trend is that attachment of dye from solution 2 and 3 led to lower efficiency when compared to solution 1 for bare $\mathrm{FTO} / \mathrm{TiO}_{2} \mathrm{PAs}$. Also, dye attached to functionalized $\mathrm{FTO} / \mathrm{TiO}_{2} / \mathrm{APTES}$ PAs showed lower efficiency compared to direct attachment of dye in all 3 solutions. The type of solvent and presence of base (DMAP) 
may lead to a different coverage and/or a different type of interaction with the $\mathrm{TiO}_{2}$ surface. The presence of APTES on $\mathrm{TiO}_{2}$ could affect electronic coupling between the dye and the $\mathrm{TiO}_{2}$ surface, which would affect electron injection from the excited state of the dye to the conduction band of $\mathrm{TiO}_{2}$. If electron injection is hindered, this could lower the efficiency, which would not be too surprising since there is an insulating organic layer between the $\mathrm{TiO}_{2}$ and dye when APTES is present.

Stability: It is known that PAs based on N719 dye are not stable indefinitely. ${ }^{35,92,96,102}$ Exposure to air, water, UV light, heat, and other chemicals can lead to degradation over time. ${ }^{55,94}$ For this reason we decided to first test the efficiency of the 6 PAs prepared after exposure to air for up to 60 days. We were not able to use sealed sandwich cells for this experiment, because it would require either 1) using a new PA for each air exposure time or 2) removing the PAs from the sealed cell, exposing to air for a certain amount of time, and then testing again in a sealed cell. For convenience, we instead used a glass cell open to air. Figure 3.3B shows the current-voltage curves of the 6 PAs using glass cells and solar simulated light. They all have lower values of photocurrent (and efficiency) when compared to the sandwich cell set up, but the trends in the efficiency are basically the same. These 6 PAs were tested in the glass cell directly after they were made and then again after sitting in air for various times up to 60 days.

Figure 3.4 shows a plot of the efficiency of the 6 PAs as a function of the number of days exposed to air over the 60-day time period. The same PA was tested over the 60 days. This was performed 3 times for each type of PA and Figure 3.4 shows the average efficiency plus standard deviation. Interestingly, all 5 PAs with dye attached "noncovalently" showed a significant loss in photoactivity after 10 days, where they became 
lower than the efficiency of the PA with covalently-attached dye. Also, they showed a complete loss in photoactivity between 20-30 days. In contrast, the efficiency of DSSCs with covalently-attached dye actually increased slightly during the 60 day time period and was stable well beyond that. We believe the slight increase in efficiency may be due to

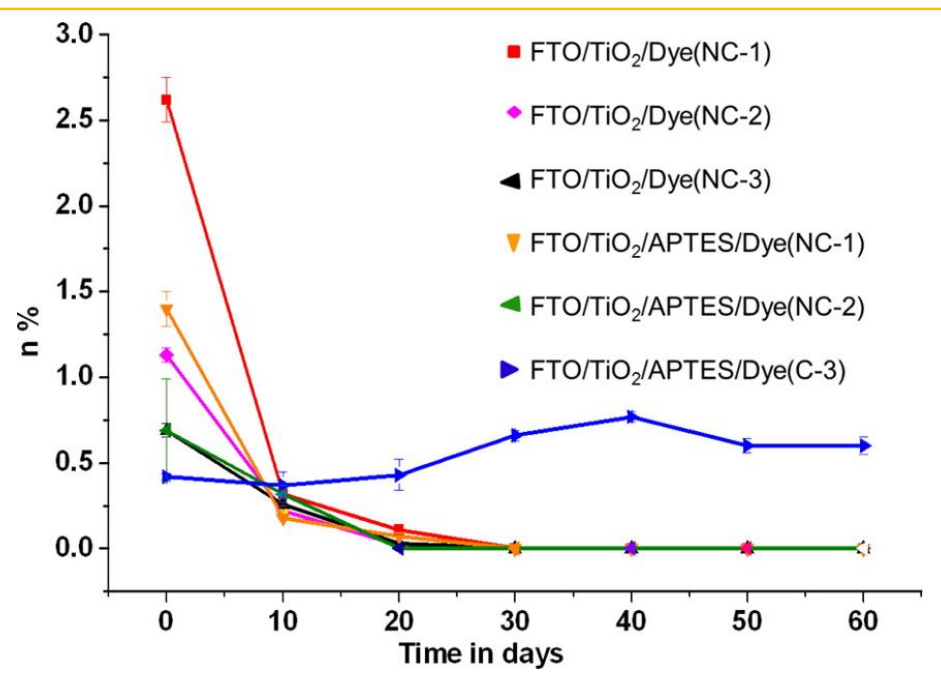

Figure 3.4. The efficiency of DSSCs prepared with the indicated photoanodes using the glass cell set-up as a function of photoanode exposure time in air. The $\mathrm{TiO}_{2}$ paste was $\mathrm{P} 25$.

some loss of N719 dye multilayers that initially exists on the PA. Multilayers of N719 dye can lead to lower efficiency. While the efficiency was lower initially for the covalentlyattached dye, the stability was significantly better compared to the traditionally-attached dye and other non-covalent PAs. This would allow the long term storage in air without degradation of performance. In addition, it is very likely that the stability would also be superior in the sealed sandwich cells over long periods of time. These could be important practical advantages that could benefit DSSC technology.

Spectroscopy Measurements: In order to better understand the nature of the interaction and compare the coverage between the dye and the $\mathrm{FTO} / \mathrm{TiO}_{2}$ or FTO/TiO 2 /APTES, we performed ATR-FTIR and UV-vis characterization of the 6 different 
PAs. Figure 3.5 shows the results of ATR-FTIR spectroscopy of the N719 dye adsorbed on the different PAs. In all spectra, the peaks at 1604 and $1375 \mathrm{~cm}^{-1}$ correspond to the

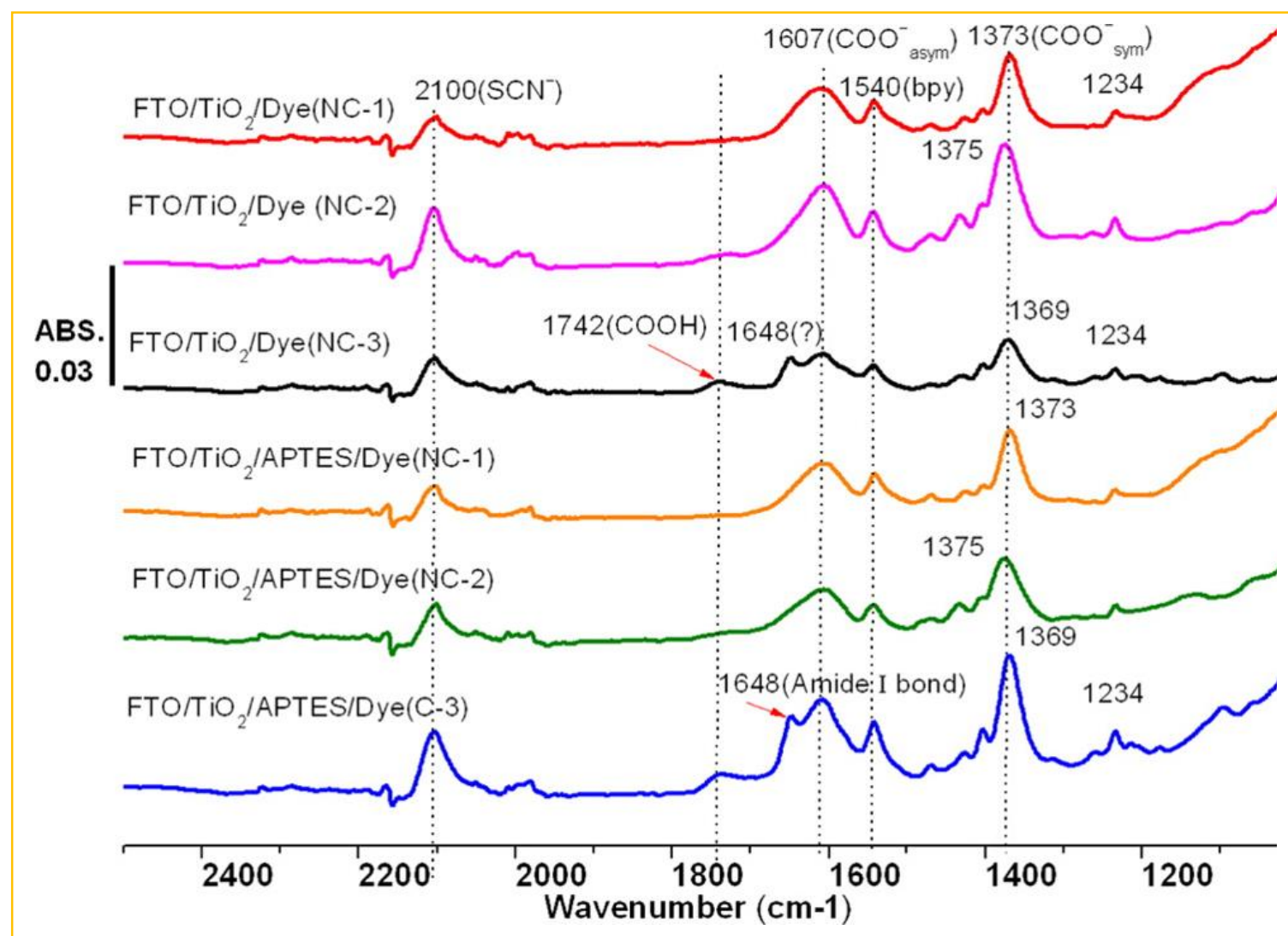

Figure 3.5. ATR-FTIR spectroscopy of the six different photoanodes prepared in this study using Dyesol $\mathrm{TiO}_{2}$ paste.

asymmetric and symmetric stretch of the carboxylate group, respectively. The peak at 1542 $\mathrm{cm}^{-1}$ corresponds to the bipyridyl group and the peak at $2109 \mathrm{~cm}^{-1}$ is due to the $\mathrm{CN}$ stretch from the two SCN ligands on each N719 dye molecule. This confirms dye attachment to the PAs in all cases. The difference between the asymmetric and symmetric stretch of the carboxylate groups is smaller than that of the bulk dye, which indicates involvement of the carboxylate groups through the bridging mode and possibly hydrogen bonding in the attachment to $\mathrm{TiO}_{2}$ and $\mathrm{TiO}_{2} / \mathrm{APTES}$. The intensity of the $\mathrm{CN}$ stretch is indicative of the dye coverage. In this set of data, $\mathrm{FTO} / \mathrm{TiO}_{2} / \mathrm{Dye}(\mathrm{NC}-2)$ and $\mathrm{FTO} / \mathrm{TiO}_{2} / \mathrm{APTES} / \mathrm{Dye}(\mathrm{C}-3)$ show 
the highest CN stretch intensities, and therefore dye coverage, but the values are somewhat variable from sample-to-sample. Table 3.1 shows the average absorbance of the $\mathrm{CN}$ stretch with standard deviation from the ATR-FTIR data for all 6 PAs studied. While there is significant variability, we generally observed that the coverage was larger for dye adsorbed from dichloromethane (solutions 2 and 3) than from the acetonitrile:t-butanol mixture (solution 1). The high coverage may be due to the formation of multilayers of N719 dye because of lower solubility in dichloromethane. This could partly cause the lower efficiencies observed for PAs prepared from solutions 2 and 3

The ATR-FTIR data in Figure 3.5 confirm dye attachment and give some idea of coverage, but also confirm the covalent amide attachment of dye in the FTO/TiO 2 /APTES/Dye (C-3) sample by the presence of a peak at $1648 \mathrm{~cm}^{-1}$, which

Table 3.1. ATR-FTIR and UV-vis absorbance values of the various photoanodes prepared in this study. The absorbance values are related to the dye coverage.

\begin{tabular}{|c|c|c|c|}
\hline Photoanodes & $\begin{array}{c}\text { ATR-FTIR ABS. } \\
\text { peak height } \\
\left(\mathrm{SCN}^{-}\right) \\
\end{array}$ & $\begin{array}{c}\text { ATR-FTIR ABS. } \\
\text { peak height } \\
\left(\mathrm{SCN}^{-}\right) \\
\end{array}$ & $\begin{array}{l}\text { UV-vis ABS at } \\
\lambda=520-545 \mathrm{~nm}\end{array}$ \\
\hline & P25 & DSL & DSL \\
\hline FTO/TiO $/$ Dye(NC-1) & $\begin{array}{c}0.0072 \pm 0.0033 \\
(\mathrm{~N}=12)\end{array}$ & $\begin{array}{c}0.0067 \pm 0.0015 \\
(\mathrm{~N}=13)\end{array}$ & $\begin{array}{c}0.508 \pm 0.198 \\
(\mathrm{~N}=36)\end{array}$ \\
\hline FTO/TiO $/$ Dye(NC-2) & $\begin{array}{c}0.0103 \pm 0.0090 \\
(\mathrm{~N}=9)\end{array}$ & $\begin{array}{c}0.0082 \pm 0.0028 \\
(\mathrm{~N}=4)\end{array}$ & $\begin{array}{c}0.706 \pm 0.096 \\
(\mathrm{~N}=7)\end{array}$ \\
\hline FTO/TiO $/$ Dye(NC-3) & $\begin{array}{c}0.0122 \pm 0.0018 \\
(\mathrm{~N}=8)\end{array}$ & $\begin{array}{c}0.0090 \pm 0.0040 \\
(\mathrm{~N}=15)\end{array}$ & $\begin{array}{c}0.717 \pm 0.303 \\
(\mathrm{~N}=24)\end{array}$ \\
\hline FTO/TiO $2 /$ APTES/Dye(NC-1) & $\begin{array}{c}0.0053 \pm 0.0021 \\
(\mathrm{~N}=7)\end{array}$ & $\begin{array}{c}0.0058 \pm 0.0019 \\
(\mathrm{~N}=15)\end{array}$ & $\begin{array}{c}0.462 \pm 0.120 \\
(\mathrm{~N}=32)\end{array}$ \\
\hline FTO/TiO $2 / \mathrm{APTES}_{2} \mathrm{Dye}(\mathrm{NC}-2)$ & $\begin{array}{c}0.0071 \pm 0.0029 \\
(\mathrm{~N}=8)\end{array}$ & $\begin{array}{c}0.0056 \pm 0.0029 \\
(\mathrm{~N}=4)\end{array}$ & $\begin{array}{c}0.655 \pm 0.144 \\
(\mathrm{~N}=6)\end{array}$ \\
\hline 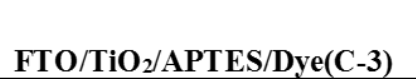 & $\begin{array}{c}0.0142 \pm 0.0097 \\
(\mathrm{~N}=17)\end{array}$ & $\begin{array}{c}0.0100 \pm 0.0032 \\
(\mathrm{~N}=13)\end{array}$ & $\begin{array}{c}0.975 \pm 0.333 \\
(\mathrm{~N}=35)\end{array}$ \\
\hline
\end{tabular}

$\mathrm{N}=$ the number of samples studied; P25 = P25 DegussaTiO 2 ; DSL $=$ Dyesol $\mathrm{TiO}_{2}$

corresponds to the amide $\mathrm{I}(\mathrm{C}=\mathrm{O})$ stretch. To confirm this assignment, we reacted benzoic 
acid with $\mathrm{FTO} / \mathrm{TiO}_{2} / \mathrm{APTES}$ in the same way. In that case, with only one carboxylic acid group on benzoic acid, there were no carboxylate peaks present and only one well-defined peak at $1646 \mathrm{~cm}^{-1}$ corresponding to the amide I bond found in Figure 3.6. This confirms our peak assignment. The spectrum of $\mathrm{FTO} / \mathrm{TiO}_{2} / \mathrm{APTES} / \mathrm{Dye}(\mathrm{C}-3)$ contains a significant amount of unreacted carboxylate groups, showing that some of the carboxylates on the dye

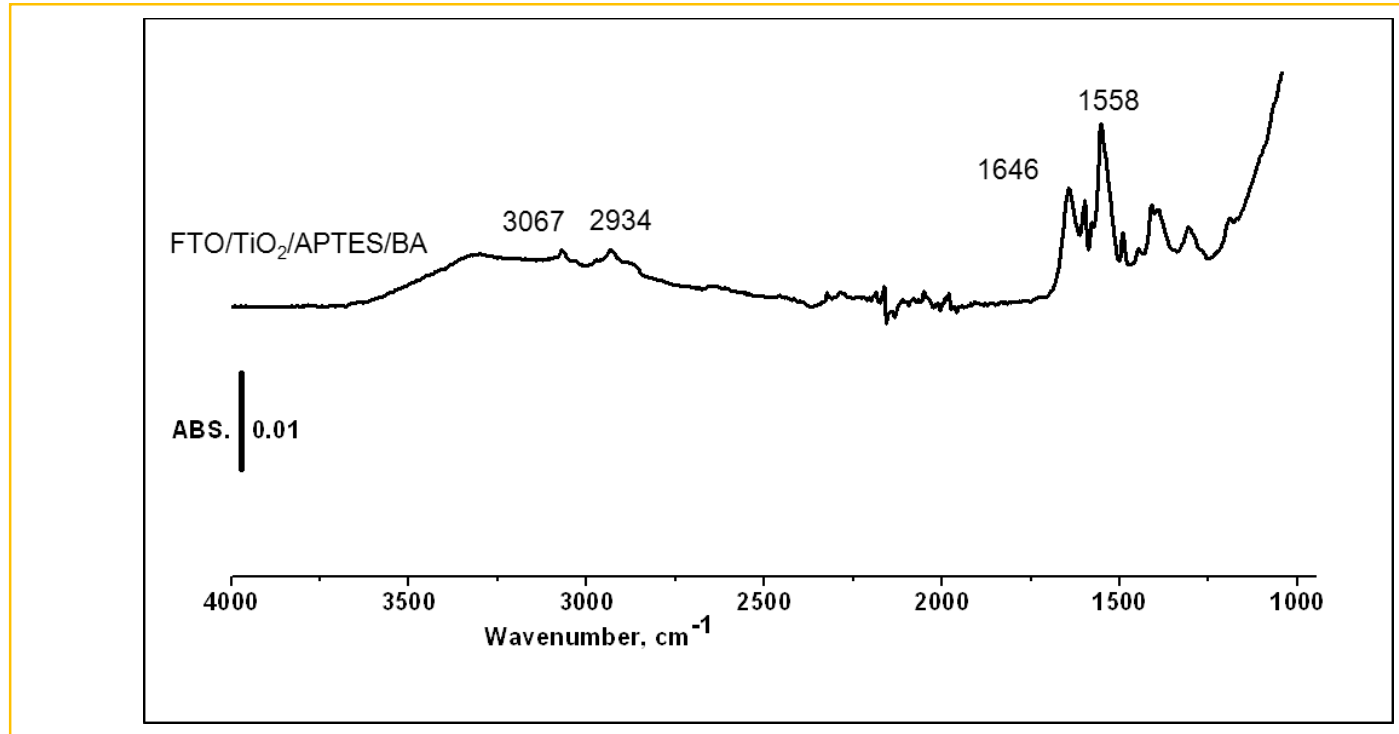

Figure 3.6. ATR-FTIR spectrum of benzoic acid (BA) attached to FTO/TiO $/ 2$ APTES from a dichloromethane solution containing DCC and DMAP. The peak at $1646 \mathrm{~cm}^{-1}$ is consistent with an amide I stretch, confirming covalent amide bond attachment. This peak is similar to the covalent amide bond stretch observed for $\mathrm{N} 719$ dye attached to $\mathrm{FTO} / \mathrm{TiO}_{2} / \mathrm{APTES}$ in a similar manner.

do not attach covalently through an amide and there may also be a partial multilayer or aggregates of N719 dye on top of the covalently bound dye. It is possible that dye multilayers contributed to the lower efficiency shown in Figure 3.4. The covalently bound dye consistently showed the highest coverages in the ATR-FTIR and UV-vis data (Table 3.1).

Figure 3.5 shows that the $\mathrm{FTO} / \mathrm{TiO}_{2} / \mathrm{Dye}$ (NC-3) PA also exhibits a peak near 1648 $\mathrm{cm}^{-1}$, even though amide bond formation is not possible in this case. This was puzzling, since we confirmed that this peak is in the range of the amide I stretch as was confirmed 
with the benzoic acid experiment. Since the amide bond is not possible with this PA, we believe that this peak is due to the $\mathrm{C}=\mathrm{O}$ stretch from a carboxylate in either the unidentate configuration (ester bond) or some other bonding form on $\mathrm{TiO}_{2}$. This type of bonding could be favored in the presence of DCC and DMAP since they also can catalyze ester bond formation between $\mathrm{COOH} / \mathrm{COO}^{-}$and $\mathrm{OH}$ groups. While a $\mathrm{CO}-\mathrm{OR}$ type ester bond is normally near $1720 \mathrm{~cm}^{-1}$, the CO-O-Ti ester bond may be at lower wavenumber due to a more stable resonance structure that gives the $\mathrm{C}=\mathrm{O}$ more single bond character. The resonance structure that gives the $\mathrm{C}=\mathrm{O}$ single bond character places a positive charge on the $\mathrm{O}$ next to the Ti. This positive charge on $\mathrm{O}$ may be more favored when next to $\mathrm{Ti}$ as compared to when next to an $\mathrm{R}$ group, which lowers the $\mathrm{C}=\mathrm{O}$ stretching frequency to a value similar to the amide I $\mathrm{C}=\mathrm{O}$ stretch. Interestingly, the $1648 \mathrm{~cm}^{-1}$ peak (Figure 3.7) was also present in a dichloromethane solution with DMAP, but not DCC, showing that the presence of the DMAP base alone was enough to favor this type of bonding. Others have assigned a peak at $1720-1742 \mathrm{~cm}^{-1}$ to unidentate, or ester bond formation. ${ }^{70}$ While we do not know this peak assignment conclusively, we know that it is promoted by base only (DMAP) and it is different from the amide $\mathrm{I}$ bonding $\mathrm{C}=\mathrm{O}$ stretch at a similar wavenumber. We later show that the chemistry of these two PAs is very different when soaking in acetic acid (Figure 3.12), which gives further evidence that the origin of the peak at $1648 \mathrm{~cm}^{-1}$ is different for the two PAs.

Figure 3.8 shows the UV-vis spectra of all six PAs. N719 dye in solution has three peaks, two of which correspond to metal-to-ligand charge transfer transitions (MLCT) 4d$\pi^{*}(520-545 \mathrm{~nm}$ and $380-395 \mathrm{~nm})$, and another at the shorter wavelength around $313 \mathrm{~nm}$, corresponding to a ligand-to-ligand charge transfer transition (LLCT) $\pi-\pi^{*}$ ). ${ }^{103}$ The MLCT 


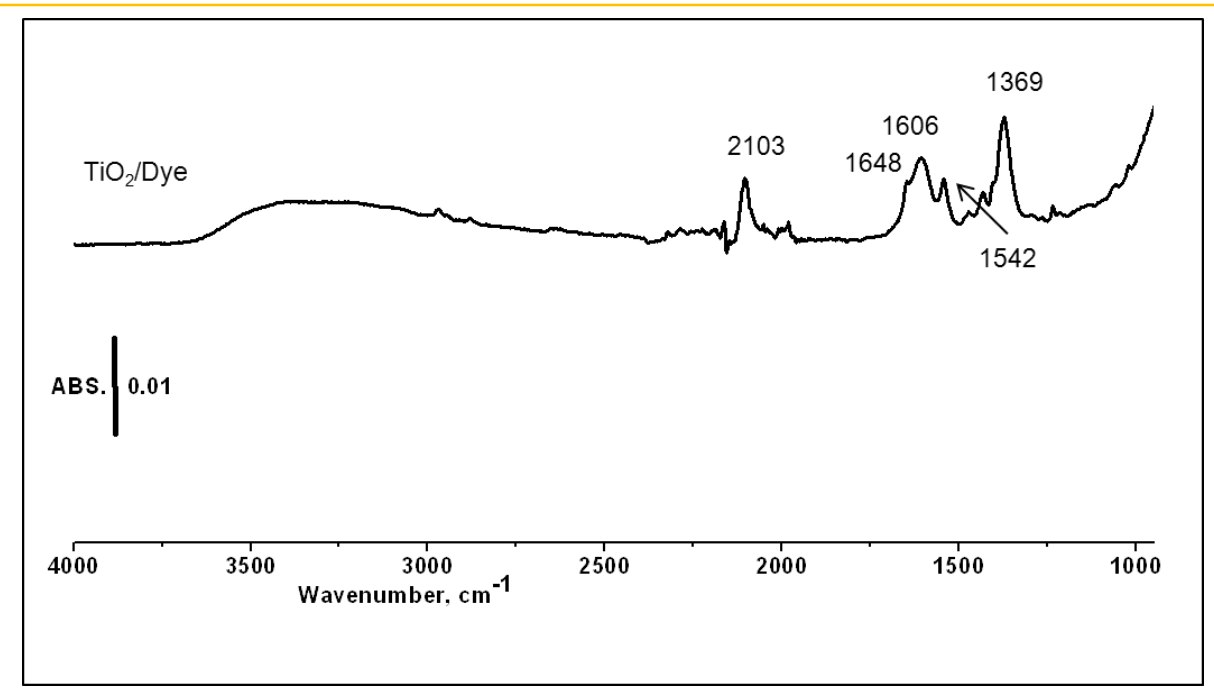

Figure 3.7. ATR-FTIR spectrum of N719 dye attached to $\mathrm{TiO}_{2}$ from a dichloromethane solution containing DMAP only (no DCC). The peak at $1648 \mathrm{~cm}^{-1}$ is still present, showing that DMAP alone can lead to the $\mathrm{TiO}_{2} /$ Dye interaction responsible for this peak.

peaks were blue-shifted for FTO/TiO $2 /$ APTES/Dye PAs, including the PA with covalentlyattached dye, relative to those for dye in solution. In contrast, the MLCT peaks were redshifted for $\mathrm{FTO} / \mathrm{TiO}_{2} /$ Dye PAs relative to dye in solution. The red shift is consistent with a

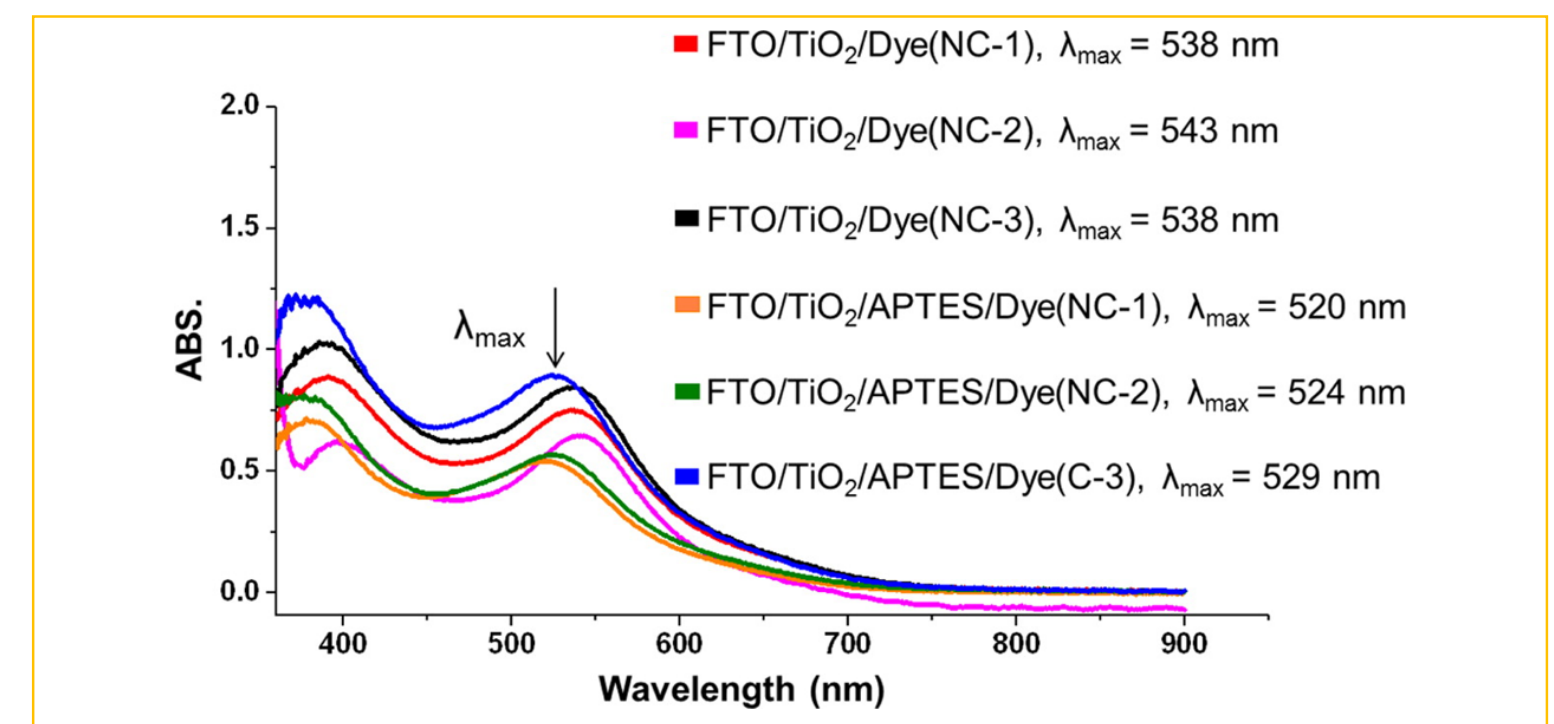

Figure 3.8. UV-vis spectroscopy of $\mathrm{N} 719$ dye adsorbed on Dyesol $\mathrm{TiO}_{2}$ paste for the six photoanodes.

${ }^{103}$ direct interaction with the $\mathrm{TiO}_{2}$ surface and the blue shift is consistent with adsorption through the APTES layer, leading to less direct coupling to $\mathrm{TiO}_{2}$. Table 3.1 shows the 
average UV-vis absorbance for the peak at 520-545 nm. Again, the PAs prepared from dichloromethane solution have the highest coverage of dye, which may indicate the formation of multilayers. Of the two dichloromethane solutions, the solution with DMAP and DCC generally had higher dye coverages. The covalent bonding, presence of base, and low solubility of dye in dichloromethane are the likely reasons. Low coverage is clearly not the reason for lower efficiency with these PAs. Instead, the formation of multilayers and poor coupling of the dye to the $\mathrm{TiO}_{2}$ are more likely. The traditionally-prepared dye has the highest efficiency and usually the lowest coverage since the acetonitrile/t-butanol solvent system is optimized for monolayer coverage, which is consistent with our results. The FTO/TiO 2 /APTES/Dye (NC-1) has the optimal solvent system, but the presence of the APTES lowered the coverage somewhat, which may have led to its slightly lower efficiency, along with poor coupling due to APTES. There appears to be trends with coverage and efficiency, but it is difficult to know the relative importance of coverage as compared to the dye coupling strength to the surface.

We obtained ATR-FTIR and UV-vis spectroscopy measurements before and after exposure to air in order to better understand the increased stability of the PAs with covalently-attached dye. With this data, we focus on the PA with covalently-attached dye compared to the traditionally-prepared dye. Figure 3.9A shows the ATR-FTIR data of FTO/TiO 2 /Dye (NC-1) and $\mathrm{FTO}_{\text {TiO }} / \mathrm{APTES}_{\text {D }}$ ye (C-3) before and after exposure to air for 20 days. There are clear differences in the before and after spectra for the traditional PA as compared to the PA with covalently-attached dye. For the traditional PA, the peaks corresponding to the symmetric and asymmetric stretch of the carboxylate groups and the bipyridyl peak all decrease after 20 days in air. Most notably, though, the $\mathrm{CN}$ stretch of the 
NCS ligand is completely gone after 20 days. For the PA with covalently-attached dye, the carboxylate and bipyridyl peaks also decrease in intensity, but not nearly as much. Also, the amide peak at $1648 \mathrm{~cm}^{-1}$ appears to have decreased less in comparison to the carboxylate and bipyridyl peak. Interestingly, the $\mathrm{CN}$ stretch due to NCS has decreased a little, but is still significant.
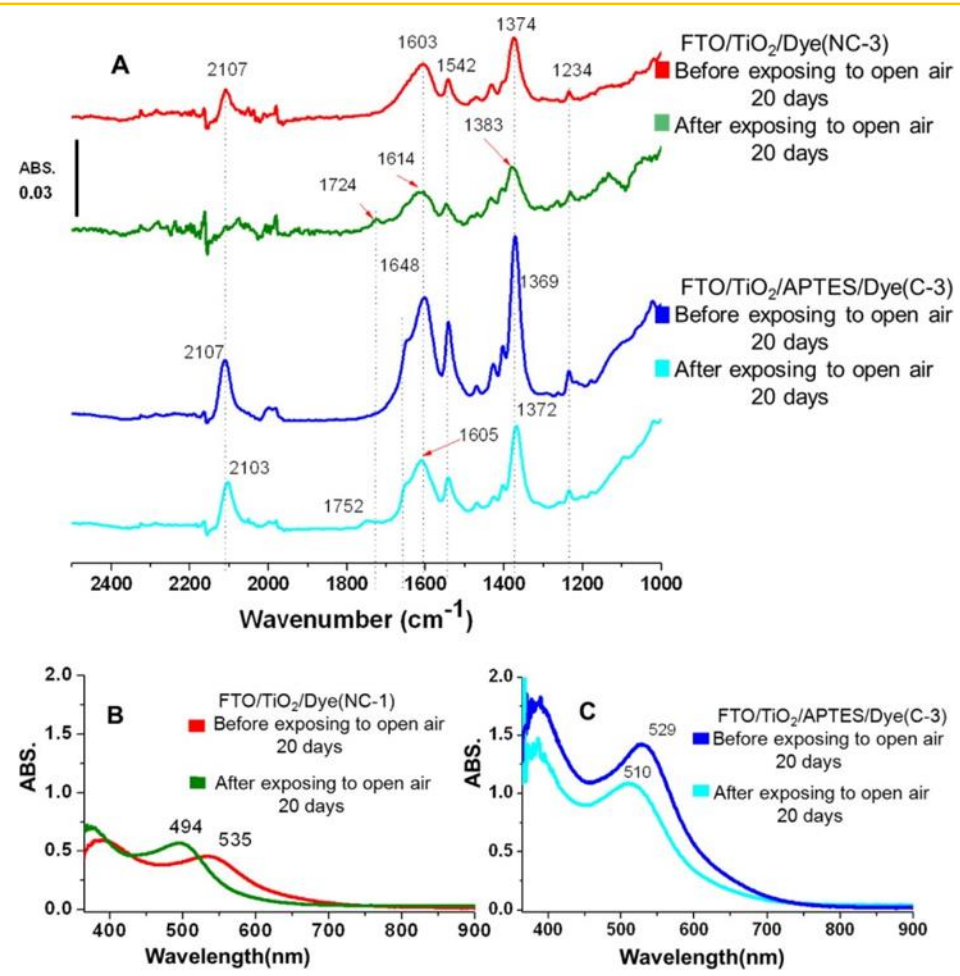

Figure 3.9. Air stability test. (A) Top: ATR-FTIR spectra of conventional FTO/ $\mathrm{TiO}_{2} / \mathrm{Dye}$ (NC1), red 1st day and green after 20 days. Below: ATR-FTIR of covalent FTO/TiO $2 / \mathrm{APTES} / \mathrm{Dye}$ (C-3), blue $1^{\text {st }}$ day and cyan after 20 days. (B) UV-vis spectra of conventional $\mathrm{FTO} / \mathrm{TiO}_{2} / \mathrm{Dye}$ (NC-1), red 1 st day and green after 20 days. (C) UV-vis of covalent FTO/TiO $/$ /APTES/Dye (C3 ), blue $1^{\text {st }}$ day and cyan after 20 days. Photoanodes were stored in a closed box filled with air.

Figure 3.9B and 3.9C shows the UV-vis data of the traditional PA and PA with covalently-bound dye, respectively. The initial absorbance, and therefore coverage, of the traditional dye is smaller than that of the covalently-bound dye, which is consistent with the statistics in Table 3.1. More importantly, the traditionally-attached dye absorbance peak at $535 \mathrm{~nm}$ shifted to $494 \mathrm{~nm}$ (41 nm shift) after air exposure for 20 days. In contrast, the 
absorbance band for the covalently-bound dye shifted from 529 to $510 \mathrm{~nm}$, which is only 19 nm.

Taken together, the ATR-FTIR and UV-vis data suggest that the loss in efficiency of the PA with dye attached in the traditional manner following air exposure is related to the loss in the NCS ligands from the N719 dye. This leads to complete loss of the CN stretch in the ATR-FTIR spectrum and a large $41 \mathrm{~nm}$ blue shift in the visible absorbance peak. The NCS ligand remained on the dye for 20 days (and likely longer based on efficiency data) when the dye was covalently-attached to the FTO/TiO 2 /APTES PAs as shown by the CN stretch remaining in the ATR-FTIR spectrum and the relatively small blue shift in the UVvis spectrum. This led to stable PA efficiency over the 60 days as shown in Figure 3.4. It is very interesting that the covalent amide bonding of dye to FTO/ $\mathrm{TiO}_{2} / \mathrm{APTES}$ leads to more stable NCS ligands since the ligands are not directly involved in the bonding. Hagfeldt and co-workers showed that the NCS ligands of traditional dye can be replaced by atmospheric $\mathrm{H}_{2} \mathrm{O}$ or $\mathrm{OH}$ when assembled on $\mathrm{TiO}_{2}$, which leads to poor air stability. ${ }^{104}$ Accordingly, we believe that covalent linkage somehow provides an environment that protects the NCS ligands from significant replacement by water in air, leading to long term air stability. It is not clear if the presence of the polymeric silane linker is directly responsible, if the molecule orientation is different after covalent linkage, or if some other mechanism provides stability. More experiments in the future are needed to better understand the details of the enhanced stability, but it is remarkable.

The photographs in Figure 3.10 show the different PAs with non-covalent attached and covalent attached dye (as indicated) before and after exposure to air for 20 days. The three PAs with non-covalent attached dye in $\mathrm{A}, \mathrm{B}$, and $\mathrm{C}$ changed from a dark red to a light 
orange color over the 20 day period, while the PA with covalent-attached dye (Picture D) remained the original dark red color over the entire 20 days. The color change from red to

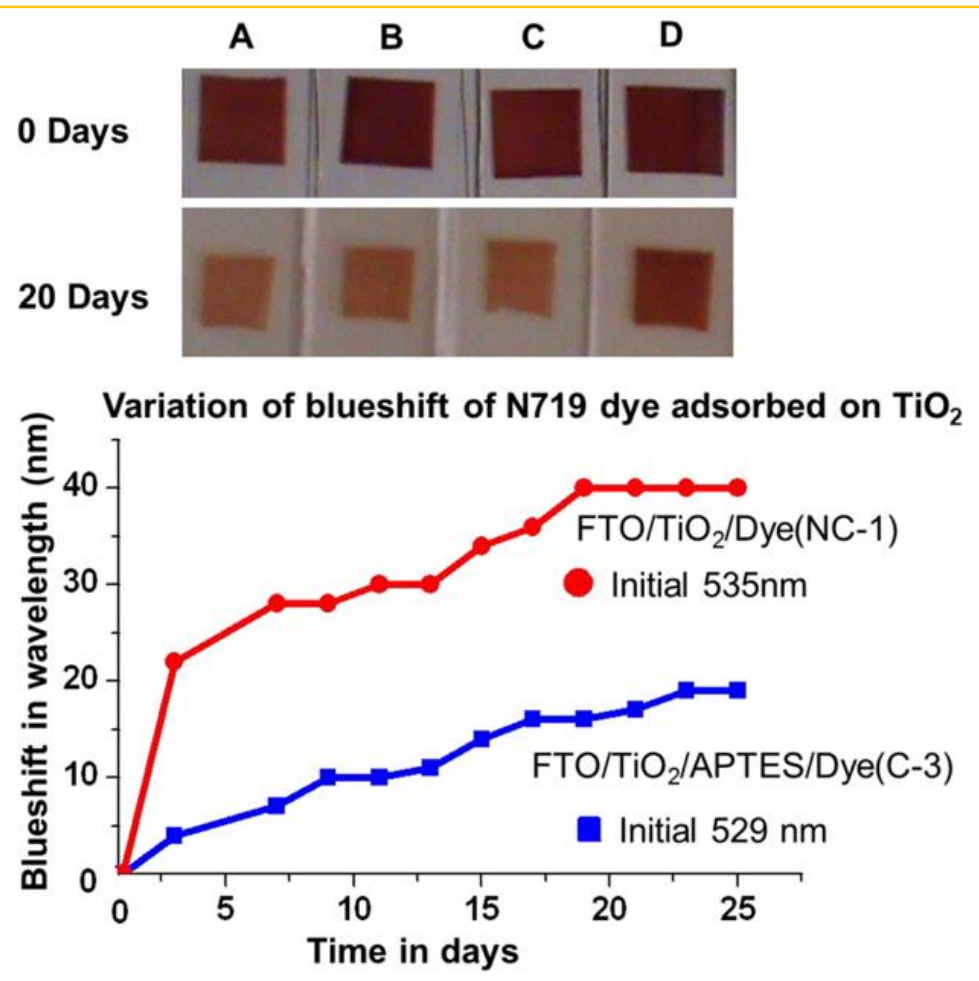

Figure 3.10. Photograph (A) $\mathrm{FTO} / \mathrm{TiO}_{2} / \mathrm{Dye}(\mathrm{NC}-1)$, (B) $\mathrm{FTO} / \mathrm{TiO}_{2} / \mathrm{Dye}$ (NC-2), (C) $\mathrm{FTO} / \mathrm{TiO}_{2} /$ Dye (NC-3), and (D) FTO/TiO $/$ APTES/Dye (C-3) before (0 days) and after 20 days of storage in open air. The plot below shows a graph of the blue shift of the MLCT band around 525-545 nm for $\mathrm{N} 719$ dye adsorbed on $\mathrm{TiO}_{2}$ as a function of storage time in air for the $\mathrm{FTO} / \mathrm{TiO}_{2} / \mathrm{Dye}(\mathrm{NC}-1)$ "red" and $\mathrm{FTO} / \mathrm{TiO}_{2} / \mathrm{APTES} / \mathrm{Dye}(\mathrm{C}-3)$ "blue" photoanodes.

orange is consistent with the ATR-FTIR and UV-vis data, and we believe it is due to the exchange of the thiocyanato ligand on the dye with atmospheric $\mathrm{H}_{2} \mathrm{O} / \mathrm{OH}^{-}$. This exchange can be accelerated by the presence of room light. A plot of the magnitude of the blue shift of the MLCT band of the dye adsorbed on $\mathrm{TiO}_{2}$ non-covalently and covalently over time of exposure to open air is also shown in Figure 3.10. The rate of exchange is much faster on the conventional PAs compared to the PAs with covalently-linked dye, which is responsible for the fast loss in efficiency with air exposure for the former. 
Figure 3.11A shows the ATR-FTIR spectra of the traditionally-prepared $\mathrm{FTO} / \mathrm{TiO}_{2} / \mathrm{Dye}(\mathrm{NC}-1)$ PA before and after heating at $140{ }^{\circ} \mathrm{C}$ with UV exposure for 3 hours. All of the peaks associated with the dye are reduced in intensity and the $\mathrm{CN}$ stretch from the
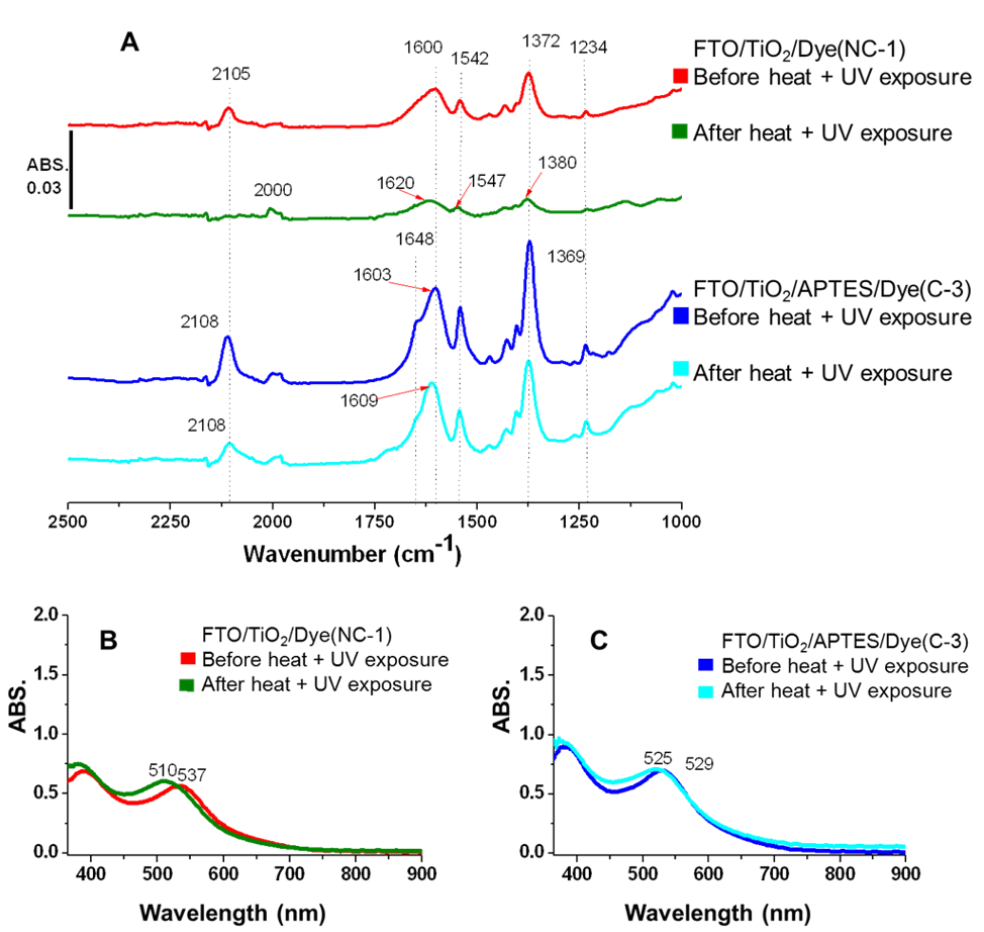

Figure 3.11. (A) ATR-FTIR spectra of conventional $\mathrm{FTO} / \mathrm{TiO}_{2} / \mathrm{Dye} /(\mathrm{NC}-1)$ and covalent $\mathrm{FTO} / \mathrm{TiO}_{2} / \mathrm{APTES} / \mathrm{Dye}(\mathrm{C}-3)$ photoanodes before and after heating in open air at $140{ }^{\circ} \mathrm{C}$ for $3 \mathrm{~h}$ and simultaneously illuminating with UV light. (B) and (C) are UV-vis spectra of conventional

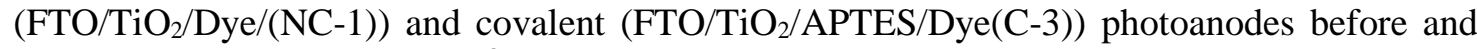
after heating in open air at $140{ }^{\circ} \mathrm{C}$ for $3 \mathrm{~h}$ and simulataneouly exposing to UV light. The samples were exposed to both $254 \mathrm{~nm}$ and $364 \mathrm{~nm}$ UV-light.

NCS ligand is almost completely absent after the heat and UV exposure. The UV-vis spectrum of the dye molecules of the same sample in Figure 3.11B shows a $27 \mathrm{~nm}$ blue shift of the MLCT band after heat and UV exposure, which is consistent with the loss of the NCS ligand in the ATR-FTIR spectrum. Figure 3.11C shows the ATR-FTIR spectra of the FTO/TiO 2 /APTES/Dye (C-3) PA before and after the same $140{ }^{\circ} \mathrm{C}$ heat treatment with UV exposure for $3 \mathrm{~h}$. The peaks after treatment are also lower in intensity, but the $\mathrm{CN}$ stretch from the NCS ligand is much more pronounced. The UV-vis spectra before and after the 
same treatment on the same covalently-linked PA in Figure 3.11C accordingly shows a very small $4 \mathrm{~nm}$ blue shift in the MLCT band of the dye. This combined data shows that the covalent linkage of the dye to the $\mathrm{TiO}_{2}$ through the APTES linker provides the dye with much higher stability against thermal and UV degradation compared to the dye attached traditionally through direct adsorption to $\mathrm{TiO}_{2}$.

Figure 3.12A shows ATR-FTIR spectra of a covalent PA (FTO/TiO $2 / \mathrm{APTES}_{2}$ Dye (C-3)) and non-covalent ( $\left.\mathrm{FTO} / \mathrm{TiO}_{2} / \mathrm{Dye}(\mathrm{NC}-3)\right)$ respectively before and after soaking in a $28 \mathrm{mM}$ solution of acetic acid in acetonitrile for $12 \mathrm{~h} .{ }^{102}$ In Figure 3.12, we compared the PA with covalently-attached dye to the PA with dye attached directly in the dichloromethane solution with DCC and DMAP since they both had a similar peak at $1648 \mathrm{~cm}^{-1}$ and we wanted to show that the chemistry of these two PAs is very different. As shown earlier in Figure 3.5, the ATR-FTIR spectra are very similar before exposure to acetic acid. They both exhibit the CN, carboxylate, and bipyridyl peaks along with the peak near 1648--1651 $\mathrm{cm}^{-1}$. After exposure to acetic acid, the carboxylate, bipyridyl and $1648 \mathrm{~cm}^{-1}$ peaks all decrease dramatically for the non-covalent $\mathrm{FTO} / \mathrm{TiO}_{2} / \mathrm{Dye}$ (NC-3) PA and the $\mathrm{CN}$ stretch is completely gone. In contrast, the same peaks all decrease slightly for the covalent.

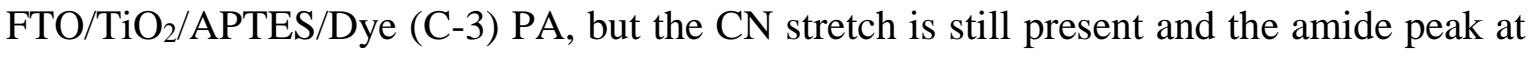
$1651 \mathrm{~cm}^{-1}$ remains very strong. While the carboxylates decrease, the amide peak remained relatively strong. This confirms that the nature of this peak is different from that of the $\mathrm{FTO} / \mathrm{TiO}_{2} /$ Dye (NC-3) PAs. The data suggests that it is a more stable bond, which is consistent with our assigned amide I bond. The loss of the carboxylates is likely due to removal of multilayers of N719 dye through protonation of the carboxylates by the acetic acid. Figure 3.12B shows a photograph of the covalent and non-covalent PAs before and 
after treatment with $28 \mathrm{mM}$ acetic acid solution in ethanol for $12 \mathrm{~h}$. The covalent PAs remained a dark red-purple color after treatment, while the non-covalent PAs became a light orange color due to dye degradation in the acid solution. The acid treatment experiments showed that the covalent attachment with APTES is indeed an amide bond and different

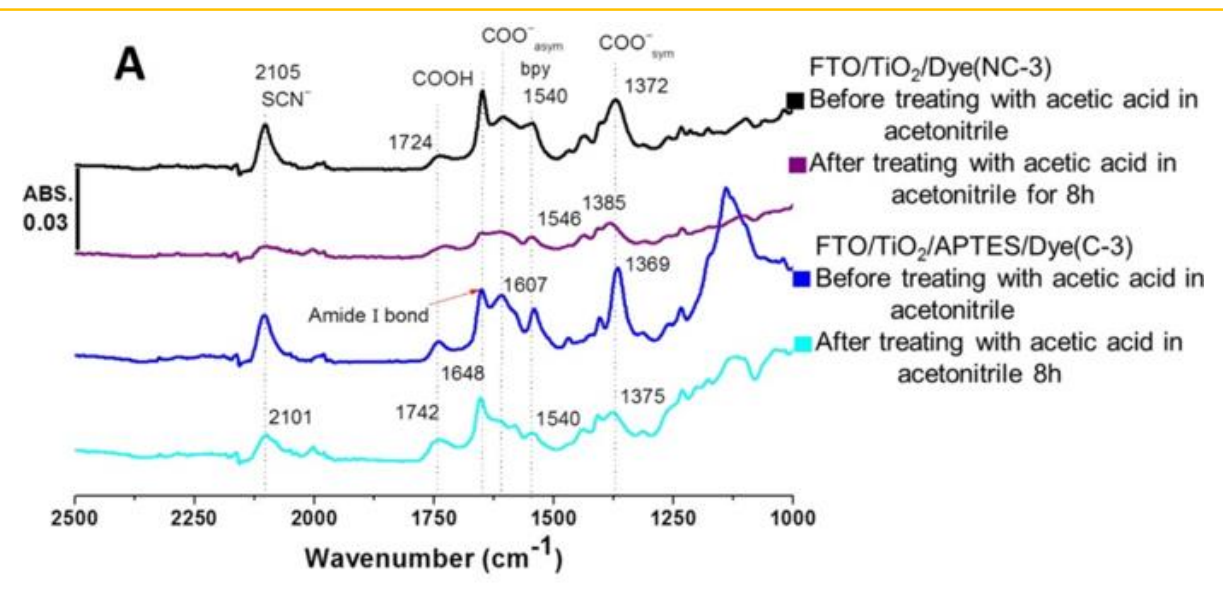

B

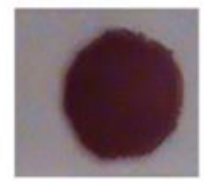

$28 \mathrm{mM}$ acetic acid solution in $\mathrm{CH}_{3} \mathrm{CN}$

$8 \mathrm{~h}$ at room temperature $\mathrm{FTO} / \mathrm{TiO}_{2} /$ Dye (NC-3)

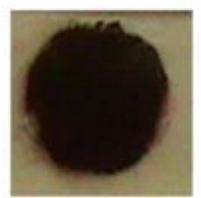

$28 \mathrm{mM}$ acetic acid solution in $\mathrm{CH}_{3} \mathrm{CN}$,

$8 \mathrm{~h}$ at room temperature FTO/TiO 2 /APTES/Dye (C-3)
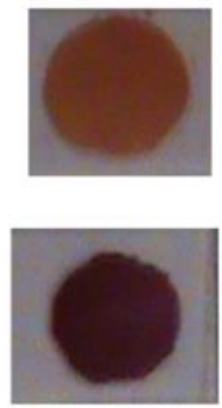

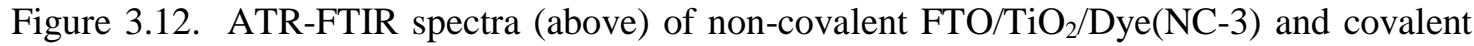
FTO/TiO 2 /APTES/Dye (C-3) photoanodes before and after soaking in $28 \mathrm{mM}$ solution of acetic acid in acetonitrile for $12 \mathrm{~h}$. The photographs show a non-covalent photoanode $\mathrm{FTO} / \mathrm{TiO}_{2} / \mathrm{Dye}$ (NC-3) (upper) and a covalent photoanode FTO/TiO $/$ APTES/Dye (C-3) (lower) before and after same acetic acid treatment. While the ATR-FTIR spectra are initially very similar, the chemistry of these two photoanodes is very different.

from the direct $\mathrm{TiO}_{2}$ attachment in the same solution. The PA with covalently-bound dye is clearly more stable to organic acids.

We also treated the PAs prepared traditionally in a pH 2.5 solution of acetic acid in ethanol for $15 \mathrm{~min}$ in comparison to the PAs with covalently-linked dye. The conventional PAs lost $65 \%$ of the adsorbed dye molecules based on the ATR-FTIR data, the CN stretch 
disappeared, and the efficiency of the DSSCs decreased by 95\%. Even though the ATRFTIR peaks for the covalently-linked dye decreased by $\sim 20 \%$ for the same treatment, all of the peaks remained intact (including the $\mathrm{CN}$ stretch) and the efficiency of the DSSC with this PA actually increased by $\sim 35 \%$ as illustrated in Figure 3.13. We believe that the acid

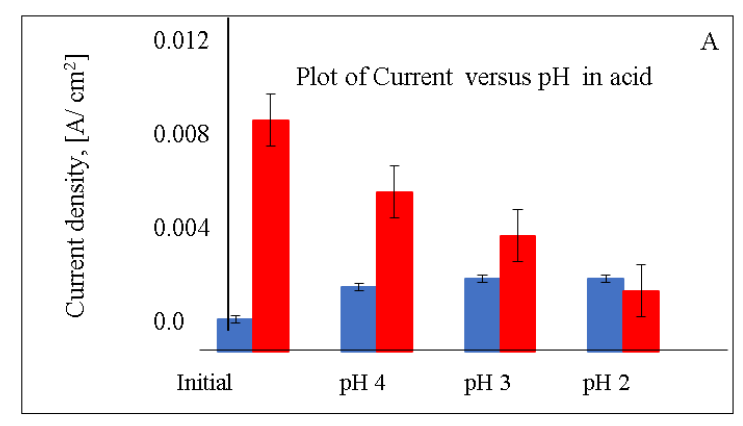

Photocurrent variation after treating with different strengths of an acid

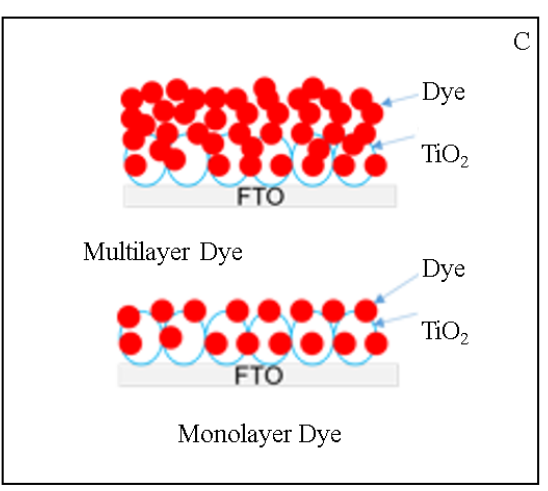

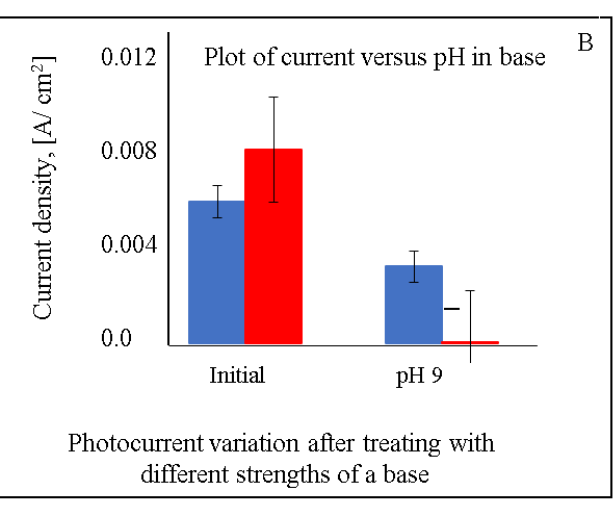

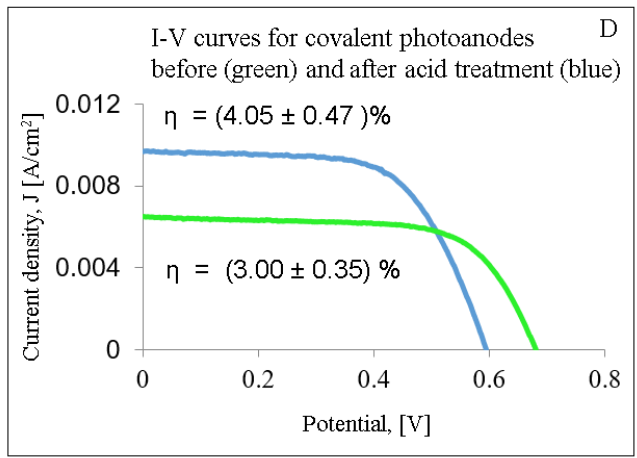

Increase of efficiency after treating with acid for $15 \mathrm{~min}, \mathrm{pH}$ at 2.5

Figure 3.13. (A) Photocurrent increased in covalent PAs after treating with acetic acid. (B)Both types of PAs, covalent and non-covalent did not show stability towards base. (C) Sketch of monolayer and multilayer on $\mathrm{TiO}_{2}$ after sensitization with $\mathrm{N} 719$ dye. In ACN:t-butanol solution, N719 is supposed to make a monolayer. (D) After multilayer was removed, covalent PAs showed an increase in efficiency from $3 \%$ to $4 \%$, a $33 \%$ increase.

treatment likely removed multilayers of N719 dye, while the dye attached covalently onto the $\mathrm{TiO}_{2} / \mathrm{APTES}$ remained stable. This led to a better dye monolayer on the $\mathrm{TiO}_{2}$ surface with enhanced photovoltaic performance. When excessive rinsing has been used to remove physisorbed multilayers on traditionally-attached dye, it also leads to removal of the chemisorbed dye, reducing efficiency. With covalently-attached dye, we can aggressively 
remove physisorbed multilayers without loss of the dye monolayer, increasing the efficiency.

Figure 3.14A shows ATR-FTIR spectra of traditionally prepared FTO/ $\mathrm{TiO}_{2} / \mathrm{Dye}$ (NC-1) and covalently-attached FTO/TiO2/APTES/Dye (C-3) PAs before and after soaking in water for $1 \mathrm{~h}$. For the PA with traditionally-attached dye, the carboxylate and bipyridyl
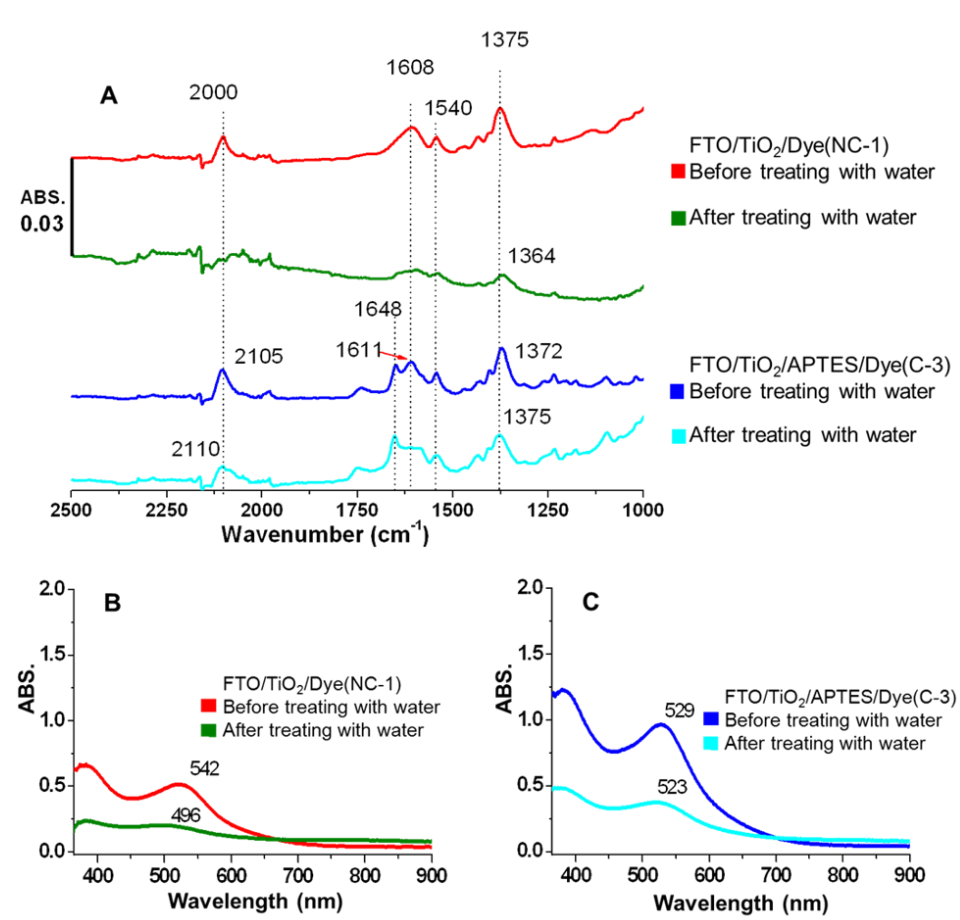

Figure 3.14. (A) ATR-FTIR spectra of conventional $\mathrm{FTO} / \mathrm{TiO}_{2} /$ Dye (NC-1) and covalent $\mathrm{FTO} / \mathrm{TiO}_{2} / \mathrm{APTES} / \mathrm{Dye}(\mathrm{C}-3)$ photoanodes before and after soaking in water for $1 \mathrm{~h}$. UV-vis spectra of (B) conventional $\mathrm{FTO} / \mathrm{TiO}_{2} /$ Dye (NC-3) photoanodes before and after soaking in water for $1 \mathrm{~h}$.

peaks decrease dramatically and the $\mathrm{CN}$ stretch completely disappears after soaking in water. The UV-vis spectra in Figure 3.14B also shows a dramatic absorbance decrease for the MLCT band of the dye and a large $46 \mathrm{~nm}$ blue shift of the peak, consistent with loss of the NCS ligands. For the PA with the covalently-linked dye, the carboxylate, bipyridyl, and NCS peaks decrease a little, but are still present and in a similar position. Interestingly, the amide peak at $1648 \mathrm{~cm}^{-1}$ does not decrease at all, showing high stability of the amide bond 
in the presence of water. The loss of the carboxylates, bipyridyl, and NCS bands may again be due to removal of multilayer N719 dye as discussed previously. The UV-vis spectra in Figure 3.14C shows a decrease in the MLCT band of the dye, but there is still a significant amount and the MLCT band only exhibited a blue shift of $6 \mathrm{~nm}$, consistent with a stable dye structure that retains the NCS ligands. Clearly, the ATR-FTIR and UV-vis show that the chemistry and optical properties of the dye are more stable for the covalently-bound dye in the presence of water.

\section{Discussions}

In this work, we describe a chemical approach for the surface modification of $\mathrm{TiO}_{2}$ mesoporous films with APTES followed by covalent attachment of dye through an amide bond. The covalent bonding between $\mathrm{TiO}_{2}$ and the dye through APTES decreased the efficiency compared to traditionally-prepared PAs, likely due to multilayer dye formation and poor electronic coupling between dye molecules and $\mathrm{TiO}_{2}$. Importantly, the PAs with covalently-linked N719 dye are air stable for more than 60 days and more resistant to UV light, thermal stress, acid, and water when compared to traditional PAs. The efficiency can also be improved by removing some of the multilayer by soaking in acid or water solution (Figure 3.13). In Figure 3.15 covalent PAs whose dye-coverage was made similar to optimized traditional PAs by monitoring through UV-Vis peaks, and I-V curves were recorded. Both types PAs gave nearly similar efficiency but $\mathrm{V}_{\mathrm{oc}}, \mathrm{FF}$ were clearly improved for covalent PAs. The soaking time for covalent PAs was reduced to $3 \mathrm{~h}$ from $24 \mathrm{~h}$ for traditional PAs. It is reported that $\mathrm{N} 719$ dye can make monolayer coverage after $24 \mathrm{~h}$ soaking in solution 1 (50:50 ratio of ACN:t-butanol). The UV-vis (Figure 3.15B) and ATR-FTIR (Figure 3.15C) gave almost the similar nature of peaks confirming that dye-coverage was 
same for both types of PAs. The ATR-FTIR spectroscopy experiments showed direct evidence for the formation of an amide bond between APTES and the dye. This amide I peak remained after the various treatments, showing that the covalent linkage was stable.

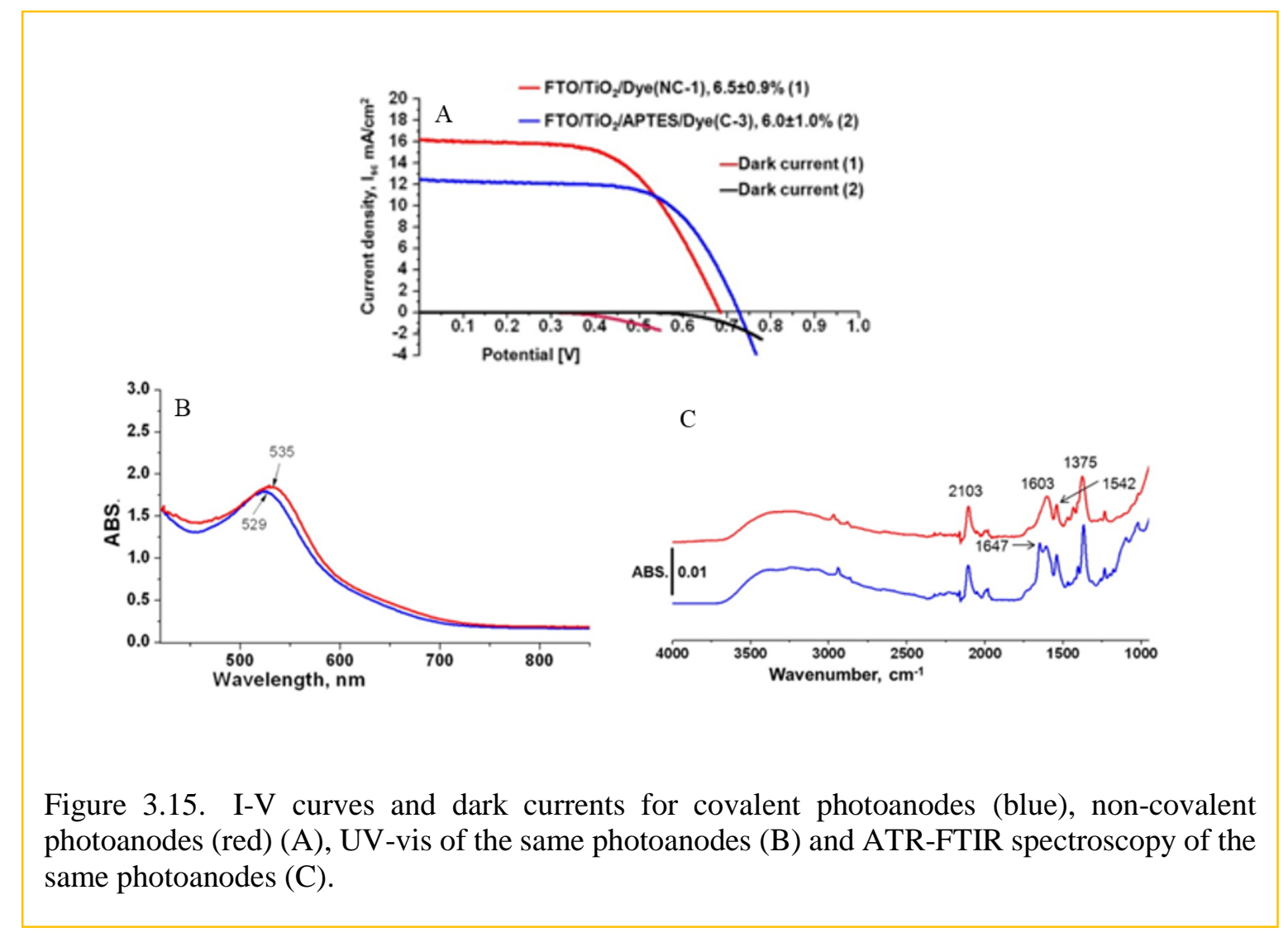

More importantly, the CN stretch from the NCS ligands was present for the covalentlylinked dye, but disappeared for the traditionally-prepared $\mathrm{FTO} / \mathrm{TiO}_{2} /$ Dye PAs after the various treatments. The loss of the $\mathrm{CN}$ stretch in the ATR-FTIR data correlated with a blue shift in the UV-vis spectra and loss in DSSC efficiency. This suggests that traditionallyprepared dye loses efficiency over time because of replacement of the NCS ligands of the dye with atmospheric water as described in a previous report. The covalent bonding of dye to APTES prevents, or dramatically slows down this replacement from occurring, leading to greater stability and no loss in efficiency for greater than 60 days in air. The various treatments accelerate the loss of the NCS band in the ATR-FTIR, leading to a blue shift in 
the UV-vis spectra and efficiency loss for traditionally-prepared PAs. While it is possible to seal traditionally-prepared PAs in a sandwich cell and obtain long term stability, the cells are not stable indefinitely. The PAs prepared with covalently-attached dye in this work are expected to show greatly improved stability in sealed sandwich cells when compared to those prepared traditionally, since degradation by UV, heat, acid, and water may still play a role. Our approach for attaching N719 dye covalently through APTES allows for air storable PAs, which provides added convenience and lower cost when considering the bulk-scale production of DSSCs. In the future, we will focus on obtaining a detailed understanding of the lower efficiency for the PAs with covalently-linked dye in order to develop a strategy to improve it, which would lead to the important goal of producing both highly efficient and highly stable DSSCs.

\subsection{Conclusions}

In this work we describe the chemical surface modification of mesoporous $\mathrm{TiO}_{2}$ films with APTES followed by covalent attachment of N719 dye through an amide bond for use as PAs in DSSCs. These covalently-linked PAs have similar efficiency as traditionallyprepared PAs (6-7\%), but are air stable for at least 60 days as compared to only a few days for traditionally-prepared PAs. They are also more resistant to UV light, thermal stress, acid, and water. ATR-FTIR spectra showed direct evidence for the formation of an amide bond between APTES and the dye. The amide I peak remained after the various treatments, showing a very stable covalent linkage. The $\mathrm{CN}$ stretch from the NCS ligands and $\mathrm{TBA}^{+}$ peaks in the ATR-FTIR were present for the covalently-linked dye, but disappeared quickly

for the traditionally-prepared PAs after the various treatments, suggesting that they play an 
important role in N719 dye stability. Those changes in IR correlated with a blue shift in the UV-vis spectra and loss in DSSC efficiency. The NCS ligands of the dye may be replaced with atmospheric water as described in a previous report. ${ }^{94}$ The covalent bonding of dye to APTES prevents, or dramatically slows down this replacement from occurring, leading to practically no loss in efficiency over several months. The various treatments accelerate the loss of the NCS band and TBA ${ }^{+}$cations in the ATR-FTIR, leading to a blue shift in the UVvis spectra and efficiency loss for traditionally-prepared PAs. Pre-modification of $\mathrm{TiO}_{2}$ is important to protect NCS ligand, the post modification of photoanode by octylamine shows increase in photovoltage maintaining the same current as compared to conventional, however, device performance for efficiency could not sustain more than 3 days (shown in Figure 3.16 A-C). In this case, $\mathrm{TiO}_{2}$ was first sensitized with $\mathrm{N} 719$ dye and then the free ends of carboxylate/carboxylic group was induced to make amide bond with octylamine in the same way as used to make amide bond. In fact, covalent modification could preserve every component of N719 when stored in air. For 6 month old sample, the data in Figure 3.17 shows that tetrabutylammonium ion, 2937 and $28860 \mathrm{~cm}^{-1}$ peaks, $\left(\mathrm{TBA}^{+}\right)$was in the surface of $\mathrm{TiO}_{2}$ even after 6 months stored in air, whereas it all went for conventional samples. When the ATR-FTIR spectra for every sample ware analyzed, $\mathrm{TBA}^{+}$was absent for conventional PAs even after 5 to 10 days. 


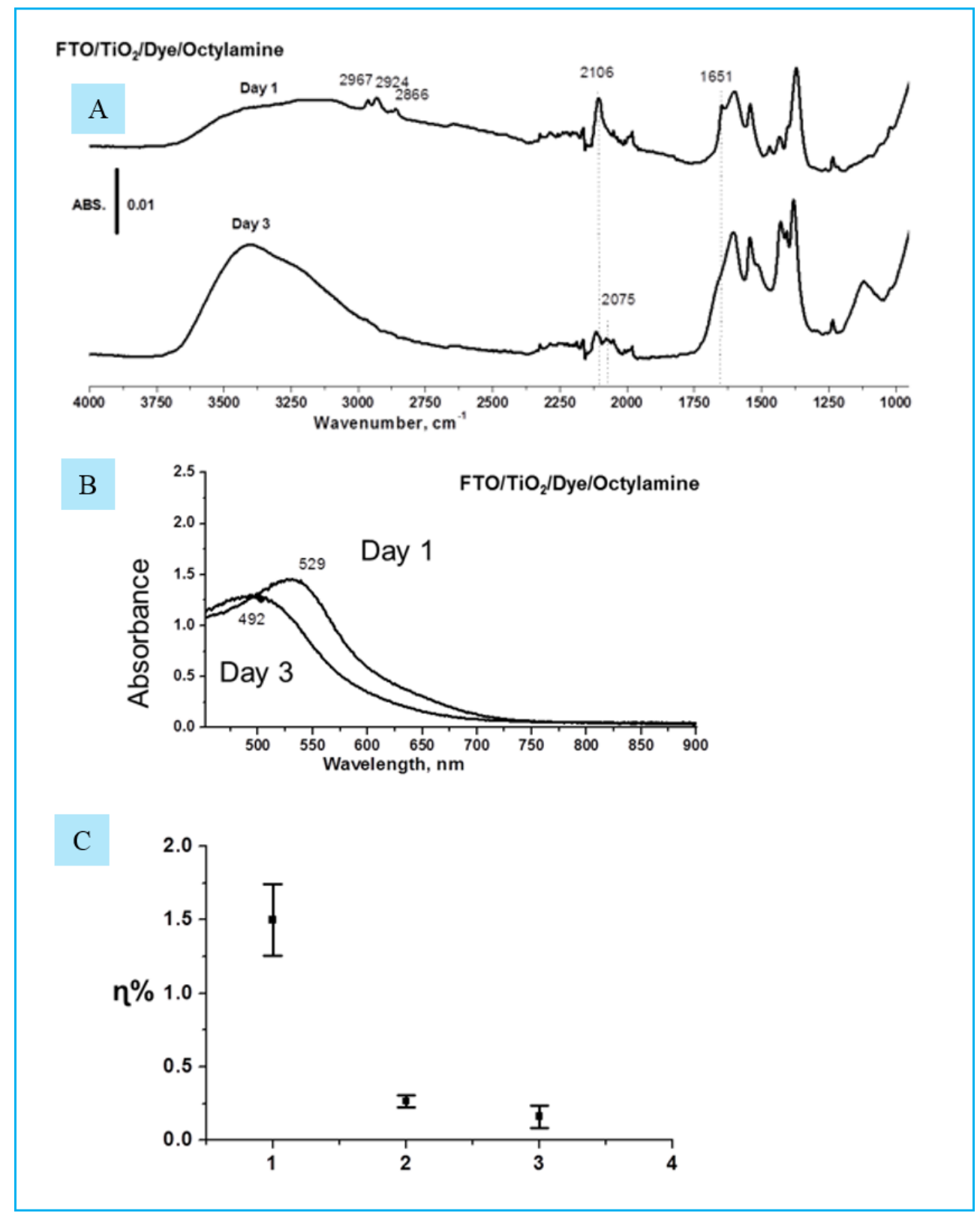

Figure 3.16. (A) ATR-FTIR data of $\mathrm{FTO} / \mathrm{TiO}_{2} /$ Dye functionalized with octylamine covalently through an amide bond on the first day of preparation "Day 1 " and the third day of exposure to air "Day 3". The dye is not stable based on the many changes in the IR spectrum. Figure (B). $\mathrm{UV}$-vis data of $\mathrm{FTO} / \mathrm{TiO}_{2} /$ Dye functionalized with octylamine covalently through an amide bond on the first day of preparation "Day 1" and the third day of exposure to air "Day 3". Figure (C). I-V curves of FTO/TiO $/$ Dye functionalized with octylamine covalently through an amide bond on the first day of preparation "Day 1" and the third day of exposure to air "Day 3". 


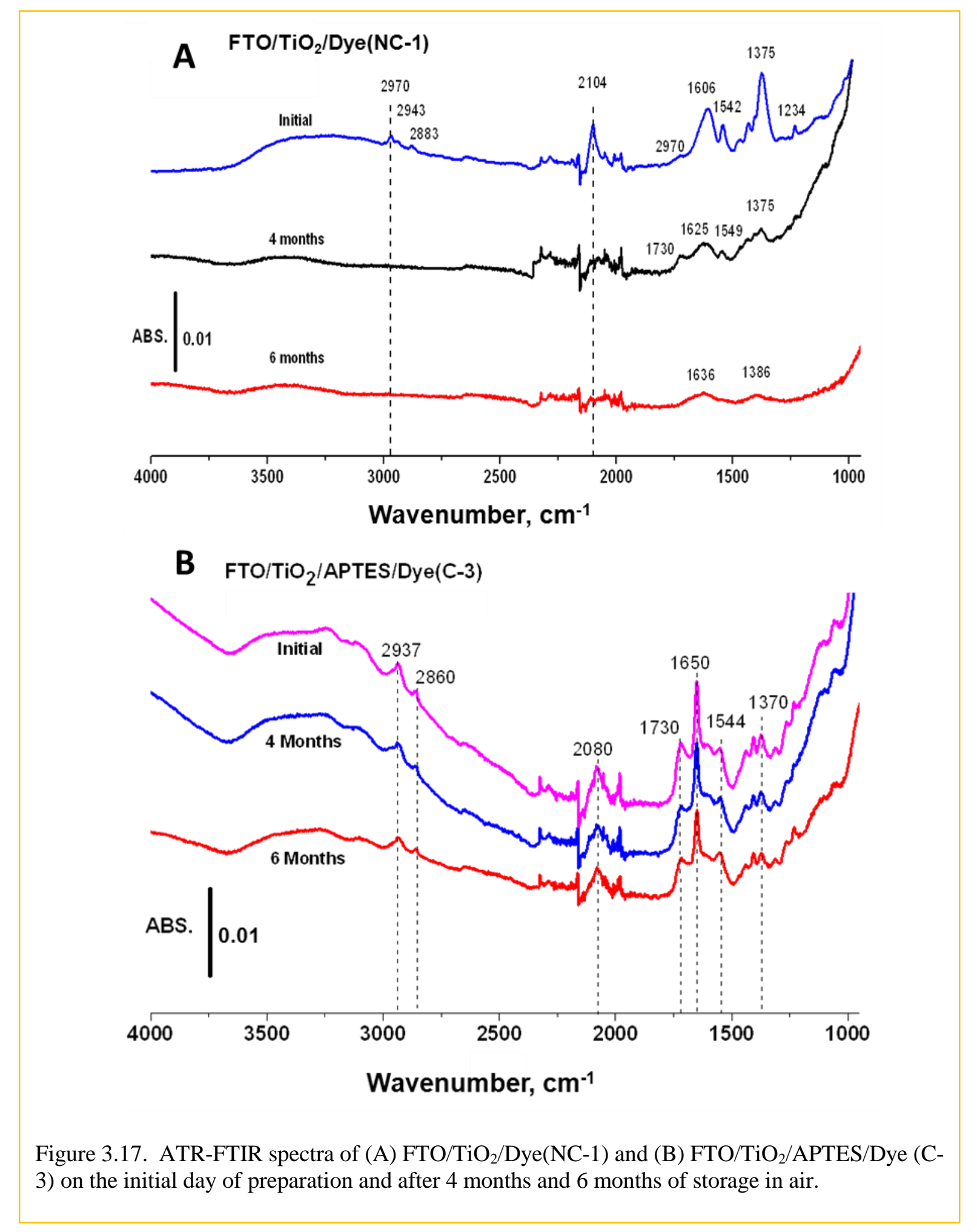




\section{CHAPTER 4}

\section{SPECTROSCOPIC INVESTIGATION OF PHOTOINDUCED CHARGE-TRANSFER PROCESSES IN FTO/TiO2/N719 PHOTOANODES WITH AND WITHOUT COVALENT ATTACHMENT THROUGH SILANE-BASED LINKERS}

Surface modification of $\mathrm{TiO}_{2}$ can bring significant changes on $\mathrm{TiO}_{2}$. In Chapter 3 we showed covalent photoanodes stable for up to 6 months. N719 dye was covalentlylinked to $\mathrm{TiO}_{2}$ through an amide bond, and as result, PAs did not degrade photovoltaic performance. As described in this chapter, charge injection dynamics from $\mathrm{N} 719$ to $\mathrm{TiO}_{2}$ was investigated by transient absorption pump-probe spectroscopy. Different types of molecular linkers were utilized and bound to $\mathrm{TiO}_{2}$ covalently and non-covalently. The spectroscopic results are interpreted on the basis of the standard electron transfer (ET) theory, and can be used to guide the selection of molecular linkers for DSSCs to produce better device performance. This research has been published in Journal of Physical Chemistry A. Based on the findings in this chapter, new devices were fabricated. It was revealed that new devices gave improved PCE values. Other factors that affect the efficiency and stability of the DSSCs are also discussed. 


\subsection{Introduction}

The dye-sensitized solar cell (DSSC) has become one of the most promising photovoltaic devices because of its low cost and relatively high power conversion efficiency (PCE). ${ }^{8,21,105-107}$ Fewer studies, however, have been carried out to optimize other factors of the device performance of DSSCs, especially their stability. Upon air exposure, a traditional DSSC photoanode loses its efficiency completely after 20 days. ${ }^{92,108}$ The efficiency is also affected by exposure to heat, water, acids, UV illumination, and desorption of the dye molecules from the semiconductor nanoparticles. ${ }^{94,109}$ Sealing the DSSCs improves their stability but cannot completely prevent long-term degradation. ${ }^{55}$

Performance of DSSCs can be improved by modification of photoanodes. Both premodification $^{99}$ and post-modification ${ }^{100}$ by 3 -aminopropyltriethoxysilane (APTES) have been studied previously to increase the efficiency of DSSCs using Ru-based dye. Recently, Luitel et al. used APTES as molecular linker to covalently attach N719 dye molecules to $\mathrm{TiO}_{2}$ films and observed a dramatic improvement in photoanode stability. The linker molecule is covalently attached to the N719 dye molecule via an amide bond and anchored to the $\mathrm{TiO}_{2}$ nanoparticle by its silane group. For DSSCs with the APTES-attached photoanodes, the PCE remains stable after air exposure for more than 60 days. However, the PCE of the DSSCs with covalently-attached photoanodes is reduced compared to DSSCs with conventional unmodified photoanodes. A 50\% drop in PCE (from $6 \%$ to $3 \%$ ) was observed for DSSCs with APTES-attached photoanodes when the soaking time in dye solution was the same. The PCE of solar cells strongly depends on photoinduced dynamics, which can be investigated by time-domain laser spectroscopy. Electron injection from the excited states of dye molecules to the conduction band (CB) of mesoporous semiconductor 
films usually occurs on a time scale of $<100$ fs to hundreds of picoseconds ${ }^{111-116}$ and consists of several competing processes. The back electron transfer occurs in the microsecond time range. ${ }^{117-121}$ To unravel the effect of covalent attachment using molecular linkers on the PCE of DSSCs, in the present work, the electron injection process in the covalently-attached photoanode with $\mathrm{FTO} / \mathrm{TiO}_{2} / \mathrm{APTES} / \mathrm{N} 719$ configuration was studied by ultrafast transient absorption pump-probe spectroscopy (TAPPS). To examine the effect of different linkers on the electron injection process an aromatic linker, p-aminophenyltrimethoxysilane $(\mathrm{APhS})$, was also investigated and compared to APTES. The conventional unmodified photoanode with the $\mathrm{FTO} / \mathrm{TiO}_{2} / \mathrm{N} 719$ configuration as well as the $\mathrm{N} 719$ solution was also studied for comparison. In addition, attenuated total reflectance-Fourier transform infrared (ATR-FTIR) and steady-state UV/visible absorption spectra of the photoanodes and the solution were obtained to help understand the time-resolved investigation. The experimentally observed spectra are used to explain the change in PCEs.

The comparative study of photoinduced electron-transfer (ET) processes in the presence of molecular linkers is also of significant fundamental interest. ET in bulk solid/liquid interfaces is generally described by the theoretical model first developed by Marcus, Gerischer, and Levich in the 1960s. ${ }^{121-123}$ The ET rate depends on both the relative energetics and the electronic coupling of the donor and the acceptor: the dye moleculesemiconductor nanoparticle junction can be regarded as a donor-bridge-acceptor (D-B-A) complex with a dye sensitizer (electron donor) attached to the semiconductor NP film surface (electron acceptor) through a molecular spacer and an anchoring group (bridge). The electronic coupling is determined by both the spacer and the anchoring group. Previously, the dependence of the electron injection rate on the bridge length has been studied using 
molecules with variable spacer units, e.g., methylene groups ${ }^{124}$ or p-phenylene groups ${ }^{98,125,126}$ In the present work, the electron donor and acceptor are connected by a linker molecule that is different from the dye sensitizer. It therefore provides a unique case to study the effect of bridges on ET dynamics.

\subsection{Experimental Details}

Preparation of $\mathrm{TiO}_{2}$ Film: FTO slides were cleaned by sonicating in acetone, EtOH, and IPA for 10 min each and dried under a $\mathrm{N}_{2}$ stream for $30 \mathrm{~s}$. The cleaned FTO slides were then soaked in a $40 \mathrm{mM}$ aqueous solution of $\mathrm{TiCl}_{4}$ and heated in a water bath at $70{ }^{\circ} \mathrm{C}$ for $30 \mathrm{~min}$. A $0.25 \mathrm{~cm}^{2}$ exposed area was made on the $\mathrm{TiCl}_{4}$ treated FTO slides by using Scotch tape. A small amount of DSL $(18 \mathrm{~nm}) \mathrm{TiO}_{2}$ paste was kept on one edge and spread by a sharp blade on the exposed part to obtain an active layer with uniform thickness. The deposited paste was sintered at $500{ }^{\circ} \mathrm{C}$ for $1 \mathrm{~h}$ in a 1300 BL Barnstead Thermolyne furnace. A second scattering layer (WREO-2, 150-250 nm) was deposited with the same method on the FTO slides and sintered at $500{ }^{\circ} \mathrm{C}$ for $1 \mathrm{~h}$. Finally, the film containing two layers of $\mathrm{TiO}_{2}$ pastes (active layer and scattering layers) was treated with $40 \mathrm{mM} \mathrm{TiCl}_{4}$ in water at $70{ }^{\circ} \mathrm{C}$ for $30 \mathrm{~min}$. This is referred to as the $\mathrm{FTO} / \mathrm{TiO}_{2}$ electrode. Note that for all TAPPS measurements, the scattering layer was not used.

Functionalization of $\mathrm{TiO}_{2}$ Film with APTES and APhS: Chemical Modification with APTES was performed according to Chapter 3. To study the effect of linker on charge transfer dynamics, aromatic linker (APhS) was used. The functionalization of $\mathrm{TiO}_{2}$ with $\mathrm{APhS}$ was done as follows. The $\mathrm{FTO} / \mathrm{TiO}_{2}$ electrode was placed inside a solution containing $20 \mathrm{~mL}$ of DMSO and $2 \mathrm{~g}$ of APhS and heated at $130{ }^{\circ} \mathrm{C}$ for $3 \mathrm{~h} .{ }^{128}$ The electrode was rinsed 
thoroughly with $\mathrm{EtOH}$ and dried under a $\mathrm{N}_{2}$ stream for $1 \mathrm{~min}$. This is referred to as the $\mathrm{FTO} / \mathrm{TiO}_{2} / \mathrm{APhS}$ electrode.

Previously, it was reported that, when $\mathrm{SiO}_{2}$ was used as substrate, the primary-amine coverage using 3-aminopropyltrimethoxysilane (APTMS) and APhS could reach as high as $88.6 \%$ and $100 \%$, respectively. ${ }^{129}$ Similar coverage of APTES and $\mathrm{APhS}$ on $\mathrm{TiO}_{2}$ is expected.

Dye Sensitization of $\mathrm{TiO}_{2}$ Film and Covalent Attachment: Sensitization of the $\mathrm{FTO} / \mathrm{TiO}_{2}$ electrodes was achieved by immersing them into a $0.3 \mathrm{mM}$ solution of $\mathrm{N} 719$ dye in a 1:1 mixture of acetonitrile and tert-butanol. ${ }^{130}$ The electrodes were soaked in a dye solution for $24 \mathrm{~h}$. To prepare photoanodes with covalently-attached dye sensitizer, the functionalized $\mathrm{FTO} / \mathrm{TiO}_{2} / \mathrm{APTES}$ and $\mathrm{FTO} / \mathrm{TiO}_{2} / \mathrm{APhS}$ electrodes were soaked in $0.3 \mathrm{mM}$ dye solution containing $20 \mathrm{mM}$ of DMAP and $20 \mathrm{mM}$ of DCC in $10 \mathrm{~mL}$ of methylene chloride. ${ }^{98}$ This makes the covalent attachment of dye molecules with APTES and APhS linkers via amide bonds. They are referred to as $\mathrm{FTO} / \mathrm{TiO}_{2} / \mathrm{APTES} / \mathrm{N} 719$ and $\mathrm{FTO} / \mathrm{TiO}_{2} / \mathrm{APhS} / \mathrm{N} 719$ photoanodes. Interactions between dye and $\mathrm{FTO} / \mathrm{TiO}_{2}$, FTO/TiO $2 / \mathrm{APTES}$, and $\mathrm{FTO} / \mathrm{TiO}_{2} / \mathrm{APhS}$ are depicted in Figure 4.1 a-c. Given their four carboxyl/carboxylic acid groups, $\mathrm{N} 719$ dye molecules may attach to the $\mathrm{TiO}_{2}$ nanoparticles via multiple linker molecules. Parts a-c of Figure 4.1 show only the single attachment situation. An accurate measurement of the percentages of the singly and multi-attached dye molecules was not attempted.

Spectroscopic Characterization: To determine how APTES and APhS interact with the N719 dye, spectroscopic studies were carried out to characterize the photoanodes, including attenuated total reflectance-Fourier transform infrared (ATR-FTIR) and 
UV/visible absorption spectroscopy. In ATR-FTIR measurement, photoanodes were placed onto the ZnSe crystal in the spectrometer (Perkin-Elmer, Spectrum 100) with the dye layer facing the crystal and held at a pressure of $80 \mathrm{psi}$. Scans were taken from 400 to $4000 \mathrm{~cm}^{-}$

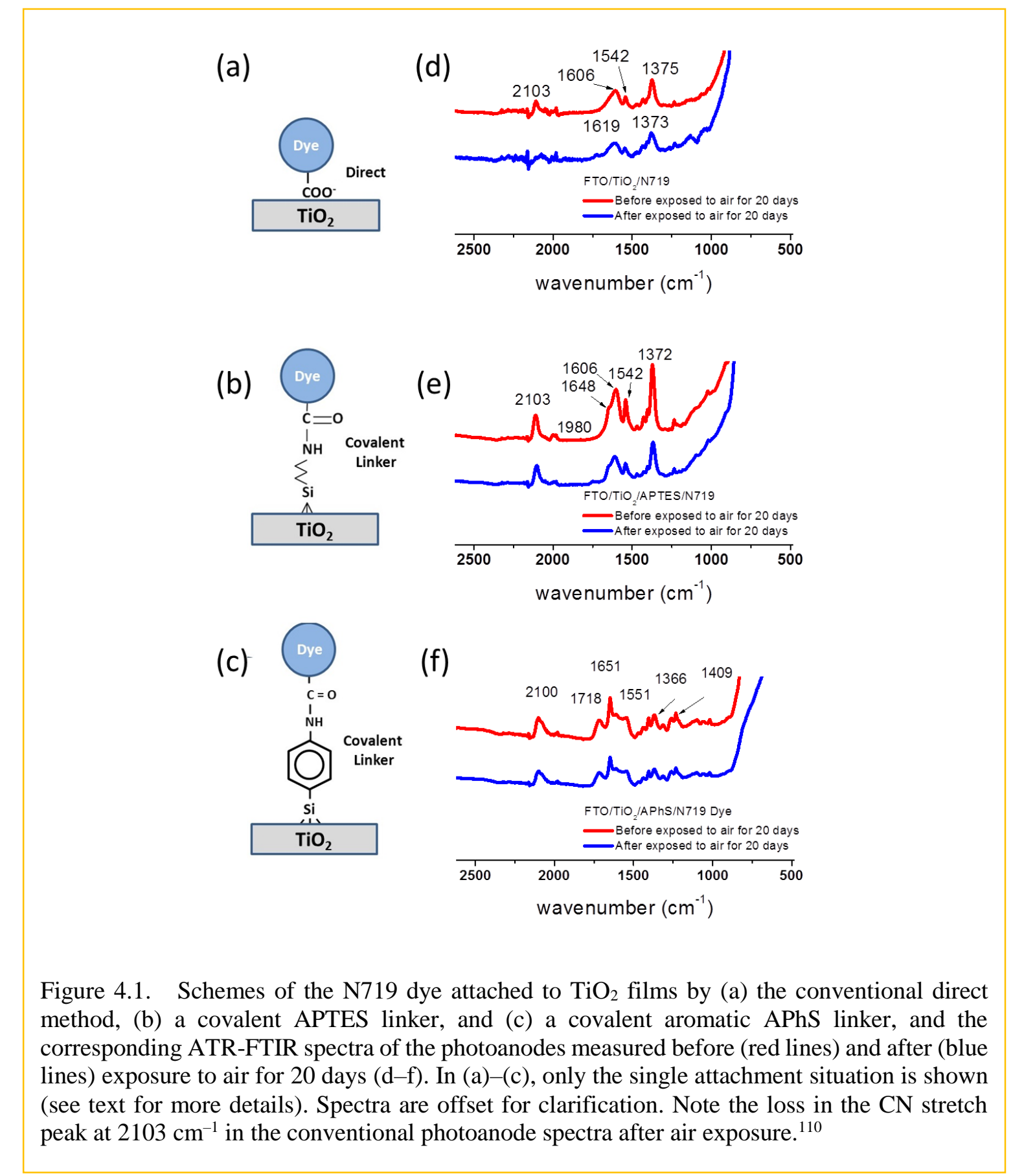

${ }^{1}$ with 20 averages and a resolution of $4 \mathrm{~cm}^{-1}$. To reveal the mechanism for stabilization of 
photoanodes by covalent linkers, spectra before and after air exposure for 20 days were obtained.

UV/visible absorption spectra of the covalently-attached photoanodes were obtained with a Varian Cary Win 50 UV-vis spectrophotometer. Absorption spectra of N719 in (1:1) $\mathrm{ACN}$ and tert-butanol solution and conventional $\mathrm{FTO} / \mathrm{TiO}_{2} / \mathrm{N} 719$ photoanodes were also recorded for comparison.

Ultrafast Transient Absorption Pump-Probe Spectroscopy (TAPPS): Ultrafast transient absorption pump-probe spectroscopy (TAPPS) measurements were performed on N719 in (1:1) ACN and tert-butanol solution on conventional FTO/TiO $/ 2$ N719 photoanode and on covalently-attached $\mathrm{FTO}_{\mathrm{TiO}} / \mathrm{APTES} / \mathrm{N} 719$ and FTO/TiO$/ \mathrm{TP}_{2} / \mathrm{PhS} / \mathrm{N} 719$ photoanodes. The TAPPS system uses a Clark-MXR CPA Ti:sapphire ultrafast laser (wavelength $=775 \mathrm{~nm}$, pulse duration $\lesssim 150 \mathrm{fs}$, pulse energy $\sim 1 \mathrm{~mJ}$ at $1 \mathrm{kHz}$ repetition rate). Output from the CPA is split and used to pump two noncollinear optical parametric amplifiers (NOPAs), which in total are able to cover a wavelength region 450-1600 nm. Part of the CPA output is also separated to pump a $3 \mathrm{~mm}$ thick sapphire plate to generate supercontinuum white-light $(420-1600 \mathrm{~nm})$. Output from the first NOPA at $530 \mathrm{~nm}$ wavelength was pulse-compressed to $\sim 30 \pm 5$ fs determined by an autocorrelation measurement. It was used as the pump light source. The pulse energy of the pump light is attenuated to below $0.2 \mu \mathrm{J}$ to avoid unwanted multiphoton excitation and thermal degradation of the samples. Both the white-light and the $860 \mathrm{~nm}$ output from the second NOPA were used as the probe light. In all measurements reported here, polarizations of the pump and probe beams are parallel. The probe beam is focused onto the sample while the pump beam is slightly defocused to ensure the coverage of the probe beam. After the sample, 
the pump beam is blocked by a notch filter. When the $860 \mathrm{~nm}$ probe beam was used, its transmission signal was detected with a Si photodiode (Thorlabs DET10A) and amplified by a lock-in amplifier (SRS SR810), whereas with the white-light probe beam, the transmission is dispersed by a curved grating and detected by a linear array CMOS detector in the wavelength range $430-730 \mathrm{~nm}$. The time delay between pump and probe pulses $(\Delta t)$ is variable between 0 and $1.5 \mathrm{~ns}$ by moving a retroreflector on a computer-controlled translation stage that reflects the pump beam. An optical chopper revolving at $500 \mathrm{~Hz}$, half the repetition rate of the femtosecond laser, is used to modulate the pump beam and the TAPP signal is recorded as the change in optical density $(\Delta \mathrm{OD})$ with pump beam blocked

$$
\Delta \mathrm{OD}(\Delta t)=-\log \left[\frac{I^{*}(\Delta t)}{I_{0}}\right]
$$

and unblocked: where $I_{0}$ and $I^{*}$ are the probe beam transmission with the preceding pump beam blocked and unblocked, respectively. Time zero was determined by the transient absorption signal of R6G dye solution, and the group velocity dispersion was corrected. The TAPPS system is controlled by a LabVIEW (National Instruments) program.

\subsection{Results and Discussions}

ATR-FTIR Spectra: ATR-FTIR spectra of the photoanodes with and without covalent attachment before and after air exposure are compared in Figure 4.1d-f. The most remarkable change in the spectrum of the conventional photoanode (Figure 4.1d) is the $\mathrm{CN}$ stretch from the two SCN ligands of the N719 dye molecule at $2103 \mathrm{~cm}^{-1}$. It almost completely disappeared after 20 days of air exposure. On the contrary, although decreased, it remained strong in the spectra of photoanodes such as $\mathrm{FTO}^{\mathrm{TiO}}{ }_{2} / \mathrm{APTES} / \mathrm{N} 719$ (Figure 1e) and $\mathrm{FTO} / \mathrm{TiO}_{2} / \mathrm{APhS} / \mathrm{N} 719$ (Figure 4.1f). Such observation suggests that the improvement in stability of the covalently-attached photoanodes is because the linker 
molecules provide an environment that protects the SCN ligands and retains the photoelectrochemical properties of the dye. Detailed analysis of the spectra and assignment of other IR peaks can be found elsewhere. ${ }^{108,110}$

Steady-State UV/Visible Absorption Spectra: Steady-state absorption spectra of N719 dye in solution and adsorbed on $\mathrm{TiO}_{2}$ film with and without covalent linkers (APTES and $\mathrm{APhS}$ ) are shown in Figure 4.2. In the spectrum of N719 in (1:1) ACN and tert-butanol solution (Figure 4.2a), two absorption maxima are observed at 380 and $536 \mathrm{~nm}$, which are assigned as metal-to-ligand charge-transfer (MLCT) transitions $\left(4 \mathrm{~d}-\pi^{*}\right) .{ }^{131}$ The long wavelength tail (up to $750 \mathrm{~nm}$ ) was attributed to transition to the luminescent $[\mathrm{RuIII}(\mathrm{bpy}) 2 \mathrm{bpy}-]^{2+}$ excited state. ${ }^{132}$ In the spectrum of the conventional photoanode, in which the N719 dye is directly adsorbed on $\mathrm{TiO}_{2}$ NPs (Figure 4.2b), the two absorption bands at 380 and $536 \mathrm{~nm}$ are red-shifted by 12 and $8 \mathrm{~nm}$, respectively, as compared to absorptions of the N719 dye solution. Previously the red shift was attributed to either the aggregation of dye molecules on the $\mathrm{TiO}_{2}$ surface ${ }^{133,134}$ or protonation equilibria caused by $\mathrm{Ti}_{4}{ }^{+}$as the dye interacts with $\mathrm{TiO}_{2}{ }^{62}$ The red shift effect is altered by the linker molecules. Parts $\mathrm{c}$ and d of Figure 4.2 show the absorption spectra of N719 dye covalently attached to $\mathrm{TiO}_{2}$ NPs by APTES and APhS, respectively. The insertion of APTES between $\mathrm{TiO}_{2}$ and the N719 dye causes a blue shift of the absorption peaks compared to the case of the conventional $\mathrm{FTO} / \mathrm{TiO}_{2} / \mathrm{N} 719$ photoanode. A blue shift with essentially the same magnitude is observed in the $\mathrm{UV} /$ visible spectrum of the $\mathrm{FTO} / \mathrm{TiO}_{2} / \mathrm{APhS} / \mathrm{N} 719$ photoanode. The observed blue shifts in $\mathrm{FTO} / \mathrm{TiO}_{2} / \mathrm{APTES} / \mathrm{N} 719$ and $\mathrm{FTO} / \mathrm{TiO}_{2} / \mathrm{APhS} / \mathrm{N} 719$ photoanodes suggest that the red shift in the absorption spectrum of the N719 dye upon adsorption on $\mathrm{TiO}_{2}$ NPs is mainly due to the interaction between the dye sensitizer and the semiconductor 
NPs, which can be altered by different choice of the molecular linker, and hence supports the aforementioned protonation-equilibria interpretation. Furthermore, a significant increase in absorption was observed for the covalently-attached photoanodes compared to the

(a)

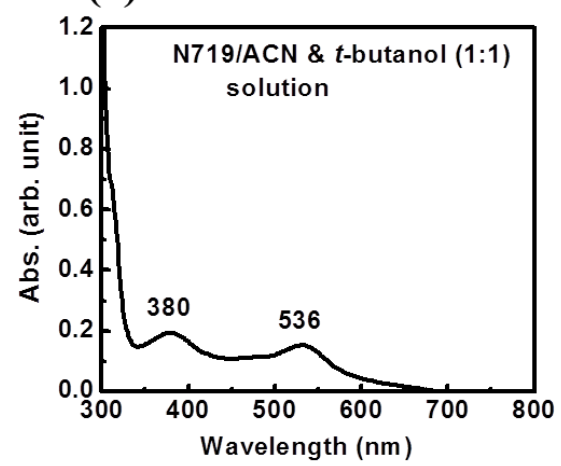

(c)

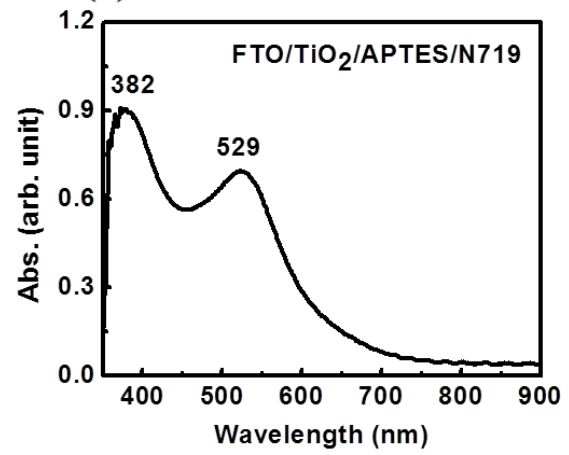

(b)

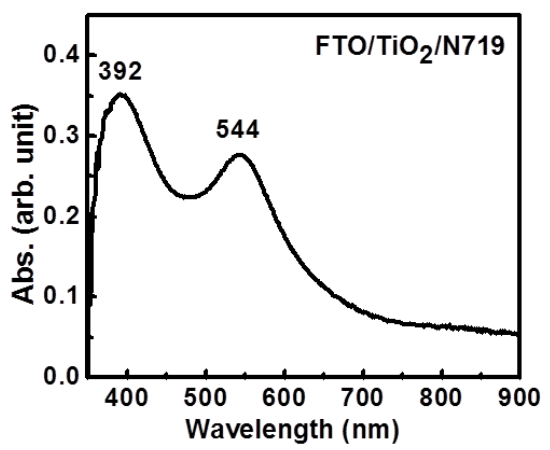

(d)

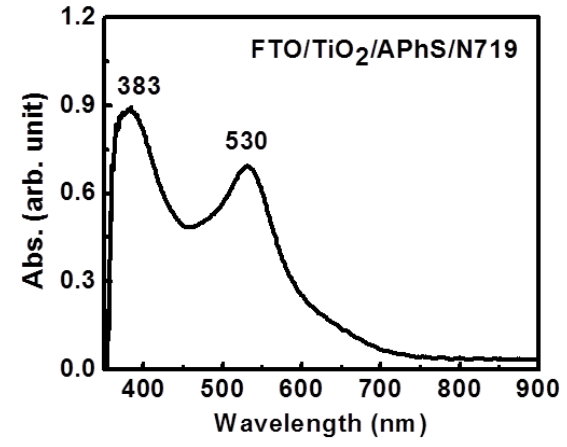

Figure 4.2. Steady-state UV/visible absorption spectra of (a) N719 dye in ACN:t-butanol (1:1) solution and (b) $\mathrm{FTO} / \mathrm{TiO}_{2} / \mathrm{N} 719$, (c) $\mathrm{FTO} / \mathrm{TiO}_{2} / \mathrm{APTES} / \mathrm{N} 719$, and (d) $\mathrm{FTO} / \mathrm{TiO}_{2} / \mathrm{APhS} / \mathrm{N} 719$ photoanodes.

conventional one, suggesting multilayer and aggregation of dye molecules.

TAPP Spectra: The TAPPS experiment directly measures the electron injection dynamics in the photoanodes by varying the time delay between the pump and probe pulses. It also reveals details of the electron injection process by the probe-wavelength dependence. In the present work, $530 \mathrm{~nm}$ pump source was used to excite N719 to its MLCT band (Figure 4.2). Two probe light sources were used: single-wavelength $860 \mathrm{~nm}$ output from a NOPA 
and super-continuum white light. Due to its relative simplicity, the result with the $860 \mathrm{~nm}$ probe wavelength is presented first.

TAPP Spectra with 860 nm Probe Beam: Figure 4.3 shows transient absorption kinetics of $\mathrm{FTO} / \mathrm{TiO}_{2} / \mathrm{N} 719, \quad \mathrm{FTO}_{\mathrm{TiO}} / \mathrm{APTES} / \mathrm{N} 719$, and $\mathrm{FTO} / \mathrm{TiO}_{2} / \mathrm{APhS} / \mathrm{N} 719$

(a)

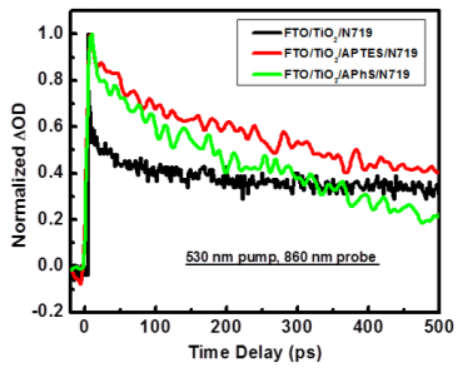

(c)

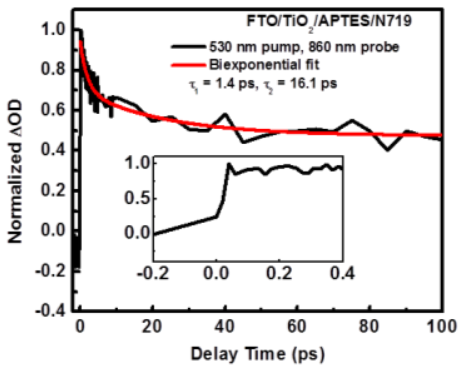

(b)

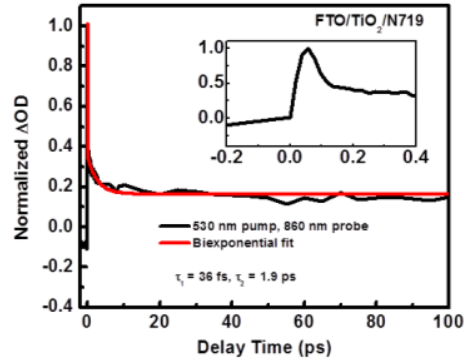

(d)

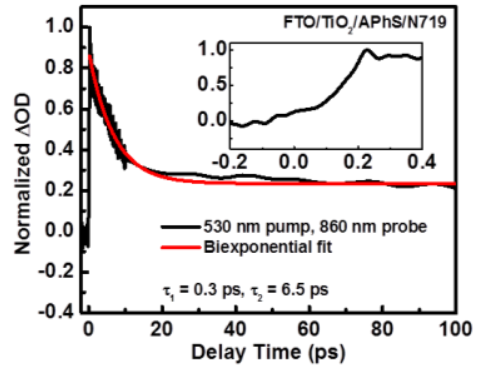

Figure 4.3. (a) Normalized transient absorption kinetics observed with the $530 \mathrm{~nm}$ pump wavelength and $860 \mathrm{~nm}$ probe wavelength of $\mathrm{FTO} / \mathrm{TiO}_{2} / \mathrm{N} 719, \mathrm{FTO} / \mathrm{TiO}_{2} / \mathrm{APTES} / \mathrm{N} 719$, and FTO/TiO $2 / \mathrm{APhS} / \mathrm{N} 719$ photoanodes. (b)-(d) illustrate the kinetics up to $100 \mathrm{ps}$ and the biexponential fits.

photoanodes up to $500 \mathrm{ps}$ with an excitation wavelength of $530 \mathrm{~nm}$ and a probe wavelength of $860 \mathrm{~nm}$. The decay curves are biphasic and can be fit to a biexponential function as follows: with $\tau_{1}<\mathrm{A}_{02}$. The fit parameters for the three photoanodes are listed in Table 4.1. It has been generally accepted that the faster and slower decay components correspond to electron injection from the singlet and triplet MLCT states, respectively. ${ }^{135-138}$ Previously, decay dynamics in shorter than $10 \mathrm{fs}$ were observed by ultrafast time-gate fluorescence spectroscopy ${ }^{138}$ and attributed to a nonergodic electron injection process from 
nonequilibrated singlet excited states of $\mathrm{N} 719$ to $\mathrm{TiO}_{2}$. Limited by the time resolution of the probe source (60 fs), such a fast process was not observed in the present experiment.

The fit curves as well as experimentally measured kinetics are illustrated and

\begin{tabular}{|c|c|c|c|c|c|c|}
\hline \multicolumn{7}{|c|}{ Table 4.1. Fit parameters for TAAPS kinetics } \\
\hline $\begin{array}{c}\text { Probe } \\
\text { wavelength } \\
\text { (nm) }\end{array}$ & Photoanodes & $\begin{array}{c}A_{0} \\
\text { (A.U.) }\end{array}$ & $\begin{array}{c}A_{1} \\
\text { (A.U.) }\end{array}$ & $\begin{array}{c}\tau_{1} \\
\text { (ps) }\end{array}$ & $\begin{array}{c}A_{2} \\
\text { (A.U.) }\end{array}$ & $\begin{array}{c}\tau_{2} \\
\text { (ps) }\end{array}$ \\
\hline \multirow{3}{*}{860} & $\mathrm{FTO} / \mathrm{TiO}_{2} / \mathrm{N} 719$ & $0.163 \pm 0.004$ & $0.661 \pm 0.015$ & $0.036 \pm 0.002$ & $0.191 \pm 0.005$ & $1.928 \pm 0.111$ \\
\hline & $\mathrm{FTO} / \mathrm{TiO}_{2} / \mathrm{APTES} / \mathrm{N} 719$ & $0.473 \pm 0.019$ & $0.242 \pm 0.026$ & $1.364 \pm 0.230$ & $0.228 \pm 0.023$ & $16.071 \pm 6.136$ \\
\hline & $\mathrm{FTO} / \mathrm{TiO}_{2} / \mathrm{APhS} / \mathrm{N} 719$ & $0.233 \pm 0.037$ & $0.322 \pm 0.027$ & $0.304 \pm 0.034$ & $3.988 \pm 3.561$ & $6.489 \pm 1.962$ \\
\hline \multirow{3}{*}{690} & $\mathrm{FTO} / \mathrm{TiO}_{2} / \mathrm{N} 719$ & $0.263 \pm 0.009$ & $0.765 \pm 0.007$ & $2.317 \pm 0.602$ & & \\
\hline & $\mathrm{FTO} / \mathrm{TiO}_{2} / \mathrm{APTES} / \mathrm{N} 719$ & $0.607 \pm 0.015$ & $0.338 \pm 0.013$ & $4.423 \pm 0.316$ & & \\
\hline & $\mathrm{FTO} / \mathrm{TiO}_{2} / \mathrm{APhS} / \mathrm{N} 719$ & $0.335 \pm 0.054$ & $0.642 \pm 0.041$ & $3.267 \pm 0.423$ & & \\
\hline
\end{tabular}

compared in Figure 4.3 (for $860 \mathrm{~nm}$ probe wavelength) and Figure 4.7 (for $690 \mathrm{~nm}$ probe wavelength). The time scales $\left(\tau_{1}\right.$ and $\left.\tau_{2}\right)$ are significantly different among the three photoanodes. Upon insertion of APTES linker, the time scale for the electron injection process from the singlet states of $\mathrm{N} 719$ to the conduction band of $\mathrm{TiO}_{2}\left(\tau_{1}\right)$ increases from 36 fs to $1.4 \mathrm{ps}$, whereas that from the triplet states $\left(\tau_{2}\right)$ increases from 1.9 to $16.1 \mathrm{ps}$. The increment in decay time scale is less significant when the $\mathrm{APhS}$ linker is used: to $\tau_{1}=0.3 \mathrm{ps}$ and $\tau_{2}=7.4 \mathrm{ps}$. Note that the fit values of the time scales of the fast decay component $\left(\tau_{1}\right)$, 
especially for the conventional photoanode, are comparable to the pulse duration and the instrument response function (IRF) of the TAPPS system. The fit values of $\tau_{1}$ are therefore not accurate measures of the electron injection rates but should be regarded as only semiquantitative estimates. Comparison among the three photoanodes, however, demonstrates the effect of the molecular linkers in hindering the electron injection processes.

A rise time of $50 \mathrm{fs}$ prior to the decay has been observed in TAPP spectra of the FTO/TiO $2 /$ N719 photoanode (inset of Figure 4.3b). Such a rise time is dominated by IRF but also contains contributions from intermolecular vibrational redistribution (IVR) and internal conversion (IC) in both the singlet and triplet excited-state N719 molecules, which equilibrate the molecules. A similar rise time was observed for the $\mathrm{FTO} / \mathrm{TiO}_{2} / \mathrm{APTES} / \mathrm{N} 719$ photoanode (inset of Figure 4.3c), whereas it is prolonged when APhS is used as the linker (inset of Figure 4.3d) probably due to the effect of the aromatic ring on the IVR and IC dynamics. Quantitative analysis, however, was not attempted due to the limited time resolution and instrumental response. It is worth noting that an offset, $\mathrm{A}_{0}$ in eq 2, was introduced in the biexponential fitting of all three TAPP decay dynamics. Such a longlasting signal can only be attributed to triplet state dye molecules that are not subject to ET processes due to poor coupling. Previously, a slow component of the observed electron injection process in $\mathrm{TiO}_{2} / \mathrm{N} 719$ solar cell was attributed to dye molecules that are loosely attached onto the surface or are present in an aggregated form. ${ }^{139}$ Such an offset is therefore evidence of multilayer or aggregates of N719 dye molecules. Because only the first layer of molecular sensitizer undergoes efficient electron injection to $\mathrm{TiO}_{2} \mathrm{NPs}, \mathrm{N} 719$ molecules in other layers can stay in triplet states after photoexcitation and the ensuing ISC. The $\mathrm{A}_{0}$ value is larger for $\mathrm{FTO} / \mathrm{TiO}_{2} / \mathrm{APTES} / \mathrm{N} 719$ and $\mathrm{FTO} / \mathrm{TiO}_{2} / \mathrm{APhS} / \mathrm{N} 719$ photoanodes than the 
conventional one, which partially explains their relatively lower PCE. It is also consistent with the stronger UV/visible absorption observed for these two photoanodes. (Compare Figure 4.2c,d to Figure 4.2b.)

TAPP Spectra with White-Light Probe: TAPP spectra using white-light as the probe source and detected in the $430-730 \mathrm{~nm}$ region are illustrated in Figure 4.4. The step size of the delay time was relatively large (3 ps) in obtaining the spectra in Figure 4.4. The

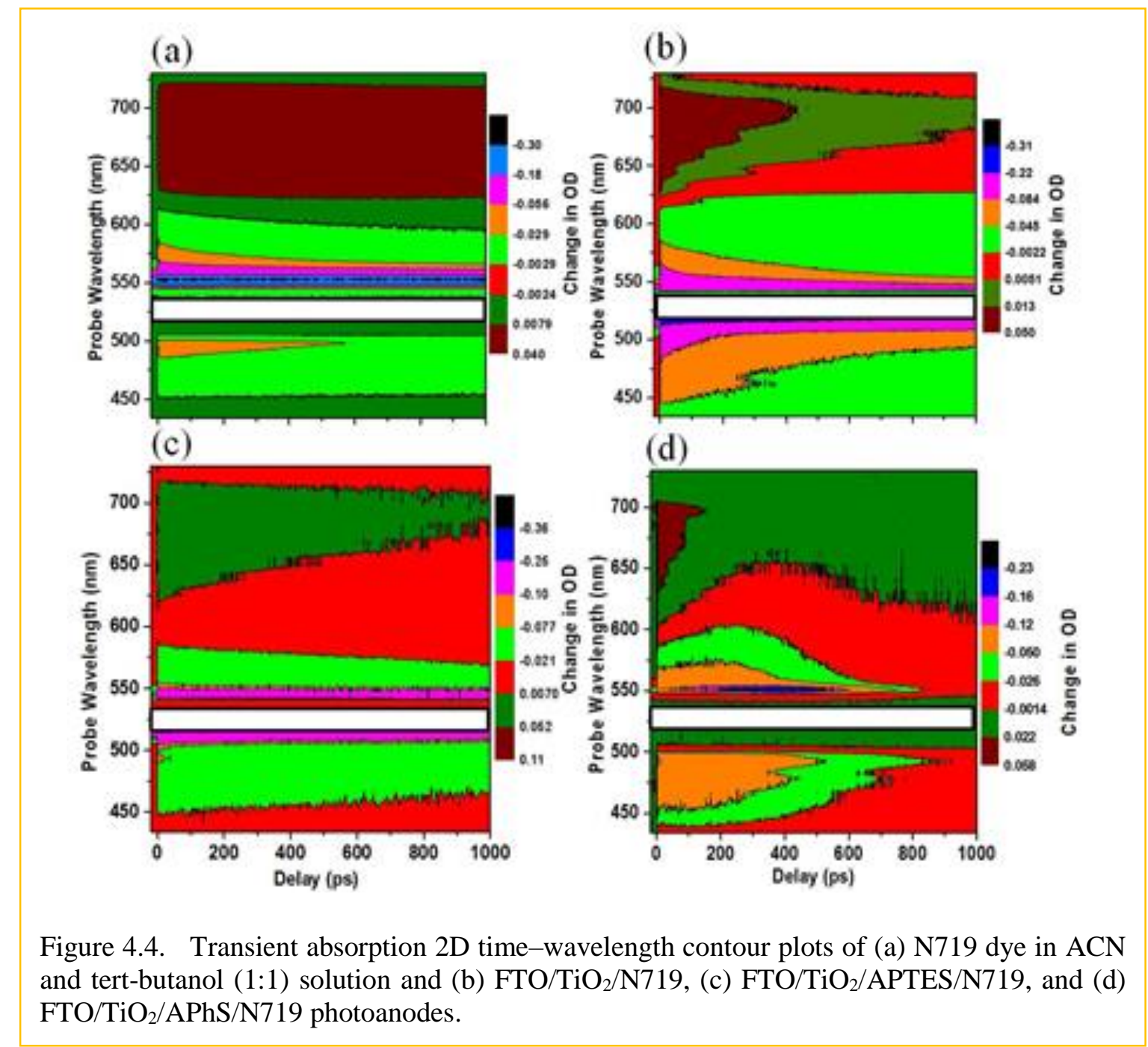

time--wavelength plots of all four samples, N719 dye dissolved in 1:1 ACN and tert-butanol solvent and $\mathrm{FTO} / \mathrm{TiO}_{2} / \mathrm{N} 719, \quad \mathrm{FTO} / \mathrm{TiO}_{2} / \mathrm{APTES} / \mathrm{N} 719$, and $\mathrm{FTO} / \mathrm{TiO}_{2} / \mathrm{APhS} / \mathrm{N} 719$ photoanodes, show a similar pattern: signals in the wavelength region longer than $600 \mathrm{~nm}$ 
are predominantly positive, whereas those in the shorter wavelength region are predominantly negative. The positive signals are due to excited-state absorption (ESA) and directly related to the formation and relaxation of excited (singlet or triplet) states and oxidized states of the dye molecules after photoexcitation. The negative signals are attributed to the ground-state bleach (GSB). We rule out any major contribution from stimulated emission (SE) to the negative signals on the basis of two facts: (1) the negative signal is roughly symmetric on both sides of the pump wavelength $(530 \mathrm{~nm})$. Due to the fast IVR/IC ( $<10 \mathrm{fs})$ and ISC ( $<30 \mathrm{fs})$ processes the SE process would contribute mostly in the longer wavelength region and make the spectra asymmetric. (2) No evidence of SE was found in the positive-signal region with wavelength >600 nm except for the N719 solution because the decay kinetics are single-exponential within 10 ps delay time (see below).

From the $2 \mathrm{D}$ contour plots, transient absorption spectra in the 430-730 nm range at different delay times $(\Delta \mathrm{t}=0.5 \mathrm{ps}, 10 \mathrm{ps}, 100 \mathrm{ps}, 500 \mathrm{ps}$, and $1 \mathrm{~ns})$ are extracted and shown in Figure 4.5. Time evolution of the transient absorption spectrum of the N719 dye solution (Figure 4.5a) is different in the two wavelength regions longer and shorter than $600 \mathrm{~nm}$. The ESA signal in the $>600 \mathrm{~nm}$ region builds up completely within 100 ps after photoexcitation and thereafter decays slowly on a time scale much longer than nanoseconds. In contrast, the GSB signal in the $<600 \mathrm{~nm}$ region demonstrates a continuous decrease starting from pump-probe delay time of $0.5 \mathrm{ps}$ due to the ground-state recovery. In the transient absorption spectra of the three photoanodes (Figures $\mathbf{5 b} \mathbf{b}-\mathbf{d}$ ), similar ground-state recovery dynamics were observed. However, the dynamics of the ESA signals are different from those of the solution. No buildup was observed; instead, one sees the decay of ESA signal immediately after photoexcitation. On the basis of these observations, we conclude 
that the buildup behavior observed for the positive signal of the N719 solution in the region longer than $600 \mathrm{~nm}$ is actually a combined effect of ESA and SE: an SE process with a time

(a)

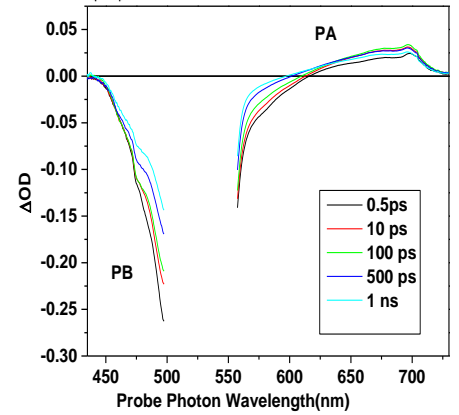

(c)

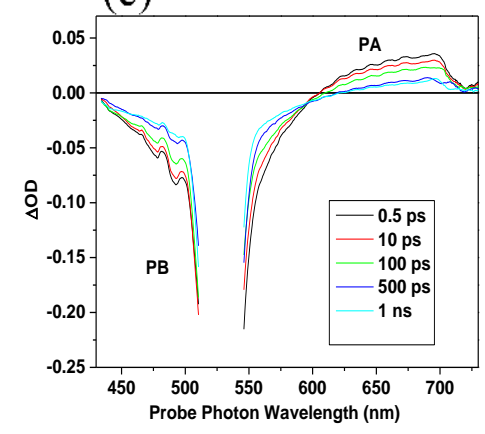

(b)

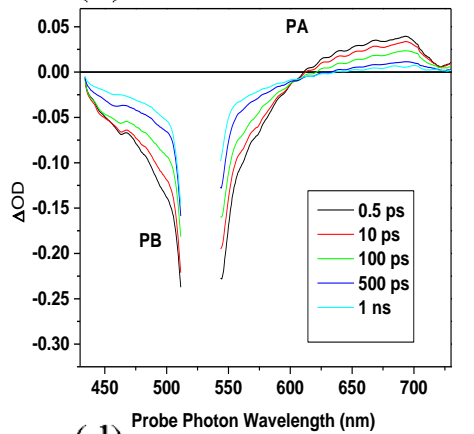

(d)

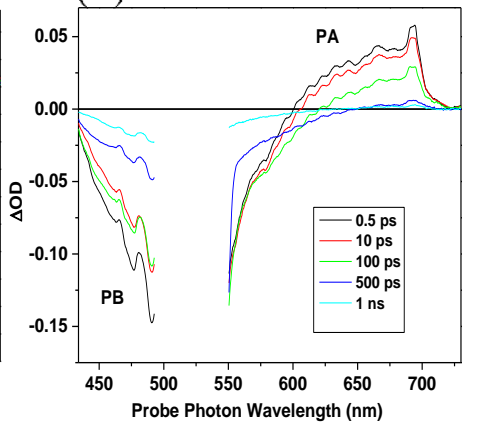

Figure 4.5. Transient absorption spectra at $\Delta \mathrm{t}=0.5 \mathrm{ps}, 10 \mathrm{ps}, 100 \mathrm{ps}, 500 \mathrm{ps}$, and $1 \mathrm{~ns}$ of (a) N719 dye in ACN and tert-butanol (1:1) solution and (b) FTO/TiO $/ \mathrm{N}^{2} 719$, (c) FTO/TiO $2 / \mathrm{APTES}_{\mathrm{N} 719}$, and (d) $\mathrm{FTO} / \mathrm{TiO}_{2} / \mathrm{APhS} / \mathrm{N} 719$ photoanodes. Two broad spectral regions, ground-state bleach (GSB) and excited-state absorption (ESA), are indicated.

scale of $100 \mathrm{ps}$ competes with the ESA process, resulting in a reduced positive signal. When attached to $\mathrm{TiO}_{2} \mathrm{NPs}$, either directly or via molecular linkers, the excited state is depopulated in picoseconds (see below) through electron injection to the $\mathrm{TiO}_{2}$ NPs. Such a fast electron injection process prevents the SE process, hence the disappearance of the buildup of the ESA signal. The exact mechanism of the SE process is still undetermined but probably involves triplet states given its relatively large time scale. It is unlikely that the negative contribution is due to GSB because excited states of isolated dye molecules in solution are rather long-lived (15-50 ns). ${ }^{140}$ 
Quantitative information on the time scales of ET processes can be derived by examining the transient decay kinetics at particular probe wavelengths, extracted from the 2D time-wavelength contour plots. Parts a-c of Figure 4.6 show the transient decay kinetics of the four samples at 470,650 , and $690 \mathrm{~nm}$ probe wavelengths. The GSB signal of the $\mathrm{FTO} / \mathrm{TiO}_{2} / \mathrm{APhS} / \mathrm{N} 719$ photoanode probed at $470 \mathrm{~nm}$ shows the fastest recovery among all four samples ( $\tau 500 \mathrm{ps})$. The other three samples have similar and longer recovery times ( $\tau$ $>1 \mathrm{~ns})$. The mechanism that leads to the faster recovery of the ground-state N719 population in the $\mathrm{FTO} / \mathrm{TiO}_{2} / \mathrm{APhS} / \mathrm{N} 719$ photoanode is undetermined but might be due to the aromatic nature of the APhS molecule. It is well-known that inclusion of $\pi$-electron systems, i.e., increasing the degree of conjugation, improves the fluorescence quantum efficiency. ${ }^{141}$ It is worth noting that although not presented in figures, the transient decay kinetics probed at $570 \mathrm{~nm}$, on the red side of the pump wavelength, are almost identical to the ones probed at $470 \mathrm{~nm}$.

The transient decay kinetics probed at 650 and $690 \mathrm{~nm}$ are shown in Figure 4.6b,c. In N719 dye solution, the relaxation of the excited-state population is slow and is preceded by a buildup process discussed previously. The decay time scale is much longer than the limit of delay time of the TAPPS system (1.5 ns). It suggests that the relaxation corresponding to these probe wavelengths is from the triplet MLCT state of the N719 dye, following a fast ISC. In the ESA signal of the three photoanodes, the buildup process is absent and the decay is much faster. It consists of a faster component with 50 ps time scale, followed by another relatively slower component with 500 ps time scale except for the 
$\mathrm{FTO} / \mathrm{TiO}_{2} / \mathrm{APhS} / \mathrm{N} 719$ photoanode with $650 \mathrm{~nm}$ probe (see below). Both components might be due to various recombination processes but from singlet and triplet states, respectively. Again, the decay is faster in the $\mathrm{FTO} / \mathrm{TiO}_{2} / \mathrm{APhS} / \mathrm{N} 719$ photoanode than the other two.
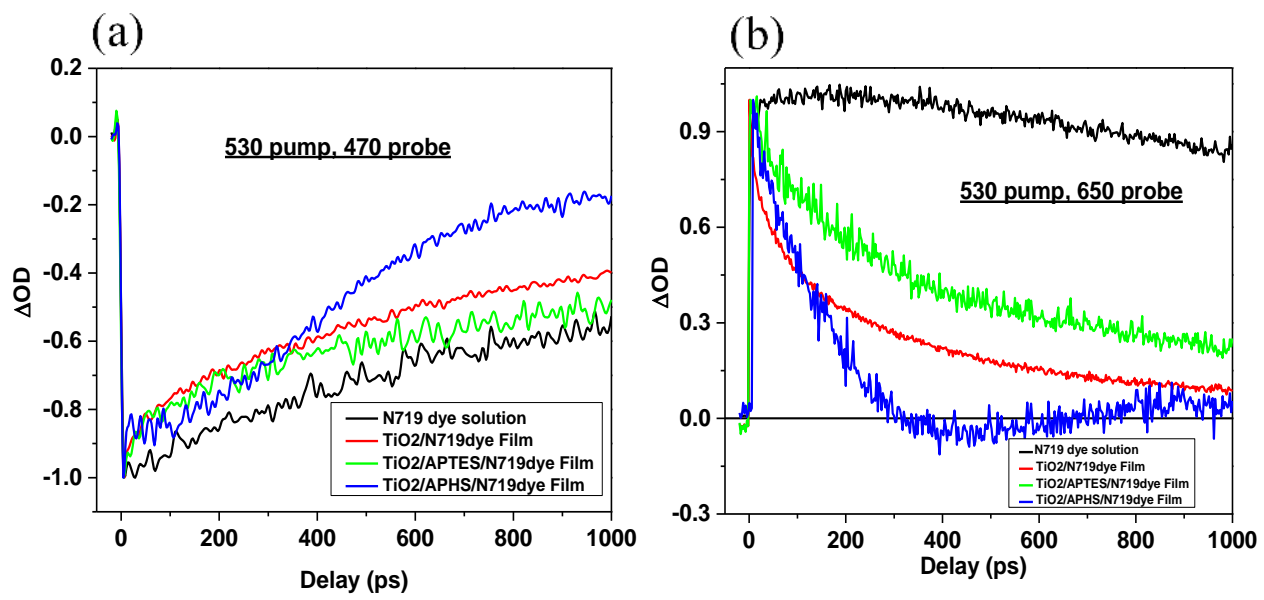

(c)

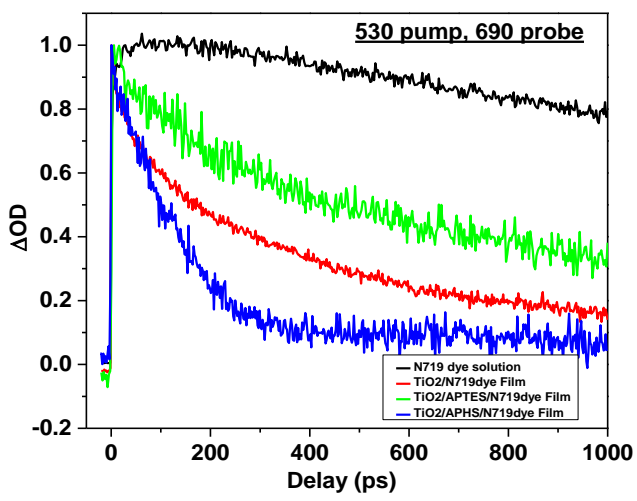

Figure 4.6. Normalized transient kinetics observed with the $530 \mathrm{~nm}$ pump wavelength and (a) $470 \mathrm{~nm}$, (b) $650 \mathrm{~nm}$, and (c) $690 \mathrm{~nm}$ probe wavelengths of N719 dye in ACN and tert-butanol (1:1) solution and $\mathrm{FTO} / \mathrm{TiO}_{2} / \mathrm{N} 719, \mathrm{FTO} / \mathrm{TiO}_{2} / \mathrm{APTES} / \mathrm{N} 719$, and $\mathrm{FTO} / \mathrm{TiO}_{2} / \mathrm{APhS} / \mathrm{N} 719$ photoanodes. The delay time step size is $3 \mathrm{ps}$.

An interesting feature was observed in the transient decay kinetics of the $\mathrm{FTO} / \mathrm{TiO}_{2} / \mathrm{APhS} / \mathrm{N} 719$ photoanode with $650 \mathrm{~nm}$ probe wavelength (Figure 4.6b). Following the first (and faster) decay component, the $\triangle \mathrm{OD}$ signal changes its sign from positive to negative at $300 \mathrm{ps}$ delay time. It changes back to positive signal after $400 \mathrm{ps}$. 
Such a sign-changing feature is not observed for the other two photoanodes. It suggests that the observed $\triangle \mathrm{OD}$ signal is a combination of ESA signal and GSB signal. The negative signal probed at $470 \mathrm{~nm}$ is dominated by GSB, whose time scale is determined by groundstate recovery. The positive signal probed at $690 \mathrm{~nm}$ is dominated by ESA, the time scale of which is determined by charge recombination. It is reasonable to postulate that combination of these processes causes the complicated decay dynamics in the $\mathrm{FTO} / \mathrm{TiO}_{2} / \mathrm{APhS} / \mathrm{N} 719$ photoanode and the sign-changing. A quantitative explanation cannot be reached without further experimental investigation. We do, however, point out that the transient decay of the $\mathrm{FTO} / \mathrm{TiO}_{2} / \mathrm{APhS} / \mathrm{N} 719$ photoanode probed at $690 \mathrm{~nm}$ reaches the turning point between the fast and slow components at $400 \mathrm{ps}$ (Figure 4.6c), when the negative $\Delta \mathrm{OD}$ signal with 650 $\mathrm{nm}$ probe reaches its minimum (Figure 4.6b). A probe delay of $400 \mathrm{ps}$ is clearly a critical time point for change in dominant photo-induced processes.

The delay time step size ( 3 ps) used to obtain the time-wavelength 2D plots in Figure 4.4 is too large to detect the ultrafast electron injection from the N719 dye. Decay dynamics in the three photoanodes were therefore monitored in TAPPS in a shorter delay time range (up to $10 \mathrm{ps)} \mathrm{with} \mathrm{the} 690 \mathrm{~nm}$ probe wavelength. The step size is $20 \mathrm{fs}$. The recorded decay dynamics and fit curves are shown in Figure 4.7. Unlike those with the $860 \mathrm{~nm}$ probe wavelength (Figure 4.4.3b-d), the decay dynamics with the $690 \mathrm{~nm}$ probe wavelength are best fit to a single exponential decay. The time constants are $2.3 \pm 0.6 \mathrm{ps}$ for $\mathrm{FTO} / \mathrm{TiO}_{2} / \mathrm{N} 719,4.4 \pm 0.2 \mathrm{ps}$ for $\mathrm{FTO} / \mathrm{TiO}_{2} / \mathrm{APTES} / \mathrm{N} 719$, and $3.3 \pm 0.4 \mathrm{ps}$ for $\mathrm{FTO} / \mathrm{TiO}_{2} / \mathrm{APhS} / \mathrm{N} 719$ photoanodes (see also Table 4.1). The time scales are comparable to those of the slower components of the decay dynamics probed at $860 \mathrm{~nm}$ and can be attributed to electron injection from the triplet states of N719 molecules to the conduction 
band of the $\mathrm{TiO}_{2}$ NPs. The rise time observed in Figure 4.6a-c therefore corresponds to both the formation and ensuing thermalization of the triplet states. The electron injection rate measured at $690 \mathrm{~nm}$ decreased upon insertion of molecular linkers. The magnitude of the decrease, however, is less significant than with $860 \mathrm{~nm}$ probe wavelength (see the

(a)

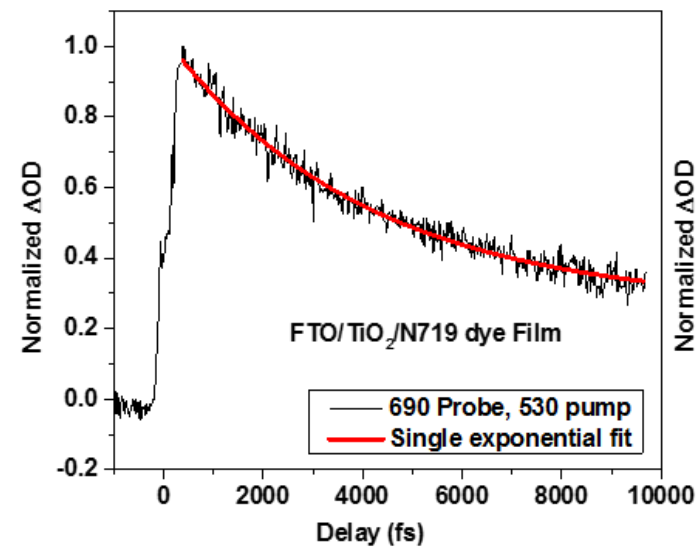

(b)

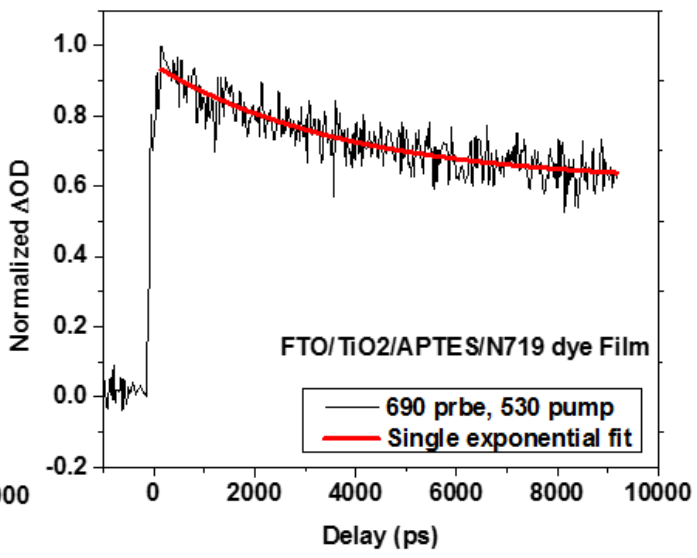

(c)

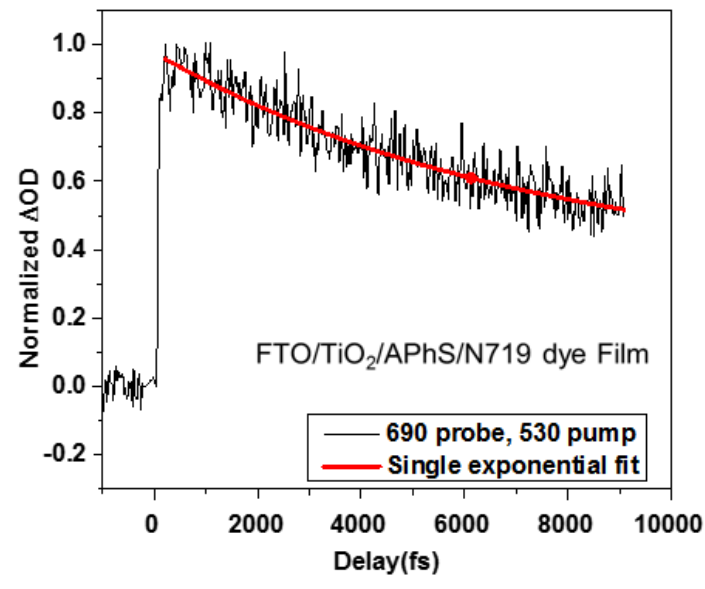

Figure 4.7. Normalized transient kinetics observed with the $530 \mathrm{~nm}$ pump wavelength and $690 \mathrm{~nm}$ probe wavelength of (a) $\mathrm{FTO} / \mathrm{TiO}_{2} / \mathrm{N} 719$, (b) $\mathrm{FTO} / \mathrm{TiO}_{2} / \mathrm{APTES} / \mathrm{N} 719$, and (c) $\mathrm{FTO} / \mathrm{TiO}_{2} / \mathrm{APhS} / \mathrm{N} 719$ photoanodes. The delay time step size is $20 \mathrm{fs}$.

Discussion). As with the $860 \mathrm{~nm}$ probe wavelength, the hindering effect of the APhS linker is weaker than for APTES. 


\section{Discussions}

For a given pair of electron donor and acceptor, the ET rate is determined by both the relative energetics at the semiconductor/sensitizing dye interface and their electronic coupling. In the present work, the electron injection process was investigated at different wavelengths for photoanodes with and without covalent molecular linkers. The linker molecules affect the electronic coupling whereas different probe wavelengths interrogate ET processes corresponding to different energetics.

The biphasic nature of the TAPP spectra of the conventional FTO/TiO $2 / \mathrm{N} 79$ photoanode observed using the $860 \mathrm{~nm}$ probe beam within a 100 ps delay time (Figure 4.3b-d) reveals two ET channels from the $\mathrm{N} 719$ molecule to the $\mathrm{TiO}_{2} \mathrm{NP}$ : from the singlet states (1MLCT) and triplet states (3MLCT) of N719. The one order of magnitude difference between time scales for the ET processes from the singlet-state and triplet-state N719 are attributed to two factors: (i) the increase of the density of acceptor states in $\mathrm{TiO}_{2}$ above the edge of its conduction band, resulting in more energy levels available to ET from the singlet excited-state N719 molecule, and (ii) the different electronic nature of singlet and triplet states in N719, resulting in different electronic couplings between the energy levels of the excited donor molecule and the acceptor semiconductor NP.

When the $690 \mathrm{~nm}$ probe wavelength is used, the TAPPS kinetics observed in the $\mathrm{FTO} / \mathrm{TiO}_{2} / \mathrm{N} 719$ photoanode can be fit to a single exponential decay with a time constant of 2.3 ps. This value is close to the time constants of the slower component in the TAPPS kinetics observed using $860 \mathrm{~nm}$ probe wavelength (1.9 ps). The decay is therefore attributed to electron injection from triplet-state N719 molecules as well, but most probably with different energetics. Electron injection from the thermalized triplet states is expected to be 
slower than that from the vibrationally excited levels of the lowest triplet state and higherenergy triplet states, both of which are generated directly by ISC. ${ }^{22}$ This is due to the different density of states in the electron acceptor, which explains the slightly longer time scale with $690 \mathrm{~nm}$ probe wavelength.

The insertion of covalently attached linker molecules (APTES and APhS) slows down the electron injection processes in all cases. Based on the Marcus theory of ET, two mechanisms may lead to the hindering effect of the molecular linker. First, the linker increases the spatial displacement between the electron donor and acceptor and hence the longer electron injection time scale. Second, the linker molecule affects the energetics for electron injection. For instance, the reorganization energy caused by the acceptor-donor interaction can be affected by the linker.

Previously, ISC on a time scale of 30 fs was reported. It is therefore puzzling that electron injection from singlet states, which occurs at much slower rates, was observed in photoanodes with covalent attachment. To explain such an observation, we note that the measured time scales are for electron injection from the dye molecules to the $\mathrm{TiO}_{2}$ nanoparticles. It is longer than the time scale of electron transfer from dye molecules to linker molecules in the covalently-attached photoanodes that is fast enough to compete with ISC. Indeed, on the basis of the fact that we were able to observe electron injection from singlet states in these photoanodes, we conclude that the time scale of electron transfer from the dye molecules to the linker molecules is similar to that of direct electron injection from dye molecules to $\mathrm{TiO}_{2} \mathrm{NPs}$ in the conventional photoanode. It is the molecular linkers that slow down the overall electron injection processes. 
The magnitude of the hindering effect depends on the decay channel, the selection of the linker molecule, and the probe wavelength. For TAPPS kinetics probed at $860 \mathrm{~nm}$, electron injection processes from both the singlet states and the triplet states of the N719 dye molecules are slowed down. For both FTO/TiO $2 /$ APTES/N719 and $\mathrm{FTO} / \mathrm{TiO}_{2} / \mathrm{APhS} / \mathrm{N} 719$ photoanodes, the hindering effect is more significant for the singletstate channel than the triplet-state channel. The different magnitudes may be attributed to different densities of accepting states in $\mathrm{TiO}_{2}$ for the two channels. Another more plausible reason is the influence of vibrational motion of the dye molecule. Due to the fast IC/IVR $(<10 \mathrm{fs})$, the electron injection for the singlet states is predominantly from the thermalized levels, whereas electron injection from the triplet states may have significant contribution from vibrationally excited states that are populated directly by ISC ( $<30 \mathrm{fs})$. It is known that molecular vibration can affect the electron injection rate. The two channels are therefore affected by the insertion of the linker molecules differently.

The hindering effect also depends on the choice of molecular linkers. The electron injection rates detected at $860 \mathrm{~nm}$ are faster $(0.3 \mathrm{ps}$ from singlet states and $7.4 \mathrm{ps}$ from triplet states) in the $\mathrm{FTO} / \mathrm{TiO}_{2} / \mathrm{APhS} / \mathrm{N} 719$ photoanode than in the $\mathrm{FTO} / \mathrm{TiO}_{2} / \mathrm{APTES} / \mathrm{N} 719$ photoanode (1.4 ps from singlet states and $16.1 \mathrm{ps}$ from triplet states). Both linkers increase the distance between the electron donor (dye sensitizer) and the acceptor $\left(\mathrm{TiO}_{2}\right)$. The APhS molecule is slightly shorter than the APTES molecule. Because the effect of linker length on the electron injection rate is exponential, the difference in linker length may lead to a considerable difference in electron injection rate. A quantitative calculation requires the determination of the damping factor, $\beta$, for the carbon chains and benzene rings and is hence not attempted here. A more significant difference between the linker molecules that explains 
the difference in electron injection rate between $\mathrm{FTO} / \mathrm{TiO}_{2} / \mathrm{APhS} / \mathrm{N} 719$ and $\mathrm{FTO} / \mathrm{TiO}_{2} / \mathrm{APTES} / \mathrm{N} 719$ photoanodes is their electronic structure: due to its aromatic nature, the molecular orbitals of $\mathrm{APhS}$ are more delocalized than APTES and hence have better overlap with the LUMO of the dye molecule and the conduction band orbitals of the NPs. The more conductive nature of the $\mathrm{APhS}$ molecules therefore leads to stronger electronic coupling between the donor and acceptor, and hence faster electron injection.

The hindering effect of the linker molecules probed at $690 \mathrm{~nm}$ is much less significant than when the $860 \mathrm{~nm}$ is used as the probe wavelength. This suggests that the hindering effect of the molecular linkers is different between different vibrational energy levels within a triplet state and probably also between different triplet states. The slower electron injection process observed with the $860 \mathrm{~nm}$ probe is from the excited triplet states formed directly by ISC, whereas that observed with $690 \mathrm{~nm}$, blue-shifted from the ESA peak, corresponds to electron injection from the thermalized and equilibrated triplet-state levels. The difference in decay time constants reflects the important role of molecular vibration in the electron injection processes. Electron injection from vibrationally hot molecules is less vulnerable to the hindering effect of the molecular linkers. This is consistent with the different magnitude in the hindering effect for the two components of the decay kinetics observed using $860 \mathrm{~nm}$ probe wavelength.

The measurement of electron injection as well as recombination processes can be affected by many other factors, for instance, the existence of multilayer dye molecules, the aggregation of the dye molecules, and different crystal orientations in the $\mathrm{TiO}_{2}$ film. On the basis of comparison between the TAPPS kinetics measured at different probe wavelengths and using different linker molecules, we conclude that such manufacturing factors do not 
affect the observed electron injection processes significantly. They can, however, alter the slower recombination processes. For instance, the $\mathrm{FTO} / \mathrm{TiO}_{2} / \mathrm{APhS} / \mathrm{N} 719$ photoanode shows faster electron recombination (Figure 4.6). Buildup of the ESA signal due to the electron injection from dye sensitizer to semiconductor NPs has been reported previously ${ }^{142-}$ ${ }^{144}$ but was not observed in the present work. It was observed for the N719 solution with 690 nm probe wavelength, which may explain the previously reported buildup in the ESA signal.

\subsection{Conclusions}

The electron injection processes were monitored in TAPPS spectroscopy. The fast and slow components of the decay kinetics with the $860 \mathrm{~nm}$ probe are attributed to electron injection from the thermalized singlet states and the vibrationally excited triplet states, respectively. When a visible white-light probe is used, in the shorter wavelength region, e.g., at $470 \mathrm{~nm}$, the signal is dominated by ground-state bleach, whereas in the longer wavelength region, e.g., at $690 \mathrm{~nm}$, electron injection from thermalized triplet states is detected. In the vicinity of the pump wavelength $(530 \mathrm{~nm})$, the signal contains contributions from both processes and is further complicated by stimulated emission.

On the basis of the TAPPS measurements, we propose that the electron injection from the excited states of $\mathrm{N} 719$ dye to $\mathrm{TiO}_{2}$ semiconductor conduction band occurs via two channels: a fast channel (tens of femtoseconds) from the singlet states of N719 and a slow channel ( 1-10 ps) from its triplet states. The electron injection process is hindered by linker molecules APTES and APhS that are covalently attached to the N719 dye molecules. The magnitude of the hindering effect depends on both the spin-multiplicity of the excited electronic states of the dye and molecular vibrations. The hindering effect is more significant 
for singlet excited-state molecules than molecules in triplet states. Electron injection from vibrationally excited triplet-state molecules is less vulnerable to the hindering effect. Electron injection processes in the $\mathrm{FTO} / \mathrm{TiO}_{2} / \mathrm{APhS} / \mathrm{N} 719$ photoanodes are faster than in the $\mathrm{FTO} / \mathrm{TiO}_{2} / \mathrm{APTES} / \mathrm{N} 719$ photoanode because the $\mathrm{APhS}$ molecule is more conductive due to its aromaticity. Suitable choice of linker molecules is therefore important in producing DSSCs that have both long stability and high PCE.

The stability of the photoanodes is significantly increased upon covalent attachment, attributed to the protective environment provided by the linker molecules to the SCN ligands. Although the molecular linkers slow the electron injection process, it is still orders of magnitude faster than the charge recombination to dye molecules and the electrolyte as well. The relatively slower electron injection rate is therefore not the reason for the observed lower PCE of the FTO/TiO $/$ APTES/N719 photoanode compared with the conventional one. The relatively lower PCE of the covalently-attached photoanodes is mainly due to the multilayer and aggregation of the dye molecules above the covalently bonded ones in addition to the possible multilayer formation of the linker itself. TAPPS results show that the $\mathrm{FTO} / \mathrm{TiO}_{2} / \mathrm{APhS} / \mathrm{N} 719$ photoanode has better coverage of monolayer dye molecules than the $\mathrm{FTO} / \mathrm{TiO}_{2} / \mathrm{APTES} / \mathrm{N} 719$ photoanode. A higher PCE is therefore expected. Monolayer coverage of linkers on semiconductor metal oxide is necessary for improving PCE of such devices, as shown recently. When the soaking time in dye solution was controlled to avoid multilayers, PCE of the FTO/TiO $/$ APTES/N719 photoanode was increased to $6.0 \pm 1.0 \%$, very close to that of the conventional one $(6.5 \pm 0.9 \%)$. Modification of the covalently-attached photoanodes, for instance, with gold nanoparticles, can further 
improve the performance of DSSCs with chemically functionalized photoanodes and will be the subject of future work. 


\section{CHAPTER 5}

\section{INCREASED EFFICIENCY OF DYE-SENSITIZED SOLAR CELLS USING A RARE EARTH OXIDE/TITANIA COMPOSITE ACCEPTOR}

Inorganic/organic surface modification of $\mathrm{TiO}_{2}$ can bring significant changes on the $\mathrm{TiO}_{2}$ nanosurface. An organic modifier, APTES, led to the covalent modification of $\mathrm{TiO}_{2}$ for linking dye strongly with $\mathrm{TiO}_{2}$. As a result, stable photoanodes were capable of being photoactive for 6 months when stored in air. Traditional photoanodes should be sealed immediately to make sandwich devices to preserve their photoactivity. The dye coverage on $\mathrm{TiO}_{2}$ plays a vital role in the PCE of devices. This chapter describes the doping of nano$\mathrm{TiO}_{2}$ with micro- $\mathrm{Nd}_{2} \mathrm{O}_{3}$ particles and evaluation of the PEC performance by $\mathrm{I}-\mathrm{V}$ curves, IPCE, and EIS techniques. The effect of dye coverage on the photophysics and photochemistry of doped and undoped photoanodes were investigated. Characterization of this thin film was performed by SEM and optical microscopy. The photoanodes were sealed using a thermal press instead of the hot plate method and the PEC values were monitored for more than one month to determine stability and quality of sealing. 


\subsection{Introduction}

DSSCs offer relatively high photo-conversion efficiencies while providing potential savings in fabrication costs compared to crystalline semiconductor photovoltaics. ${ }^{21,107,145}$ Interfacial engineering ${ }^{146}$ of the oxide material is one of the main strategies used to improve the efficiency of DSSCs ${ }^{147}$ This includes depositing a scattering layer of large size $\mathrm{TiO}_{2}$ particles on top of the active layer, ${ }^{148}$ controlling the haze of the film by incorporating larger $\mathrm{TiO}_{2}$ nanoparticles into the film, ${ }^{13,149}$ chemically modifying the surface of the active layer with molecular linkers, coating the active layer with another metal oxide to form core/shell structures, ${ }^{150,151}$ and doping the photoanodes with metal ions, ${ }^{152}$ metal nanoparticles, ${ }^{153,154}$ or other dopants ${ }^{155}$ or metal oxides. ${ }^{156}$

Rare earth metals and oxides, well known for optical materials applications, show much promise as dopants to improve $\mathrm{TiO}_{2}$ for photovoltaic applications. ${ }^{157-159}$ Rare earth doping has been observed to reduce the band-gap of $\mathrm{TiO}_{2}$ particles and increase the surface area of $\mathrm{TiO}_{2}$ nanoparticle films. ${ }^{160}$ Rare earth doping improves $\mathrm{TiO}_{2}$ photocatalytic activity and photoelectrochemical response of a $\mathrm{TiO}_{2}$ anode. ${ }^{161}$ A hybrid organic/inorganic solar cell was demonstrated in which the $\mathrm{TiO}_{2}$ was completely replaced by rare earth oxide. ${ }^{162}$ In DSSCs, a number of studies have shown that incorporation of a rare earth dopant in the photoanode can improve the cell efficiency. ${ }^{159,163-170}$ The rare earth material is usually incorporated from the rare earth salt precursor into the $\mathrm{TiO}_{2}$ nanostructures during synthesis, ${ }^{163,166,168,169,171}$ sometimes as a core/shell structure, ${ }^{169}$ but has also been incorporated by soaking a prepared $\mathrm{TiO}_{2}$ photoanode into a rare earth salt solution followed by calcination ${ }^{167}$ or by mixing rare earth nanoparticles with $\mathrm{TiO}_{2}$ nanoparticles followed by film preparation and calcination. ${ }^{165}$ Upconverting, ${ }^{172-178}$ downconverting, ${ }^{163,174,179-184}$ or 
dual-mode ${ }^{185}$ luminescent rare earth nanostructures allow improved light collection efficiency when incorporated into the $\mathrm{TiO}_{2}$ photoanode in DSSCs. They can also help improve efficiency through light scattering, ${ }^{173,177,184,186}$ altering the energy level of the $\mathrm{TiO}_{2},{ }^{177,182,187}$ and by reducing recombination processes ${ }^{181,188,189}$ at the $\mathrm{TiO}_{2} /$ electrolyte interface. Maximum efficiencies in the 9-10\% range were observed with dye-sensitized $\mathrm{TiO}_{2}$ photoanodes doped with rare earth materials. ${ }^{167,178,189}$ Qin et al. recently reported $11.2 \%$ efficiency with a perovskite-based solar cell by incorporating $0.5 \%$ Yttrium into the $\mathrm{TiO}_{2}$ layer. ${ }^{190}$ These results, while promising, suggest that further improvements might be possible through optimization of the rare earth doping concentration, material properties, and incorporation technique.

\subsection{Experimental Section}

Photoanodes were prepared according to Scheme 5.1. Briefly, fluorine doped tin oxide (FTO) coated glass slides were solvent cleaned, and then soaked in $40 \mathrm{mM} \mathrm{TiCl} 4$ solution at $70{ }^{\circ} \mathrm{C}$ for $30 \mathrm{~min}$. The slides were masked with adhesive tape to create a 0.25 $\mathrm{cm}^{2}$ window. Dyesol $\mathrm{TiO}_{2}$ paste (DSL 18 NR-T or 30NRD) was then deposited using the doctor blade method, and this process was repeated to obtain a film thickness of approximately $8.8 \mu \mathrm{m}$. Each time the film was exposed to saturated ethanol vapor in a closed box for approximately 1 minute and heated at $125^{\circ} \mathrm{C}$ for levelling of the film. In the highest efficiency cells, an additional 3-4 $\mu$ m thick scattering layer of $200-400 \mathrm{~nm} \mathrm{TiO}_{2}$ particles (WER-2) was also deposited. The paste was sintered at $500{ }^{\circ} \mathrm{C}$ for $1 \mathrm{~h}$, post treated with $\mathrm{TiCl}_{4}$ and then sintered again at $500{ }^{\circ} \mathrm{C}$ for $1 \mathrm{~h}$. For the composite electrodes, 40 mesh oxidized Nd powder (Alfa Aesar) was ground finely with the $\mathrm{TiO}_{2}$ paste in a chosen weight 
ratio and then deposited on the FTO slides in the first layer using the procedure described above. The second layer was performed with the $\mathrm{Dyesol}^{\mathrm{TiO}_{2}}$ paste only as described above. The percentage of $\mathrm{Nd}$ reported in the main text refers to the percent $\mathrm{Nd}$ in the first layer only. Sensitization of the $\mathrm{FTO} / \mathrm{TiO}_{2}$ and $\mathrm{FTO} / \mathrm{TiO}_{2}-\mathrm{Nd}_{2} \mathrm{O}_{3}$ electrodes was achieved by immersing the electrodes into a $0.3 \mathrm{mM}$ solution of Dyesol N719 dye in a 1:1 mixture of acetonitrile and $t$-butanol for $24 \mathrm{~h}$. Counter electrodes with holes made by a $0.8 \mathrm{~mm}$

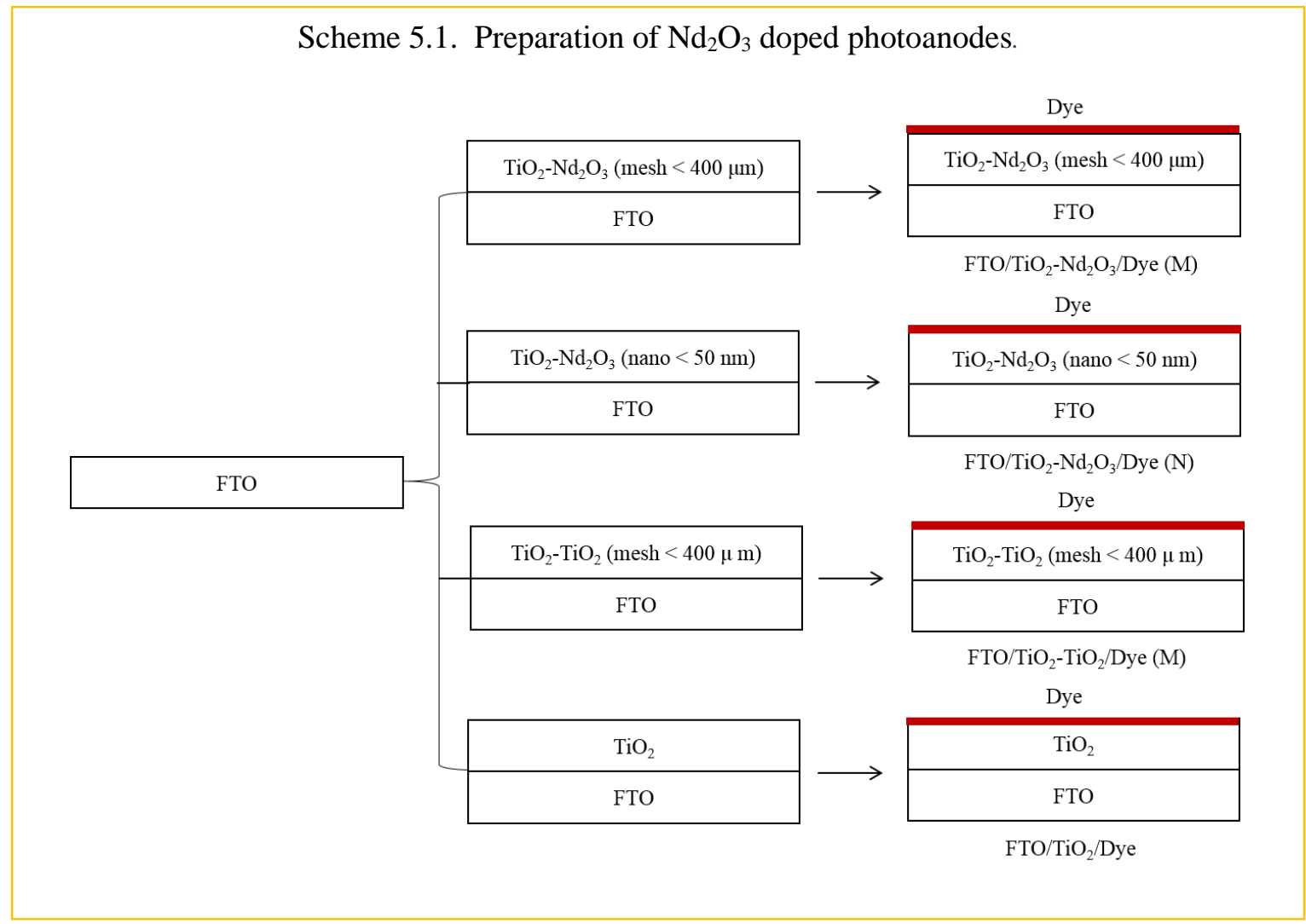

diamond tip drilling bit were prepared by coating a cleaned FTO slide with a drop of Platisol and heating to $500{ }^{\circ} \mathrm{C}$ for $20 \mathrm{~min}$. Both electrodes were painted by hot melted indium chunk from the contact to the edges to produce good electrical contact. Sandwiched cells were prepared by inserting plastic ionomer (Surlyn) between the working electrode and counter electrode and heating at $118{ }^{\circ} \mathrm{C}$ for one minute in a thermal press (model number, MPRESS912, HeatPress Nation, USA). The samples with surlyn were placed at the center 
of the floor of the thermal press and pressed by hand for 1 minute (caution: the FTO glasses should not break). For more improvement in hermetical sealing of devices, epoxy was placed all around the contact joining the photoanode and the counter electrode. A drop of electrolyte was placed on the top of the hole made in the counter electrode. If sealing was good, the drop would not go into the device because of the air pressure produced by the trapped air in between the counter electrode and photoanode. The whole device was kept in a small vacuum box, and air was pumped out for $15 \mathrm{~s}$. When air was pumped in, the electrolyte filled the vacuum space and the porous part of the dye-coated photoanode. The drilled hole was then filled with liquid Surlyn sealant and on the top of it a drop of melted Crystalbond adhesive wax (Ted Pella, USA) was applied to seal the hole. Epoxy was placed on top of the cooled wax.

Photoelectrochemical Measurements: For photoelectrochemical measurements, the cells were illuminated with simulated 1.5 AM solar light (Newport Oriel) with an intensity of $100 \mathrm{~mW} / \mathrm{cm}^{2}$ (1 Sun). The intensity of the light was set based on the voltage response of a commercially purchased, calibrated $1 \mathrm{~cm}^{2}$ reference silicon solar cell from Abet Technologies, Inc., USA with in-built KG5 filter (uncertainty $=8 \%$ ). The light source was set to 1 Sun by adjusting the light intensity until the reference silicon solar cell gave a reading of $100 \mathrm{mV}$ as measured by a voltmeter. A homemade optical sample holder was used to mount the reference silicon solar cell. After setting the light intensity, the reference silicon solar cell was replaced with the fabricated dye-sensitized solar cell (DSSC) under study in the same location at the same distance from the light source as dictated by the optical holder. The devices were masked by sticky black paper tape with an open window area of $0.16 \mathrm{~cm}^{2}$ prepared by a laser printer. Black electrical tape was used to seal all edges of the DSSC 
devices in order to prevent light piping. Current-voltage curves were obtained with a Model 273A EG \& G Princeton Applied Research Potentiostat by scanning the voltage from 0.2 V to $-1.0 \mathrm{~V}$ at a scan rate of $20 \mathrm{mV} / \mathrm{s}$ to the cells and measuring the current.

Other Characterization. Absorbance spectra of the real photoanodes were measured with a Varian Cary 50 Win UV-vis spectrophotometer and diffuse reflectance spectroscopic (DRS) measurements were performed using a Lambda 950 PerkinElmer DRS Instrument. Electrochemical Impedance Spectroscopic (EIS) measurements were performed using a CHI 660a electrochemical workstation. A $10 \mathrm{mV}$ AC perturbation at open circuit voltage was applied between the photoanode and the platinum counter electrode with a frequency range of $1000 \mathrm{kHz}$ to $5 \mathrm{mHz}$. The photoanodes were maintained at the open circuit voltage by illuminating with white light $\left(35 \mathrm{~mW} / \mathrm{cm}^{2}\right)$ produced from an optical fiber (Fiber Optic

Illuminator Model 190, Dolan-Jenner). Incident-photon-current-conversion-efficiency (IPCE, direct current) was measured using a halogen lamp with monochromatic filters ranging from $500 \mathrm{~nm}$ to $800 \mathrm{~nm}$. Incident-photon-current-conversion-efficiency (IPCE, alternating current) was also measured using the same set-up except the monochromatic light source was chopped at $13 \mathrm{~Hz}$.

\subsection{Results and Discussions}

Figure 5.1 compares images of traditional $\mathrm{TiO}_{2}$ nanoparticle films $(\mathbf{A}, \mathbf{C}$, and $\mathbf{E})$ and those created by combining $5 \%$ oxidized 40 mesh $\mathrm{Nd}$ powder with the $\mathrm{TiO}_{2}$ nanoparticles (B, D, and F). Figure 5.1(A) and (B) show optical images, while the others show scanning 
electron microscope images at increasing magnification. The $\mathrm{Nd}_{2} \mathrm{O}_{3}$ particles are clearly visible as large protrusions within the $\mathrm{TiO}_{2}$ nanoparticle film. Grinding of the mixture produces a distribution of $\mathrm{Nd}_{2} \mathrm{O}_{3}$ particles from 100s of nm up to 100 microns. Increased magnification reveals that cracks have formed near the $\mathrm{Nd}_{2} \mathrm{O}_{3}$ particles during sintering.

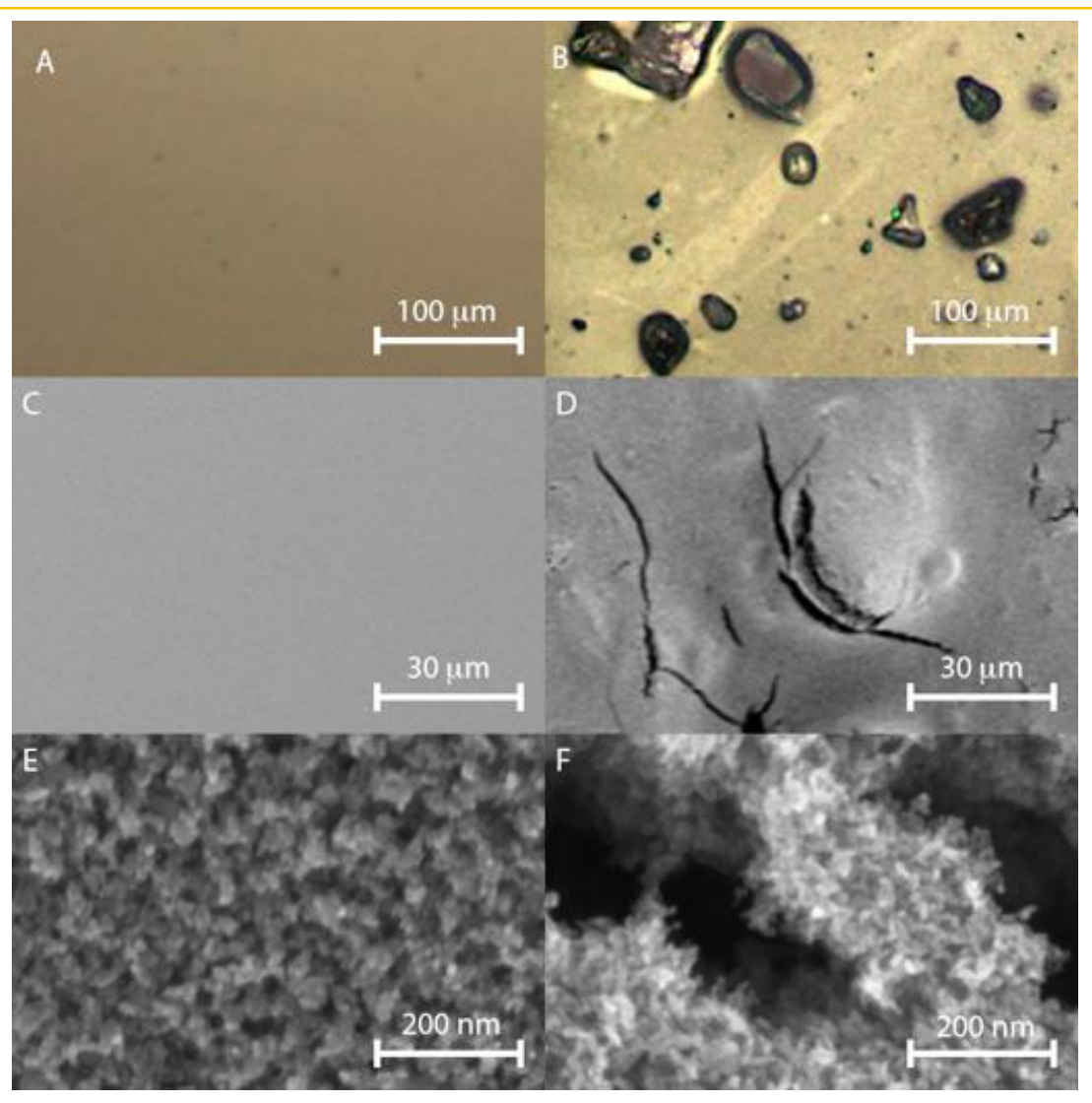

Figure 5.1. Images of the surface of $\mathrm{TiO}_{2}(\mathrm{~A}, \mathrm{C}$, and $\mathrm{E})$ and $5 \% \mathrm{Nd}_{2} \mathrm{O}_{3} / \mathrm{TiO}_{2}(\mathrm{~B}, \mathrm{D}$ and $\mathrm{F})$ photoanodes. A-B show optical microscope images while C-F show scanning electron microscope images.

Figure 5.1(D) and (F) show that the nanoscale $\mathrm{TiO}_{2}$ particles fill the cracks, along with the region on top of the $\mathrm{Nd}_{2} \mathrm{O}_{3}$ mesoparticles. Optical reflectance measurements of the $\mathrm{TiO}_{2}$ and composite electrodes (Figure 5.2) show that the $\mathrm{TiO}_{2}$ band edge is not affected by the $\mathrm{Nd}_{2} \mathrm{O}_{3}$ particles, however, additional features due to $\mathrm{Nd}_{2} \mathrm{O}_{3}$ optical transitions are 
observed. ${ }^{191}$ Peaks can be assigned as follows: 817 from ${ }^{4} \mathrm{I}_{9 / 2}$ to ${ }^{2} \mathrm{H}_{9 / 2}+{ }^{4} \mathrm{~F}_{5 / 2}, 753$ from ${ }^{4} \mathrm{I}_{9 / 2}$ to ${ }^{4} \mathrm{~F}_{5 / 2}+4 \mathrm{~S}_{3 / 2}, 690$ from ${ }^{4} \mathrm{I}_{9 / 2}$ to ${ }^{4} \mathrm{~F}_{9 / 2}, 505$ from ${ }^{4} \mathrm{I}_{9 / 2}$ to ${ }^{4} \mathrm{G}_{5 / 2}+{ }^{3} \mathrm{G}_{7 / 2}$.

Six batches of photoanodes were fabricated and characterized. Each batch contains both standard $\mathrm{TiO}_{2}$ photoanodes and photoanodes doped with $5 \%$ oxidized 40 mesh $\mathrm{Nd}$ powder. Following measurements, results from multiple (from 2 to 4 ) devices of the same type in a

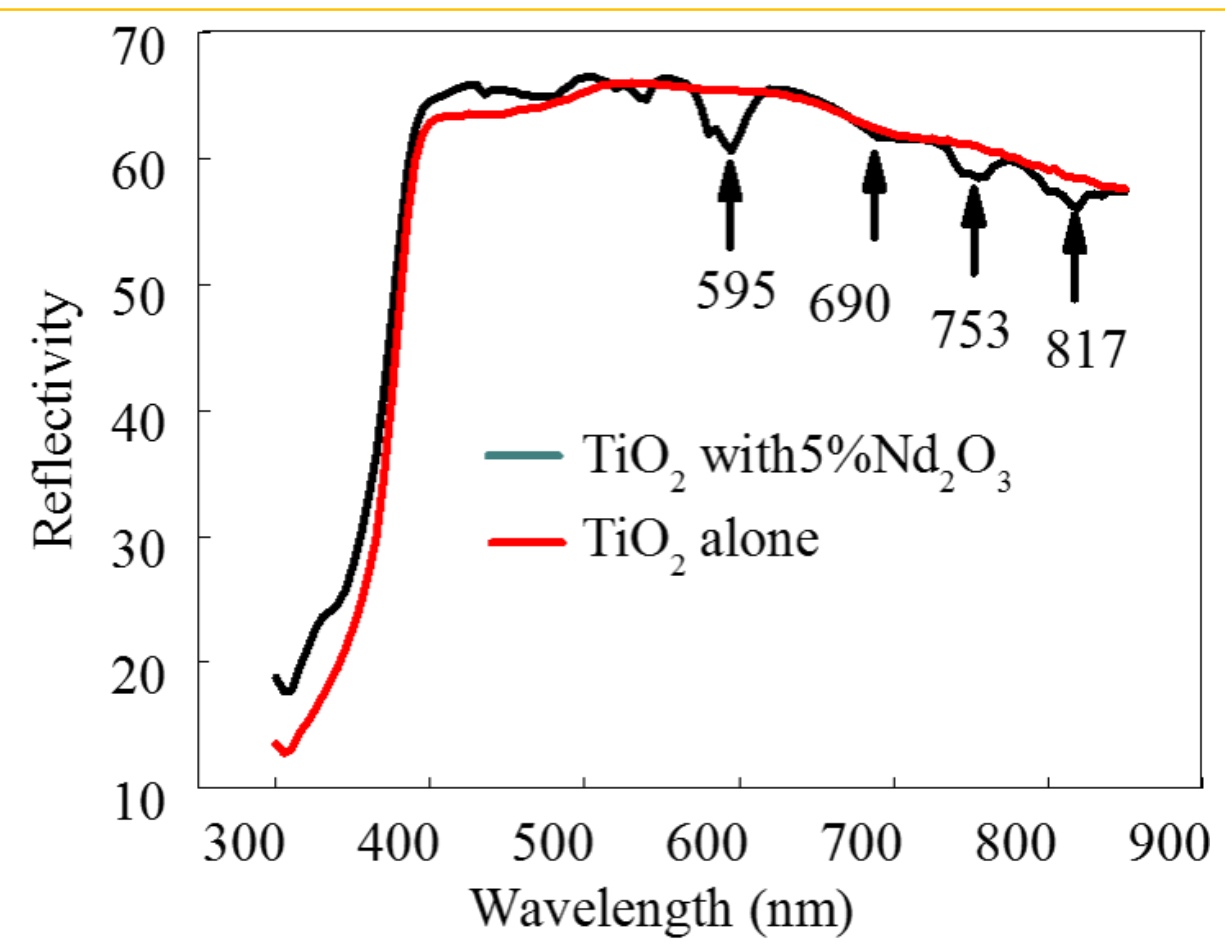

Figure 5.2. Optical reflectance measurements of a $\mathrm{TiO}_{2}$ film (red) and a $\mathrm{TiO}_{2}$ film doped with $5 \% \mathrm{Nd}_{2} \mathrm{O}_{3}$ microparticles (blue). The band edge is similar for both films and the transitions for the $\mathrm{Nd}_{2} \mathrm{O}_{3}$ are clearly observed in the visible range for the hybrid film as noted by the arrows.

single batch are averaged together. Figures 5.3A and 5.3B show the current density versus bias for the $\mathrm{Nd}_{2} \mathrm{O}_{3}$ doped devices and undoped devices, respectively. Each color corresponds to a different batch (the results from batch 2 are left out for clarity). The voltage axis is reversed between the two plots to allow for easier comparison of the data. There is considerable variation in the current-voltage characteristics between different batches of 
solar cells. However, for each batch, the short circuit current is substantially higher for the samples containing the $\mathrm{Nd}_{2} \mathrm{O}_{3}$ than for the standard DSSCs. Figures 5.3 C-F are box plots summarizing the photoelectrochemical parameters for the $\mathrm{Nd}_{2} \mathrm{O}_{3}$-doped (solid boxes) and
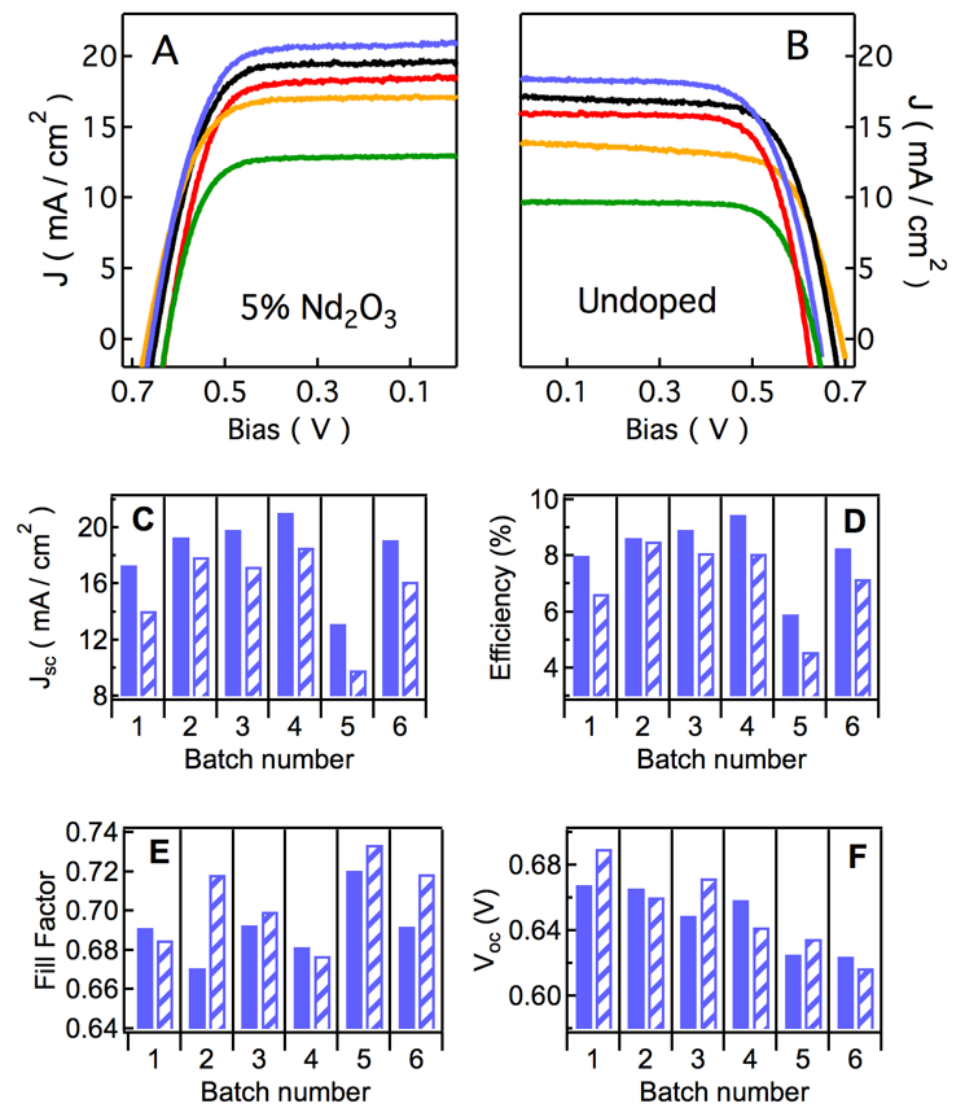

Figure 5.3. Current-density versus bias for (A) $\mathrm{Nd}_{2} \mathrm{O}_{3}$ doped and (B) undoped photoanodes measured under 1.5 AM illumination. Different batches are shown as different colored lines: Batch 1(yellow), 2 (not shown), 3 (black), 4 (blue), 5 (green) and 6 (red). (C-F) Box plots show (C) short circuit current, (D) efficiency, (E) fill factor, and (F) open circuit voltage for each of the six batches. Solid blocks show $\mathrm{Nd}_{2} \mathrm{O}_{3}$ doped samples, and hashed blocks show undoped samples.

undoped (hashed boxes) photoanodes for all six batches. As shown in Figure 5.3C (and described above) the short circuit current density is higher for the $\mathrm{Nd}_{2} \mathrm{O}_{3}$ doped samples than for the undoped samples in each batch. This produces the observed efficiency increase shown in Figure 5.3D; the improvement in efficiency due to $\mathrm{Nd}_{2} \mathrm{O}_{3}$ doping ranges from 
about 10 to 30\%. Changes in the fill factor (Figure 5.2E) and open circuit voltage (Figure 5.2F) are small and inconsistent among the different batches, suggesting that the $\mathrm{Nd}_{2} \mathrm{O}_{3}$ has little impact on these parameters. Table 5.1 summarizes the photoelectrochemical measurements and dark current onset voltage for all of the batches of $\mathrm{Nd}_{2} \mathrm{O}_{3}$ doped and undoped devices. The dark current onset voltage varies little between the doped and undoped

\begin{tabular}{|c|c|c|c|c|c|c|c|c|c|c|c|}
\hline \multicolumn{6}{|c|}{ FTO/TiO $2-5 \% \mathrm{Nd}_{2} \mathrm{O}_{3} /$ Dye } & \multicolumn{6}{|c|}{ FTO/TiO ${ }_{2} /$ Dye } \\
\hline B.N. & $J_{s c}\left(m A c m^{-2}\right)$ & $V_{o c}$ & $\% \eta$ & $\mathrm{FF}$ & $V_{\text {dark }}(\mathrm{V})$ & B.N. & $\mathrm{J}_{s c}\left(\mathrm{mAcm}^{-2}\right)$ & $\mathrm{V}_{o c}$ & $\% n$ & $\mathrm{FF}$ & $\mathrm{V}_{\text {dark }}(\mathrm{V})$ \\
\hline \multirow[t]{2}{*}{1} & 18.56 & 0.66 & 8.63 & 0.71 & 0.48 & \multirow[t]{2}{*}{1} & 13.82 & 0.69 & 6.64 & 0.70 & NM \\
\hline & 15.75 & 0.69 & 7.46 & 0.69 & 0.44 & & $\ldots$ & $\ldots$ & $\ldots$ & $\ldots$ & $\ldots$ \\
\hline \multirow[t]{3}{*}{2} & 20.06 & 0.68 & 9.42 & 0.69 & 0.46 & \multirow[t]{3}{*}{2} & 17.75 & 0.68 & 8.51 & 0.70 & 0.48 \\
\hline & 18.25 & 0.67 & 8.39 & 0.69 & NM & & $\ldots$ & $\ldots-$. & -.-- & $\ldots$ & $\ldots$ \\
\hline & 19.56 & 0.66 & 8.24 & 0.64 & NM & & $\ldots$ & $\ldots$ & $\cdots$ & $\ldots$ & $\ldots$ \\
\hline 3 & 19.63 & 0.65 & 8.94 & 0.70 & 0.55 & 3 & 17.19 & 0.67 & 8.11 & 0.70 & 0.44 \\
\hline \multirow[t]{2}{*}{4} & 21.75 & 0.66 & 9.86 & 0.69 & NM & \multirow[t]{2}{*}{4} & 17.19 & 0.65 & 7.26 & 0.65 & NM \\
\hline & 19.94 & 0.66 & 9.16 & 0.70 & NM & & 19.75 & 0.64 & 9.00 & 0.72 & NM \\
\hline \multirow[t]{3}{*}{5} & 13.88 & 0.61 & 6.16 & 0.73 & 0.48 & 5 & 9.73 & 0.64 & 4.59 & 0.74 & 0.56 \\
\hline & 15.69 & 0.64 & 7.37 & 0.73 & 0.54 & & $\ldots$ & $\ldots$ & $\ldots$ & $\ldots$ & $\ldots$ \\
\hline & 9.56 & 0.63 & 4.36 & 0.73 & 0.52 & & $\ldots$ & $\ldots$ & $\cdots$ & $\ldots$ & $\ldots$ \\
\hline 6 & 18.50 & 0.64 & 8.26 & 0.70 & 0.55 & 6 & 15.88 & 0.62 & 7.18 & 0.73 & 0.52 \\
\hline
\end{tabular}

samples, suggesting that recombination kinetics are similar in both types of photoanodes.

Further experiments show that the $\mathrm{Nd}_{2} \mathrm{O}_{3}$ doping does not negatively impact the device stability as long as it is sealed properly. Figure $\mathbf{5 . 4}$ shows cyclic voltammetry results for conventional and $\mathrm{Nd}_{2} \mathrm{O}_{3}$ doped samples after 50 cycles of scan were performed at a rate of $20 \mathrm{mV} / \mathrm{s}$ over a voltage window of 0.0 to $1.0 \mathrm{~V}$. Both devices did not show any changes in the positive and negative scans. The devices were also scanned after 4 hours and the results were the same (not shown here). Cyclic voltammetry can be performed to test the stability and quality of the devices prepared on the same day. Some devices degraded after one hour or after one day when not sealed well. However, for some well-sealed $\mathrm{Nd}_{2} \mathrm{O}_{3}$ doped samples, there was little change in the short circuit current and efficiency for up to 40 days (Figure 5.5). 
Photoelectrochemical measurements show that the efficiency of DSSCs with photoanodes doped with $\mathrm{Nd}_{2} \mathrm{O}_{3}$ is strongly dependent on the concentration of $\mathrm{Nd}_{2} \mathrm{O}_{3}$ by
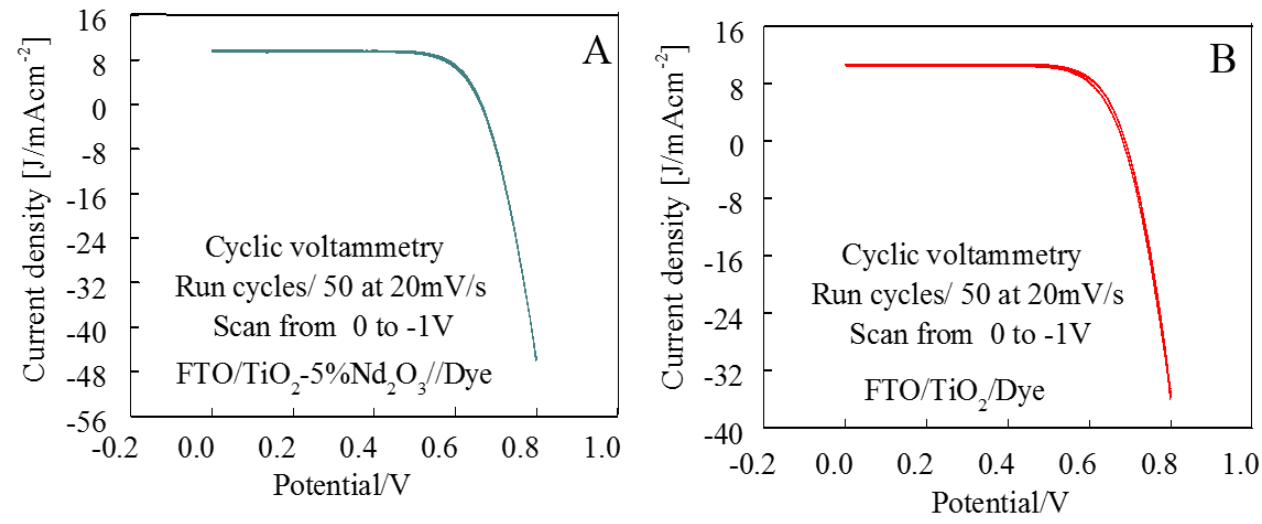

Figure 5.4. Cyclic voltammetry of (A) FTO/TiO $2-5 \% \mathrm{Nd}_{2} \mathrm{O}_{3} / \mathrm{Dye}$, and (B) FTO/TiO $/$ /Dye. The devices were illuminated by $35 \mathrm{~mW} / \mathrm{cm} 2$ optical fiber light. The 50 scans at $20 \mathrm{mV} / \mathrm{s}$ were performed by using two terminal contacts. The scan window of the voltage was from 0 to $-1 \mathrm{~V}$. The sign of the photocurrent and photovoltage are reversed in the plot.

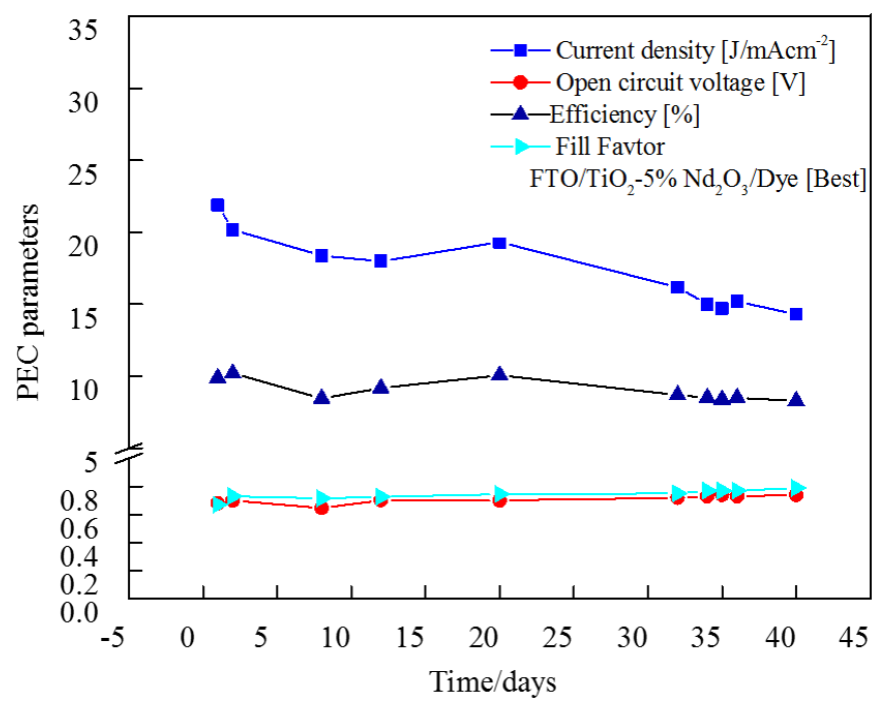

Figure 5.5. Photoelectrochemical parameters measured over a time period of 40 days of a wellsealed dye-sensitized solar cell device with a $\mathrm{FTO} / \mathrm{TiO}_{2}-5 \% \mathrm{Nd}_{2} \mathrm{O}_{3} /$ Dye photoanode.

weight relative to the $\mathrm{TiO}_{2}$. In Figure 5.6, the current density versus bias is plotted for samples containing varying weight concentrations of $\mathrm{Nd}_{2} \mathrm{O}_{3}$ under $1.5 \mathrm{AM}$ solar light. Both $5 \%$ and $20 \% \quad \mathrm{Nd}_{2} \mathrm{O}_{3}$ concentration samples have higher efficiency than the $\mathrm{TiO}_{2}$ only sample, while above $20 \%$ the efficiency is reduced. The inset shows a plot of the efficiency 
as a function of the weight percentage of $\mathrm{Nd}_{2} \mathrm{O}_{3}$ mesh particles, indicating that the maximum efficiency occurs at $\sim 5 \%$ by weight.

To better understand the improved efficiency, optical absorbance measurements were performed on the composite and standard anode materials after soaking in dye for 24 hours.

Figure 5.9A shows the resulting absorbance spectra. Both $\mathrm{TiO}_{2}$ and $\mathrm{TiO}_{2}-5 \% \mathrm{Nd}_{2} \mathrm{O}_{3}$

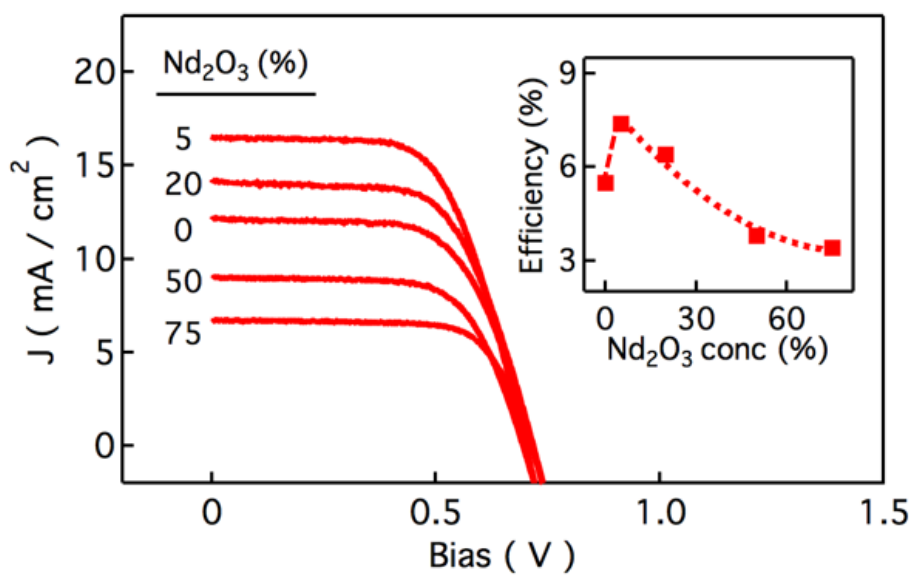

Figure 5.6. Current voltage characteristics for DSSCs made with anodes containing varying percent weight concentrations of $\mathrm{Nd}_{2} \mathrm{O}_{3}$. Inset: Efficiency as a function of $\mathrm{Nd}_{2} \mathrm{O}_{3}$ percent weight concentration.

samples show similar absorbance peaks due to the dye. The metal-to-ligand charge transfer (MLCT) band of the N719 dye appears at $538 \mathrm{~nm}$ in the pure $\mathrm{TiO}_{2}$ sample and at $544 \mathrm{~nm}$ in the sample with $5 \% \mathrm{Nd}_{2} \mathrm{O}_{3}$, a small red shift. The main influence of the $\mathrm{Nd}_{2} \mathrm{O}_{3}$ incorporation is to increase the magnitude of the dye absorbance by approximately $28 \%$, indicating that the $5 \% \mathrm{Nd}_{2} \mathrm{O}_{3} / \mathrm{TiO}_{2}$ hybrid film is able to uptake appreciably more dye than the standard sample. As shown in Figure 5.6 (inset), the increase in efficiency is maximized for 5\% $\mathrm{Nd}_{2} \mathrm{O}_{3}$ concentration, similar to the dependence on absorbance as shown in Figure 5.7. Figure 5.9B shows the magnitude of absorbance of the MLCT band as a function of dye soaking time for both $5 \% \mathrm{Nd}_{2} \mathrm{O}_{3}$ and pure $\mathrm{TiO}_{2}$. The absorption for the $5 \% \mathrm{Nd}_{2} \mathrm{O}_{3}$ sample 
rapidly increases above the pure $\mathrm{TiO}_{2}$ sample, reaching a maximum after approximately 5 hours of soaking time. The same behavior occurred reproducibly for 3 samples of $5 \% \mathrm{Nd}_{2} \mathrm{O}_{3}$ and 3 samples of pure $\mathrm{TiO}_{2}$.

As shown in Figure 5.1, the composite anodes have a distribution of particle sizes

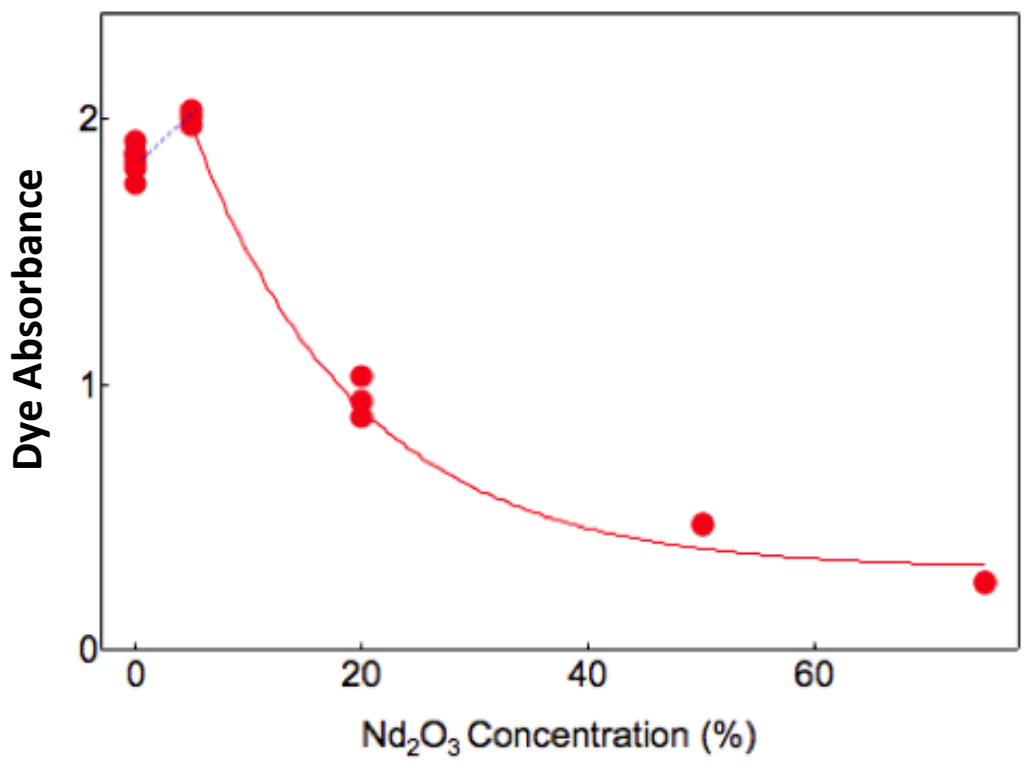

Figure 5.7. A plot of dye absorbance as a function of $\mathrm{Nd}_{2} \mathrm{O}_{3} \%$ concentration for $\mathrm{TiO}_{2}$ films doped with $\mathrm{Nd}_{2} \mathrm{O}_{3}$ microparticles. The dye absorbance, or coverage, reaches a maximum at $5 \%$ doping by weight (before calcination). The efficiency of the photoanode is largest at the same percentage.

resulting in cracks forming that fill with nanoparticles following sintering. It is possible that the cracked surface of the anode results in a larger surface area, and is able to absorb a larger quantity of dye than a more uniform surface. To test this hypothesis, anodes were made that contained 400 micron scale $\mathrm{TiO}_{2}$ particles mixed with the $\mathrm{TiO}_{2}$ nanoparticles. As shown in Figure 5.8B, the surface of the all $\mathrm{TiO}_{2}$ composite electrode appears very similar to the surface of the $5 \% \mathrm{Nd}_{2} \mathrm{O}_{3}-\mathrm{TiO}_{2}$ electrode, with cracks forming around the larger particles. However, there is an insignificant increase in the MLCT band absorbance of the $\mathrm{TiO}_{2}$ composite electrode compared with the $\mathrm{TiO}_{2}$ nanoparticles electrode (Figure 5.8C) and the 
efficiency of DSSCs made from the $\mathrm{TiO}_{2}$ composite electrodes is virtually identical to those made from the $\mathrm{TiO}_{2}$ nanoparticles electrodes (Figure 5.8D). This shows that the ability to adsorb large amounts of dye is specific to rare earth oxide incorporation. It also shows that
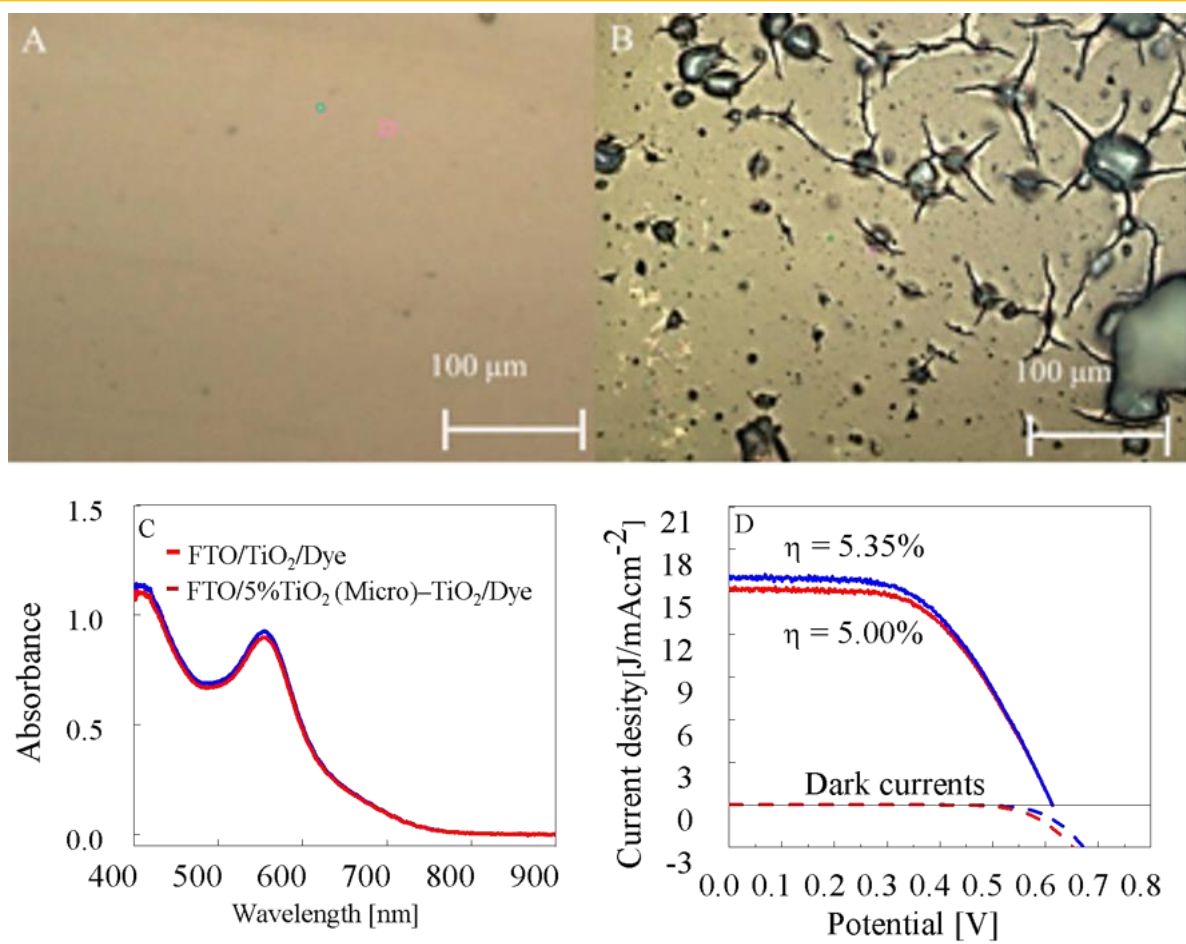

Figure 5.8. Optical images of (A) nanoscale $\mathrm{TiO}_{2}$ only and (B) nanoscale $\mathrm{TiO}_{2}$ containing $5 \%$ microscale $\mathrm{TiO}_{2}$ particles. (C) Dye absorbance on nanoscale $\mathrm{TiO}_{2}$ only (red) and nano $\mathrm{TiO}_{2}+$ $5 \%$ micro $\mathrm{TiO}_{2}$ (blue) films. (D) $\mathrm{J}-\mathrm{V}$ curves under $1.5 \mathrm{AM}$ solar light showing the efficiency of the dye-coated nano $\mathrm{TiO}_{2}$ films (solid red) and nano $\mathrm{TiO}_{2}+5 \%$ micro $\mathrm{TiO}_{2}$ (solid blue) films with the dark $\mathrm{J}-\mathrm{V}$ curves shown as the corresponding dashed lines.

the mesoscale particles of $\mathrm{TiO}_{2}$ intermixed in the cathode do not function as an effective scattering layer ${ }^{148}$ or improve the efficiency based on increased haze $e^{13,149}$ in our case, making it possible to discount these explanations for the improved efficiency in the $\mathrm{Nd}_{2} \mathrm{O}_{3}$ composite samples. Others have reported improved efficiency when mixing larger particles or powders of $\mathrm{TiO}_{2}$ of various shapes and sizes with nanoscale $\mathrm{TiO}_{2}$ nanoparticles, ${ }^{192-194}$ which has been attributed to increased dye loading, improved light collection, and improved 
electron transport, but we did not observe significant improvements for the addition of 5\% microscale $\mathrm{TiO}_{2}$ under our experimental conditions.

To determine whether the efficiency increase is solely due to increased dye uptake, solar cells were prepared using reduced dye soaking times. Using the data in Figure 5.9B as a guide, $5 \% \mathrm{Nd}_{2} \mathrm{O}_{3}$ anodes were soaked in dye for 2 hours, while pure $\mathrm{TiO}_{2}$ anodes were
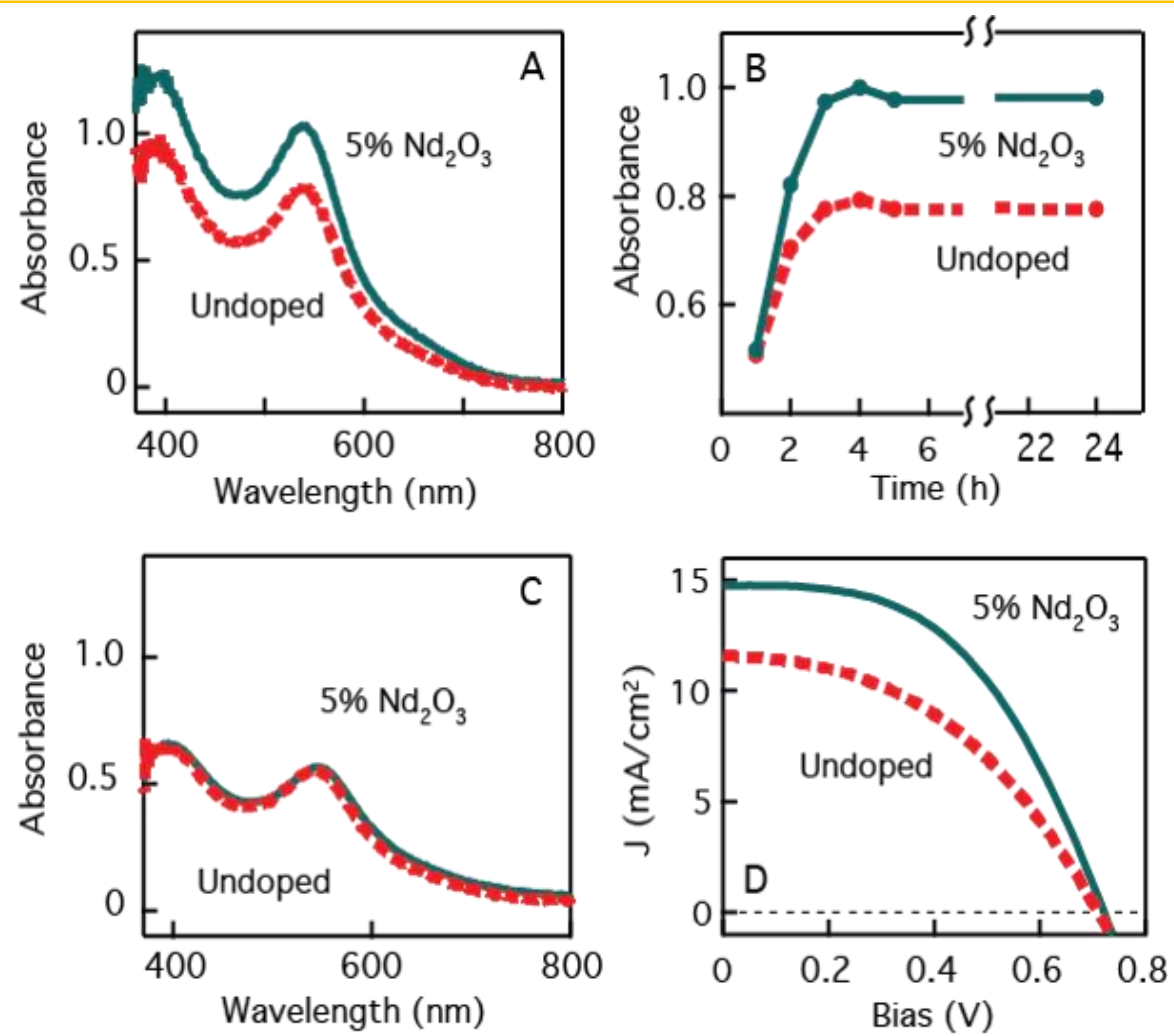

Figure 5.9. (A) UV-vis absorbance spectra for anodes made with $5 \% \mathrm{Nd}_{2} \mathrm{O}_{3} / \mathrm{TiO}_{2}$ composite anodes (green solid line) and undoped $\mathrm{TiO}_{2}$ anodes (red dashed line). (B) Absorbance as a function of dye soaking time. (C) Absorbance for reduced dye soaking time. (D) Light current voltage characteristics for the dye coverage corresponding to $(\mathrm{C})$.

soaked for 2.5 hours. The resulting absorbance spectra for the two anodes are shown in

Figure 5.9C. In this case, the MLCT band absorbance is virtually identical for the two different anodes, showing that the dye coverages should also be the same. Figure 5.9D plots the light current density versus bias curves for the two anodes with the same dye coverages. In both cases, the efficiency is appreciably lower than that observed for full dye coverage. 
However, the $5 \% \mathrm{Nd}_{2} \mathrm{O}_{3}$ anode still produces a higher efficiency (5.39\%) than the pure $\mathrm{TiO}_{2}$ anode (3.65\%). These experiments show that the $\mathrm{Nd}_{2} \mathrm{O}_{3}$ mesoparticles both increase the dye uptake and improve the charge collection and transport through the photoanode. To further investigate the charge transport within the cell, we used electrochemical impedance spectroscopy (EIS). Figure 5.10 shows the EIS Nyquist plots for the same photoanodes whose J-V plots are shown in Figure 5.6. In each plot there are three distinct semi-circles.

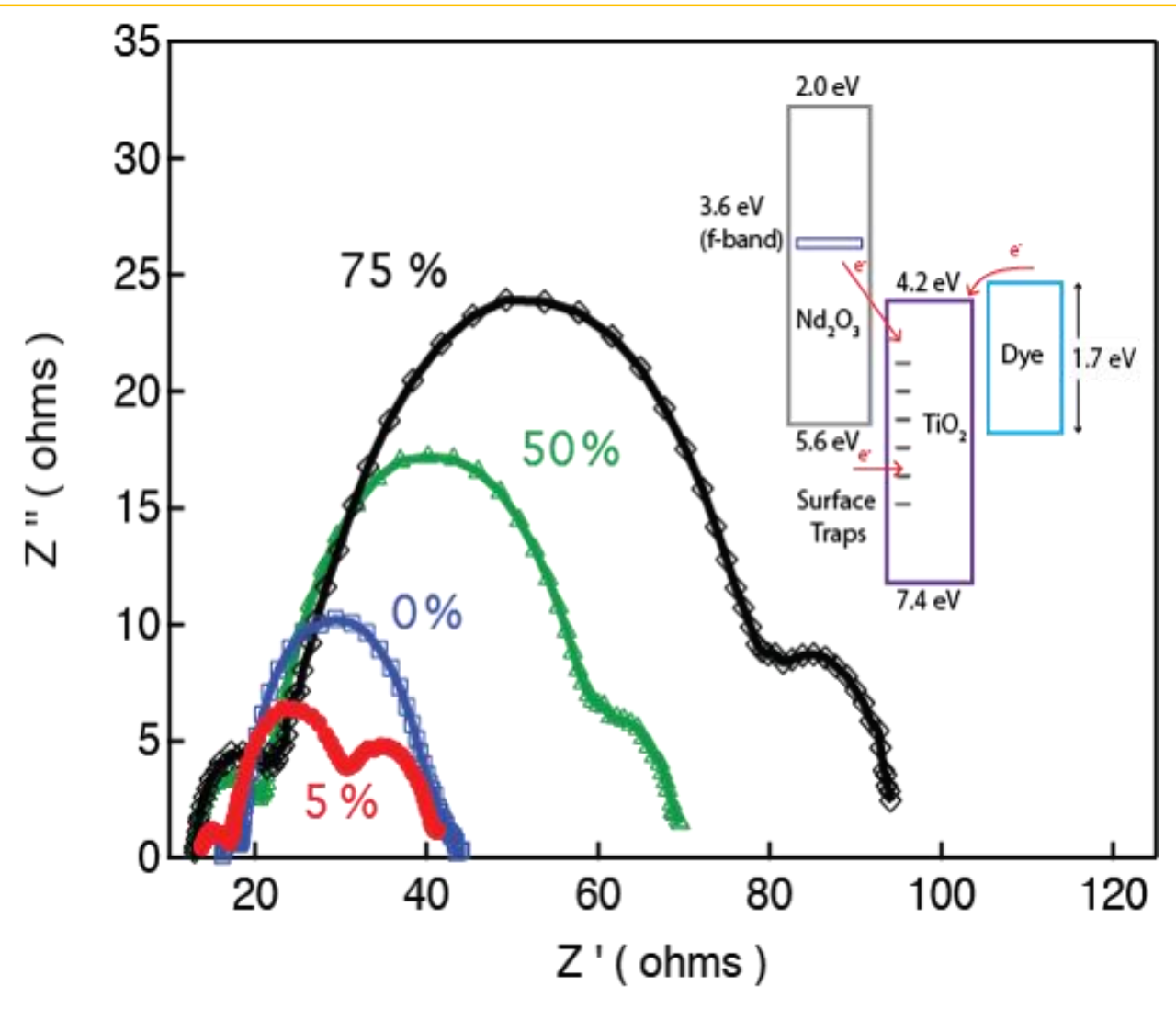

Figure 5.10. Electron impedance spectra for photoanodes containing different concentrations of $\mathrm{Nd}_{2} \mathrm{O}_{3}$ in the photoanodes.

The left semi-circle denotes the high frequency window $(100 \mathrm{kHz}-500 \mathrm{~Hz})$ and measures charge transfer from the counter electrode to the electrolyte. The middle semi-circle corresponds to the medium frequency window $(100 \mathrm{~Hz}-1 \mathrm{~Hz})$, and measures the $\mathrm{TiO}_{2} /$ Dye/electrolyte or $\mathrm{TiO}_{2}-\mathrm{Nd}_{2} \mathrm{O}_{3} /$ Dye/electrolyte interface. The right semicircle 
corresponds to the low frequency window $(1 \mathrm{~Hz}-5 \mathrm{mHz})$ and is normally considered to measure diffusion of the electrolyte/redox couple. ${ }^{195,196}$ In each case, the larger the diameter of the semicircle, the larger the charge transfer resistance. As seen in the figures, the smallest diameter semi-circle is observed for $5 \% \mathrm{Nd}_{2} \mathrm{O}_{3}$ followed by $0 \% \mathrm{Nd}_{2} \mathrm{O}_{3}, 50 \% \mathrm{Nd}_{2} \mathrm{O}_{3}$, and finally $75 \% \mathrm{Nd}_{2} \mathrm{O}_{3}$. The charge transfer resistance at the $\mathrm{TiO}_{2}-\mathrm{Nd}_{2} \mathrm{O}_{3} /$ Dye/electrolyte interface is thus lowest for the $5 \% \mathrm{Nd}_{2} \mathrm{O}_{3}$, which in turn improves the efficiency of the device. $\mathrm{OH}$ sites in $\mathrm{TiO}_{2}$ are considered as one reason for the increase in resistance in $\mathrm{TiO}_{2}$ nanoparticles. The ATR-FTIR data in Figure 5.11 of $5 \% \mathrm{Nd}_{2} \mathrm{O}_{3}$ - doped $\mathrm{TiO}_{2}$ illustrates that the $\mathrm{OH}$ sites, indicated by the $3600 \mathrm{~cm}^{-1}$ band, disappeared. Furthermore, Figure $\mathbf{5 . 1 2 A}$ illustrates the electrochemistry data where a CV was run from 0 to $-1 \mathrm{~V}$ at scan rate of 10 $\mathrm{mV} / \mathrm{s}$ in $\mathrm{KCl}$ solution. The charging current due to trap states became flat in the case of the Nd-modified electrodes. The reason and total understanding the phenomenon is still unclear. The normalized photovoltage decay curves for doped and undoped electrodes clearly shows that electron life time is short for the doped samples, meaning a better charge transfer charge transfer form conduction band to electrolyte (KSCN) in this case.

To understand how the $\mathrm{Nd}_{2} \mathrm{O}_{3}$ might reduce the charge transfer resistance, consider the simple energy diagram of the $\mathrm{Dye} / \mathrm{TiO}_{2} / \mathrm{Nd}_{2} \mathrm{O}_{3}$ system in the inset to Figure 5.10. The $\mathrm{Nd}_{2} \mathrm{O}_{3}$ valence band edge (filled $\mathrm{f}$ and spd states) has been calculated to lie well within the $\mathrm{TiO}_{2}$ band gap, while the conduction band (spd states), and empty f-band lie above the band edge of the dye LUMO level. ${ }^{197-199}$ It is unlikely that the $\mathrm{Nd}_{2} \mathrm{O}_{3}$ would provide an additional transport path for the photo excited electrons from the dye since the conduction band energy is too high, and the f-states, while available, are localized states with high transport resistance. However, it is known that $\mathrm{TiO}_{2}$ contains mid-gap surface trapping states that 
capture injected charge from the dye and lower the efficiency. It is possible that the $\mathrm{Nd}_{2} \mathrm{O}_{3}$ acts to neutralize these states through charge transfer from photoexcited states (corresponding to the transitions shown in Figure 5.10), or from the $\mathrm{Nd}_{2} \mathrm{O}_{3}$ valence band.

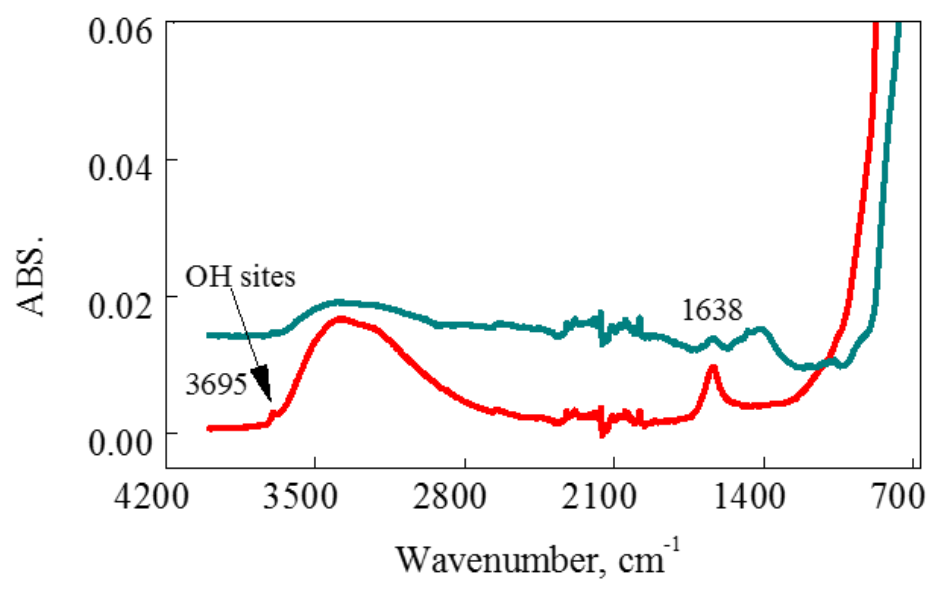

Figure 5.11. ATR-FTIR spectra of $\mathrm{FTO} / \mathrm{TiO}_{2}-5 \% \quad \mathrm{Nd}_{2} \mathrm{O}_{3}$ (dark cyan) and $\mathrm{FTO} / \mathrm{TiO}_{2}$ (red) photoanodes. $\mathrm{OH}$ site peak at $3695 \mathrm{~cm}^{-1}$ disappeared and $\mathrm{H}_{2} \mathrm{O}$ adsorbed mode is diminished in FTO/ $\mathrm{TiO}_{2}-5 \% \mathrm{Nd}_{2} \mathrm{O}_{3}$ photoanodes.
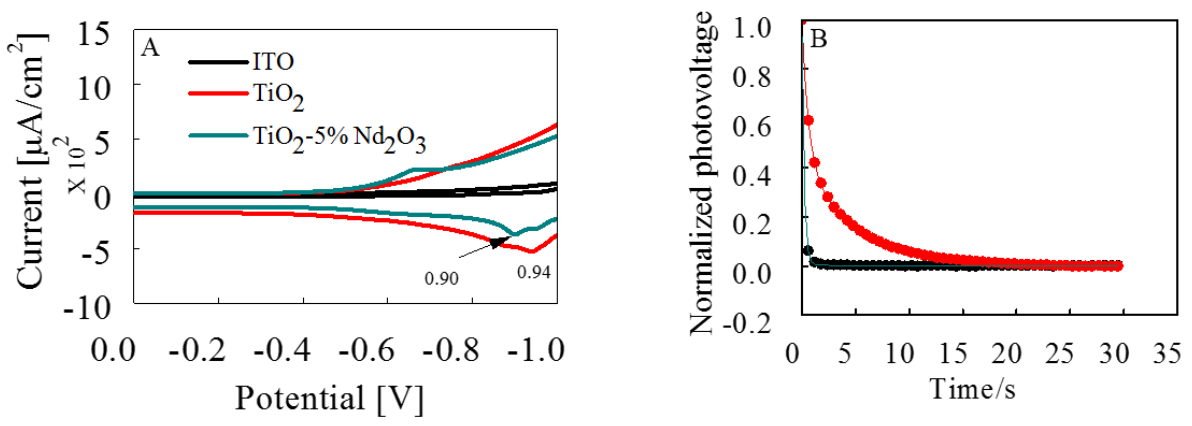

Figure 5.12. (A) Cyclic Voltammograms (CV) of ITO, ITO/TiO 2 , and $\mathrm{ITO} / \mathrm{TiO}_{2}-5 \% \mathrm{Nd}_{2} \mathrm{O}_{3}$ in $0.1 \mathrm{M} \mathrm{KCl}$ solution at pH6. (B) Normalized photovoltage $\left(\mathrm{V}_{\mathrm{ph}}\right)$ decay curve of $\mathrm{FTO} / \mathrm{TiO}_{2}$ (red), and $\mathrm{FTO} / \mathrm{TiO}_{2}-5 \% \mathrm{Nd}_{2} \mathrm{O}_{3}$ (dark cyan) anodes in a beaker cell with three terminals in $0.5 \mathrm{M} \mathrm{KSCN}$ aqueous solution.

Injected electrons from the photo excited dye can then transfer through the $\mathrm{TiO}_{2}$ without being trapped, since the $\mathrm{TiO}_{2}$ trap states are occupied. ${ }^{200}$

In order to explore the reasons for high current density produced by doped samples,

IPCE measurements were performed for optimized photoanodes but without scattering layers. The scattering layer was not used, which made it easier for measuring dye coverage 
by utilizing transmission UV-vis spectroscopy. The current and efficiency will have a lower value than that of PAs with a scattering layer. Figures 5.13A-C shows the UV-vis absorption spectra, IPCE plots, and I-V curves of doped/undoped photoanodes made in a similar environment and every measurement was recorded immediately. The IPCE of doped

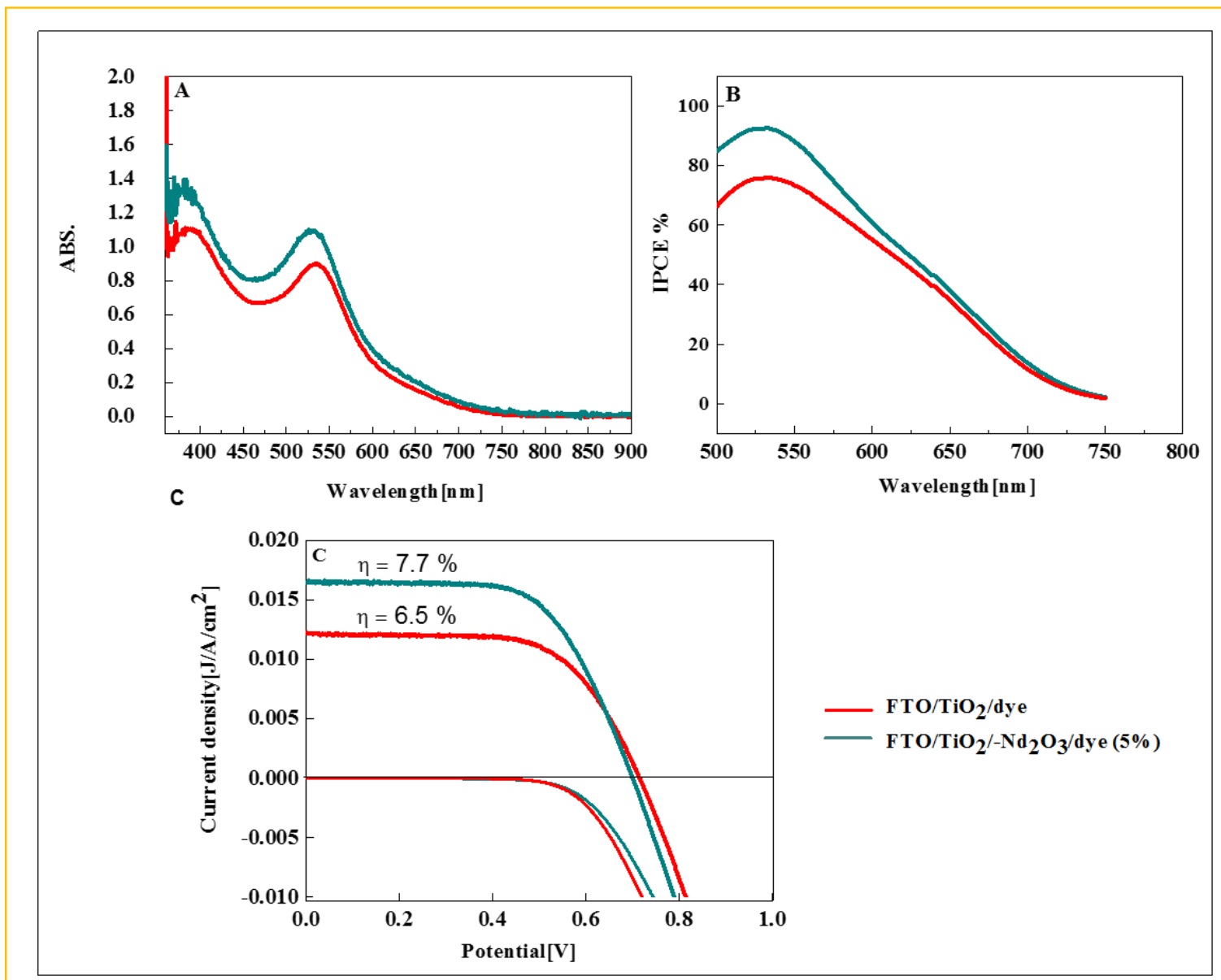

Figure 5.13. UV-vis absorption of FTO/TiO $/$ Dye and $\mathrm{TiO}_{2}-\mathrm{Nd}_{2} \mathrm{O}_{3}(20: 1)$ nano-mesh composite (A). Incident photon-to-current conversion efficiency (B). I-V curve for the same photoanodes used to measure UV-vis and IPCE (C). Dye-soaking time period was $24 \mathrm{~h}$. $\mathrm{TiO}_{2}$ and $\mathrm{Nd}_{2} \mathrm{O}_{3}$ modified paste had double layers made by the doctor blade method. The thickness was $11 \mu \mathrm{m}$.

samples shows greater than that of undoped samples. A property of a solar cell, called the Shockly-Queisser (S-Q) limit, can be determined by IPCE. An intrinsic solar device cannot produce current beyond the $\mathrm{S}-\mathrm{Q}$ limit, however, by modifying its structure (i.e. tandem solar cells) with other materials, the $\mathrm{S}-\mathrm{Q}$ limit might be achievable. For a $\mathrm{TiO}_{2} / \mathrm{N} 719$ dye solar 
cell, the S-Q limit is around $23 \mathrm{~mA} / \mathrm{cm}^{2}$. If the loss in solar intensity is considered, some of the doped devices gave current density beyond the $\mathrm{S}-\mathrm{Q}$ limit. Doping $\mathrm{TiO}_{2}$ by $\mathrm{Nd}_{2} \mathrm{O}_{3}$ may be one of the strategies to beat the S-Q limit in dye-sensitized solar cells.

\subsection{Conclusions}

In conclusion, a dramatically improved efficiency has been observed in a DSSC incorporating a composite working electrode composed of a mixture of $\mathrm{TiO}_{2}$ nanoparticles and $\mathrm{Nd}_{2} \mathrm{O}_{3}$ mesoscale particles. The addition of the large rare earth oxide particles greatly increases the dye uptake of the cell and reduces the charge transfer impedance from the dye into the electrode. It is shown that the incorporation of rare earth oxides into photoanodes is a promising approach to improve the efficiency levels of DSSCs, and through variation in material and particle sizes it is anticipated that further improvements are possible 


\section{CHAPTER 6}

\section{IMPROVED PHOTOVOLTAIC PERFORMANCE OF DYE-SENSITIZED SOLAR CELLS BY USING CHEMICALLY-LINKED GOLD NANOPARTICLES}

Surface modification of $\mathrm{TiO}_{2}$ can bring significant changes on $\mathrm{TiO}_{2}$. In Chapter 3 we prepared stable photoanodes by covalent modification of $\mathrm{TiO}_{2}$ with APTES and N719 dye; PAs did not degrade their PEC performance more than 6 months in air. Chapter 4 showed that surface modification can change the injection dynamics, but the change in injection rate was less than recombination of electrons with electrolyte suggesting that overall device photovoltaics performance should not be affected. In fact, the $\mathrm{V}_{\mathrm{oc}}$, and FF increased with a little decrease in efficiency due to a decrease in current. In these devices, efficiency depends on the amount of dye loaded on $\mathrm{TiO}_{2}$. In Chapter 5, a dramatic increase in photocurrent was observed when commercial $\mathrm{TiO}_{2}$ paste was doped with micro- $\mathrm{Nd}_{2} \mathrm{O}_{3}$. Doped samples showed a higher capacity to load dye on the photoanodes. Experiments showed that the increase in current is not only due to higher amounts of dye that the photoanodes can load, but also due to better charge transport from dye to FTO that $\mathrm{Nd}_{2} \mathrm{O}_{3}$ provided because it can possibly remove traps from $\mathrm{TiO}_{2}$ nanoparticles. A system that can be a tool to improve internal resistance to inhibit recombination but at the same time maintain a smooth flow of charge from dye to $\mathrm{TiO}_{2}, \mathrm{FTO}$, and external circuit is developed in this chapter. Here we describe a photoanode prepared by electrostatic attachment of $4 \mathrm{~nm}$ average diameter $\mathrm{Au}$ nanoparticles (NPs) onto mesoporous $\mathrm{TiO}_{2}$ through a silane linker, 3- 
aminopropyltriethoxysilane (APTES), prior to sensitization with N719 dye. APTES increases resistances to recombination and Au NPs maintain the high photocurrent. The photoanodes were characterized by UV-vis spectroscopy, (ATR-FTIR), (SEM), (AFM), and (EIS) in order to determine the dye-coverage, chemical interaction, and interfacial charge transfer resistances relative to traditionally prepared photoanodes. Ultrafast transient absorption spectroscopy (UTAS) confirms that there is ultrafast interfacial charge injection dynamics $\left(<100 \mathrm{fs}\right.$ ) from excited dye to $\mathrm{TiO}_{2}$ and that a higher percentage of adsorbed dye molecules are involved in the electron injection process, despite an increase in the distance between $\mathrm{N} 719$ dye and the $\mathrm{TiO}_{2}$. Even with the same dye-loading, the Au linked $\mathrm{TiO}_{2}$ dye PA showed the superior PEC performance. The photovoltaic measurements of the AuNPmodified photoanodes show an increase in short circuit current and open circuit voltage. The cell efficiency was $10.4 \%$ compared to $9.0 \%$ for traditional photoanodes. We compared our results to other reports using Au and AgNPs in DSSCs. One major difference in our study is the use of a linker molecule to attach the Au NPs, which improves both the open circuit voltage and short-circuit current.

\subsection{Introduction}

Improving the efficiency and preparing stable devices by a better understanding of the photophysics and photochemistry of materials used in DSSCs are two major goals in DSSC research that may lead to commercialization someday. Since their early report, a great deal of research has been conducted to improve the device performance and understand its mechanisms. $^{30}$ 
The overall sunlight-to-electric-power conversion efficiency (PCE) in percentage $(\eta \%)$ of a DSSC can be expressed mathematically as $\eta \%=\left[\left(\mathrm{J}_{\mathrm{sc}} \times \mathrm{V}_{\text {oc }} \times \mathrm{FF}\right) / \mathrm{P}_{\text {in }}\right] \mathrm{x} 100 \%$, where $\mathrm{J}_{\mathrm{sc}}$ is short-current density, $\mathrm{V}_{\mathrm{oc}}$ is open-circuit photovoltage, FF is the fill factor of the device, and $\mathrm{P}_{\text {in }}$ is the power of incident solar light on the device. ${ }^{22}$ There are several strategies to improve $\eta \%$. One way is to increase $\mathrm{J}_{\mathrm{sc}}$. This can be achieved by maintaining the optical gap (the energy difference between HOMO and LUMO of dye) to $<1.3 \mathrm{eV}$ and retain the optimized charge collecting capacity by harvesting light up to $940 \mathrm{~nm} .{ }^{11}$ Loading a higher amount of active dye on the mesoporous semiconductor film can also increase $\mathbf{J}_{\text {sc. }}$. By increasing the electron injection rate from excited dye to the conduction band of the semiconductor, $\mathrm{J}_{\mathrm{sc}}$ can be increased. ${ }^{201}$ Increasing the $\mathrm{V}_{\mathrm{oc}}$ will also improve $\eta \%$. This can be achieved by increasing the injection rate of excited electrons in the dye, decreasing the recombination of the injected electrons with electrolyte, and moving the Nernstian potential of the electrolyte more positive towards the ground state of the dye. ${ }^{202} \mathrm{FF}$ is another important factor that correlates to the diode quality factor of a silicon $\mathrm{P} / \mathrm{N}$ junction solar cell, which varies from 0 (non-ideal) to 1 (ideal). ${ }^{24}$ The diode quality for DSSCs becomes 1 for devices with perfect suppression of recombination and removal of all trapping states in mesoporous photoanodes. ${ }^{202}$ Several efforts have been made to improve the efficiency of DSSCs by optimizing these parameters.

One method for improving the efficiency of DSSCs is by altering the dye. Examples include molecular engineering of panchromatic dyes to harvest solar light to the near IR region, ${ }^{203}$ modification of dye with alkyl chains to inhibit recombination, ${ }^{67}$ or development of new dyes with a higher extinction coefficient. ${ }^{204}$ Device efficiency can also be improved by creating a stronger interaction between the anchoring groups of dye and $\mathrm{TiO}_{2}$, which can 
increase the electron injection rate. ${ }^{205}$ Semiconductor surface modification is another strategy. Protonation of $\mathrm{TiO}_{2}$ surfaces can increase efficiency because of enhanced dye coupling, a higher injection rate, and lower charge transfer resistance. ${ }^{206}$ Nazeeruddin and coworkers improved device performance by surface modification of both the substrate (FTO) and $\mathrm{TiO}_{2}$ with $\mathrm{TiCl}_{4}$ before and after deposition of bilayers of $20 \mathrm{~nm}$ and $400 \mathrm{~nm}$ $\mathrm{TiO}_{2}$ paste on $\mathrm{FTO}^{27}$ The larger size $\mathrm{TiO}_{2}$ NPs increase light scattering and thus harvest more light, whereas $\mathrm{TiCl}_{4}$ treatment provides well connected bridges among nanoparticles, decreasing the charge transfer resistance. Pre-modification of $\mathrm{FTO}$ by $\mathrm{TiCl}_{4}$ also suppresses recombination from the exposed part of FTO. The formation of uniform, tight, and closepacked electrodeposited $\mathrm{TiO}_{2}$ nanoparticles followed by deposition of $\mathrm{TiO}_{2}$ paste also increases efficiency. ${ }^{207}$ Lao reported an increase in efficiency when $\mathrm{TiO}_{2}$ was pre-modified by 3-aminopropyltriethoxysilane (APTES), ${ }^{99}$ which helps to suppress recombination at the semiconductor-electrolyte junction. Zhang exploited APTES for pre-and post- modification of photoanodes for higher efficiency DSSCs. ${ }^{100}$

An improvement in efficiency has been observed in $\mathrm{TiO}_{2}$ photoanodes by surface modification with metal hydroxides having higher or lower band gaps. ${ }^{23,208}$ Metal hydroxide, such as $\mathrm{Mg}(\mathrm{OH})_{2}$ can create more $\mathrm{OH}$ sites needed for higher dye-loading. Metal oxides, such as $\mathrm{SnO}_{2}, \mathrm{ZnO}$, and $\mathrm{Nb}_{2} \mathrm{O}_{3}$, improve efficiency by suppressing recombination and creating a staircase of energy levels to facilitate the downward flow of excited electrons towards the contact. Quantum dots have recently been employed in DSSCs primarily because of their tunable band gap, high photostability, broad excitation spectra, high extinction coefficient, and multiple exciton generation. ${ }^{209}$ Unfortunately, they show poor orbital coupling with semiconductor oxides such as $\mathrm{TiO}_{2}$ and a low overall power conversion 
efficiency. ${ }^{210}$ Recently, polymer, ${ }^{211}$ aluminum, ${ }^{212}$ and a virus based template ${ }^{213}$ have been used to improve the efficiency of DSSCs by increasing dye-uptake and reducing internal charge transfer resistance by making well-ordered nanoparticles with improved interconnection among them. Different architectures of semiconductors, such as nanowires, nanosheets, opal photonic crystals, and nanotubes, help to remove trap sites, but the efficiency is often low because of low dye coverage. ${ }^{214-216}$

The incorporation of metallic nanostructures into DSSCs has also led to improvements in efficiency of devices. Au and Ag nanostructures have been used as sensitizers ${ }^{217}$ or in $\mathrm{TiO}_{2}-\mathrm{Au}$ or $\mathrm{TiO}_{2}-\mathrm{Ag}$ semiconductor-metal nanocomposites. ${ }^{218,219}$ Because of their plasmonic and conductive properties, Au NPs have an extremely large absorption coefficient compared with usual dye molecules, ${ }^{154}$ the ability to facilitate transfer of excited electrons from dye to semiconductor, ${ }^{220}$ and ability to regenerate dye through plasmons more quickly than from the redox couple. ${ }^{221}$ Au nanoparticles incorporated into $\mathrm{TiO}_{2}$ shift the Fermi level negative compared to the redox couple, increasing the driving force for electrons to move to the external circuit. $^{222} \mathrm{Au}$ has a higher dielectric constant compared to the liquid redox couple in solution, ${ }^{218}$ leading to a reduction in recombination centers by screening the electrons from to $\mathrm{TiO}_{2}{ }^{218}$

Ihara and co-workers in 1997 showed a 149-fold increase in absorption of N719 dye when combined with Ag islands, which paved the way for the use of metal plasmons in DSSC technology. ${ }^{219}$ Standridge and co-workers showed an increase in the extinction coefficient of $\mathrm{N} 3$ dye on $\mathrm{Al}_{2} \mathrm{O}_{3}$-coated $\mathrm{Ag}$ and used an $\mathrm{Ag}-\mathrm{TiO}_{2}$ core to improve the photocurrent in DSSCs in solid state devices. ${ }^{223}$ Wen and co-workers coupled N3 dye with thermally evaporated $\mathrm{Ag}$ islands of $3.3 \mathrm{~nm}$ on $\mathrm{TiO}_{2}$ and reported the absorption of the solar 
spectrum beyond $796 \mathrm{~nm}$ with enhanced photocurrent. ${ }^{224}$ Creating grating surfaces of polycarbonate on FTO and depositing $\mathrm{TiO}_{2}-\mathrm{Au}$ nanocomposites, Baba showed an increase in short circuit current mainly because of the grating-coupled surface plasmon resonance field providing enhancement in the absorption of a porphyrin dye. ${ }^{225}$ Ding and co-workers used nanoimprint lithography to make $\mathrm{TiO}_{2} / \mathrm{Z709} /$ Spiro-OMeTAD/Ag NP solid state dyesensitized solar cells (ss-DSSC) and improved the efficiency from 3.15 to $5.93 \% .226$

Theory suggests that metal plasmons can improve all of the PEC parameters of a DSSC, but a literature survey of up to 30 journals Table 6.1 and 6.2 shows only a few strategies actually are successful to improve overall PEC parameters. Kamat and coworkers showed that plasmon effect enhance photocurrent, but not photovoltage, unless they are coated with an insulator such as $\mathrm{SiO}_{2}{ }^{154}$ Coating $\mathrm{Ag}$ and $\mathrm{Au}$ nanoparticles with $\mathrm{SiO}_{2}$ also improves the device stability in the $\mathrm{I}^{-} / \mathrm{I}_{3}{ }^{-}$electrolyte as described by Standridge and Brown. ${ }^{227,218}$ Kamat and coworkers mixed 5-6 nm octylamine-capped Au nanoparticles with $\mathrm{TiO}_{2}$ in a 7:1000 ratio and heated them to $500{ }^{\circ} \mathrm{C}$ before dye-loading, improving the efficiency from 9.37 to 10.21\%. Wang and co-workers obtained $10.8 \%$ efficient DSSCs by mixing $5 \mathrm{~nm} \mathrm{Au}$ nanoparticles with $\mathrm{TiO}_{2}$ in a 2:1000 ratio, which was a $13 \%$ improvement. This is the maximum efficiency reported so far using metal plasmons in DSSCs. ${ }^{228}$ Recently, Gangishetty and co-workers improved the efficiency from 5.6 to $7.4 \%$ by using $\mathrm{SiO}_{2}$-coated triangular Ag nanoplates, which can absorb near-IR solar light when incorporated with dye. ${ }^{227}$ DSSCs with plasmonic nanostructures usually show improvements in photocurrent and overall efficiency, but not usually $\mathrm{V}_{\mathrm{oc}}$ and FF. There is therefore still room for improvement in the use of plasmonic nanostructures for solar cell applications. 
In this work, we immobilize $4 \mathrm{~nm}$ diameter $\mathrm{Au}$ NPs onto mesoporous $\mathrm{TiO}_{2}$ using a molecular linker, 3-aminopropyltriethoxysilane (APTES), and adsorb N719 dye onto the

Table 6.1. General survey of literature for the study of PEC parameters of a DSSC.

\begin{tabular}{|c|c|c|c|c|c|c|c|}
\hline SN & $\begin{array}{c}\mathrm{I}_{\mathrm{ph}} \\
\left(\mathrm{mA} / \mathrm{cm}^{2}\right)\end{array}$ & $\begin{array}{l}V_{o c} \\
(V)\end{array}$ & $\mathbf{F F}$ & $\eta \%$ & Reference & Note & $\begin{array}{c}\uparrow \text { in all PEC } \\
\text { para. }\end{array}$ \\
\hline 1 & $11.83 \uparrow$ & $0.71 \downarrow$ & $0.63 \downarrow$ & $6.26 \uparrow$ & Guo, $\mathrm{K}^{1}$ & $\mathrm{TiO}_{2} / \mathrm{Ag}$ & \\
\hline 2 & $5.82 \uparrow$ & $0.62 \downarrow$ & $0.62 \sim$ & $2.24 \uparrow$ & $\mathrm{Li}, \mathrm{H}^{3}{ }^{3}$ & $\mathrm{ZnO} / \mathrm{Au}$ & \\
\hline 3 & $12.7 \uparrow$ & $\begin{array}{c}0.714 \\
\uparrow\end{array}$ & $0.63 \downarrow$ & $5.59 \uparrow$ & Jiang, $W .5$ & $\mathrm{TiO}_{2} / \mathrm{Ag}$ & \\
\hline 4 & $3.37 \uparrow$ & $\begin{array}{c}0.651 \\
\downarrow\end{array}$ & $0.62 \downarrow$ & $1.35 \uparrow$ & Wu,K-J. ${ }^{7}$ & $\mathrm{HCl}$ treat & \\
\hline 5 & $16.75 \uparrow$ & $\begin{array}{c}0.846 \\
\uparrow\end{array}$ & $0.72 \downarrow$ & $10.25 \uparrow$ & Dang, $X{ }^{9}$ & $\mathrm{TiO}_{2} / \mathrm{Ag} / \mathrm{Au}$ & \\
\hline 6 & $11.59 \uparrow$ & 0.80 & 0.56 & $6.40 \uparrow$ & Sheehan,W. ${ }^{11}$ & $\mathrm{TiO}_{2} / \mathrm{Au}$ & \\
\hline 7 & $20.21 \uparrow$ & $0.73 \downarrow$ & $0.69 \downarrow$ & $10.21 \uparrow$ & Choi, H. ${ }^{13}$ & $\mathrm{TiO}_{2} / \mathrm{Au}$ & \\
\hline 8 & $13.20 \uparrow$ & $\begin{array}{c}0.74 \\
\uparrow\end{array}$ & $0.61 \uparrow$ & $6.00 \uparrow$ & Muduli, S. ${ }^{15}$ & $\mathrm{TiO}_{2} / \mathrm{Au}$ & $\sqrt{ }$ \\
\hline 9 & $16.50 \uparrow$ & $0.82 \uparrow$ & $0.67 \sim$ & $9.00 \uparrow$ & Qi, J. (Ag NP) ${ }^{17}$ & $\mathrm{TiO}_{2} / \mathrm{Au}$ & \\
\hline 10 & $10.44 \uparrow$ & $0.82 \uparrow$ & $0.69 \uparrow$ & $5.93 \uparrow$ & Ding, I-K. (Ag NP) $)^{19}$ & $\mathrm{TiO}_{2} / \mathrm{Au}$ & $\sqrt{ }$ \\
\hline 11 & $3.37 \uparrow$ & $0.76 \uparrow$ & $0.76 \uparrow$ & $1.95 \uparrow$ & Brown, M.D. ${ }^{21}$ & $\mathrm{TiO}_{2} / \mathrm{Au}$ & $\sqrt{ }$ \\
\hline 12 & $6.18 \uparrow$ & $0.70 \uparrow$ & $0.40 \uparrow$ & $1.57 \uparrow$ & Chou, C.S. ${ }^{22}$ & $\mathrm{TiO}_{2} / \mathrm{Au}$ & $\sqrt{ }$ \\
\hline 13 & $19.51 \uparrow$ & $0.71 \downarrow$ & $0.56 \downarrow$ & $7.85 \uparrow$ & $X u, Q .{ }^{23}$ & $\mathrm{TiO}_{2} / \mathrm{Au}$ & \\
\hline 14 & $12.62 \uparrow$ & $\begin{array}{c}0.785 \\
\downarrow\end{array}$ & $0.71 \downarrow$ & $7.04 \uparrow$ & Ramasamy,P. ${ }^{24}$ & $\mathrm{TiO}_{2} / \mathrm{Au}$ & \\
\hline 15 & $15.71 \uparrow$ & NA & NA & $10.10 \uparrow$ & $\mathrm{Li}, \mathrm{Y} .25$ & $\mathrm{TiO}_{2} / \mathrm{Au}$ & \\
\hline 16 & $13.72 \uparrow$ & $0.83 \uparrow$ & $0.74 \uparrow$ & $8.46 \uparrow$ & Chen, P-Y. ${ }^{26}$ & $\mathrm{TiO}_{2} / \mathrm{Au}$ & \\
\hline 17 & $19.40 \uparrow$ & $0.80 \uparrow$ & $0.70 \sim$ & $10.80 \uparrow$ & Wang, Q. ${ }^{27}$ & $\mathrm{TiO}_{2} / \mathrm{Au}$ & \\
\hline 18 & $13.71 \uparrow$ & $0.75 \uparrow$ & $0.61 \uparrow$ & $7.65 \uparrow$ & Chang, $S .{ }^{28}$ & $\mathrm{TiO}_{2} / \mathrm{Au}$ & $\sqrt{ }$ \\
\hline 19 & $11.76 \uparrow$ & $0.71 \sim$ & $0.66 \sim$ & $5.55 \uparrow$ & $\mathrm{Li}, \mathrm{H} .\left(\mathrm{AuSiO}_{2}\right)^{29}$ & $\mathrm{TiO}_{2} / \mathrm{Au}$ & \\
\hline 20 & $15.1 \uparrow$ & $0.77 \uparrow$ & $0.67 \uparrow$ & $7.77 \uparrow$ & Naphade, R. A. ${ }^{30}$ & $\mathrm{TiO}_{2} / \mathrm{Au}$ & $\sqrt{ }$ \\
\hline
\end{tabular}

$\mathrm{TiO}_{2} / \mathrm{APTES} / \mathrm{Au}$ NPs film. This strategy is very different compared to almost all other strategies, which incorporate the metal nanostructures into the $\mathrm{TiO}_{2}$ paste before preparation and calcination and then adsorb dye to the $\mathrm{TiO}_{2}$-metal nanostructures composite. Our 
semiconductor material is not a mixed nanocomposite. It is instead a layered $\mathrm{TiO}_{2} / \mathrm{APTES} / \mathrm{Au}$ NPs material. With nanocomposites, the sintering prior to dye-loading

Table 6.2. General survey of literature for the study of PEC parameters of a DSSC continue from table one. These are reported for low efficiency.

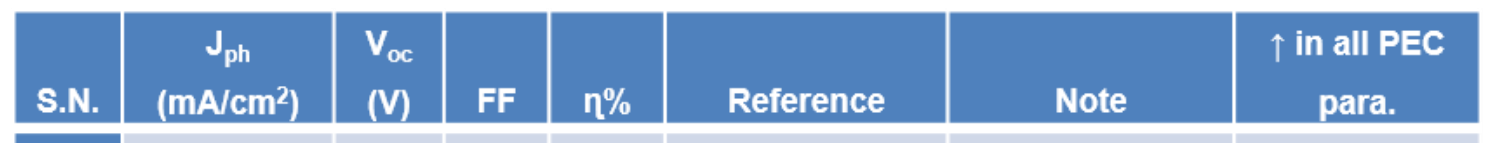

\begin{tabular}{|c|c|c|c|c|c|c|c|}
\hline 1 & $\uparrow$ & $\uparrow$ & $\downarrow$ & $\uparrow$ & Chang, S. ${ }^{2}$ & $\mathrm{TiO}_{2} / \mathrm{Au}<3 \%$ & \\
\hline 2 & $\uparrow$ & $\uparrow$ & $\downarrow$ & $\uparrow$ & Lin, S. ${ }^{4}$ & $\mathrm{TiO}_{2} / \mathrm{Au}<3 \%$ & \\
\hline 3 & $\uparrow$ & $\sim$ & $\sim$ & $\uparrow$ & Nahm, C. ${ }^{6}$ & $\mathrm{TiO}_{2} / \mathrm{Au}<3 \%$ & \\
\hline 4 & $\uparrow$ & $\sim$ & $\downarrow$ & $\uparrow$ & Hou, W. ${ }^{8}$ & $\mathrm{TiO}_{2} / \mathrm{Au}$ & \\
\hline 5 & $\uparrow$ & $\uparrow$ & $\uparrow$ & $\uparrow$ & Bora, T. ${ }^{10}$ & $\mathrm{ZnO} / \mathrm{Au}$ & $\sqrt{ }$ \\
\hline 6 & $\uparrow$ & $\uparrow$ & $\uparrow$ & $\uparrow$ & Sarkar, S. ${ }^{12}$ & $\mathrm{ZnO} / \mathrm{Au}$ & $\sqrt{ }$ \\
\hline 7 & $\uparrow$ & $\sim$ & $\downarrow$ & $\uparrow$ & Ihara, M. ${ }^{14}$ & $\mathrm{TiO}_{2} / \mathrm{Au}$ & \\
\hline 8 & $\uparrow$ & $\downarrow$ & $\sim$ & $\uparrow$ & Wen, C. ${ }^{16}$ & $\mathrm{TiO}_{2} / \mathrm{Au}$ & \\
\hline 9 & $\uparrow$ & $\downarrow$ & $\uparrow$ & $\uparrow$ & Gaffaari, M. ${ }^{18}$ & & \\
\hline 10 & $\downarrow$ & $\downarrow$ & $\uparrow$ & $\downarrow$ & Peh, C.K.N. ${ }^{20}$ & & \\
\hline
\end{tabular}

removes all of the organic material used to stabilize the metal NPs. While the molecular linker may decrease the rate of electron injection, Au NPs facilitate electron transfer. ${ }^{229}$ Also, the presence of the molecular linker can reduce recombination to increase $\mathrm{V}_{\mathrm{oc}}$. Importantly, the N719 dye interacts well with the high surface area Au NPs, providing large dye loading. ${ }^{230}$ The synthesis and deposition of $\mathrm{Au}$ NPs on $\mathrm{TiO}_{2}$ in this manner is a simple two-step process. The use of molecular linkers for incorporating metallic plasmonic nanostructures into DSSCs is a promising avenue to improve efficiency and has significant potential for commercialization. Study of this novel DSSC also helps us to better our understanding of the photovoltaic processes. 


\subsection{Experimental Details}

Preparation of $\mathrm{TiO}_{2}$ Film. Dyesol $\mathrm{TiO}_{2}$ paste was used as available without further treatment. FTO slides were cleaned by sonication in acetone, ethanol and IPA for $10 \mathrm{~min}$ each, and at the end rinsed with IPA, and dried under a stream of $\mathrm{N}_{2}$ gas. The paste was deposited on the FTO slide by screen printing or the doctor blade method. Prior to $\mathrm{TiO}_{2}$ deposition, $\mathrm{FTO}$ slides were treated with $\mathrm{TiCl}_{4}$ by soaking in $40 \mathrm{mM} \mathrm{TiCl}_{4}$ solution at $70{ }^{\circ} \mathrm{C}$ for 30 min. ${ }^{27}$ A $1.0 \mathrm{~cm}^{2}$ area was made on the $90 \mathrm{~T}$ screen printer and $\mathrm{TiO}_{2}$ was deposited on the FTO slide three times to obtain a thickness of $8.75 \pm 0.85 \mu \mathrm{m}$. The deposited paste was first heated at $125^{\circ} \mathrm{C}$ for at least 6 min to level the viscous paste on FTO and then sintered at $500{ }^{\circ} \mathrm{C}$ for $1 \mathrm{~h}$ in a $1300 \mathrm{BL}$ Barnstead Thermolyne Furnace. The sintered $\mathrm{TiO}_{2}$ film was post-treated with $\mathrm{TiCl}_{4}$ in the same fashion and sintered again at $500{ }^{\circ} \mathrm{C}$ for $1 \mathrm{~h}$. The same procedure was adopted for the doctor blade method, except that the $\mathrm{TiO}_{2}$ paste was exposed to ethanol vapor before leveling at $125{ }^{\circ} \mathrm{C}$. To get the optimized photoelectrochemical (PEC) parameters, an extra scattering layer was deposited on the $\mathrm{TiO}_{2}$ paste, exposed to ethanol for $1 \mathrm{~min}$, heated at $125{ }^{\circ} \mathrm{C}$ to level the paste, sintered at $500{ }^{\circ} \mathrm{C}$, and treated with $\mathrm{TiCl}_{4}$ again. Finally, different areas $\left(0.30 \mathrm{~cm}^{2}, 0.25 \mathrm{~cm}^{2}\right.$ and $\left.0.16 \mathrm{~cm}^{2}\right)$ of $\mathrm{TiO}_{2}$ paste were made by gently removing extra $\mathrm{TiO}_{2}$ with a sharp blade. This electrode is referred to as an $\mathrm{FTO} / \mathrm{TiO}_{2}$ electrode.

$\mathrm{TiO}_{2}$ Functionalization with APTES linker. The $\mathrm{FTO} / \mathrm{TiO}_{2}$ electrode was placed inside a solution containing $10 \mathrm{~mL}$ of IPA, $100 \mu \mathrm{L}$ of APTES, and 3-4 drops of nanopure water. ${ }^{108}$ The solution was placed in a water bath at $70{ }^{\circ} \mathrm{C}$ for $30 \mathrm{~min}$. The electrode was rinsed thoroughly with IPA and dried under a $\mathrm{N}_{2}$ stream for $1 \mathrm{~min}$. This is referred to as an FTO/TiO $/$ APTES electrode. 
Gold nanoparticles (Au NPs) Modification. Au NPs of $4 \mathrm{~nm}$ average diameter were synthesized as described in literature. ${ }^{231}$ Briefly, $147 \mu \mathrm{L}$ of $1 \%$ sodium citrate solution was added in $20 \mathrm{~mL}$ of vigorously stirred $2.5 \times 10^{-4} \mathrm{M} \mathrm{AuCl}_{4}^{-}$solution. Next, $0.6 \mathrm{~mL}$ of ice-cold aqueous $0.1 \mathrm{M}$ sodium borohydride was added at once. The slightly yellowish solution turned into a red wine color. The Au NP solution was stirred at least $2 \mathrm{~h}$ continuously. The $\mathrm{FTO} / \mathrm{TiO}_{2} / \mathrm{APTES}$ electrodes were soaked in the Au NP solution for 15 min to attach $\mathrm{Au}$ NPs electrostatically. ${ }^{98}$ This is referred to as an $\mathrm{FTO} / \mathrm{TiO}_{2} / \mathrm{APTES} / \mathrm{AuNPs}$ electrode.

Dye Sensitization. Sensitization of the $\mathrm{FTO} / \mathrm{TiO}_{2}, \mathrm{FTO} / \mathrm{TiO}_{2} / \mathrm{APTES}$, and FTO/TiO $/$ APTES/AuNPs electrodes was achieved by immersing the electrodes into a 0.3 mM solution of N719 dye in a 1:1 mixture of acetonitrile and $t$-butanol for appropriate amount of time to load the same amount of dye on $\mathrm{TiO}_{2}$. These are referred to as

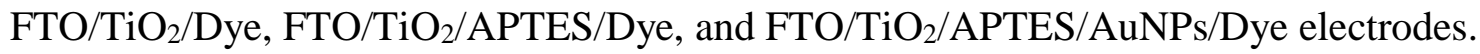
To get optimized values of the PEC parameters, $\mathrm{FTO} / \mathrm{TiO}_{2}$ and $\mathrm{FTO} / \mathrm{TiO}_{2} / \mathrm{APTES} / \mathrm{AuNPs}$ electrodes were soaked in dye solution for $24 \mathrm{~h}$. To get similar coverages, the PAs were soaked in dye for various times as described in the main text.

PEC Measurements. The N719 dye-sensitized photoanodes acted as the working electrode. A counter electrode was produced by coating a cleaned FTO slide with a drop of Platisol and heating to $500{ }^{\circ} \mathrm{C}$ for $20 \mathrm{~min}$. Both the working electrode and counter electrode were silver painted on the edges of the conductive sides to produce good electrical contact. The PEC parameters were measured in sandwiched cells prepared by sealing with Surlyn (plastic ionomer) inserted between the working electrode and counter electrode and heating at $125{ }^{\circ} \mathrm{C}$ for a minutes by placing in a thermal press. Liquid electrolyte was filled into the mesoporous films by vacuum back filling and the hole was first sealed by liquid sealant and 
second by liquid Crystalbond adhesive wax bought from Ted Pella Inc., USA. When surlyn sealant is placed on top of the hole, a hot (around $240^{\circ} \mathrm{C}$ ) soldering rod is gently placed on the sealant, the sealant melts and fills the hole. Good electrical contact was made by soldering indium chunk metal on exposed parts of the conductive sides of PA and CE. The cells were illuminated with simulated 1.5 AM solar light (Newport Oriel, USA). The power density was maintained at $100 \mathrm{~mW} / \mathrm{cm}^{2}$. Current-voltage curves were obtained by applying an external bias to the cells and measuring the current with a Model 273A EG\&G Princeton Applied Research Potentiostat. The intensity of the light was set based on the voltage response of a commercially purchased, calibrated $1 \mathrm{~cm}^{2}$ reference silicon solar cell from Abet Technologies, Inc., USA with in-built KG5 filter (uncertainty $=8 \%$ ). The light source was set to 1 Sun by adjusting the light intensity until the reference silicon solar cell gave a reading of $100 \mathrm{mV}$ as measured by a voltmeter. A homemade optical sample holder was used to mount the reference silicon solar cell. After setting the light intensity, the reference silicon solar cell was replaced with the fabricated dye-sensitized solar cell (DSSC) under study in the same location at the same distance from the light source as dictated by the optical holder. The devices were masked by sticky black paper tape with an open window area of $0.16 \mathrm{~cm}^{2}$ prepared by a laser printer. Black electrical tape was used to seal all edges of the DSSC devices in order to prevent light piping.

Spectroscopic Characterization. Absorption spectra of the photoanodes were measured with a Varian Cary 50 Win UV-vis spectrophotometer (Australia). ATR-FTIR (Perkin Elmer, Spectrum series 100) spectroscopy measurements were performed before and after dye attachment to confirm the surface chemistry. The photoanodes were placed upside down onto the ZnSe crystal in the ATR-FTIR spectrometer and held at a pressure of 
80 psi. The spectrometer was scanned from 500 to $4000 \mathrm{~cm}^{-1}$ at a resolution of $4 \mathrm{~cm}^{-1}$. Electrochemical impedance spectroscopy measurements were performed using a CHI 660a electrochemical workstation. A $10 \mathrm{mV}$ AC perturbation at open circuit voltage was applied with a frequency range of $100 \mathrm{kHz}$ to $10 \mathrm{MHz}$. The photoanodes were maintained at the open circuit voltage by being illuminated with white light from an optical fiber-coupled radiation source (Fiber Optic Illuminator Model 190, Dolan-Jenner, USA). The DSSCs were connected in a two-terminal configuration. The photoanode was connected as the working electrode and the platinum counter electrode was connected to the reference and counter electrode.

\subsection{Results}

Film Preparation and Imaging. We assembled three different types of photoanodes as described in the experimental section, namely $\mathrm{FTO} / \mathrm{TiO}_{2} / \mathrm{Dye}_{\mathrm{FTO}} / \mathrm{TiO}_{2} / \mathrm{APTES} / \mathrm{Dye}$, and FTO/TiO 2 /APTES/AuNPs/Dye. A detailed scheme of the three photoanodes is shown in Scheme 6.1 and Scheme 6.2, which also shows the different dye bonding interactions. In conventional $\mathrm{FTO} / \mathrm{TiO}_{2} /$ Dye photoanodes, dye attaches directly to $\mathrm{TiO}_{2}$ by bidentate bridging to $\mathrm{Ti}$ or through $\mathrm{OH}$ sites via hydrogen bonding. ${ }^{70}$ In the $\mathrm{FTO} / \mathrm{TiO}_{2} / \mathrm{APTES}$ photoanode, dye can be attach directly to $\mathrm{TiO}_{2}$ (Ti or $\mathrm{OH}$ sites), or to the $\mathrm{NH}_{2} / \mathrm{NH}_{3}{ }^{+}$sites of APTES (electrostatically or by H-bonding). N719 dye may bind to Au NP modified photoanodes through the thiocyanate by an Au-S bond. ${ }^{230}$ 
Scheme 6.1. Illustration of potential dye location on $\mathrm{FTO} / \mathrm{TiO}_{2}, \mathrm{FTO} / \mathrm{TiO}_{2} / \mathrm{APTES}$, and FTO/TiO ${ }_{2} /$ APTES/Au NPs.

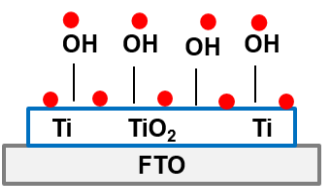

$\mathrm{FTO}^{\mathrm{TiO}}{ }_{2} /$ Dye
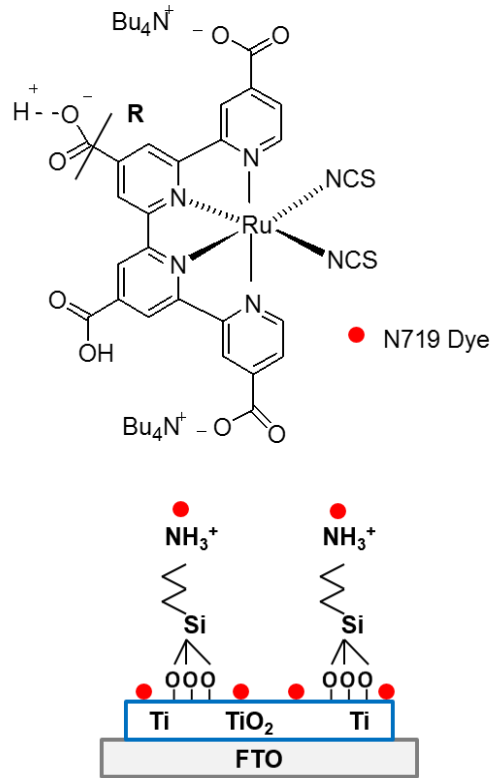

FTO/TiO $/$ /APTES/Dye

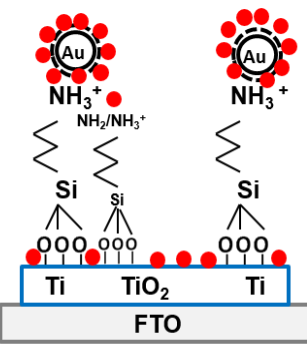

FTO/TiO $2 /$ APTES/AuNP/Dye

Scheme 6.2. Illustration of potential interactions of dye in $\mathrm{FTO} / \mathrm{TiO}_{2}, \mathrm{FTO} / \mathrm{TiO}_{2} / \mathrm{APTES}$, and FTO/TiO $/$ /APTES/Au NPs.

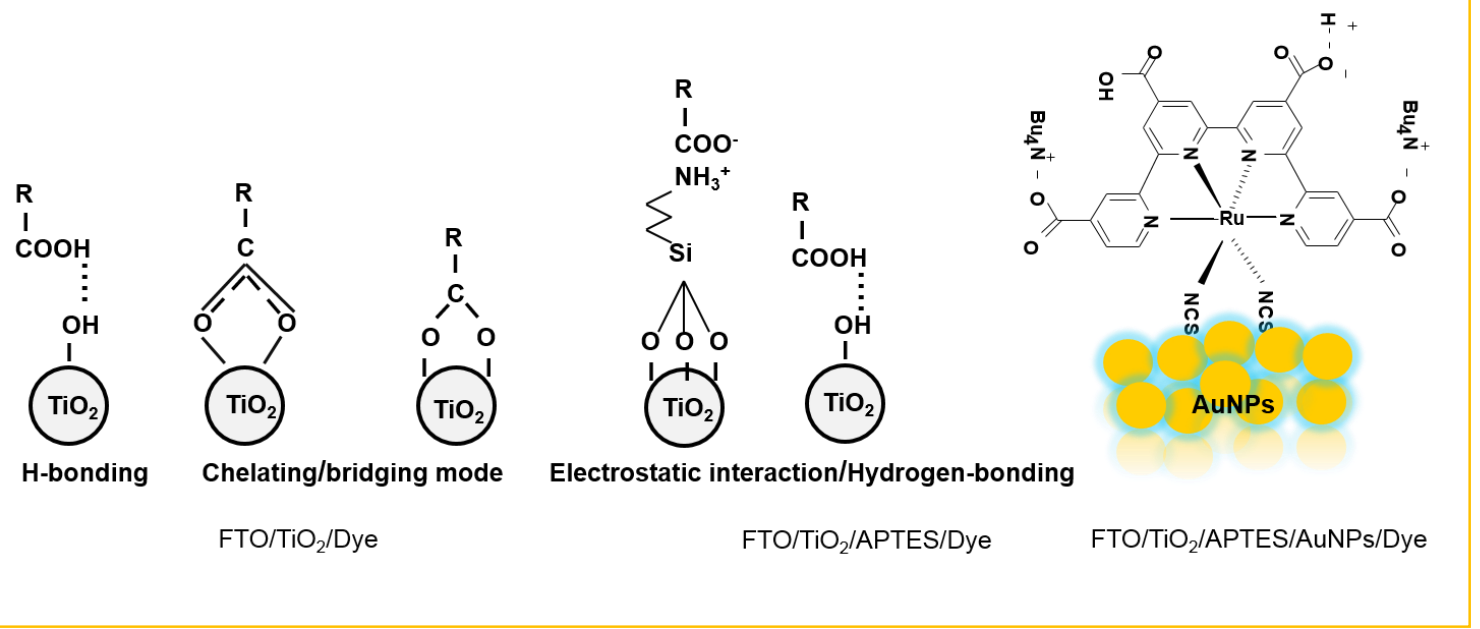


Figure 6.1 shows SEM images of $\mathrm{FTO} / \mathrm{TiO}_{2}($ Frame A) and FTO/TiO $2 / \mathrm{APTES} / \mathrm{AuNPs}$ (Frame B) of electrodes to be used as photoanodes. The darker grey mesoporous network of 18-50 nm diameter nanoparticles is the $\mathrm{TiO}_{2}$ film as shown in Frame A. Figure 6.1B also shows this same mesoporous network, but with brighter spots on top of it, which represent
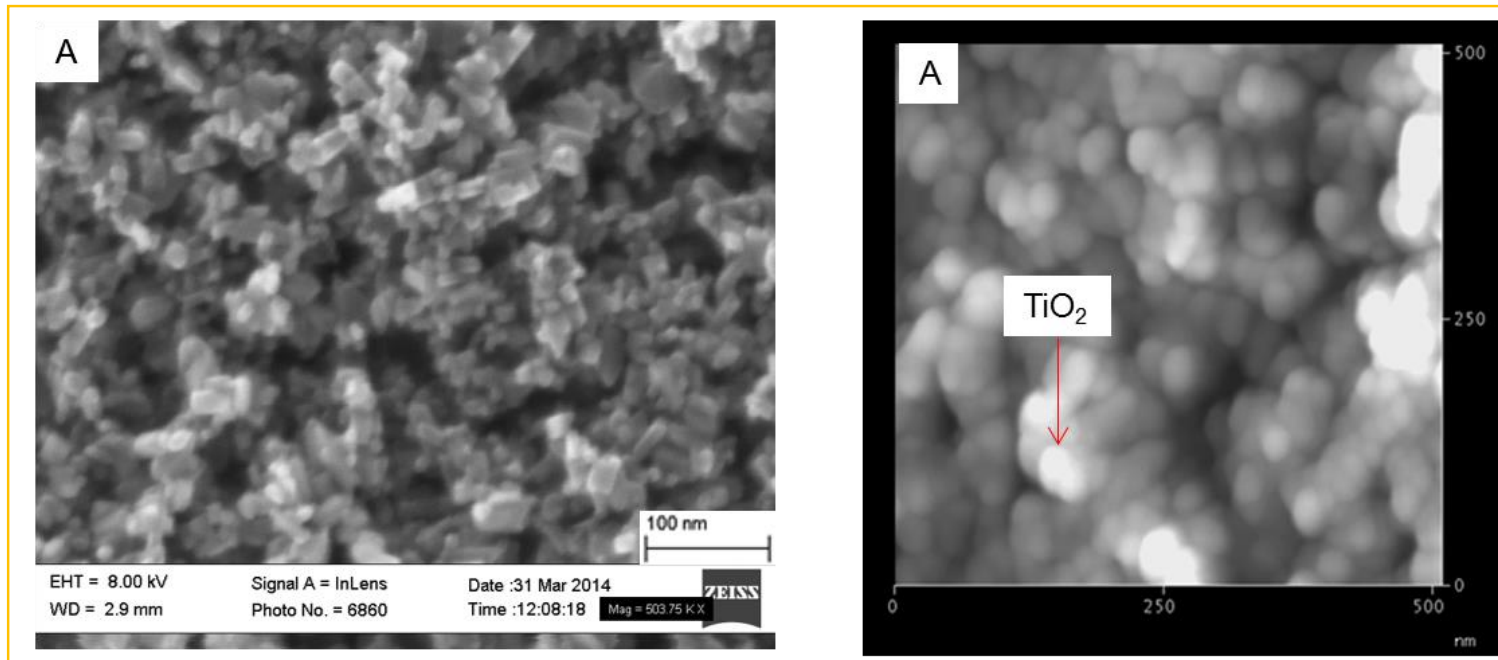

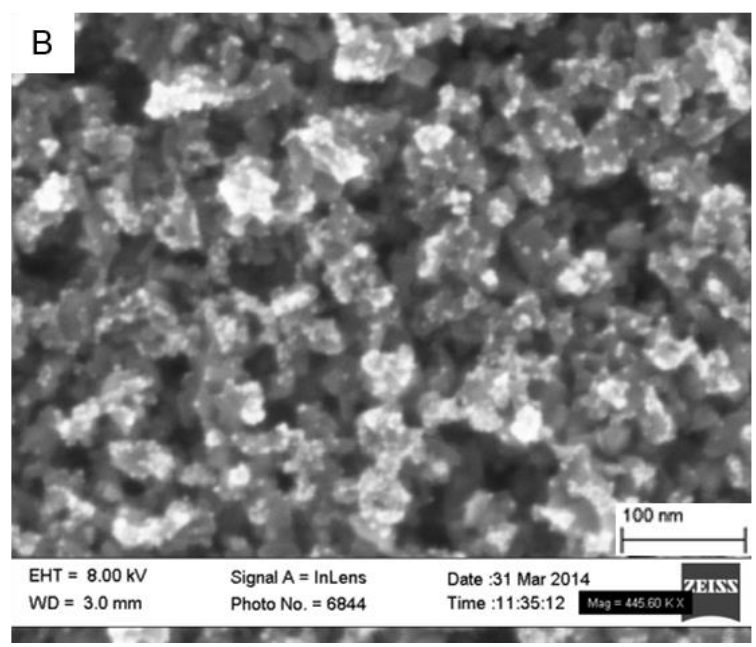

Figure 6.1. SEM images of (A) $\mathrm{TiO}_{2}$ and (B) $\mathrm{TiO}_{2} / \mathrm{APTES} / \mathrm{AuNPs}$

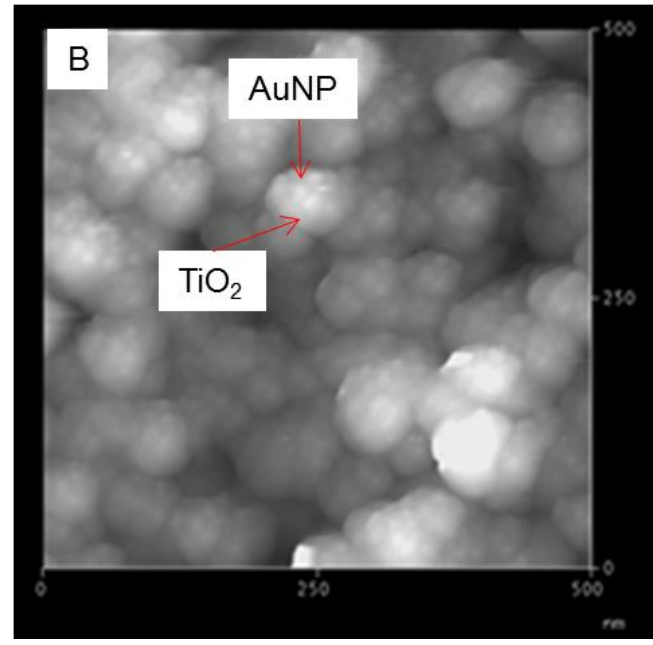

Figure 6.2. AFM images of (A) $\mathrm{TiO}_{2}$ and (B) $\mathrm{TiO}_{2} / \mathrm{APTES} / \mathrm{AuNPs}$

the smaller sized ( 3-6 nm diameter) $\mathrm{Au}$ NPs on top of the $\mathrm{TiO}_{2}$. Larger sizes of $\mathrm{TiO}_{2}$ or $\mathrm{Au}$ NPs are due to aggregates. Figure 6.2 shows AFM images of $\mathrm{FTO} / \mathrm{TiO}_{2}($ Frame A) and FTO/TiO $2 /$ APTES/AuNPs (Frame B) photoanodes. The AFM image of $\mathrm{FTO} / \mathrm{TiO}_{2}$ shows 
the nanostructure of the thin film. The $\mathrm{FTO} / \mathrm{TiO}_{2} / \mathrm{APTES} / \mathrm{AuNPs}$ surface looks similar but also had small nanoscale bumps on the surface attributed to the Au NPs. This reveals fairly a large number of coverage of Au NPs on the surface. The image of the FTO/TIO 2 /APTES/AuNPs, which we attribute to the $\sim 4 \mathrm{~nm}$ diameter Au NPs attached to the surface through APTES. Although it is hard to quantify, there appears to be a substantial coverage of $\mathrm{Au}$ NPs on the $\mathrm{TiO}_{2}$ surface. The SEM and AFM images confirm attachment of the Au NPs and give a general idea of the coverage.

Spectroscopic Characterization. Figure 6.3 shows ATR-FTIR spectra of the three different photoanodes before sensitization with $\mathrm{N} 719$ dye. The FTO/TiO 2 electrode has two peaks around $3300-3600 \mathrm{~cm}^{-1}$ and $1630 \mathrm{~cm}^{-1}$, due to stretching and bending modes of water

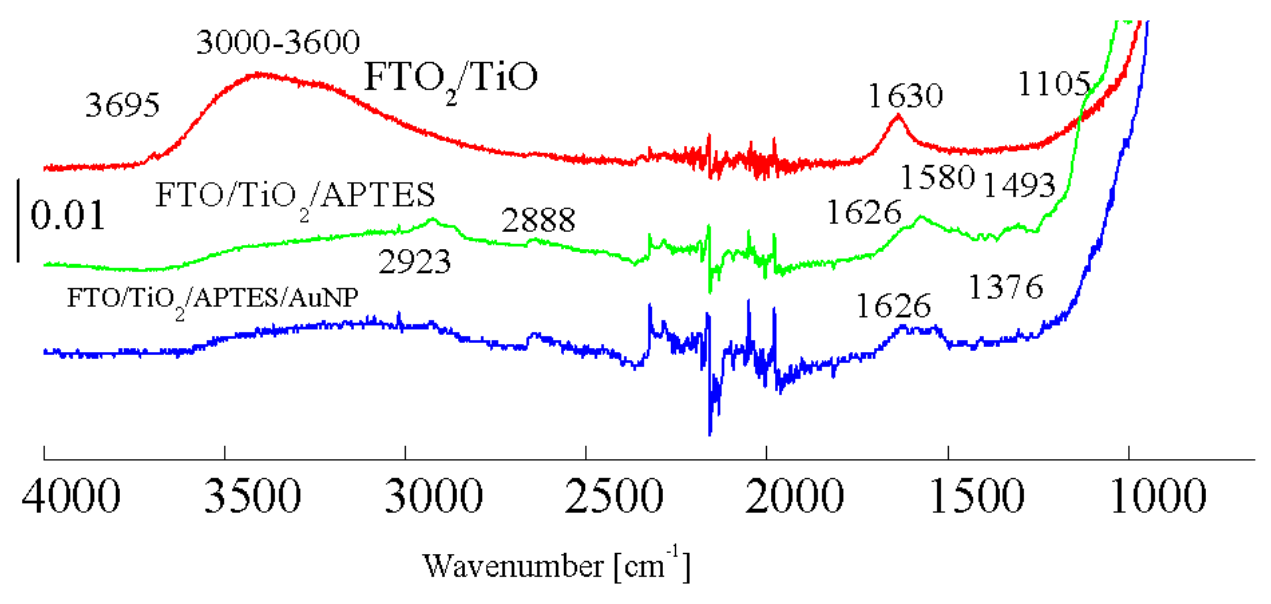

Figure 6.3. ATR-FTIR spectroscopy of $\mathrm{FTO} / \mathrm{TiO}_{2}$ (red), $\mathrm{FTO} / \mathrm{TiO}_{2} / \mathrm{APTES}$ (green), and FTO/TiO 2 /APTES/AuNPs (blue).

molecules adsorbed on $\mathrm{TiO}_{2}$, respectively. ${ }^{232}$ After chemical modification of $\mathrm{TiO}_{2}$ with APTES, new peaks at $2923 \mathrm{~cm}^{-1}\left(\mathrm{CH}_{2}\right.$, sym $)$ and $2888 \mathrm{~cm}^{-1}\left(\mathrm{CH}_{2}\right.$, asym $)$ appeared in addition to that at $1580 \mathrm{~cm}^{-1}$ (deformed $\mathrm{NH}_{3}{ }^{+}$). A peak at $1105 \mathrm{~cm}^{-1}$ attributed to $\mathrm{Si}-\mathrm{O}-\mathrm{Si}$ bonds also appeared. The peak at $1580 \mathrm{~cm}^{-1}$ confirms the binding of APTES and formation of free amine layer on $\mathrm{TiO}_{2}{ }^{100}$ Following electrostatic attachment of citrate-capped, negatively- 
charged gold nanoparticles to the $\mathrm{NH}_{2} / \mathrm{NH}_{3}{ }^{+}$groups, the spectrum looked very similar, making it difficult to confirm Au NPs attachment by FTIR.

Figure 6.4 also shows the ATR-FIR spectra of the three photoanodes after dye sensitization. In all IR spectra, the peaks at 1604 and $1375 \mathrm{~cm}^{-1}$ correspond to the asymmetric and symmetric stretch of the carboxylate group of dye, respectively. The peak at $1542 \mathrm{~cm}^{-1}$ corresponds to the bipyridyl group and the peak at $2109 \mathrm{~cm}^{-1}$ is due to the $\mathrm{CN}$ stretch from the two SCN ligands on each N719 dye molecule. These peaks confirm dye

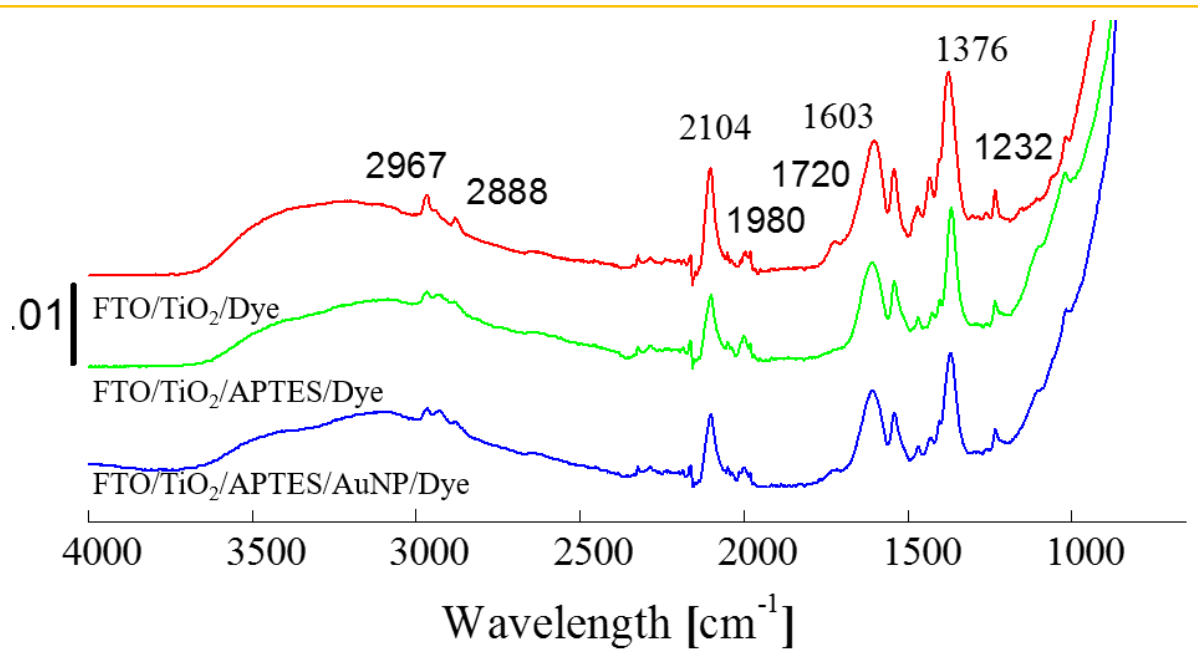

Figure 6.4. ATR-FTIR spectroscopy of conventional FTO/TiO $2 /$ Dye (red) $\mathrm{FTO} / \mathrm{TiO}_{2} / \mathrm{APTES}$ /Dye (green) and FTO/TiO $/$ /APTES/AuNPs/Dye (blue).

attachment to the PAs in all cases. The peak at $1720 \mathrm{~cm}^{-1}$ is due to protonated $\mathrm{COOH}$ groups on dye, showing that not all of the groups are bound as carboxylates. The free $\mathrm{COOH}$ peak is more prevalent in $\mathrm{FTO} / \mathrm{TiO}_{2} / \mathrm{Dye}$ and $\mathrm{FTO} / \mathrm{TiO}_{2} / \mathrm{APTES} / \mathrm{AuNPs} / \mathrm{Dye}$ photoanodes. The dye peaks are very similar in all PAs actually, suggesting that the presence of APTES and Au NPs does not change the chemical environment of the dye significantly.

Figure 6.5 compares the UV-vis spectrum and I-V curves of DSSCs with $\mathrm{FTO} / \mathrm{TiO}_{2} /$ Dye and $\mathrm{FTO} / \mathrm{TiO}_{2} / \mathrm{APTES} / \mathrm{AuNPs} /$ Dye PAs that were prepared with a screenprinted film of $18 \mathrm{~nm} \mathrm{TiO}_{2} \mathrm{NPs}$ paste and no scattering layer to allow UV-vis spectroscopy 
measurements. They were soaked in dye solution for $24 \mathrm{~h}$ and in pure solvent for $3 \mathrm{~h}$ to
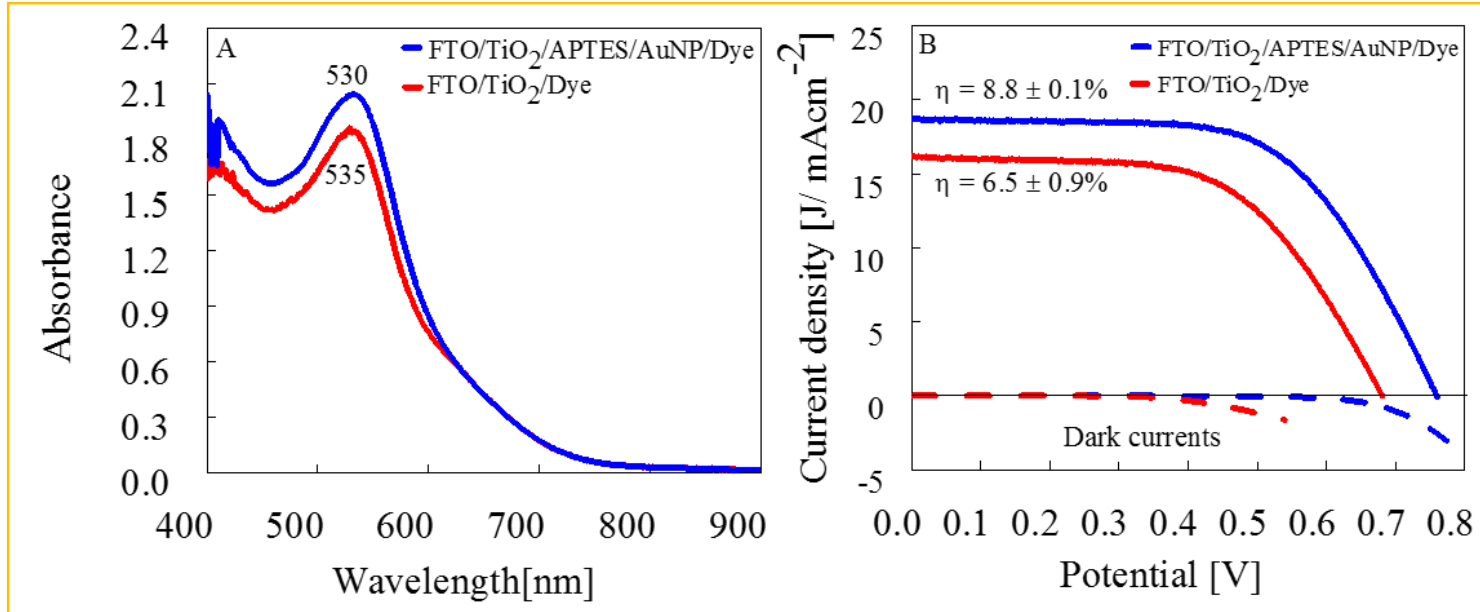

Figure 6.5. UV-Vis spectra (A) and $\mathrm{I}-\mathrm{V}$ curves (B) of $\mathrm{FTO} / \mathrm{TiO}_{2} / \mathrm{Dye}$ (red) and $\mathrm{FTO} / \mathrm{TiO}_{2} / \mathrm{APTES} / \mathrm{AuNP}$ /Dye (blue) photoanodes. The $\mathrm{TiO}_{2}$ paste was made from DSL $18 \mathrm{NR}-$ $\mathrm{T}$ by screen printing, the area was $0.25 \mathrm{~cm}^{2}$, and there was no scattering layer to allow UV-vis analysis. The efficiency of the DSSC modified with Au NPs showed a 35\% increase in efficiency by increasing both photocurrent and photovoltage.

Table 6.3. PEC parameters for screen printed PAs.

\begin{tabular}{|c|c|c|c|c|}
\hline \multirow[b]{2}{*}{ S.N. } & \multicolumn{3}{|c|}{$\mathrm{FTO} / \mathrm{TiO}_{2} / \mathrm{APTES} / \mathrm{Au} / \mathrm{Dye}$} & \multirow[b]{2}{*}{$\eta \%$} \\
\hline & $I_{\mathrm{sc}}$ & $\mathrm{V}_{\mathrm{oc}}$ & $\mathrm{FF}$ & \\
\hline 1 & 18.83 & 0.77 & 0.62 & 8.90 \\
\hline 2 & 18.80 & 0.76 & 0.61 & 8.74 \\
\hline 3 & 19.03 & 0.76 & 0.60 & 8.67 \\
\hline Ave & 18.89 & 0.76 & 0.61 & 8.77 \\
\hline \multirow[t]{2}{*}{ Stdev } & 0.13 & 0.01 & 0.01 & 0.12 \\
\hline & \multicolumn{2}{|c|}{$\mathrm{FTO} / \mathrm{TiO}_{2} / \mathrm{Dye}(\mathrm{NC}-1)$} & & \\
\hline S.N. & $\mathrm{I}_{\mathrm{sc}}$ & $\mathrm{V}_{\mathrm{oc}}$ & FF & $\eta \%$ \\
\hline 1 & 17.11 & 0.70 & 0.46 & 5.56 \\
\hline 2 & 15.94 & 0.68 & 0.68 & 7.37 \\
\hline 3 & 16.75 & 0.67 & 0.58 & 6.46 \\
\hline Ave & 16.60 & 0.68 & 0.57 & 6.46 \\
\hline Stdev & 0.60 & 0.02 & 0.11 & 0.91 \\
\hline
\end{tabular}

remove weakly bound dye. ${ }^{130}$ There is slightly higher dye coverage on the PAs with Au NPs as evidenced by the higher absorbance values of the MLCT peak for dye $\sim 530 \mathrm{~nm}$. 
Note that Au NPs do not contribute to the spectrum since they were background subtracted. The efficiency of the DSSC with Au NPs is higher $(8.8 \pm 0.1 \%)$ compared to the traditional DSSC (6.5 $\pm 0.9 \%)$. Table 6.3 summarizes the PEC parameters of the PAs. Modification with $\mathrm{Au}$ NPs increases both short circuit current, $\mathrm{J}_{\mathrm{sc}}$, open circuit voltage $\mathrm{V}_{\mathrm{oc}}$, and FF is also increased by about $6 \%$. The overall efficiency increased by $36 \%$ upon attachment of Au NPs through the APTES linker with this preparation method.

The DSSCs in Figure 6.5 were not optimized for highest efficiency. Literature reports show traditional DSSCs with efficiencies in the 9-10\% range. In order to reach that level with our traditional DSSCs, we deposited two layers of paste by the doctor blade method included a $\mathrm{TiO}_{2}$ scattering layer. As showing in Figure 6.6, our traditional DSSCs prepared with such conditions displayed an efficiency of $9.0 \%$, which is closer to those reported in the literature. When we prepared the PA the same way, but also attached Au NPs through the APTES linker before dye adsorption, we obtained a maximum efficiency of $10.4 \%$. Figure 6.6 shows I-V curves for all DSSCs prepared for this project. The increase is again the result of increased photocurrent and photovoltage. The statistics of photoanodes made in the lab is illustrated in Table 6.4. A bar plot for PEC parameters for all batches of PAs are shown in Figure 6.7. In most cases, AuNP- modified PAs showed the improvements in overall PEC values for samples prepared in the same day. The colors in Figure 6.6 are designated for batch number. The same color on both figures means same day preparation. Since the efficiency depends on dye coverage and the dye coverage is larger for the AuNP-modified DSSCs, we prepared PAs with the same dye loading to determine if there are other factors that lead to higher efficiency for the DSSCs with Au NPs. We used the metal-to-ligand charge transfer (MLCT) peaks in the UV-vis spectra as a measure of dye 
coverage and prepared all single layer $\mathrm{TiO}_{2}$ films without scattering layer. Figure 6.8A shows the UV-vis spectra of $\mathrm{FTO} / \mathrm{TiO}_{2} / \mathrm{Dye}, \mathrm{FTO} / \mathrm{TiO}_{2} / \mathrm{APTES} / \mathrm{Dye}$, and
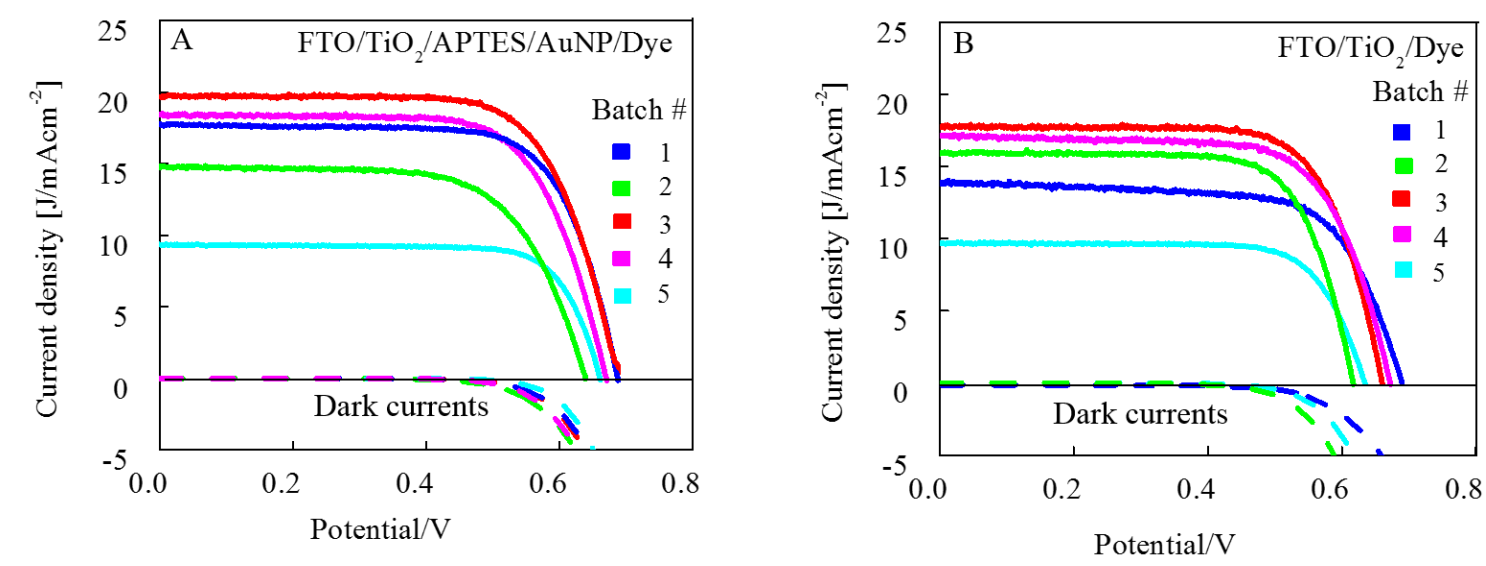

Figure 6.6. (A) Photocurrent-photovoltage plots by batch number for AuNP-modified photoanodes and (B) $\mathrm{TiO}_{2}$ photoanodes. Each batch contains two or three devices and the plots contain average values of I-V curve from each batch, Blue, green, red, pink, and cyan colors denoted batch numbers $1,2,3,4$, and 5, respectively.

Table 6.4. PEC parameters for all AuNP-modified and conventional PAs.

\begin{tabular}{|c|c|c|c|c|c|c|c|c|c|}
\hline \multicolumn{5}{|c|}{$\mathrm{FTO} / \mathrm{TiO}_{2} / \mathrm{APTES} / \mathrm{AuNP} / \mathrm{Dye}$} & \multicolumn{5}{|c|}{$\mathrm{FTO} / \mathrm{TiO}_{2} /$ Dye } \\
\hline S.N. & $\mathrm{J}_{\mathrm{sc}} / \mathrm{mAcm}^{-2}$ & $\mathrm{~V}_{\mathrm{oc}} / \mathrm{V}$ & $\eta \%$ & $\mathrm{FF}$ & S.N. & $\mathrm{J}_{\mathrm{sc}} / \mathrm{mAcm}^{-2}$ & $\mathrm{~V}_{\mathrm{od}} / \mathrm{V}$ & $\eta \%$ & FF \\
\hline $1 \mathrm{~A}$ & 18.06 & 0.69 & 8.59 & 0.69 & $1 \mathrm{~A}$ & 13.82 & 0.69 & 6.64 & 0.70 \\
\hline 1B & 18.75 & 0.66 & 8.77 & 0.71 & $2 \mathrm{~A}$ & 17.75 & 0.68 & 8.51 & 0.70 \\
\hline $2 \mathrm{~A}$ & 18.19 & 0.69 & 8.76 & 0.70 & $3 \mathrm{~A}$ & 17.19 & 0.65 & 7.26 & 0.65 \\
\hline $2 \mathrm{~B}$ & 17.19 & 0.69 & 8.48 & 0.71 & $4 \mathrm{~A}$ & 17.88 & 0.64 & 8.15 & 0.71 \\
\hline $2 \mathrm{C}$ & 18.00 & 0.68 & 8.80 & 0.71 & $4 \mathrm{~B}$ & 19.75 & 0.64 & 9.00 & 0.72 \\
\hline $3 \mathrm{~A}$ & 18.38 & 0.68 & 9.47 & 0.75 & $5 \mathrm{~A}$ & 9.73 & 0.64 & 4.59 & 0.74 \\
\hline $3 \mathrm{~B}$ & 20.25 & 0.70 & 10.40 & 0.74 & $6 \mathrm{~A}$ & 15.88 & 0.62 & 7.18 & 0.73 \\
\hline $3 \mathrm{C}$ & 20.31 & 0.66 & 9.33 & 0.70 & & & & & \\
\hline $4 \mathrm{~A}$ & 9.28 & 0.65 & 4.54 & 0.76 & & & & & \\
\hline $4 \mathrm{~B}$ & 9.50 & 0.68 & 4.91 & 0.76 & & & & & \\
\hline $5 \mathrm{~A}$ & 15.75 & 0.67 & 7.54 & 0.72 & & & & & \\
\hline $5 B$ & 13.94 & 0.67 & 6.95 & 0.75 & & & & & \\
\hline
\end{tabular}

Table of average PEC parameters by batch number FTO/TiO $2 /$ APTES/AuNP/Dye $\mathrm{FTO} / \mathrm{TiO}_{2} / \mathrm{Dye}$

\begin{tabular}{|c|c|c|c|c|c|c|c|c|}
\hline Batch\# & $\mathrm{J}_{\mathrm{sc}} / \mathrm{mAcm}^{-2}$ & $\mathrm{~V}_{\mathrm{oc}} / \mathrm{V}$ & $\eta \%$ & $\mathrm{FF}$ & $\mathrm{J}_{\mathrm{sc}} / \mathrm{mAcm}^{-2}$ & $\mathrm{~V}_{\mathrm{oc}} \mathrm{V}$ & $\eta \%$ & $\mathrm{FF}$ \\
\hline 1 & 18.41 & 0.68 & 8.68 & 0.70 & 13.82 & 0.69 & 6.64 & 0.70 \\
\hline 2 & 17.79 & 0.69 & 8.68 & 0.71 & 17.75 & 0.68 & 8.51 & 0.70 \\
\hline 3 & 19.65 & 0.68 & 9.73 & 0.73 & 18.27 & 0.64 & 8.14 & 0.69 \\
\hline 4 & 9.39 & 0.66 & 4.73 & 0.76 & 9.73 & 0.64 & 4.59 & 0.74 \\
\hline 5 & 14.85 & 0.67 & 7.25 & 0.74 & 15.88 & 0.62 & 7.18 & 0.73 \\
\hline
\end{tabular}

FTO/TiO 2 /APTES/AuNPs/Dye with the almost equal absorbance levels. This confirms that each PA was loaded with a similar amount of dye. The metal-to-ligand charge transfer (MLCT) peak of the dye on $\mathrm{FTO}_{\text {TiO}} / \mathrm{APTES}_{2}$ AuNPs/Dye is slightly more blue-shifted 
than that of $\mathrm{FTO} / \mathrm{TiO}_{2} / \mathrm{Dye}$, but slightly more red-shifted than that of FTO/TiO $/$ /APTES/Dye. The blue-shift on AuNP-modified PAs is due to the less interaction between $\mathrm{N} 719$ dye and the $\mathrm{TiO}_{2}$ NPs, nonetheless Au can interact with the dye and move
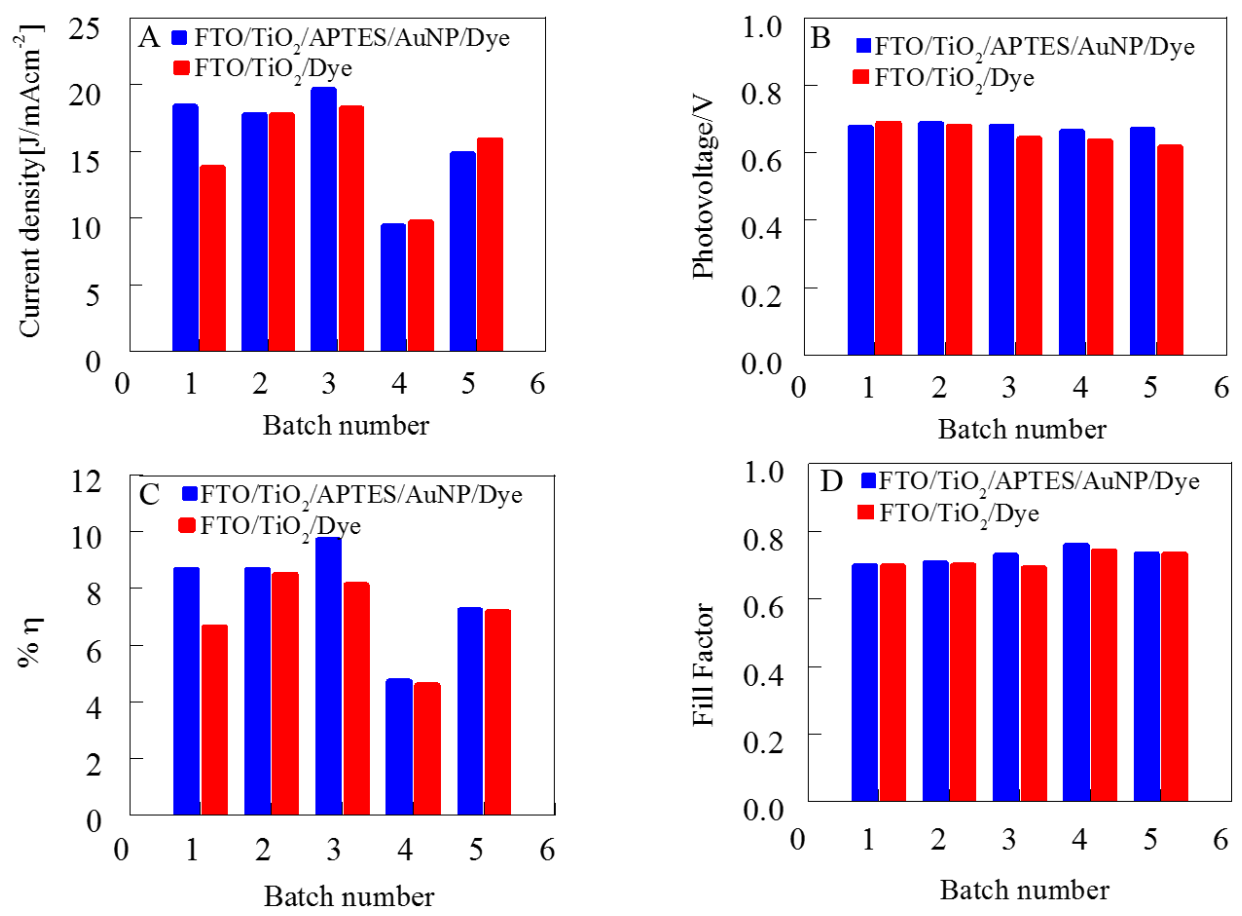

Figure 6.7. Bar plot of current density ( A), photovoltage (B), efficiency in percentage (C), and fill factor (Figure D) of linker assisted AuNP-modified photoanodes (blue) and $\mathrm{TiO}_{2}$ only photoanodes (red). Increase in efficiency of the devices is not only due to the enhancement of current but also due to the increase of photovoltage. A few devices have lower current densities, but, the other parameters $\left(\mathrm{V}_{\mathrm{oc}}, \eta\right.$, and $\left.\mathrm{FF}\right)$ remained the same.

the MLCT slightly red-shifted in comparison to $\mathrm{FTO} / \mathrm{TiO}_{2} / \mathrm{APTES} / \mathrm{Dye}$. We measured the incident to photon conversion efficiency (IPCE) of the same PAs shown in Figure 6.8B. The IPCE value was largest for the $\mathrm{FTO} / \mathrm{TiO}_{2} / \mathrm{APTES} / \mathrm{AuNPs} / \mathrm{Dye} \mathrm{PA}$, which was $~ 70 \%$ at $520 \mathrm{~nm}$. The IPCE value for the conventional PA was near $50 \%$ at $530 \mathrm{~nm}$, which is similar to the value reported in literature. ${ }^{233}$ The IPCE value for the FTO/TiO 2 /APTES PA was only $28 \%$ at $550 \mathrm{~nm}$. The I-V curves for the three PAs are shown in Figure 6.5C. The Au NPs modified PA showed the largest efficiency, which is $\sim 30 \%$ more than the traditional DSSC. Since the photon absorption by the dye is the same on all PAs, the increase in 
efficiency can be attributed to the properties of the Au NPs and not just to higher dye coverage in the earlier plots (Figures 6.5). Figure 6.9 shows the UV-vis peaks for desorbed

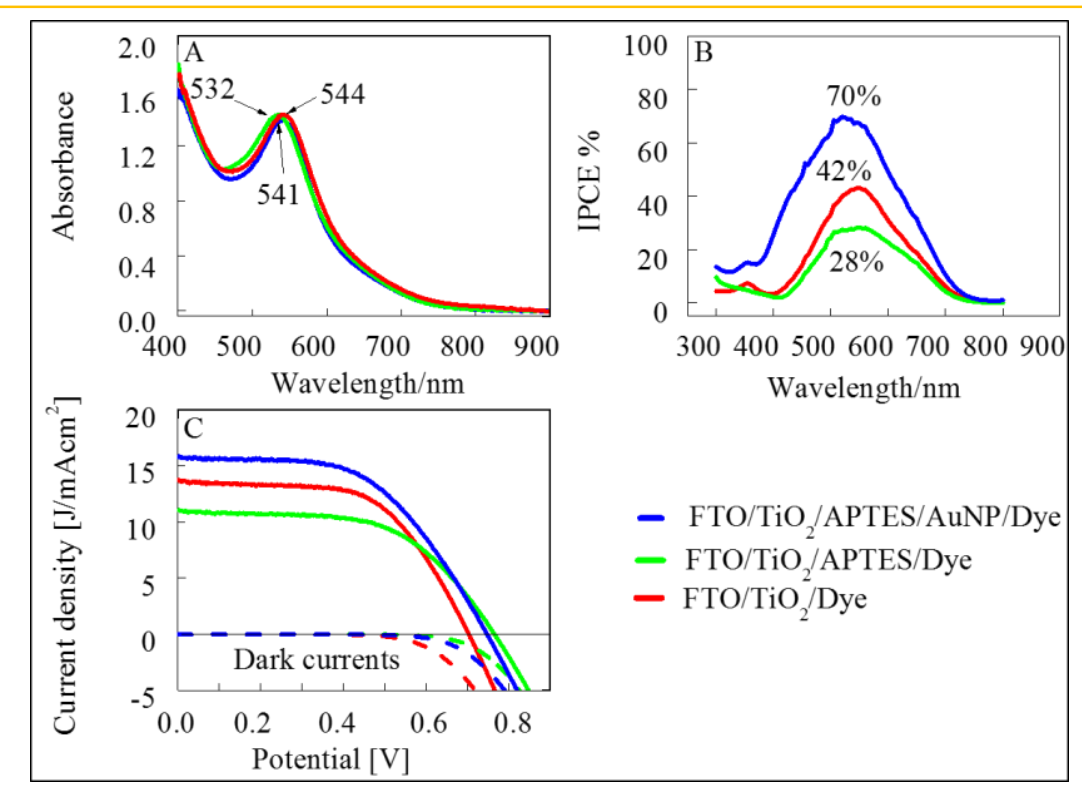

Figure 6. 8. Figure (A) UV-vis spectra of $\mathrm{FTO} / \mathrm{TiO}_{2} / \mathrm{Dye}$ (red), $\mathrm{FTO} / \mathrm{TiO}_{2} / \mathrm{APTES}_{\text {Dye }}$ (green), and $\mathrm{FTO} / \mathrm{TiO}_{2} / \mathrm{APTES} / \mathrm{AuNPs} / \mathrm{Dye}$ (blue) PAs, (B) IPCE of the corresponding photoanodes, and (C) I-V curves of the same devices. Dye-loading was the same for all PAs, and the area of all PAs was $0.30 \mathrm{~cm}^{2}$. The dash lines are dark I-V curves.

dye by using $0.05 \mathrm{M}$ aqueous solution of $\mathrm{KOH}$ and $\mathrm{I}-\mathrm{V}$ curves before desorption measured from Beaker cell of the similar types of PAs as shown in Figure 6.8. I-V curves in Figure 6.9B shows the increase in current for AuNP-modified PAs was $25 \%$. Again, the increase in efficiency can be attributed to the properties of the Au NPs incorporated into $\mathrm{TiO}_{2}$.

We obtained EIS data for the different PAs with the same dye coverage as shown in Figure 6.10. The resistance in the $\mathrm{TiO}_{2}$ mesoporous region, $\mathrm{R}_{\mathrm{ct}}$, was the largest for $\mathrm{FTO} / \mathrm{TiO}_{2} / \mathrm{APTES} / \mathrm{Dye} \mathrm{PAs}$ and lowest for $\mathrm{FTO} / \mathrm{TiO}_{2} /$ Dye PAs. The $\mathrm{R}_{\mathrm{ct}}$ is slightly higher for $\mathrm{FTO} / \mathrm{TiO}_{2} / \mathrm{APTES} / \mathrm{AuNPs} / \mathrm{Dye} \mathrm{PAs}$ compared to $\mathrm{FTO} / \mathrm{TiO}_{2} / \mathrm{Dye}$ PAs when the dye coverage is the same. The electron lifetime is related to the $1 /$ frequency value at the middle of the semicircle on the Nyquist plot. Consistent with a lower $R_{\mathrm{ct}}$, the $1 /$ frequency value was the smallest for the $\mathrm{FTO} / \mathrm{TiO}_{2} / \mathrm{Dye}_{\mathrm{PA}}$. It is difficult to conclude from EIS data, 
however, since $\mathrm{R}_{\mathrm{ct}}$ was larger for the AuNP-modified PAs at the same coverage, it is likely that the lower $\mathrm{R}_{\mathrm{ct}}$ value for the optimized PAs was due to the higher dye coverage and not
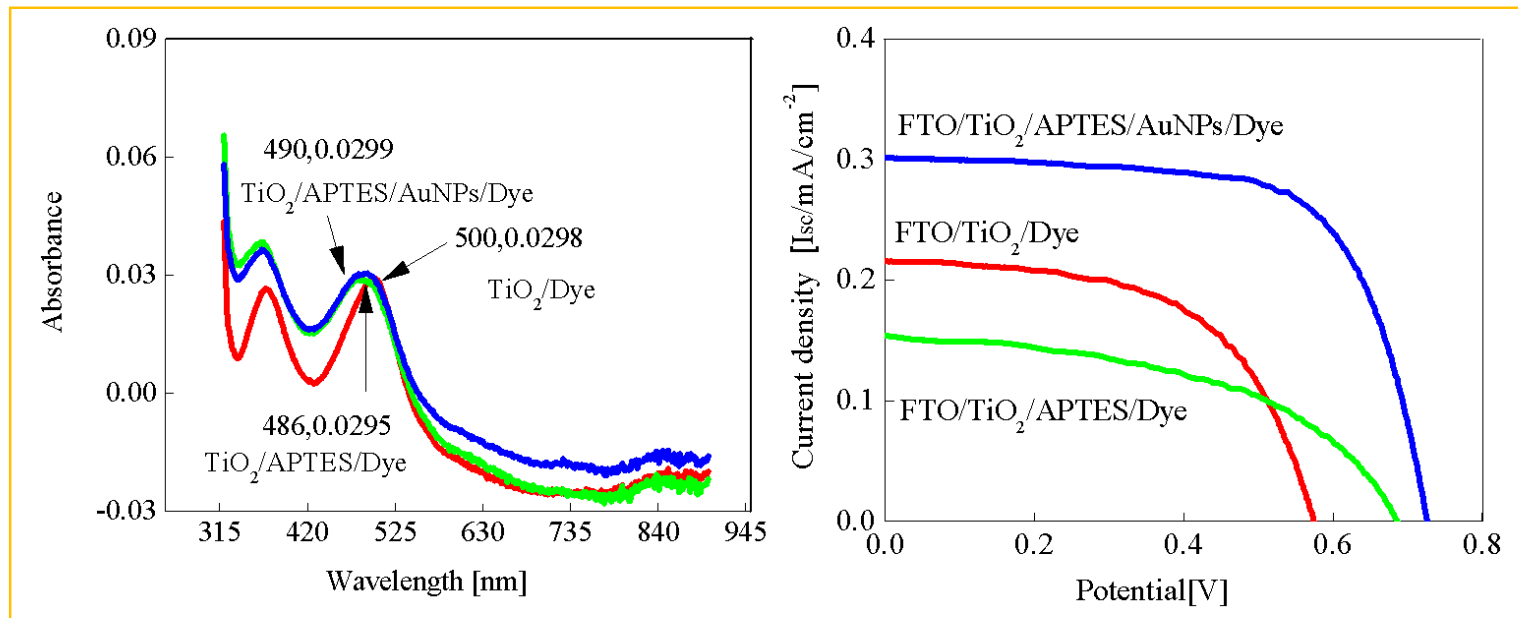

Figure 6.9. (A) UV-Vis of aliquots of $\mathrm{N} 719$ dye desorbed from $\mathrm{FTO} / \mathrm{TiO}_{2} / \mathrm{Dye}$ (red), $\mathrm{FTO} / \mathrm{TiO}_{2} \mathrm{APTES} / \mathrm{Dye}$ (green), and $\mathrm{FTO} / \mathrm{TiO}_{2} / \mathrm{APTES} / \mathrm{Au} / \mathrm{Dye}$ (blue). (B) I-V curves FTO/TiO 2 /Dye (red), FTO/TiO 2 APTES/Dye (green), and $\mathrm{FTO} / \mathrm{TiO}_{2} / \mathrm{APTES} / \mathrm{Au} / \mathrm{Dye}$ (blue). The I-V curves were measured from beaker cells.

an intrinsic property of the Au NPs that lowered the resistance of the $\mathrm{TiO}_{2}$ film.

To further our understanding of the role of Au NPs in the improved efficiency of DSSCs, we performed ultrafast transient absorption spectroscopy to investigate the electron injection process in all there PAs. The experimental apparatus was described in detail in Chapter 2 and 4. In brief, the photoinduced processes in the PAs were pumped at $530 \mathrm{~nm}$ and probed by a white light source and a near infrared source at $860 \mathrm{~nm}$. The transient absorption spectrum probed at $860 \mathrm{~nm}$ illustrates the excited-state kinetics of dye (Figure 6.11). The charge transfer dynamics did not change significantly for AuNP-modified PA (FTO/TiO $\left.2 / \mathrm{APTES}_{\mathrm{AuNP}} / \mathrm{Dye}\right)$ as compared to conventional PA $\left(\mathrm{FTO} / \mathrm{TiO}_{2} / \mathrm{Dye}\right)$. In both PAs, the time scale of the electron injection process from excited-state dye to $\mathrm{TiO}_{2}$ is below $50 \mathrm{fs}$. In comparison, the electron injection process is retarded in $\mathrm{FTO} / \mathrm{TiO}_{2} / \mathrm{APTES}_{\text {Dye }}$ (above $70 \mathrm{fs}$ ), but still significantly faster than charge recombination. Figure 6.11 shows a 
comparison of the injection rates for all three types of PAs with the slow component denoted by the magnitude of the baseline. The slow component of the decay kinetics is due to electron injection either from the triplet states of the dye ${ }^{135}$ or from multilayer dye (or weakly

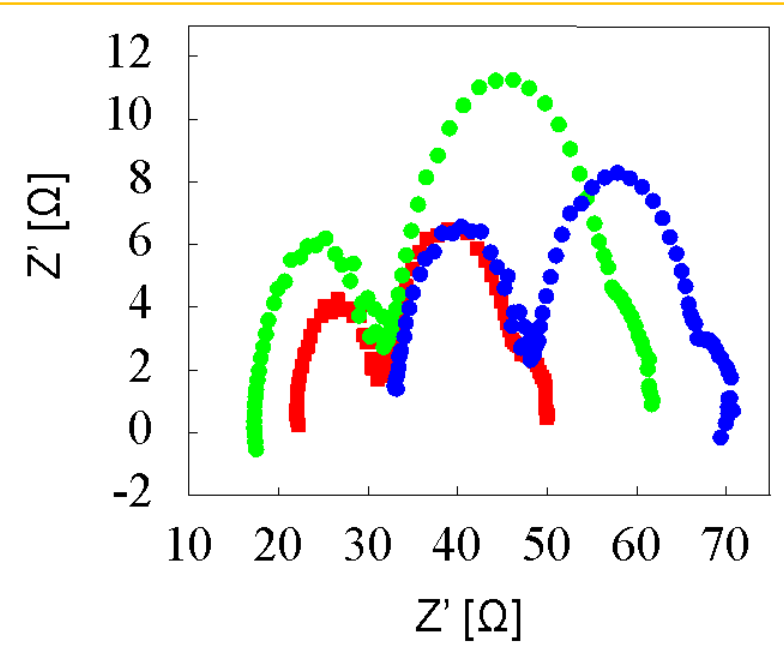

Figure 6.10. EIS plot for FTO/TiO $/$ /Dye (red solid square), FTO/TiO $/ 2$ APTES /Dye (green solid circle, and $\mathrm{FTO} / \mathrm{TiO}_{2} / \mathrm{APTES} / \mathrm{AuNP} /$ Dye (blue solid sphere) photoanodes having similar dye

coupled dye) molecules. ${ }^{234}$ A baseline close to zero in the decay kinetics of the Au NPmodified PA indicates that all dye molecules are active and strongly interacting with $\mathrm{TiO}_{2}$, and therefore contributing to the photoelectrochemical process. A non-zero baseline was observed in decay kinetics of the other two PAs. Such comparison suggests that Au NPs allow a greater percentage of dye molecules to be well-coupled to the $\mathrm{TiO}_{2}$ and participate in the electron injection process. A zero baseline means that this PA allows both higher dye coverage, and that almost all dye molecules are undergoing the electron injection process on the fs timescale. In contrast, the other PAs have smaller overall dye coverage along with a certain percentage of them not strongly coupled to $\mathrm{TiO}_{2}$ and therefore not contributing to the photocurrent. Those that contribute exhibit fs electron injection times, but not all dye molecules contribute. It is possible that the Au NPs facilitate long-range electron injection 
from weakly-coupled or multilayer dye molecules or aggregates, improving the overall efficiency.

Murakoshi revealed that strong orbital coupling between $\mathrm{N} 719$ and $\mathrm{TiO}_{2}$ increases $\mathrm{J}_{\mathrm{sc}}$ but decreases $\mathrm{V}_{\mathrm{oc}}$. This is because a large number of electrons in the $\mathrm{COO}^{-}$anchoring groups will be gathered after excitation of the dye by light. Because of the proximity of the

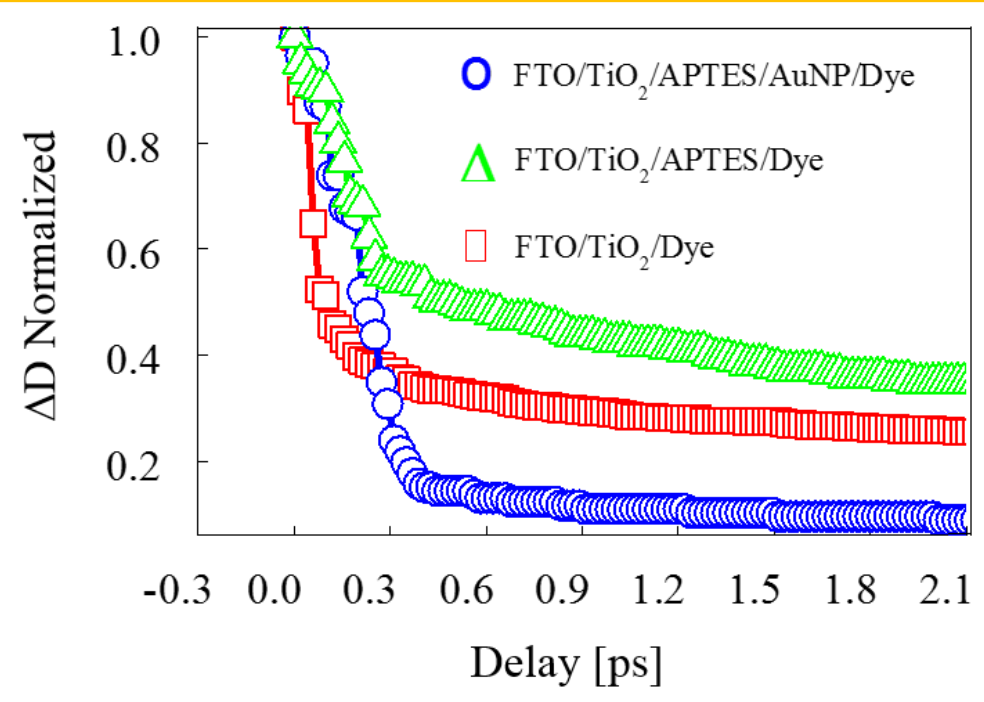

Figure 6.11. Comparisons of injection time scales for $\mathrm{FTO} / \mathrm{TiO}_{2} /$ Dye (red, traditional), FTO/TiO $/$ /APTES/Dye (green), and FTO/TiO $2 / \mathrm{APTES} / \mathrm{AuNPs} /$ Dye (blue). $\mathrm{T}$ denotes the ultrafast time scale for excited electrons to go from LUMO to conduction band of $\mathrm{TiO}_{2}$. The ultrafast injection dynamics are the fastest in Au NPs modified photoanodes.

$$
\begin{array}{ccc}
\mathrm{T}_{\mathrm{TiO} 2 / \mathrm{APTES} / \mathrm{Au} / \mathrm{Dye}}> & \mathrm{T}_{\mathrm{TiO} / \mathrm{Dye}}>\mathrm{T}_{\mathrm{TiO} / \mathrm{APTES} / \mathrm{Dye}} \\
<38 \mathrm{fs} & <48 \mathrm{~s} & <73 \mathrm{fs}
\end{array}
$$

conduction band (CB) and LUMO of N719 dye, the electron injection rates will also increase upon photo-irradiation. The electron injection process produces a large number of electrons in the $\mathrm{TiO}_{2} \mathrm{CB}$ within a very short period of time, which, in turn, increases the probability of recombination with the electrolyte and dye itself. The introduction of APTES in between $\mathrm{TiO}_{2}$ and dye creates an indirect interaction with the dye. This may inhibit charge recombination with $\mathrm{TiO}_{2}$ and electrolyte, which, in turn, increases the Fermi level. This 
increases $V_{o c}$ as observed in our results from PAs with the APTES linker (with and without Au NPs). ${ }^{100}$

The FTO/TiO 2 /APTES/Dye has a higher $\mathrm{V}_{\text {oc }}$ compared to the traditional FTO/TiO 2 /Dye PA, but a lower $\mathrm{J}_{\mathrm{sc}}$. The lower $\mathrm{J}_{\mathrm{sc}}$ is likely due to a larger percentage of dye molecules weakly coupled or aggregated on the $\mathrm{TiO}_{2}$ surface and not participating in the electron injection process, as determined by transient absorption measurements. By attaching the $\mathrm{Au}$ NPs to the APTES layer, the device gets the benefit from the increased $V_{\text {oc }}$ due to the APTES layer and a larger amount of dye loading. More importantly, a larger percentage of dye molecules are involved in the electron injection process, as indicated by the almost zero baseline in the transient absorption spectrum.

Previous reports have shown that Au NPs may also increase the Fermi-level in the interfacial region, which helps increase the driving force of the system and increases the value of $\mathrm{V}_{\mathrm{oc}}{ }^{222}$ Plasmonic Au NPs are also known to help transfer charge over a long range up to Foster resonance distances. ${ }^{223}$ Dark current measurement can determine the recombination rates in the real devices because a higher bias potential required to produce

dark current means higher blockage power for recombination. ${ }^{235}$ In those devices, $V_{\mathrm{oc}}$ increased under operating conditions. The conventional $\mathrm{FTO} / \mathrm{TiO}_{2} / \mathrm{Dye} \mathrm{PA}$ required a lower bias voltage to produce a dark current compared to $\mathrm{FTO} / \mathrm{TiO}_{2} / \mathrm{APTES} / \mathrm{Dye}$ and FTO/TiO 2 /APTES/Au/NP/Dye PAs, consistent with aforementioned hypothesis.

\subsection{Conclusions}

The effect of attaching plasmonic Au NPs on PAs used for DSSCs has been studied. A molecular linker that can interact strongly with Au NPs and form a monolayer on the 
metal oxide facilitates long-range charge transfer processes in the interfacial region. The use of a molecular linker can be conducive to optimize device performance thanks to the improvements of three main aspects: suppression of back reaction from back contact (FTO), reduction in recombination of electrons in $\mathrm{TiO}_{2}$ to electrolyte, and increasing driving force by increasing the Fermi-level of electrons in $\mathrm{TiO}_{2}$. An alkyl chain of APTES can provide a one-way channel for electron transfer (ET) from excited dye to back contact (FTO). Additionally, APTES can equally react with exposed and non-exposed parts of FTO. Therefore, ET from FTO to electrolyte can be inhibited, which can be clearly observed in the increase in the open circuit photovoltage $\left(\mathrm{V}_{\text {oc }}\right)$. Without Au NPs, FTO/TiO $/$ APTES/Dye PAs have a current lower than that of the conventional PAs. However, having Au NPs attached on APTES increased $J_{s c}$ as well as $V_{o c}$, though $V_{o c}$ is slightly less than that of APTES alone. Attaching Au NPs on APTES is an easy and reproducible process and hence a feasible method to improve the efficiency of DSSCs. Attaching the Au NPs to the outer surface chemically is highly controlled and offers the potential to learn the fundamentals of the process.

Ultrafast transient absorption spectroscopy shows less effect Au NPs on the injection dynamics than devices with linkers only, which is required for the optimization of PCE parameters. This research can be extended to other dyes which can combine strongly with plasmonic Au NPs and replace Ru-complexes since it is a rare earth metal. Au is corrosive to $\mathrm{I}^{-} / \mathrm{I}_{3}{ }^{-}$, therefore, an alternate electrolyte eco-friendly to Au will be required in the future. 


\section{CHAPTER 7}

\section{GLOBAL CONCLUSIONS AND FUTURE DIRECTIONS}

\subsection{Surface Chemistry}

Stable and efficient dye-sensitized solar cells were prepared utilizing the principle of surface modification for $\mathrm{TiO}_{2}$ nanoparticles $(15-35 \mathrm{~nm})$ with organic modifiers such as APTES and APhS. DCC and DMAP can induce the formation of an amide bond between the $\mathrm{COOH} / \mathrm{COO}^{-}$anchor group of $\mathrm{N} 719$ dye and the $\mathrm{NH}_{2}$ formed on $\mathrm{TiO}_{2}$. In Chapter 3 , it is found that covalent amide bonding of N719 through silane linker to $\mathrm{TiO}_{2}$ improves stability to air moisture, heat, UV light, and acid in comparison to traditional photoanodes. The improvement is related to the preservation of SCN ligand on N719 on covalently-linked PAs. The covalent linkage results in the formation of multilayer dye that lowers efficiency under normal soaking time (24 h). Removal of multilayers of dye by acid or by soaking less time in dye solution can increase the efficiency close to traditional photoanodes. This PA with covalently-linked dye can offer better advantage of being stable structure. The photovoltage and fill factor were improved in spite of a little decrease in photocurrent. This is an advantage of covalently-linked PAs.

Chapter 4 showed the fundamental insight of charge dynamics in covalent and conventional (non-covalent) PAs. Electron injection is slower in covalent photoanodes than conventional; however, it is still faster than the charge recombination to dye molecules and 
the electrolyte as well. Photoanodes covalently-linked with aromatic linkers showed faster injections dynamics and improved PCE. The covalently-linked N719 dye with aromatic linker also showed the same stability towards heat, water, and UV light. Multilayer formation is still an issue but can be solved by controlling the time of soaking for covalent photoanodes.

\subsection{Combination of Bulk and Nanoparticles}

Chapter 5 showed the profound enhancement of photocurrent by $20-30 \%$ as compared to traditional PAs when micro-sized $\mathrm{Nd}_{2} \mathrm{O}_{3}$ particles were doped into $\mathrm{TiO}_{2}$ in the ratio of 20:1. In spite of drastically changing the morphology of the surface of $\mathrm{TiO}_{2}$ by $\mathrm{Nd}_{2} \mathrm{O}_{3}$ creating several voids and cracks as observed in SEM images, the UV-vis shows higher dye-coverage on those Nd-doped samples. The samples containing only $\mathrm{Nd}_{2} \mathrm{O}_{3}$ did not show any affinity towards the dye. Impedance spectroscopy shows that there is a possibility of removing trap states of the $\mathrm{TiO}_{2}$ nanosurface by $\mathrm{Nd}_{2} \mathrm{O}_{3}$, because sample doped with $5 \% \mathrm{Nd}_{2} \mathrm{O}_{3}$ showed reduced internal resistance. Diffuse reflectance spectroscopy showed the electronic transitions from f-states of $\mathrm{Nd}_{2} \mathrm{O}_{3}$ in the nano-micro composite mesoporous films. When energy levels are compared betweeen $\mathrm{TiO}_{2}$ and $\mathrm{Nd}_{2} \mathrm{O}_{3}$, the f-states are near the trap states of $\mathrm{TiO}_{2}$. Similar dye-coverage samples doped with $5 \% \mathrm{Nd}_{2} \mathrm{O}_{3}$ gave the higher IPCE values than the undoped samples, also consistent with the improved efficiency. 


\subsection{Nanostructured Materials}

Today's world is a world of nanostructured materials. Nanostructured materials are applicable to many areas of research such as physics, chemistry, engineering, biology, and medicine. Chapter 6 described the fabrication of high-efficiency devices by electrostatically attaching negatively-charged gold nanoparticles $(\sim 3-5 \mathrm{~nm}$ in diameter) to protonated amines formed on the surface of $\mathrm{TiO}_{2}$. This technique is unique because it improved the overall photoelectrochemical parameters such as photocurrent density $(\mathrm{j})$, photovoltage $\left(\mathrm{V}_{\mathrm{oc}}\right)$, fill factor (FF), and efficiency ( $\eta \%)$. APTES inhibits charge recombination, whereas Au NPs can facilitate a faster injection rate from $\mathrm{N} 719$ dye to $\mathrm{TiO}_{2}$ at long range distances. In addition, Au NPs can harvest more light unabsorbed by N719 because Au NPs have a plasmon absorption band in the red light region where the dye absorbs light.

\subsection{Plasmon Enhanced Charge Transfer Dynamics at Nanoscale}

Charge transfer dynamics in DSSCs using ultrafast transient absorption spectroscopy (UTAS) was discussed in Chapters 4 and 6. The photoanodes were first excited by $530 \mathrm{~nm}$ laser light and the fate of the excited electrons were probed by $860 \mathrm{~nm}$ laser light. Ultrafast injection dynamics were the fastest in AuNP- modified photoanodes in the order:

$$
\begin{aligned}
& \mathrm{TTiO}_{\mathrm{Ti} / \mathrm{APTS} / \mathrm{Au} / \mathrm{Dye}}> \mathrm{T}_{\mathrm{TiO} / \mathrm{Dye}}>\mathrm{T}_{\mathrm{TiO} 2 / \mathrm{APTES} / \mathrm{Dye}} \\
&<38 \mathrm{fs} \quad<48 \mathrm{~s} \quad<73 \mathrm{fs}
\end{aligned}
$$

where $t$ denotes the injection time scale of excited electron from dye to the $\mathrm{TiO}_{2}$ conduction band.

In decay dynamics, the baseline curve is due to slow components of charge injection from N719 dye to the conduction band of the $\mathrm{TiO}_{2}$; these are either from the triplet states of 
the dye or from the multilayer dye (or weakly coupled dye) molecules. A baseline close to zero in the decay kinetics of the AuNP-modified PAs indicates that all dye molecules are actively and strongly interacting with $\mathrm{TiO}_{2}$, and therefore contributing to the photoelectrochemical processes. A non-zero baseline was observed in the decay kinetics of the other two types of PAs, $\mathrm{TiO}_{2} / \mathrm{N} 719$ and $\mathrm{TiO}_{2} / \mathrm{APTES} / \mathrm{N} 719$. Such comparison suggests that $\mathrm{Au}$ NPs allow a greater percentage of dye molecules to be well-coupled to the $\mathrm{TiO}_{2}$ and participate in the electron injection process. The fastest injection dynamics are not always correlated to an increase in photocurrent. In Chapter 4, when an ester bond was created in $\mathrm{TiO}_{2}$ by treating with DCC and DMAP in the dye solution in dichloromethane, the injection dynamics were among the fastest, but the PCE value and stability were less for the devices. The baseline level correlated best with the PCE value, where a lower baseline equated to higher efficiency.

\subsection{Future Directions}

- Traditionally-prepared PAs in a sandwich cell are not stable indefinitely. In Chapter 3 it was shown that the PAs prepared with covalently-attached dye can be expected to have better stability in sealed sandwich cells since degradation by UV, heat, acid, and water may play a role for traditional PAs. The approach developed in this study for attaching N719 dye covalently through APTES allows for air storable PAs, which can provide added convenience and lower cost when considering the bulk-scale production of DSSCs. In the future, a detailed understanding of the reason for the added stability observed in covalently-attached PAs can be performed to improve the efficiency of the covalently modified PAs further. In addition, APTES is a 
common linker, but cannot always make a full monolayer coverage on $\mathrm{TiO}_{2}$. Future research will be oriented to look for new modifiers and apply them to semiconductor and quantum dot particles to explore other materials for energy related research.

- Modification of the covalently-attached photoanodes, for instance, with $\mathrm{Au}$ nanoparticles, can further improve the performance of DSSCs, which will be another of subject of future work. Furthermore, surface modification can be a potential technique to increase the dye coverage on different architectural nanomaterials such as nanowires, nanotubes, and nanosheets. These shapes and sizes are showing promising results in charge transfer dynamics, but the dye coverages are currently too low.

- APTES interacts electrostatically with plasmonic Au NPs, attaching them to the $\mathrm{TiO}_{2}$. Au NPs can facilitate long-range charge transfer processes in the interfacial region from $\mathrm{N} 719$ to $\mathrm{TiO}_{2}$. The use of molecular linkers can be conducive to optimize device performance due to the improvements in three main aspects: suppression of back reaction from back contact (FTO), reduction in recombination of electrons in $\mathrm{TiO}_{2}$ to electrolyte, and increasing driving force by increasing the Fermi-level of electrons in $\mathrm{TiO}_{2}$. An alkyl chain of APTES with Au NPs can provide a one-way channel for electron transfer (ET) from excited dye to the $\mathrm{TiO}_{2}$ and back contact (FTO), but block backward recombination. Additionally, APTES can react with $\mathrm{TiO}_{2}$ and exposed parts of FTO. Therefore, ET from FTO to electrolyte can be inhibited, which is clearly observed in the increase in the open-circuit photovoltage $\left(\mathrm{V}_{\mathrm{oc}}\right)$. Without Au NPs, $\mathrm{FTO} / \mathrm{TiO}_{2} / \mathrm{APTES} /$ Dye PAs have a current lower than that of the conventional PAs. Attaching Au NPs onto APTES increases the current and 
improves the efficiency of DSSCs because they can improve light harvesting capacity due to their plasmonic nature, increase dye-loading on devices, and make all dye molecules contribute to the injection dynamics. This research can be extended to other dyes which can combine strongly Au NPs, in order to replace Ru-complexes, since it is a rare earth metal. However, Au is corrosive to $\mathrm{I}^{-} / \mathrm{I}_{3}{ }^{-}$; therefore, an alternate electrolyte inert to Au NPs could be an interest of research in the future. Different shapes and sizes of $\mathrm{Au}$ or $\mathrm{Ag} \mathrm{NPs}$ that can be linked chemically to $\mathrm{TiO}_{2}$ or other semiconductor oxides could also be explored and applied to DSSCs.

- The combination of micro- and nano- particles in a composite film can lead to an important material for energy production. The size and shape of $\mathrm{Nd}_{2} \mathrm{O}_{3}$ in the study of DSSCs has not been fully explored, but should change the physical and chemical properties of the material and find use in energy applications, and water-splitting processes to produce $\mathrm{H}_{2}$ as a fuel. The surface property, such as porosity as a function of the size of $\mathrm{Nd}_{2} \mathrm{O}_{3}$ will be characterized in the future.

- Since Nd-doped samples facilitate the charge transport in the photoanode region, the combination of $\mathrm{Nd}_{2} \mathrm{O}_{3}$ with $\mathrm{TiO}_{2}$ can also be a good substrate for perovskite based solar cells, a recently emerging technology in the energy field. 


\section{REFERENCES}

(1) Gerland, P.; Raftery, A. E.; Sevcikova, H.; Li, N.; Gu, D.; Spoorenberg, T.; Alkema, L; Fosdick, B. K.; Chunn, J.; Lalic, N.; Bay, G.; Buettner, T.; Heilig, G. K.; Wilmoth, J. Science 2015, 346, 234.

(2) Lewis, N. S.; Crabtree, G.; Nozik, A. J.; Wasielewski, M. R.; Alivisatos, P. Report of the Basic Energy Sciences Workshop on Solar Energy Utilization 2005, 1.

(3) Adamantiades, A. Energy Policy 2009, 37, 5149.

(4) Kostic, M. M. Encyclopedia of Energy Engineering 2007.

(5) Islam, M. T.; Shahir, S. A.; Uddin, T. M. I.; Saifullah, A. Z. A. Renewable Sustainable Energy Rev. 2014, 39, 1074.

(6) Shafiee, S.; Topal, E. Energy Policy 2009, 37, 181.

(7) Schiermeier, Q., Tollefson, J., Scully, T., Witze, A., Morton, Q. Nature 2008, 454, 816.

(8) Hagfeldt, A.; Boschloo, G.; Sun, L.; Kloo, L.; Pettersson, H. Chem. Rev. 2010, 110, 6595.

(9) Saga, T. Npg Asia Materials 2010, 2, 96.

(10) Snaith, H. J. Adv. Funct. Mater. 2010, 20, 13.

(11) Hardin, B. E.; Snaith, H. J.; McGehee, M. D. Nat. Photonics 2012, 6, 162.169.

(12) Burschka, J.; Pellet, N.; Moon, S. J.; Humphry-Baker, R.; Gao, P.; Nazeeruddin, M. K.; Gratzel, M. Nature 2013, 499, 316.

(13) Chiba, Y., Islam, A., Watanabe, Y., Komiya, R., Koide N., Han, L. Jpn. J. Appl. Phys. 2006, 45, L638.

(14) Yella, A.; Lee, H.-W.; Tsao, H. N.; Yi, C.; Chandiran, A. K.; Nazeeruddin, M. K.; Diau, E. W.-G.; Yeh, C.-Y.; Zakeeruddin, S. M.; Grätzel, M. Science 2011, 334, 629.

(15) Koops, S. E.; Barnes, P. R. F.; O’Regan, B.; Durrant, J. R. J. Phys. Chem. C 2010, 114,8054 .

(16) Becquerel, E. C. R. Acad. Sci. Paris 1839, 561.

(17) Moser, J. E. Monatshe. Chem. 1887, 373. 
(18) http://astsun.astro.virginia.edu/Brjp0i/museum/photography.html.

(19) Fujishima, A.; Honda, K. Nature 1972, 238, 37.

(20) Tsubomura, H.; Matsumura, M., Nomura, Y., Amamiya, T. Nature 1976, 261, 402.

(21) O'Regan, B.; Grätzel, M. Nature 1991, 353, 737.

(22) Kalyansundaram, K. Dye-sensitized solar Cells 2010, 83.

(23) Zaban, A.; Chen, S. G.; Chappel, S.; Gregg, B. A. Chem. Commun. 2000, 2231.

(24) Nelson, J. The Physics of Solar cells, 2003.

(25) Hara, K.; Koumura. N. Mater. Matters 2009, 4, 92.

(26) Koumura, N.; Wang, Z.-S.; Miyashita, M.; Uemura, Y.; Sekiguchi, H.; Cui, Y.; Mori, A.; Mori, S.; Hara, K. J. Mater. Chem. 2009, 19, 4829.

(27) Nazeeruddin, M. K.; Kay, A.; Rodicio, I.; Humphry-Baker, R.; Mueller, E.; Liska, P.; Vlachopoulos, N.; Graetzel, M. J. Am. Chem. Soc. 1993, 115, 6382.

(28) Brown, P.; Takechi, K.; Kamat, P. V. J. Phys. Chem. C 2008, 112, 4776.

(29) Wang, M.; Anghel, A. M.; Marsan, B.; Ha, N.-L. C.; Pootrakulchote, N.; Zakeeruddin, S. M.; Graetzel, M. J. Am. Chem. Soc. 2009, 131, 15976.

(30) Gratzel, M. J. Photochem. Photobiol., C 2003, 4.

(31) Grätzel, M. C. R. Chim. 2006, 9, 578.

(32) Brauer, G. Handbook of Preparative Inorganic Chemistry, 1963, 1055.

(33) Ito, S.; Chen, P.; Comte, P.; Nazeeruddin, M. K.; Liska, P.; Pechy, P.; Grätzel, M. Prog. Photovoltaics 2007, 15, 603.

(34) Langmuir, I. Chem. Rev. 1933, 13, 147.

(35) Beeram, S. R.; Zamborini, F. P. Biosensing. J. Am. Chem. Soc.2009, 131, 11689.

(36) Kazuhito, H.; Hiroshi, I.; Akira, F. Jpn. J. Appl. Phys. 2005, 44, 8269.

(37) Wang, S. S.; Tam, J. P.; Wang, B. S. H.; Merrifield, R. B. Int. J. Pept. Protein Res. 1981, 18, 459 .

(38) Mieszawska, A. J.; Slawinski, G. W.; Zamborini, F. P. J. Am. Chem.l Soc. 2006, 128, 5622.

(39) Howarter, J. A.; Youngblood, J. P. Langmuir 2006, 22, 11142.

(40) Atwater, H. A.; Polman, A. Nat. Mate r. 2010, 9, 205.

(41) Nazeeruddin, M. K.; Splivallo, R.; Liska, P.; Comte, P.; Gratzel, M. Chem. Commun. 2003, 1456. 
(42) Gratton, E.; Fantini, S. Chapperonins 2004, 11, 211.

(43) Binnig, G.; Quate, C. F.; Gerber, Phys. Rev. Lett. 1986, 56, 930.

(44) Chan, K. L. A.; Hammond, S. V.; Kazarian, S. G. Anal. Chem. 2003, 75, 2140.

(45) Judith, A. S. Phys. Educ.1982, 17, 111.

(46) Bard, A. J. Electrochemical Methods: Fundamentals and Application 2001, 383.

(47) Wang, Q.; Moser, J.-E.; Grätzel, M. J. Phys. Chem. B 2005, 109, 14945.

(48) Usermanual: Alphastep 500 Surface Profilometer 1993.

(49) Snaith, H. J. Energy Environ. Sci. 2012, 5, 6513.

(50) Zewail, A. H. Pure appl. chem. 2000, 72, 2219.

(51) Berera, R.; van Grondelle, R.; Kennis, J. T. M. Photosynth. Res. 2009, 101, 105.

(52) Anderson, N. A.; Lian, T. Coord. Chem. Rev. 2004, 248, 1231.

(53) Boschloo, G.; Hagfeldt, A. Acc. Chem. Res. 2009, 42, 1819.

(54) Rowley, J. G.; Ardo, S.; Sun, Y.; Castellano, F. N.; Meyer, G. J. J. Phys. Chem. C 2011, 115, 20316.

(55) Xue, G.; Guo, Y.; Yu, T.; Guan, J.; Yu, X.; Zhang, J.; Liu, J.; Zou, Z. Int. J. Electrochem. Sci. 2012, 7, 1496.

(56) Ardo, S.; Meyer, G. J. Chem. Soc. Rev.2009, 38, 115.

(57) Zhao, Z.; Liu, H.; Chen, S. Nanoscale 2012, 4, 7301.

(58) Polo, A. S.; Itokazu, M. K.; Murakami Iha, N. Y. Coord. Chem. Rev. 2004, 248, 1343.

(59) Hirose, F.; Kuribayashi, K.; Shikaku, M.; Narita, Y.; Takahashi, Y.; Kimura, Y.; Niwano, M. J. Electrochem. Soc. 2009, 156, B987.

(60) Splan, K. E.; Massari, A. M.; Hupp, J. T. J. Phys. Chem. B 2004, 108, 4111.

(61) Wen, P.; Xue, M.; Ishikawa, Y.; Itoh, H.; Feng, Q. ACS Appl. Mater. Interfaces 2012, 4, 1928.

(62) Nazeeruddin, M. K.; Humphry-Baker, R.; Liska, P.; Grätzel, M. J. Phys. Chem. B 2003, 107, 8981 .

(63) Hirata, N.; Lagref, J.-J.; Palomares, E. J.; Durrant, J. R.; Nazeeruddin, M. K.; Gratzel, M.; Di Censo, D. Chem. Euro. J. 2004, 10, 595.

(64) Kilsa, K.; Mayo, E. I.; Brunschwig, B. S.; Gray, H. B.; Lewis, N. S.; Winkler, J. R. J. Phys. Chem. B 2004, 108, 15640. 
(65) Galoppini, E.; Guo, W. Z.; Qu, P.; Meyer, G. J. J. Am. Chem. Soc. 2001, 123, 4342.

(66) Kilsa, K.; Mayo, E. I.; Kuciauskas, D.; Villahermosa, R.; Lewis, N. S.; Winkler, J. R.; Gray, H. B. J. Phys. Chem. A 2003, 107, 3379.

(67) Kroeze, J. E.; Hirata, N.; Koops, S.; Nazeeruddin, M. K.; Schmidt-Mende, L.; Grätzel, M.; Durrant, J. R. J. Am. Chem. Soc 2006, 128, 16376.

(68) Jing, B. W.; Zhang, H.; Zhang, M. H.; Lu, Z. H.; Shen, T. J. Mater. Chem. 1998, 8, 2055.

(69) Haque, S. A.; Palomares, E.; Cho, B. M.; Green, A. N. M.; Hirata, N.; Klug, D. R.; Durrant, J. R. J. Am. Chem. Soc. 2005, 127, 3456.

(70) Perez Leon, C.; Kador, L.; Peng, B.; Thelakkat, M. J. Phys. Chem. B 2006, 110, 8723.

(71) Finnie, K. S.; Bartlett, J. R.; Woolfrey, J. L. Langmuir 1998, 14, 2744.

(72) Hirva, P.; Haukka, M. Langmuir 2010, 26, 17075.

(73) Heimer, T. A.; D'Arcangelis, S. T.; Farzad, F.; Stipkala, J. M.; Meyer, G. J. Inorg. Chem. 1996, 35, 5319.

(74) Gillaizeau-Gauthier, I.; Odobel, F.; Alebbi, M.; Argazzi, R.; Costa, E.; Bignozzi, C. A.; Qu, P.; Meyer, G. J. Inorg. Chem. 2001, 40, 6073.

(75) Park, H.; Bae, E.; Lee, J.-J.; Park, J.; Choi, W. J. Phys. Chem. B 2006, 110, 8740.

(76) Brown, D. G.; Schauer, P. A.; Borau-Garcia, J.; Fancy, B. R.; Berlinguette, C. P. J. Am. Chem. Soc. 2013, 135, 1692.

(77) Christ, C. S.; Yu, J.; Zhao, X.; Palmore, G. T. R.; Wrighton, M. S. Inorg. Chem. 1992, 31, 4439 .

(78) Ford, W. E.; Rodgers, M. A. J. J. Phys. Chem. 1994, 98, 3822.

(79) Ghosh, P.; Spiro, T. G. J. Am. Chem. Soc. 1980, 102, 5543.

(80) Hohman, J. R.; Fox, M. A. J. Am. Chem. Soc. 1982, 104, 401.

(81) Dabestani, R.; Bard, A. J.; Campion, A.; Fox, M. A.; Mallouk, T. E.; Webber, S. E.; White, J. M. J. Phys. Chem. 1988, 92, 1872.

(82) Fox, M. A.; Nobs, F. J.; Voynick, T. A. J. Am. Chem. Soc. 1980, 102, 4036.

(83) Fujihira, M.; Ohishi, N.; Osa, T. Nature 1977, 268, 226.

(84) Arzoumanian, H.; Castellanos, N. J.; Martínez, F. O.; Paez-Mozo, E. A.; Ziarelli, F. Eur. J. Inorg. Chem. 2010, 1633. 
(85) Bradley, J. B.; Amy, E. K.; Paul, A. L.; Sean, A. V.; Thomas, A. M.; Ana, L. M.; Devens, G. Nanotechnology 2009, 20, 505203.

(86) Franking, R. A.; Landis, E. C.; Hamers, R. J. Langmuir 2009, 25, 10676.

(87) Benson, M. C.; Ruther, R. E.; Gerken, J. B.; Rigsby, M. L.; Bishop, L. M.; Tan, Y.; Stahl, S. S.; Hamers, R. J. ACS Appl. Mater. Interfaces 2011, 3, 3110.

(88) Figgermeier, E., Hagfeldt, A. Int. J. Photoenergy 2004, 6, 127.

(89) Hinsch, A.; Kroon, J. M.; Kern, R.; Uhlendorf, I.; Holzbock, J.; Meyer, A.; Ferber, J. Prog. Photovoltaics 2001, 9, 425.

(90) Lim, J.; Kwon, Y. S.; Park, S.-H.; Song, I. Y.; Choi, J.; Park, T. Langmuir 2011, 27, 14647.

(91) Kohle, O.; Grätzel, M.; Meyer, A. F.; Meyer, T. B. Adv. Mater. 1997, 9, 904.

(92) Grünwald, R.; Tributsch, H. J. Phys. Chem. B 1997, 101, 2564.

(93) Nguyen, H. T.; Ta, H. M.; Lund, T. Sol. Energy Mater. Sol. Cells 2007, 91, 1934.

(94) Greijer Agrell, H.; Lindgren, J.; Hagfeldt, A. Sol. Energy 2003, 75, 169.

(95) Turrion, M.; Macht, B.; Salvador, P.; Z. Phys. Chem. 1999, 212, 51.

(96) Heo, N.; Jun, Y.; Park, J. H. Sci. Rep. 2013, 3.

(97) Vinodgopal, K.; Hua, X.; Dahlgren, R. L.; Lappin, A. G.; Patterson, L. K.; Kamat, P. V. J. Phys. Chem. 1995, 99, 10883.

(98) Mieszawska, A. J.; Slawinski, G. W.; Zamborini, F. P. J. Am. Chem. Soc. 2006, 128, 5622.

(99) Chun-Feng, L.; Zeng.-Ze, C.; De-Chun, Z. Acta Phys. Chim. Sin. 2011, 27, 419.

(100) Zhang, J.; Yang, G.; Sun, Q.; Zheng, J.; Wang, P.; Zhu, Y.; Zhao, X. J. Renewable Sustainable Energy 2010, 2, 013104.

(101) Mieszawska, A. J.; Slawinski, G. W.; Zamborini, F. P. J. Am. Chem. Soc. 2006, 128, 5622.

(102) Hirose, F.; Kuribayashi, K.; Shikaku, M.; Narita, Y. Electrochem. Solid-State Lett. 2009, 12, B167.

(103) Wen, P.; Han, Y.; Zhao, W. Int. J. Photoenergy 2012.

(104) Turrion, M.; Macht, B.; Salvador, P.; Tributsch, H. I. Z. Phys. Chem. 1999, 212, 51.

(105) Hagfeldt, A.; Grätzel, M. Chem. Rev. 1995, 95, 49.

(106) Hagfeldt, A.; Grätzel, M. Acc. Chem. Res. 2000, 33, 269. 
(107) Gratzel, M. Photoelectrochemical cells. Nature 2001, 414, 338.

(108) Luitel, T.; Zamborini, F. P. Langmuir 2013, 29, 13582.

(109) He, H.; Gurung, A.; Si, L. Chem. Commun. 2012, 48, 5910.

(110) Luitel, T.; Druffel, T.; Zamborini, F.P. unpublished work.

(111) Quintana, M.; Edvinsson, T.; Hagfeldt, A.; Boschloo, G. J. Phys. Chem. C 2007, $111,1035$.

(112) Anderson, N. A.; Ai, X.; Lian, T. J. Phys. Chem. B 2003, 107, 14414.

(113) Bauer, C.; Boschloo, G.; Mukhtar, E.; Hagfeldt, A. J. Phys. Chem. B 2001, 105, 5585.

(114) Katoh, R.; Furube, A.; Yoshihara, T.; Hara, K.; Fujihashi, G.; Takano, S.; Murata, S.; Arakawa, H.; Tachiya, M. J. Phys. Chem. B 2004, 108, 4818.

(115) Szarko, J. M.; Neubauer, A.; Bartelt, A.; Socaciu-Siebert, L.; Birkner, F.; Schwarzburg, K.; Hannappel, T.; Eichberger, R. J. Phys. Chem. C 2008, 112, 10542.

(116) Willis, R. L.; Olson, C.; O'Regan, B.; Lutz, T.; Nelson, J.; Durrant, J. R. J. Phys. Chem. B 2002, 106, 7605.

(117) Ramakrishna, G.; Ghosh, H. N.; Singh, A. K.; Palit, D. K.; Mittal, J. P. J. Phys. Chem. B 2001, 105, 12786.

(118) Argazzi, R.; Bignozzi, C. A.; Heimer, T. A.; Castellano, F. N.; Meyer, G. J. J. Am. Chem. Soc. 1995, 117, 11815.

(119) Kuciauskas, D.; Freund, M. S.; Gray, H. B.; Winkler, J. R.; Lewis, N. S. J. Phys. Chem. B 2001, 105, 392.

(120) Pan, J.; Benko, G.; Xu, Y.; Pascher, T.; Sun, L.; Sundström, V.; Polivka, T. J. Am. Chem. Soc. 2002, 124, 13949.

(121) Asbury, J. B.; Hao, E.; Wang, Y.; Lian, T. J. Phy. Chem. B 2000, 104, 11957.

(122) Marcus, R. A. O. J. Chem. Phys. 1965, 43, 679.

(123) Gerischer, H.: In Physical Chemistry: an Advanced Treatise Academic Press: New York, 1970; Vol. IXA.

(124) Levich, V. G.: In Physical Chemistry: an Advanced Treatise; New York/London, 1970; Vol. IXB.

(125) Anderson, N. A.; Ai, X.; Chen, D.; Mohler, D. L.; Lian, T. J. Phys. Chem. B 2003, $107,14231$. 
(126) Bauer, C.; Teuscher, J.; Pelet, S.; Wenger, B.; Bonhote, P.; Nazeeruddin, M. K.; Zakeeruddin, S. M.; Comte, P.; Grätzel, M.; Moser, J.-E. Curr. Sci. 2010, 99, 343.

(127) Bauer, C.; Teuscher, J.; Brauer, J. C.; Punzi, A.; Marchioro, A.; Ghadiri, E.; De Jonghe, J.; Wielopolski, M.; Banerji, N.; Moser, J.-E. Chi. Int. J. Chem. 2011, 65, 704.

(128) Zeng, T.-W.; Liu, I.-S.; Huang, K.-T.; Liao, H.-C.; Chien, C.-T.; Wong, D. K.-P.; Chen, C.-W.; Wu, J.-J.; Chen, Y.-F.; Su, W.-F. J. Colloid Interface Sci. 2011, 358, 323.

(129) Zhang, F.; Srinivasan, M. P. Langmuir 2004, 20, 2309.

(130) Fattori, A.; Peter, L. M.; Belding, S. R.; Compton, R. G.; Marken, F. J. Electroanal. Chem. 2010, 640, 61.

(131) Wen, P.; Han, Y.; Zhao, W. Int. J. Photoenergy 2012, 2012, 906198.

(132) Tachibana, Y.; Moser, J. E.; Gratzel, M.; Klug, D. R.; Durrant, J. R. J. Phys. Chem. 1996, 100, 20056.

(133) Liang, Y.; Peng, B.; Chen, J. J. Phys. Chem. C 2010, 114, 10992.

(134) Dell'Orto, E.; Raimondo, L.; Sassella, A.; Abbotto, A. J. Mater. Chem. 2012, 22, 11364.

(135) Kallioinen, J.; Benkö, G.; Sundström, V.; Korppi-Tommola, J. E. I.; Yartsev, A. P. J. Phys. Chem. B 2002, 106, 4396.

(136) Benkö, G.; Kallioinen, J.; Korppi-Tommola, J. E.; Yartsev, A. P.; Sundström, V. J. Am. Chem. Soc. 2002, 124, 489.

(137) Benkö, G.; Kallioinen, J.; Myllyperkiö, P.; Trif, F.; Korppi-Tommola, J. E.; Yartsev, A. P.; Sundström, V. J. Phys. Chem. B 2004, 108, 2862.

(138) Bräm, O.; Cannizzo, A.; Phys. Chem. Chem. Phys. 2012, 14, 7934.

(139) Wenger, B.; Grätzel, M.; Moser, J.-E. J. Am. Chem. Soc. 2005, 127, 12150.

(140) Huber, R.; Sporlein, S.; Moser, J. E.; Gratzel, M.; Wachtveitl, J. T J. Phys. Chem. B 2000, 104, 8995.

(141) Valeur, B. Molecular Fluorescence: Principles and Applications, 2001.

(142) Smeigh, A. L.; Katz, J. E.; Brunschwig, B. S.; Lewis, N. S.; McCusker, J. K. J. Phys. Chem. C 2008, 112, 12065.

(143) Bauer, C.; Boschloo, G.; Mukhtar, E.; Hagfeldt, A. Int. J. Photoenergy 2002, 4, 17. 
(144) Huang, J.; Stockwell, D.; Boulesbaa, A.; Guo, J.; Lian, T. J. Phys. Chem. C 2008, 112,5203 .

(145) Tsubomura, H.; Matsumura, M.; Nomura, Y.; Amamiya, T. Nature 1976, 261, 402.

(146) Zhang, S.; Yang, X.; Qin, C.; Numata, Y.; Han, L. J. Mater. Chem. A 2014, 2, 5167.

(147) Li, J. Z.; Kong, F. T.; Wu, G. H.; Huang, Y.; Chen, W. C.; Dai, S. Y. Acta Phys. Chim. Sin. 2013, 29, 1851.

(148) Wang, P.; Zakeeruddin, S. M.; Moser, J. E.; Humphry-Baker, R.; Comte, P.; Aranyos, V.; Hagfeldt, A.; Nazeeruddin, M. K.; Gratzel, M. Adv Mater 2004, 16, 1806.

(149) Chiba, Y.; Islam, A.; Komiya, R.; Koide, N.; Han, L. Y. Appl. Phys. Lett. 2006, 88, 223505 .

(150) Bandaranayake, K. M. P.; Indika, S. M. K.; Prasad, W. P. M. G. M.; Tennakone, K. Coord. Chem. Rev. 2004, 248, 1277.

(151) Diamant, Y.; Chappel, S.; Chen, S. G.; Melamed, O.; Zaban, A. Coord. Chem. Rev. 2004, 248, 1271.

(152) Wang, S.; Yu, Z.; Zhang, X.; Ma, D. Optoelectron. Adv. Mater., Rapid Commun. 2013, 7, 549 .

(153) Ihara, M.; Tanaka, K.; Sakaki, K.; Honma, I.; Yamada, K J. Phys. Chem. B 1997, $101,5153-5157$.

(154) Choi, H.; Chen, W. T.; Kamat, P. V. ACS Nano 2012, 6, 4418.

(155) Xie, Y. A.; Huang, N.; Liu, Y. M.; Sun, W. W.; Mehnane, H. F.; You, S. J.; Wang, L. Y.; Liu, W.; Guo, S. S.; Zhao, X. Z. Electrochim. Acta 2013, 93, 202.

(156) Berglund, S. P.; Hoang, S.; Minter, R. L.; Fullon, R. R.; Mullins, C. B. J. Phys. Chem. C 2013, 117, 25248.

(157) Zalas, M. J. Rare Earths 2014, 32, 487.

(158) Huang, X. Y.; Han, S. Y.; Huang, W.; Liu, X. G. Chem. Soc. Rev. 2013, 42, 173. 201.

(159) Wang, G. F.; Peng, Q.; Li, Y. D. Acc. Chem. Res. 2011, 44, 322.

(160) Gomez, V.; Balu, A. M.; Serrano-Ruiz, J. C.; Irusta, S.; Dionysiou, D. D.; Luque, R.; Santamaría, J. Appl. Catal. A 2012, 441-442, 47. 
(161) Kralchevska, R.; Milanova, M.; Hristov, D.; Pintar, A.; Todorovsky, D. Mater. Res. Bull. 2012, 47, 2165.

(162) Fernando, K.; Pandit, B.; Liu, J.; Alphenaar, B. W. Chem. Phys. Lett. 2014, 592, 155.

(163) Hafez, H.; Saif, M.; Abdel-Mottaleb, M. S. A. J. Power Sources 2011, 196, 5792.

(164) Wang, S.; Yu, Z. C.; Zhang, X. J.; Ma, D. Optoelectron. Adv. Mater., Rapid Commun. 2013, 7, 549.

(165) Wu, J.; Xie, G.; Lin, J.; Lan, Z.; Huang, M.; Huang, Y. J. Power Sources 2010, 195 , 6937.

(166) Yao, Q. H.; Liu, J. F.; Peng, Q.; Wang, X.; Li, Y. D. Chem-Asian J. 2006, 1, 737.

(167) Zalas, M.; Klein, M. Int. J. Photoenergy 2012, 2012, 1.

(168) Zhang, J.; Peng, W.; Chen, Z.; Chen, H.; Han, L. J. Phys. Chem. C 2012, 116, 19182.

(169) Zhang, J.; Shen, H.; Guo, W.; Wang, S.; Zhu, C.; Xue, F.; Hou, J.; Su, H.; Yuan, Z. J. Power Sources 2013, 226, 47.

(170) Chang, W.-C.; Lee, C.-H.; Yu, W.-C.; Lin, C.-M. Nanoscale Res. Lett. 2012, 7, 1.

(171) Sibu, C. P.; Kumar, S. R.; Mukundan, P.; Warrier, K. G. K. Chem. Mater. 2002, 14, 2876.

(172) Chang, J.; Ning, Y. H.; Wu, S. L.; Niu, W. B.; Zhang, S. F. Adv. Funct. Mater. 2013, 23,5910 .

(173) Liang, L. L.; Liu, Y. M.; Bu, C. H.; Guo, K. M.; Sun, W. W.; Huang, N.; Peng, T.; Sebo, B.; Pan, M. M.; Liu, W.; Guo, S. S.; Zhao, X. Z. Adv Mater 2013, 25, 2174.

(174) Lim, M. J.; Ko, Y. N.; Kang, Y. C.; Jung, K. Y. RSC Adv. 2014, 4, 10039.

(175) Ramasamy, P.; Kim, J. Chem. Commun. 2014, 50, 879.

(176) Shan, G. B.; Demopoulos, G. P. Adv Mater 2010, 22, 4373.

(177) Wang, J. L.; Wu, J. H.; Lin, J. M.; Huang, M. L.; Huang, Y. F.; Lan, Z.; Xiao, Y. M.; Yue, G. T.; Yin, S.; Sato, T. Chem. Sus. Chem. 2012, 5, 1307.

(178) Yu, J.; Yang, Y. L.; Fan, R. Q.; Liu, D. Q.; Wei, L. G.; Chen, S.; Li, L.; Yang, B.; Cao, W. W. Inorg. Chem. 2014, 53, 8045.

(179) Hafez, H.; Wu, J. H.; Lan, Z.; Li, Q. H.; Xie, G. X.; Lin, J. M.; Huang, M. L.; Huang, Y. F.; Abdel-Mottaleb, M. S. Nanotechnology 2010, 21, 6.

(180) Chen, S. Y.; Lin, J. M.; Wu, J. H. J. Mater. Sci.-Mater. Electron. 2014, 25, 2060

(181) He, W.; Atabaev, T. S.; Kim, H. K.; Hwang, Y. HJ Phys Chem C 2013, 117, 17894. 
(182) Li, Q. B.; Lin, J. M.; Wu, J. H.; Lan, Z.; Wang, Y.; Peng, F. G.; Huang, M. L. J. Lumines. 2013, 134, 59.

(183) Yao, N. N.; Huang, J. Z.; Fu, K.; Liu, S. Y.; Dong, E.; Wang, Y. H.; Xu, X. J.; Zhu, M.; Cao, B. Q. J. Power Sources 2014, 267, 405.

(184) Zhu, G.; Wang, X. J.; Li, H. L.; Pan, L. K.; Sun, H. C.; Liu, X. J.; Lv, T.; Sun, Z. Chem. Commun. 2012, 48, 958.

(185) Li, Y.; Pan, K.; Wang, G. F.; Jiang, B. J.; Tian, C. G.; Zhou, W.; Qu, Y.; Liu, S.; Feng, L.; Fu, H. G. Dalton Trans. 2013, 42, 7971.

(186) Han, C. H.; Lee, H. S.; Lee, K. W.; Han, S. D.; Singh, I. Bull. Korean Chem. Soc. 2009, 30, 219.

(187) Wang, J. L.; Lin, J. M.; Wu, J. H.; Huang, M. L.; Lan, Z.; Chen, Y.; Tang, S.; Fan, L. Q.; Huang, Y. F. Electrochim. Acta 2012, 70, 131.

(188) Huang, J. H.; Hung, P. Y.; Hu, S. F.; Liu, R. S. J. Mater. Chem. 2010, 20, 6505.

(189) Yu, H.; Xue, B. F.; Liu, P. R.; Qiu, J. X.; Wen, W.; Zhang, S. Q.; Zhao, H. J. ACS Appl. Mater. Interfaces 2012, 4, 1289.

(190) Qin, P.; Domanski, A. L.; Chandiran, A. K.; Berger, R.; Butt, H. J.; Dar, M. I.; Moehl, T.; Tetreault, N.; Gao, P.; Ahmad, S.; Nazeeruddin, M. K.; Gratzel, M. Nanoscale 2014, 6, 1508.

(191) Bazzi, R.; Brenier, A.; Perriat, P.; Tillement, O. J. Lumin. 2005, 113, 161.

(192) Lin, C. J.; Yu, W. Y.; Chien, S. H. Appl. Phys. Lett. 2007, 91, 2331201.

(193) Tu, W. K.; Lin, C. J.; Chatterjee, A.; Shiau, G. H.; Chien, S. H. J. Power Sources 2012, 203, 297.

(194) Zhang, H. M.; Yu, H.; Han, Y. H.; Liu, P. R.; Zhang, S. Q.; Wang, P.; Cheng, Y. B.; Zhao, H. J. Nano Res. 2011, 4, 938.

(195) Han, L.; Koide, N.; Chiba, Y.; Mitate, T. Appl. Phys. Lett. 2004, 84, 2433.

(196) Adachi, M.; Sakamoto, M.; Jiu, J.; Ogata, Y.; Isoda, S. J. Phys. Chem. B 2006, 110, 13872.

(197) Dieke, G. H.: Spectra and Energy Levels of Rare Earth Ions in Crystals; John Wiley \& Sons Inc.: New York, NY, 1968.

(198) Greiner, M. T.; Chai, L.; Helander, M. G.; Tang, W.-M.; Lu, Z.-H. Adv. Funct. Mater. 2012, 22, 4557. 
(199) Petit, L.; Svane, A.; Szotek, Z.; Temmerman, W. M. Phys. Rev. B 2005, 72, 2051181.

(200) Zhang, J.; Hughes, T. F.; Steigerwald, M.; Brus, L.; Friesner, R. A J. Am. Chem. Soc. 2012, 134, 12028.

(201) Pandit, B.; Luitel, T.; Cummins, D. R.; Thapa, A. K.; Druffel, T.; Zamborini, F.; Liu, J. J. Phys. Chem. A 2013, 117, 13513.

(202) Hamann, T. W.; Jensen, R. A.; Martinson, A. B. F.; Van Ryswyk, H.; Hupp, J. T. Energy Envi. Sci. 2008, 1, 66.

(203) Nazeeruddin, M. K.; Baranoff, E.; Grätzel, M. Sol. Energy 2011, 85, 1172.

(204) Sue Ferrere, B. A. G. Proc. - Electrochem. Soc. 2001, 10, 161.

(205) Murakoshi, K.; Kano, G.; Wada, Y.; Yanagida, S.; Miyazaki, H.; Matsumoto, M.; Murasawa, S. J. Electroanal. Chem. 1995, 396, 27.

(206) Wu, K.-J.; Shen, K.; Yu, Y.; Wang, D. Chin. J. Chem. Phys. 2012, 25, 733.

(207) Wu, M.; Yang, Z. H.; Jiang, Y. H.; Zhang, J. J.; Liu, S. Q.; Sun, Y. M. J. Solid State Electrochem. 2010, 14, 857.

(208) Yum, J.-H.; Nakade, S.; Kim, D.-Y.; Yanagida, S. I J. Phys. Chem. B 2006, 110, 3215 .

(209) Jun, H. K.; Careem, M. A.; Arof, A. K. Renewable Sustainable Energy Rev. 2013, 22,148 .

(210) Tvrdy, K.; Frantsuzov, P. A.; Kamat, P. V. Proc. Natl. Acad. Sci. U. S. A. 2011, 108, 29.

(211) Enomoto, M.; Taniguchi, K.; Ihara, M. ECS Trans. 2010, 25, 37.

(212) Martinson, A. B. F.; Elam, J. W.; Liu, J.; Pellin, M. J.; Marks, T. J.; Hupp, J. T. Nano Lett. 2008, 8, 2862.

(213) Chen, P.-Y.; Dang, X.; Klug, M. T.; Qi, J.; Dorval Courchesne, N.-M.; Burpo, F. J.; Fang, N.; Hammond, P. T.; Belcher, A. M. ACS Nano 2013, 7, 6563.

(214) Zhang, Q. F.; Cao, G. Z. Nano Today 2011, 6, 91.

(215) Mor, G. K.; Shankar, K.; Paulose, M.; Varghese, O. K.; Grimes, C. A. Nano Lett. 2005, 6, 215.

(216) Jimenez-Cadena, G.; Comini, E.; Ferroni, M.; Vomiero, A.; Sberveglieri, G. Mater. Chem. Phys. 2010, 124, 694.

(217) Su, Y.-H.; Ke, Y.-F.; Cai, S.-L.; Yao, Q.-Y. Light: Sci. Appl. 2012, 1, e14/11. 
(218) Brown, M. D.; Suteewong, T.; Kumar, R. S. S.; D'Innocenzo, V.; Petrozza, A.; Lee, M.; Wiesner, U.; Snaith, H. J. Nano Lett. 2011, 11, 438.

(219) Ihara, M.; Tanaka, K.; Sakaki, K.; Honma, I.; Yamada, K. J. Phys. Chem. B 1997, $101,5153$.

(220) Furube, A.; Du, L.; Hara, K.; Katoh, R.; Tachiya, M. J. Am. Chem. Soc. 2007, 129, 4852.

(221) Gratzel, M. Nature 2003, 421, 586.

(222) Subramanian, V.; Wolf, E. E.; Kamat, P. V. J. Am. Chem. Soc. 2004, 126, 4943.

(223) Standridge, S. D.; Schatz, G. C.; Hupp, J. T. Langmuir 2009, 25, 2596.

(224) Wen, P.; Xue, M.; Ishikawa, Y.; Itoh, H.; Feng, Q. ACS Appl. Mater. Interfaces 2012, $4,1928$.

(225) Baba, A.; Wakatsuki, K.; Shinbo, K.; Kato, K.; Kaneko, F. J. Mater. Chem. 2011, $21,16436$.

(226) Ding, I. K.; Zhu, J.; Cai, W.; Moon, S.-J.; Cai, N.; Wang, P.; Zakeeruddin, S. M.; Grätzel, M.; Brongersma, M. L.; Cui, Y.; McGehee, M. D. Adv. Energy Mater. 2011, $1,52$.

(227) Gangishetty, M. K.; Lee, K. E.; Scott, R. W. J.; Kelly, T. L. Acs Appl.Mater.Interfaces 2013, 5, 11044.

(228) Wang, Q.; Butburee, T.; Wu, X.; Chen, H.; Liu, G.; Wang, L. J. Mater. Chem. A 2013, $1,13524$.

(229) Standridge, S. D.; Schatz, G. C.; Hupp, J. T. J. Am. Chem. Soc. 2009, 131, 8407.

(230) Zedler, L.; Theil, F.; Csaki, A.; Fritzsche, W.; Rau, S.; Schmitt, M.; Popp, J.; Dietzek, B. RSC Adv. 2012, 2, 4463.

(231) Jana, N. R.; Gearheart, L.; Murphy, C. J. J. Phys. Chem. B 2001, 105, 4065.

(232) Lee, K. E.; Gomez, M. A.; Elouatik, S.; Demopoulos, G. P. Langmuir 2010, 26, 9575 .

(233) Jeong, N. C.; Prasittichai, C.; Hupp, J. T. Langmuir 2011, 27, 14609.

(234) Bram, O.; Cannizzo, A.; Chergui, M. Phys. Chem. Chem. Phys. 2012, 14, 7934.

(235) Wang, Q.; Ito, S.; Grätzel, M.; Fabregat-Santiago, F.; Mora-Sero, I.; Bisquert, J.; Bessho, T.; Imai, H. J. Phys. Chem. B 2006, 110, 25210. 


\section{APPENDIX-1 LIST OF ABBREVIATIONS}

$\begin{array}{ll}\text { AC } & =\text { Alternating current } \\ \text { ACN } & =\text { Acetonitrile } \\ \text { AES } & =\text { Auger electron spectroscopy } \\ \text { AFM } & =\text { Atomic Force Microscopy } \\ \text { APhS } & =\text { p-Aminophenyltrimethoxysilane } \\ \text { APTES } & =3 \text {-Aminopropyltriethoxysilane } \\ \text { ATR-FTIR } & =\text { Attenuated total reflectance Fourier transform infrared spectroscopy } \\ \text { Au NPs } & =\text { Gold nanoparticles } \\ \text { BA } & =\text { Benzoic acid } \\ \text { CB } & =\text { Conduction band } \\ \text { CE } & =\text { Counter electrode } \\ \text { CN } & =\text { Carbon-nitrogen } \\ \text { DCC } & =\text { 'N-Dicyclohexylcarbodiimide } \\ \text { DCM } & =\text { Dichloromethane } \\ \text { DMAP } & =4-\text { Dimethylaminopyridine } \\ \text { DRS } & =\text { Diffuse reflectance spectroscopy } \\ \text { DSSC } & =\text { Dye-sensitized solar cell } \\ \text { EIS } & =\text { Electrochemical impedance spectroscopy } \\ \text { ESA } & =\text { Excited-state absorption } \\ \text { ET } & =\text { Electron transfer } \\ \text { FTO } & =\text { Fluorine doped tin-oxide } \\ \text { GSB } & =\text { Ground state bleaching } \\ \text { HOMO } & =\text { Highest occupied molecular orbital } \\ \text { IPCE } & =\text { Incident photon-to-current-conversion efficiency } \\ \text { ITO } & =\text { Indium tin-oxide } \\ \text { LUMO } & =\text { Lowest unoccupied molecular orbital } \\ \text { NCS } & =\text { Thiocyanate } \\ \text { NPs } & =\text { Nanoparticles } \\ \text { OD } & =\text { Optical Density } \\ \text { PA } & =\text { Photoanode } \\ \text { PCE } & =\text { Power conversion efficiency }\end{array}$




$\begin{array}{ll}\text { Pt } & =\text { Platinum } \\ \text { SEM } & =\text { Scanning electron microscopy } \\ \text { TAAPS } & =\text { Transient absorption pump-probe spectroscopy } \\ \text { UTAS } & =\text { Ultrafast transient absorption spectroscopy } \\ \text { UV-vis } & =\text { Ultraviolet visible spectroscopy }\end{array}$




\section{APPENDIX-2 COPYRIGHT PERMISSION}

Reference \# 108 Reprint with the permission from

Luitel, T.; Zamborini, F. P. Langmuir 2013, 29, 13582.

Reference \# 201

Reprint with the permission from

Pandit, B.; Luitel, T.; Cummins, D. R.; Thapa, A. K.; Druffel, T.; Zamborini, F.; Liu, J. J.Phys. Chem. A 2013, 117, 13513-13523. 


\section{CURRICULUM VITAE}

\section{Tulashi Luitel}

Department of Chemistry

t0luit01@louisville.edu

Phone: (502) 852-5982 (Office)

University of Louisville

Phone: (502) 387-0258 (Mobile)

2320 South Brook Street

Louisville, KY 4029

\section{Professional Objective}

To use experienced knowledge and skills developed in the field of photovoltaics, nanostructured materials synthesis, electrochemistry, and surface chemistry for productive academic/research careers.

\section{Essential skills and Qualifications}

Ph.D. in Chemistry

2008-2015

Department of Chemistry

University of Louisville, KY

M.S. in Chemistry

2008-2012

Department of Chemistry

University of Louisville, KY

M.S. in Physics

Department of Chemistry

2006-2008

University of Louisville, KY 


\section{Research Experience}

Fabrication skills in photovoltaic devices, instrumentation and measurement system for solar devices, designing nanomaterials for various purposes and study their photophysics and photochemistry mainly through charge transfer dynamics at nanoscale.

\section{Characterization Techniques}

UV-vis, ATR-FTIR, Electrochemistry, EIS, SEM, AFM, Raman, XPS, SECM.

Electrochemical impedance spectroscopy (EIS).

Ultrafast transient absorption spectroscopy (UTAS).

Optical microscopy.

Teaching Experience (Tribhuvan University)

1993-2006

Thermodynamics, Modern Physics, Advanced Level Physics, Physical Electronics.

Teaching Experience (University of Louisville)

2006-2015

Undergraduate laboratory classes of Chemistry and Physics.

\section{Community Services}

Vice-president - Nepalese Student Association, University of Louisville

2012-2013

Judge - Louisville Regional Science \& Engineering Fair

2007-2014

Observer - Office of Examination Controller, Tribhuvan University

1997-2006

Instructor - Design and conduct scientific experiments for High school science teachers

Representative - University Teachers Association (Tribhuvan University)

2002-2004 


\section{Research Fund and Award}

Conn Center for Renewable Energy Research, University of Louisville. Nanoscale Materials Research Fund (under DOE project).

2009-2013

Mahendra Morang Adarsha Multiple Campus (Tribhuvan University), Selected for College Scholarship Award.

$1986-1988$

University Grants Commissions (UGC), Nepal.

2000-2004

Science Promotion Grants (Nepal Academy of Science and Technology), Nepal.

2002

\section{Publications}

- "Increased efficiency of dye-sensitized solar cells using a rare earth oxide/titania composite acceptor" Luitel, T.; Fernando, K.; Tatum, B. S.; Alphenaar, B. W.; Zamborini, F.P., submitted to J. of Power Sources.

- "Synthesis of mesoporous birnessite- $\mathrm{MnO}_{2}$ composite as a cathode electrode for lithium battery" Thapa, A.K.; Pandit, B.; Thapa, R.; Luitel, T.; Paudel, H.S.; Sumanasekera, G.; Sunkara, M.K.; Gunawardhana, N.G.; Ishihara, T.; Yoshio, M. Electrochimica Acta ,2014, 116,188- 193.

- "Spectroscopic Investigation of Photoinduced Charge-Transfer Processes in

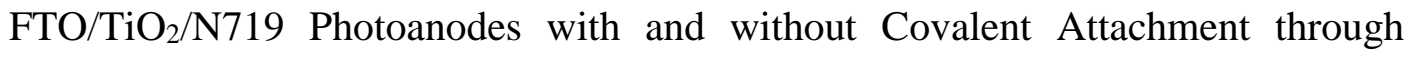
Silane-Based Linkers" Pandit, B.; Luitel, T.; Cummins, D.R.; Thapa, A.K.; Druffel, T.; Zamborini, F.P.; Liu, J. J. Phys. Chem. A, 2013, 117, 13513-13523.

- "Covalent Modification of Photoanodes for Stable Dye-Sensitized Solar Cells", Luitel, T.; Zamborini, F.P. Langmuir, 2013, 29, 13582-13594.

- "Determination of Maximum Water Holding Capacity of Soils of Different Parts of Kathmandu Valley by Gamma Ray-attenuation Technique", Luitel, T.; Kafle, S.R. Journal of Nepal Physical Society, 13, 1996, 34-40. 


\section{Conference Presentations}

- "Towards the Maximization of Dye-sensitized Solar Cells Using Rare-earth Metal Oxides and Metal Nanoparticles", Luitel, T.; Fernando, K.; Alphenaar, B.W.; Zamborini, F.P. Southeastern Regional Meeting American Chemical Society (SERMACS), October 16-19, 2014, Nashville, Tennessee.

- "The Use of Gold Nanoparticles in Dye-Sensitized Solar Cells and Biosensing", Zamborini, F. P.; Luitel, T.; Beeram, S.; Bao, L.; Fang, A. Southeastern Regional Meeting American Chemical Society (SERMACS), November 12-16, 2013, Atlanta, Georgia.

- "Interfacial Surface Modification of Photoanodes at Molecular level for hermetically Stable Dye-sensitized Solar Cells", Luitel, T.; Zamborini, F.P.; American Chemical Society Meeting \& Exposition, September 8-12, 2013, Indianapolis, Indiana.

- "Spectroscopic Investigation of Photoinduced Charge-Transfer Processes in FTO/TiO $/$ /N719 Photoanodes with and without Covalent Attachment through Silane-Based Linkers", Pandit, B.; Luitel, T.; Cummins, D.R.; Thapa, A.K.; Druffel, T.; Zamborini, F.P.; Liu, J. National American Chemical Society Meeting \& Exposition, September 8-12, 2013, Indianapolis, Indiana.

- "Interfacial Surface Modification of Photoanodes at Molecular Level for Chemically Stable Dye-Sensitized Solar Cells", Luitel, T.; Zamborini, F.P. Kentucky Statewide Workshop: Renewable Energy and Energy Efficiency, March 24-26, 2013, Louisville, Kentucky.

- "Interfacial Surface Modification of Photoanodes at Molecular Level for Chemically Stable Dye-Sensitized Solar Cells", Luitel, T.; Zamborini, F.P. Graduate Student Symposium, University of Louisville, March 24, 2013, Louisville, Kentucky.

- "Interfacial Nano-scale Modification of Photoanodes for the Optimization of Parameters of Dye-sensitized Solar Cells", Luitel, T.; Zamborini, F.P. SERMACS, November 14-17, 2012, Raleigh, North Carolina.

- "Interfacial Surface Modification of Photoanodes by Nanolinkers and Metal Nanoparticles for Chemically Stable and Highly Efficiency Dye-sensitized Solar Cells", Luitel, T.; Zamborini, F.P. Graduate Student Symposium, March 24, 2012, University of Louisville, Kentucky. 
- "Chemical Modification of Photoanode for Stable Dye-sensitized Solar Cells", Luitel, T.; Zamborini, F.P. Pittcon Conference and Exposition, March 11-15, 2012, Orlando, Florida.

- "Chemical Modification of Photoanode for Stable Dye-sensitized Solar Cells", Luitel, T.; Zamborini, F.P. SERMACS, October 26-29, 2011, Richmond, Virginia.

- "Improved Photovoltaic Performance of Dye-sensitized Solar Cells by Using 3Aminopropyltriethoxysilane and Gold Nanoparticles", Luitel, T.; Zamborini, F.P. National ACS Meeting, August 28-September 1, 2011, Denver, Colorado.

- "Improved Photovoltaic Performance of Dye-sensitized Solar Cells by Using 3Aminopropyltriethoxysilane and Gold Nanoparticles", Luitel, T.; Zamborini, F.P. CERMAS, June 8-10, 2011, Indianapolis, Indiana.

- "Chemical Modification of Photoanode Used in Dye-sensitized Solar Cells", Luitel, T.; Zamborini, F.P. Kentucky Statewide Workshop: Renewable Energy \& Energy Efficiency, March 13-15, 2011, Louisville, Kentucky.

- "Monitoring of X-radiation in Private and Public Sectors in Biratnagar Submetropolitan City", Luitel, T.; Aryal, S.K. Radiological Protection of Patient in Use of Ionizing Radiation, March 22-23, 2002, Sanjaya Gandhi Postgraduate Institute of Medical Sciences, Luckow, India.

- "Modern Techniques in NMR Spectroscopy-2D NMR", Central Department of Chemistry, December 13-14, 1995, Kathmandu, Nepal.

\section{Other Presentation}

- "Chemical Synthesis and Characterization of Gold Nanoparticles Used in the Detection of Cancer Cells", Luitel, S. Louisville Science and Engineering Fair, Louisville, Kentucky, March 8, 2013. (Award First, Biochemistry). She synthesized different sizes of gold nano-particles, connects to $\mathrm{TiO}_{2}$ nanoparticles, and made atomic force microscopy images. Gold nanoparticles are being used in detection of cancer cells.

- "Power of Nanotechnology", Shreeyanka Luitel, International Sustainable World Energy Engineering Environmental Project Olympiad (I-SWEEP), May 6-11, 2013 (Award Honor, Engineering). She overall displayed all her the aforementioned work with some demonstrations of nano-technology. 
- "Effective Calculation of Cost of Laboratory Preparation of Dye-sensitized Solar Cells Using Electrolysis of Water as a Model”, Luitel, B. Louisville Science and Engineering Fair, Louisville, Kentucky, March 7, 2015 (Award First, Chemistry).

- "Effective Calculation of Cost of Laboratory Preparation of Dye-sensitized Solar Cells Using Electrolysis of Water to Produce $\mathrm{H}_{2}$ gas, Luitel, B. Kentucky Science \& Engineering Fair, Richmond, Kentucky, March 28, 2015 (Award First, Chemistry).

\section{Professional Associations}

- American Chemical Society (ACS)

2008 - Present

- American Chemical Society's Analytical Division

2010 - Present

- Nepal Physical Society (NPS),

Life-Member

- Tribhuvan University Teachers association

1997 - 2014

\section{Software for Data analysis}

Microsoft word, Microsoft Excel, Origin, Igor, Matlab.

\section{Language Skills}

Nepali (Native), English (2 ${ }^{\text {nd }}$ language), Sanskrit (Childhood language), Hindi (Learned language)

\section{References}

\section{Dr. Francis P. Zamborini (Ph.D. Advisor)}

Professor

Department of Chemistry

University of Louisville

2320 South Brook Street

Louisville, KY 40292, USA

Phone: (502) 8526550

f.zamborini@louisville.edu

http://stage.louisville.edu/faculty/fpzamb01/home.html 


\author{
Dr. Bruce W. Alphenaar \\ Professor and ECE Chair \\ Department of Electrical and Computer Engineering \\ University of Louisville \\ J.B. Speed School of Engineering - W. S. Speed \\ Louisville, KY 40292, USA \\ Phone: (502) 8521554 \\ Email: bwalph01@louisville.edu
}

\title{
Dr. Jinjun Liu
}

Assistant Professor

Department of Chemistry

University of Louisville

2320 South Brook Street

Louisville, KY 40292, USA

Phone: 502-852-1223

Email j.liu@ @ouisville.edu 